\title{
THE EFFECTS OF TEMPORALITY AND SPATIALITY DUE TO AGED DATA IN LIFE CYCLE ASSESSMENTS
}

by

\section{Jonathan Pryshlakivsky}

MASc, Ryerson University, Toronto, 2009

BASc, Ryerson University, Toronto, 2006

BA, McMaster University, Hamilton, 1998

BEng, McMaster University, Hamilton, 1996

\author{
A dissertation \\ presented to Ryerson University \\ in partial fulfillment of the \\ requirements for the degree of \\ Doctor of Philosophy \\ in the Program of \\ Environmental Applied Science and Management
}

Toronto, Ontario, Canada, 2015

() Jonathan Pryshlakivsky 2015 
AUTHOR'S DECLARATION

I hereby declare that I am the sole author of this dissertation. This is a true copy of the dissertation, including any required final revisions, as accepted by my examiners.

I authorize Ryerson University to lend this dissertation to other institutions or individuals for the purpose of scholarly research.

I further authorize Ryerson University to reproduce this dissertation by photocopying or by other means, in total or in part, at the request of other institutions or individuals for the purpose of scholarly research.

I understand that my dissertation may be made electronically available to the public. 
Title: The Effects of Temporality and Spatiality Due to Aged Data in Life Cycle Assessments

Degree: Doctor of Philosophy, 2015, Jonathan Pryshlakivsky

Environmental Applied Science and Management Program, Ryerson University

\begin{abstract}
Life cycle assessment is a relatively new_although decades old — method for assessing the environmental impacts of goods and services. It seeks to quantify these impacts in such a manner as to facilitate informed decisions regarding different, yet equally viable, options. However, this aim must be conditional on the notion that these impacts are measured with a number of associated qualifications or caveats, two of which is subject of this work. As subject matter, temporality and spatiality in life cycle assessment are both very broad, although this dissertation focuses specifically on temporality and spatiality due to age of data. The structure of the dissertation follows three distinct phases. The first phase contextualized the subject matter and its relation towards standardization of life cycle assessment methods. In doing so, it identifies and contextualizes the subject matter. The second phase identified Greenhouse gases, Regulatory Emissions, and Energy use in Transportation 2 as an ideal model on which to assess temporality and spatiality due to age of data since it models the life cycle assessment of an assortment of different vehicles. This phase also involved data collection, and uses a platform of assessment tools including Monte Carlo simulations, analysis of variance, $\mathrm{F}$ tests, regression analysis, and tests for non-normality (kurtosis and skewness). Building on the second phase, the third phase moved beyond the original phases by more than doubling the amount of materials of manufacture to be studied and adding further tools for assessment, the mainstay of which are regression analyses. Overall, this study found that the use of Monte Carlo simulations and analysis of variance are useful for identifying long term variation in energy intensity of materials. F-tests were useful in identifying which materials showed effects owing to spatiality. Although not in all instances, tests for non-normality identified which circumstances merit log transformation to bring about more accurate results. Linear regression techniques were used as a posterior test to confirm the origins of the variation seen in the Monte Carlo simulations and the
\end{abstract}


analysis of variation. Moving ahead, this study pointed to the need for more concerted efforts in data promulgation. 


\section{ACKNOWLEDGEMENTS}

I wish to thank Dr. Cory Searcy for his kind, thoughtful, and enthusiastic navigation throughout this process.

I would also like to thank Dr. Andrew Laursen for introducing me to the potential benefits of Monte Carlo simulations and further assistance with their statistical analysis.

I would also like to thank the department of Chemistry and Biology for kindly giving me the opportunity to work and gain teaching experience: Drs. Lynda McCarthy, Andrew Laursen, Kim Gilbride, Martina Hausner, Noel George, Vadim Bostan, Dérick Rousseau, Andrew McWilliams, Monica Sauer and Charlotte de Araujo, as well as staff members Karen Puddephatt, Liberty Victorio-Walz, Mariam de Jong, and Stephanie Grouios all of whom made my life easier. 


\section{DEDICATION}

This work is dedicated to my mother Alexandra Yaroslava without whom this would not have been possible. 


\section{Table of Contents}

Page

Author's Declaration

ii

Abstract

iii

Acknowledgements

Dedication

V

Table of Contents

vi

List of Abbreviations

vii

List of Tables

$\mathrm{X}$

List of Figures

xiii

xvi

CHAPTER 1. Introduction.

1.1 Life Cycle Assessment: An Outline 1

1.2 Sources and Sinks 4

1.3 Uncertainty and Variance $\quad 7$

1.4 Problem Identification 12

1.5 Research Objectives and Methodological Approach 13

$\begin{array}{ll}\text { 1.5.1 Model Choice } & 13\end{array}$

1.5.2 Data Collection 16

1.5.3 Monte Carlo Simulations 19

1.5.4 Analysis of Variation and F tests 21

$\begin{array}{ll}\text { 1.5.5 Regression Analysis } & 22\end{array}$

1.5.6 Tests for Non-normal Data 23

1.6 Glossary of Key Definitions 23

1.7 Structure of the Dissertation 28

CHAPTER 2. Life Cycle Assessment and the progress towards standardization 30 and normalization.

2.1 Overview of LCA Development and Standardization 30

$\begin{array}{ll}2.2 \text { Pre-2006 } & 31\end{array}$ 
CHAPTER 3. Analysis of how energy intensities from vehicle manufacturing materials vary with the age of data and the region they represent.

3.1 Introduction $\quad 44$

3.2 Methods 49

3.2.1 Monte Carlo Simulations $\quad 50$

3.2.2 Data collection and utilization $\quad 55$

3.2.3 Coefficient of variance (CV), standard error (SE), and ANOVA 61

3.2.4 Skewness and Kurtosis 63

3.2.5 Limitations $\quad 63$

3.3 Results and Discussion $\quad 64$

3.3.1 Monte Carlo simulations and analysis of variance (temporality) 64

3.3.2 Discussion related to the use of intervals for Monte Carlo 72

Simulations

3.3.3 Discussion on the possibility of non-normality in the temporality 76 Results

3.3.4 Monte Carlo simulations and analysis of variance (spatiality) 76

$\begin{array}{ll}3.4 \text { Conclusions } & 78\end{array}$

CHAPTER 4. Monte Carlo simulations and regression analysis for assessing $\quad 82$ temporality due to age of data.

4.1 Introduction $\quad 82$

$\begin{array}{ll}\text { 4.2 Methods } & 86\end{array}$

4.2.1 Data collection $\quad 86$

4.2.2 Monte Carlo simulations and analysis of variation 87

$\begin{array}{ll}4.2 .3 \text { Regression analysis } & 90\end{array}$

4.2.4 Skewness and kurtosis $\quad 92$ 
$\begin{array}{ll}\text { 4.2.5 Limitations } & 93\end{array}$

4.3 Results and Discussion 94

4.3.1 Monte Carlo simulations and analysis of variance 94

$\begin{array}{ll}4.3 .2 \text { Regression analysis } & 109\end{array}$

$\begin{array}{ll}\text { 4.3.3 Skewness and kurtosis } & 110\end{array}$

$\begin{array}{ll}\text { 4.3.4 Caveat regarding data availability } & 112\end{array}$

$\begin{array}{ll}4.4 \text { Conclusions } & 114\end{array}$

$\begin{array}{ll}\text { CHAPTER 5. Conclusions. } & 115\end{array}$

5.1 General Conclusions 115

$\begin{array}{ll}\text { 5.1.1 Contributions } & 116\end{array}$

$\begin{array}{ll}\text { 5.1.2 Implications } & 119\end{array}$

5.2 Limitations of This Study 120

5.3 Future Work 122

Appendix A. References for Body of Dissertation 126

Appendix B. References for Data Collection 143

Appendix C. Complete List of Countries in Figures 3.12 and 3.13 163

$\begin{array}{lr}\text { REFERENCES } & 164\end{array}$ 


\section{LIST OF ABBREVIATIONS}

ABS Acrylonitril Butadiene Styrene

ANOVA Analysis of Variance

BOE Barrels of Oil Equivalents

CFRP Carbon Fibre Reinforced Plastic

CIEEDAC The Canadian Industrial Energy End-Use Data and Analysis Centre

CIRAIG Interuniversity Research Centre for the Life Cycle of Products, Processes and Services (Canada)

CSR Corporate Social Responsibility

CV Coefficient of Variance

EA Environmental Assessment

EIO-LCA Economic Input-Output—Life Cycle Assessment

EOL End-of-life

EPA US Environmental Protection Agency

EPDM Ethylene Propylene Diene Monomer

EV Electric Vehicle

FCV Fuel Cell Vehicle

GFRP Glass Fibre Reinforced Plastic

GHG Green House Gas

GPPS General Purpose Polystyrene

GREET Greenhouse gases, Regulated Emissions, and Energy use in Transportation (Argonne National Laboratory)

GWP Global Warming Potential

HDPE High-Density Polyethylene 


\begin{tabular}{ll} 
HIPS & High Impact Polystyrene \\
HEV & Hybrid Engine Vehicle \\
ICE & Inventory of Carbon and Energy (University of Bath, UK) \\
ICEV & Internal Combustion Engine Vehicle \\
ISO & International Organization for Standardization \\
kWh & Kilowatt hours \\
LAR & Least Absolute Residuals (regression) \\
LCA & Life Cycle Assessment \\
LCI & Life Cycle Inventory \\
LCIA & Life Cycle Impact Assessment \\
LDPE & Low-Density Polyethylene \\
LLDPE & Linear Low-Density Polyethylene \\
LS & Least Squares (regression) \\
PC & Polycarbonate \\
PET & Polyethylene Terephthalate \\
PFSA & Perfluorosulfonic Acid \\
PHEV & Plug-in Hybrid Engine Vehicle \\
POCP & Photochemical Ozone Creation Potentials \\
PNRG & Pseudo-random Number Generator \\
PTFE & Polytetrafluoroethylene \\
PUR(F) & Flexible Polyurethane \\
PUR(R) & Rigid Polyurethane \\
PUT & Pick-up Truck \\
\hline
\end{tabular}


PP Polypropylene

PVC Polyvinyl Chloride

SD Standard Deviation

SPINE Sustainable Product Information Network for the Environment (Sweden)

SUV Sport Utility Vehicle 


\section{LIST OF TABLES}

Page

\section{CHAPTER 1}

Table $1.1 \quad$ Types of Uncertainty (by study author). 9

Table 1.2 Details related to the simulation phases of the dissertation. 15

Table 1.3 Principle Classifications of Life Cycle Inventory Data. 18

Table 1.4 Breakdown of Types of Documents Used in Phase 3 of the Dissertation. 18

\section{CHAPTER 2}

Table 2.1 Summation of pre-2006 critiques of the ISO 14040 series. 34

Table 2.2 Summation of Recommendations Since 2006 and Their Potential 37

Viability.

\section{CHAPTER 3}

Table 3.1 An exhaustive list of background effects and their relevance to this study. 51

Table 3.2 The raw materials of manufacture directly from GREET2 model and their 54 corresponding weights in kilograms.

Table 3.3 The types of fixed distributions used by SimaPro to model uncertainty 54 (adapted from Goedkoop et al., 2013).

Table 3.4 The total energy $(E)$ per vehicle in $\mathrm{kWh}$, standard deviation $(S D)$, standard error $(S E)$ and coefficient of variation $(C V)$ using regular and lightweight materials.

Table 3.5 The total energy $(E)$ per vehicle (sedan) in $\mathrm{kWh}$, standard deviation $(S D)$, standard error $(S E)$ and coefficient of variation $(C V)$ according to geographic location. 
Table 3.6 Comparison of total energy of vehicle (cradle-to-gate) from other studies. 66

Table 3.7 A breakdown of the total energy $(E)$ of a regular vehicle (sedan) in $\mathrm{kWh} 69$ and in terms of individual materials of manufacture $(i)$.

Table 3.8 A breakdown of the total energy $(E)$ of a lightweight vehicle (sedan) in 69 $\mathrm{kWh}$ and in terms of individual materials of manufacture $(i)$.

Table 3.9 A breakdown of the total energy $(E)$ of a regular vehicle (sedan) in kWh 69 and in terms of individual materials of manufacture $(i)$ by geographical region.

Table 3.10 Log transformed version of Table 3.9.

\section{CHAPTER 4}

Table 4.1 An updated list of materials used in Greenhouse gases, Regulatory Emissions, and Energy use in Transportation 2 as of 2012.

Table 4.2 Weight in kg of individual materials in Greenhouse gases, Regulatory 85 Emissions, and Energy use in Transportation 2.

Table 4.3 Weight in kg of individual plastics used in Greenhouse gases, Regulatory 85 Emissions, and Energy use in Transportation 2.

Table 4.4 Weight in kg of materials specific to fuel cell vehicles used in Greenhouse gases, Regulatory Emissions, and Energy use in Transportation 2.

Table 4.5 Time over which the available data on energy intensity $(\mathrm{kWh} / \mathrm{kg})$ of individual materials spans.

Table 4.6 Average energy totals, standard deviations and coefficient of variation for various power train configurations $(\mathrm{kWh})$.

Table 4.7 Means and standard deviations of materials for regular and lightweight sedans with different power trains $(\mathrm{kWh})$.

Table 4.8 Means and standard deviations of materials for regular and lightweight sedans with different power trains $(\mathrm{kWh})$.

Table 4.9 Means and standard deviations of fuel cell specific materials for regular 99 and lightweight sedans $(\mathrm{kWh})$. 
Table 4.10 Table of kurtosis values for each material.

Table 4.11 Table of skewness values for each material.

Table 4.12 Regression analysis for energy intensities of materials of manufacture using least absolute regression and least squares regression methods.

Table 4.13 Number of years for a one percent change in energy intensity $(\mathrm{kWh} / \mathrm{kg}) .113$

Table 4.14 A comparison of total energy per vehicle before and after log transformation $(\mathrm{kWh})$. 


\section{LIST OF FIGURES}

Page

\section{CHAPTER 1}

Figure 1.1 A visual overview of temporality in LCA Studies. 5

$\begin{array}{lll}\text { Figure } 1.2 & \text { Overall Structure of the Dissertation. } & 14\end{array}$

Figure 1.3 GREET1 and GREET2 models as adapted from 16

Tan et al. (2004b).

\section{CHAPTER 2}

Figure 2.1. Life cycle assessment framework adapted from ISO (1997). 32

Figure 2.2 The ISO 14040 standards in relation to other ISO platforms at 32 the time of their development (adapted from Fet, 1998).

Figure 2.3 LCA from the design perspective (adapted from

Gasafi et al., 2003).

\section{CHAPTER 3}

Figure 3.1 An overview of Chapter 3 and the Monte Carlo simulation. 50

Figure 3.2 Raw data of historic energy intensity $(\mathrm{kWh} / \mathrm{kg})$ for primary steel. $\quad 60$

Figure 3.3 Historical raw data of energy intensity $(\mathrm{kWh} / \mathrm{kg})$ of 60 primary aluminum.

Figure 3.4 An example of a single Monte Carlo distribution for the total energy of an internal combustion engine sedan.

Figure 3.5 A sample probability distribution for the energy intensity of copper. $\quad 70$

Figure 3.6 A sample cumulative probability distribution for the energy 70 intensity of copper. 
Figure 3.7 A one-way ANOVA of an internal combustion engine sedan using regular materials.

Figure 3.8 A one-way ANOVA of an internal combustion engine sedan using lightweight materials.

Figure 3.9 Histogram of PET energy of a regular sedan for the Five Year Period 73 (2007-2012).

Figure 3.10 Histogram of PET energy of a lightweight sedan for the Five Year Period (2007-2012).

Figure 3.11 Energy intensity $(\mathrm{kWh} / \mathrm{kg})$ for steel over various time intervals.

Figure 3.12 Countries studied for temporality due to age of data.

Figure 3.13 Countries involved in the spatiality analysis.

\section{CHAPTER 4}

$\begin{array}{lll}\text { Figure 4.1 The structure of the study for Chapter } 4 . & 87\end{array}$

Figure 4.2 An example Monte Carlo distribution for an all electric vehicle. 95

Figure 4.3 An example Monte Carlo distribution for a lightweight all electric 95

Figure 4.4 Box plots of an analysis of variance results for an all electric vehicle. 97

Figure 4.5 Box plots of an analysis of variance for an all electric vehicle 97

Figure 4.6 Box plots of an analysis of variance for a lightweight all electric vehicle. 98

Figure 4.7 Box plots of an analysis of variance for a lightweight all electric 98 vehicle (plastics only).

Figure 4.8 Box plots of an analysis of variance results for an internal combustion 101 engine vehicle.

Figure 4.9 Box plots of an analysis of variances results for an internal combustion 101 engine vehicle (plastics only).

Figure 4.10 Box plots of analysis of variance results for a lightweight hybrid electric vehicle. 
Figure 4.11 Box plots of analysis of variance results for a lightweight hybrid electric vehicle (plastics only).

Figure 4.12 Box plots of an analysis of variance results for a hybrid electric vehicle. 103

Figure 4.13 Box plots of an analysis of variance results for a hybrid electric vehicle 103 (plastics only).

Figure 4.14 Box plots of analysis of variance results for a lightweight hybrid electric 104 vehicle.

Figure 4.15 Box plots of analysis of variance results for a lightweight hybrid electric 104 vehicle (plastics only).

Figure 4.16 Box plots of an analysis of variance results for a plug-in hybrid electric vehicle.

Figure 4.17 Box plots of an analysis of variance results for a plug-in hybrid electric 105 vehicle (plastics only).

Figure 4.18 Box plots of an analysis of variance results for a lightweight plug-in hybrid electric vehicle.

Figure 4.19 Box plots of an analysis of variance results for a lightweight plug-in hybrid electric vehicle (plastics only).

Figure 4.20 Box plots of an analysis of variance results for a fuel cell vehicle.

Figure 4.21 Box plots of an analysis of variance results for a fuel cell vehicle (plastics only).

Figure 4.22 Box plots of an analysis of variance results for a fuel cell vehicle (fuel cell materials).

Figure 4.23 Box plots of an analysis of variance results for a lightweight fuel cell vehicle.

Figure 4.24 Box plots of an analysis of variance results for a lightweight fuel cell vehicle (plastics only).

Figure 4.25 Box plots of an analysis of variance results for a fuel cell vehicle (fuel cell materials). 


\section{CHAPTER 1-INTRODUCTION}

\subsection{Life Cycle Assessment: An Outline}

The central purpose behind the development and use of Life Cycle Assessments (LCA) was to promote informed decisions in the design and manufacture of products and services in order to assess their overall environmental impacts. This design tool has allowed for the introduction of design modifications such as the substitution of materials and methods in manufacturing so that more cost-effective and green-oriented products and services are brought about. However, the limitations of the process of LCA studies are mired in an ongoing debate over a multitude of facets within LCA methodology and principles. These problems include the issues of allocation, impact categories and streamlining processes. One issue not significantly addressed is that of the potential temporal effects ("distortions") on the validity of the results derived from LCA studies. What existing published material there is on this subject centres on the issue of the long-term effects of various emissions (carbons, $\mathrm{NO}_{\mathrm{X}}, \mathrm{SO}_{\mathrm{X}}$, etc.) characterized by their GWP (global warming potential) and, in turn, how they are discounted into present valuations. Consequently, the total sum of work in this area focuses on a narrow facet of the externalities arising from the output (sink) stage of the LCA. What has received little attention surrounds the input (source) stages of an enclosed industrial process. These latter input effects arise from temporal shifts within resource bases, technology dynamics, system complexity, system efficiency and alterations to environmental services. In this sense, it will complement discussions on uncertainty and variation in LCA literature. It is hypothesized that hitherto studies affected by temporality will have a much shorter longevity than previously assumed.

The process by which environmental impacts are quantified is termed environmental assessment (EA). LCA is one of the influential methodologies in environmental assessment. This methodology enables the quantification of environmental impacts of products and services across their entire lifespan, ranging from the raw material extraction/reuse, manufacture, usage and final disposal/recycling. In sustainability studies, the terminology most frequently used to describe 
this cycle is "cradle-to-grave" or, when more vigorous recycling is employed, "cradle-to-cradle." The main reason behind the usage of LCA is to gauge the environmental burdens and resources used, as well as examine design alternatives (Cooper and Fava, 2006). Consequently, the purpose of LCA may be seen to augment existent internal information regarding certain processes (Ibid.). A succinct definition of what this entails is given by Dreyer et al. (2006):

The product system encompasses all the processes involved in the different stages of the product's life from the extraction of raw materials, through manufacture, use and maintenance, to the final disposal of the product. When the focus is on environmental impacts, there is a natural link between the physical input or output of a process and a change in quality of the surrounding environment. The performance of the processes is thus the main driver behind the product's environmental impacts [...] (p. 89)

Nevertheless the balance of material dealing with inputs and outputs within LCA is slanted towards the output phases of industrial processes.

During the last twenty years, LCA has seen a growing presence within both the field of environmental studies and amongst practitioners. LCA has made possible the assessment of both manufactured goods and services. In doing so, it has allowed for the rationale of business decisions and design modifications for less environmentally deleterious outcomes. Furthermore, LCA has facilitated more holistic approaches to environmental issues. For example, economic analysis of environmental problems is hampered by difficulties associated with valuation and "market failures," while ecotoxicology solely examines the ecological impacts-that is, the end products - of anthropogenic activities. Nevertheless, the process of LCA has undergone some modifications during the last twenty years and has been largely codified by the International Organization for Standardization (ISO) under the ISO14040:2006 document Environmental management-life cycle assessment_principles and framework. Consequently, LCA is broken down into four separate but distinct stages: 


\section{Goal Definition and Scope}

This phase of the LCA process determines the type of information that will inform and augment the decision making process. Consequently, this step can be used to determine the resources needed and, in turn, how the resources will shape the final results. This is also useful in establishing the required specificity and how the data should be organized and the results displayed. Lastly, the ground rules of how to go about the work can be established.

\section{Life Cycle Inventory (LCI)}

The inventory is the part of the overall process which seeks to quantify and account for the entirety of each aspect of a product or service lifecycle from raw material extraction and processing, through to all the externalities such as emissions, releases and wastes. The LCI serves as the basis for the determination of environmental impacts and/or design improvements. Typically, this process includes developing a flowchart of the unit processes that make up the total product or service. This is followed by forming a strategy to collect data and subsequently followed by the actual collecting of data. Lastly, the results of the data collection are evaluated and reported.

\section{Life Cycle Impact Assessment (LCIA)}

The impact assessment phase of an LCA attempts to assess whether there may be a link between a product or service and its potential for environmental impacts. At this particular phase, it may be possible to establish the difference between products by their different potential impacts. The stages of the LCIA involve the selection and definition of impact categories, classification of impact categories, characterization of LCI results, normalization of variables, grouping or sorting different categories, weighting the

potential impacts and, lastly, reporting and evaluating the final results. Classification of impact categories involves assigning LCI result to impact categories such as global warming, acidification, etc. On the other hand, characterization of LCI results involves modeling the impacts with categories by science-based conversion factors. 


\section{Life Cycle Interpretation}

Life cycle interpretation is the final phase of the entire LCA process. At its core, interpretation involves explaining and communicating conclusions. This could involve quantitative (statistics) or qualitative analysis (such as valuation, preference, weighting, etc.). Study limitations and recommendations should be included in this phase as part of an overall process of transparency.

Nevertheless, the process of LCA continues to be encumbered by a number of drawbacks which call into question the validity of some aspects of the process and findings. This includes the fact that the data used in LCA is spatially and temporally dependent. This work seeks to address this knowledge gap in a normative sense with regard to energy intensity.

\subsection{Sources and Sinks}

Hofmeister and Kümmerer (2009) note that the separation of inputs and outputs during a process allows for their characterization as sources and sinks. Figure 1.1 presents a graphic representation of this is more detail. In turn, this has led to the treatment of inputs and outputs as two wholly separate parameters or factors with no relation to each other (Ibid.). For example, in Yuan et al. (2009), temporality was studied in relation to the environmental performance of an automobile with its production of greenhouse gases (GHGs) during its lifecycle. In this case, a performance evaluation was conducted using deterministic data rather than a stochastic distribution since the preliminary qualities of the study precluded the complexity involved in the latter case. All the sequential time frames in the study were added together, beginning with raw materials and continuing on to manufacturing, usage and, finally, disposal. Discount rates were pegged at five and ten percent (see Figure 1.1). Discount rates may be explained as the rate required by the Treasury Board of Canada for discounting future costs and benefits from public projects. Thus, the higher the discount rate, the more diminished will be the future costs and benefits derived from public projects. The use of discount rates in LCA is intended to augment the decision making process. In Steen (1997), the issue of temporality was solely in relation to GHG emissions - in this case, those coming from $1 \mathrm{~kg}$ of newspaper. Here, a linear relation of 
Figure 1.1 A visual overview of temporality in LCA Studies

\section{Inputs/Sources}

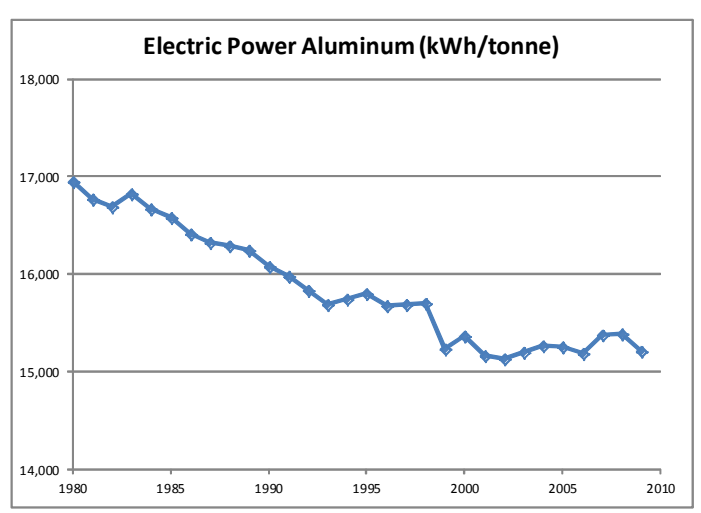

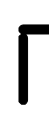
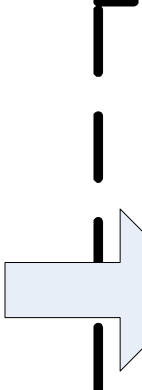

Note: For the most part, the extant work on the issue of temporality in LCA focuses on the impact of emissions on GWP (Global Warming Potential) indicators. The centre of the diagram is a where the LCA traditionally focused on a steady-state, cradle-to-grave system. The right hand side of the diagram illustrates that temporality has been traditionally characterized as an output or sink. The plot on the right side is taken from Yuan et al. (2009), which shows the trajectory of cumulative $\mathrm{CO}_{2}$ emissions over a twelve year period according to various discount rates. What have been ignored are the inputs or sources which enter the LCA from the left side of the figure. What is shown here is a temporal decrease in the electric power requirements (kWh/tonne) from 1980 to 2010 (International Aluminum Institute, 2011). Obviously, this would have significant ramifications for an LCI whose impact indicators include power usage. 
inputs was used instead of a non-linear relation, in spite of the inherent inaccuracies involved in the former. Coupled with this was the use of scenario analysis wherein one scenario involved recycling and the other incineration. Steen (1997) claimed that the uncertainty arises both from the use of data which is flawed in itself and from the aging of the database which holds the data. Also implicated in this promulgation of uncertainty was the problem of allocation (Steen, 1997), which relates to how impacts are assigned to individual processes in manufacturing processes which have multiple outputs. While it is usually preferred that allocation issues be consigned to a mass basis, other parameters such as weight, volume, (market) value, energy and demand can be used (Curran, 2007). In yet another example employing GHG, Huijbregts (1998b) examined temporality according to future GHG impacts arising from two different designs of a roof gutter. Along with scenario analysis, probabilistic modeling was used in his model. This was based on a matrix method originally proposed by Heijungs (1994, 1996). Lastly, Leroy and Froelich (2010) performed an LCA to study the GWP for both high-density polyethylene (HDPE) and lowdensity polyethylene (LDPE) grocery bags in order to study uncertainty propagation. They employed a mixture of qualitative methods and quantitative methods (Monte Carlo simulation) in their study.

As mentioned earlier, temporality has been studied because of its ability to track chemical fate and aid in risk analysis, although risk analysis is not the same as LCA. For example, Hertwich et al. (2000) studied the potential doses of 336 different chemicals from emission sources, including both wet and dry deposition. They used a model developed by Finkel (1990) for the analysis of uncertainty in risk assessment and risk management. Key to the model framework was the distinction of uncertainty arising from the model, decision rules and the input parameters, the last of which are also characterized as subject to variability. For example, variability characterizes combustion fuels in that they release energy somewhere within the lower and higher heating values (LHV/HHV). At any rate, Hertwich et al. (2000) employed three different sets of input parameters, each of which were characterized by both uncertainty and variability. The data sets included chemical specific data, landscape data and exposure data. A dominant exposure route was determined for each of the chemicals released into the air. For the 336 different chemicals, two sets of Monte Carlo analyses were conducted, such that one set 
employed the variance in chemical-specific parameters and the other employed the variance in all three parameters listed above. Then a sensitivity analysis was performed which evaluated the contribution to variance of each input parameter which ranged from 0.5 to 3.0 orders of magnitude in variance, but in most cases only 1 order of magnitude. The main reason for citing this work is that it employed a viable method for addressing uncertainty and variability which will be applied in this study with LCA. Lastly, Huijbregts et al. (2006) related the emissions arising from product manufacture to their environmental impacts, specifically through the study of the release of halogens from plastics manufacture and their consequential implication in ozone depletion. Subsequently, Huijbregts et al. (2006) were able to demonstrate through LCA that the cumulative energy demands of a product during the course of its lifecycle are a useful indicator of their environmental impacts.

\subsection{Uncertainty and Variance}

Uncertainty is implicated in many aspects of LCA. As defined in ISO 14040:2006 (s. 3.33), uncertainty analysis is a "systematic procedure to quantify the uncertainty introduced in the results of a life cycle inventory analysis due to the cumulative effects of model imprecision, input uncertainty and data variability." Embedded in this notion is that LCA implies some level of "value judgments" (Mining, Minerals, and Sustainable Development, 2002, p.275). For example, in the case of metals, there is "uncertainty over the specification and integration of impact categories, the relative importance of different environmental impacts, and the boundaries of impacts over both time and space" (Ibid.). Mining, Minerals, and Sustainable Development (2002) go so far as to claim that temporality is not an issue with metal LCAs since the physical state of metals never declines or alters. Rather, they contend that metals are subject only to impurities and can be recycled indefinitely. Because of claims such as these, traditionally "model uncertainties, such as a lack of temporal and spatial variability in the assessment, have not been quantified in LCAs" (Huijbregts, 1998b, 350).

Uncertainty in LCA studies is a broad topic eliciting a variety of opinion and methodology from practitioners. The most salient feature of this ongoing debate deals with the origins and types of uncertainty. Indeed, since the first Society of Environmental Toxicology and Chemistry 
(SETAC) workshop on uncertainty can be traced back to 1992 (Heijungs and Huijbregts, 2004), it is puzzling that, among other things, there has been no agreement on the origins and principles of general concepts such as uncertainty and variability. How broad or disparate this debate is may be elicited from the variety of opinions from the various works in Table 1.1. Furthermore, according to Heijungs and Huijbregts (2004), part of this confusion over what are uncertainty, variability and sensitivity is due to the lack of definition standardization for these terms, institutional or otherwise. An attempt to distinguish uncertainty from variability was made by these two authors:

Uncertainty relates to a lack of knowledge: no data is available, or the data that is available is wrong or ambiguous. Variability, in contrast, is a quality of data that is essentially of a heterogeneous nature. (p. 333)

An earlier attempt was made by Huijbregts (1998a) to explain the difference between uncertainty and variability as follows:

Variability is understood here as stemming from inherent variations in the real world, while uncertainty comes from inaccurate measurements, lack of data, model assumptions, etc. that are used to "convert" the real world into LCA outcomes. (p. 273)

The lack of agreement in this area makes it necessary in this study for the establishment of a high degree of specificity with regard to these terms and how they are elicited during the study of both LCA and temporality.

For example, according to Huijbregts (1998a), uncertainty in LCA can arise from the model, from practitioner choices, spatial considerations, temporality, and "variability between sources and objects" (276). For example, model uncertainty arises out of situations where there is a lack of inclusion of spatial and temporal characteristics within the LCA. Uncertainty due to choices occurs within an LCA when there are invariably choices made by necessity. These can include choices of weighting, allocation and methodology. Spatial uncertainty relates to emissions or 
Table 1.1 Types of Uncertainty (by study author).

\begin{tabular}{|c|c|c|c|c|c|c|c|}
\hline & & & & & \multirow{2}{*}{$\begin{array}{l}\text { Morgan \& Henrion (1990) } \\
\text { Hofstetter (1998) }\end{array}$} & & \\
\hline & & US EPA (1989) & Funtowicz \& Ravetz (1990) & Bevington \& Robinson (1992) & & Pohl et al. (1996) & Huijbregts (1998a) \\
\hline & Model & $x$ & $x$ & & & $x$ & $x$ \\
\hline & Choices & & & & $\mathrm{x}$ & & $\mathrm{x}$ \\
\hline & Spatial & & & & $\mathrm{x}$ & $\mathrm{x}$ & $\mathrm{x}$ \\
\hline & Temporal & & & & $\mathrm{x}$ & & $\mathrm{x}$ \\
\hline & Technology & & & & & $x$ & \\
\hline & Trajectory & & & & & & $\mathrm{x}$ \\
\hline \multirow{3}{*}{ Parameters } & Lack of data & $\mathrm{x}$ & $\mathrm{x}$ & & & $\mathrm{x}$ & $\mathrm{x}$ \\
\hline & Data inaccuracy & $x$ & $\mathrm{x}$ & & & & $\mathrm{x}$ \\
\hline & Unrepresentative data & $\mathrm{x}$ & $\mathrm{x}$ & & & & $\mathrm{x}$ \\
\hline & Epistomology & & & & & & \\
\hline & Mistakes (Calculation) & & & & $\mathrm{x}$ & $\mathrm{x}$ & \\
\hline & Metadata & & & & & & \\
\hline & Scenario & $\mathrm{x}$ & & & & & \\
\hline & Completeness & & $\mathrm{x}$ & & & & \\
\hline & Systematic & & & $\mathrm{x}$ & & $x$ & \\
\hline & Random & & & $x$ & $\mathrm{x}$ & & \\
\hline & Statistical methodology & & & & $\mathrm{X}$ & & \\
\hline & Linguistics & & & & $\mathrm{x}$ & & \\
\hline & Disagreement & & & & $\mathrm{x}$ & & \\
\hline & Ambiguity/Vagueness & & & & & $\mathrm{x}$ & \\
\hline
\end{tabular}

\begin{tabular}{|c|c|c|c|c|c|c|}
\hline & & Bedford \& Cooke (2001) & Huijbregts et al. (2001) & Björkland (2002) & Heijungs \& Huijbregts (2004) & Baker \& Lepech (2009) \\
\hline & Model & $x$ & & $x$ & & $x$ \\
\hline & Choices & $x$ & & $x$ & & $x$ \\
\hline & Spatial & & & $x$ & & \\
\hline & Temporal & & & $x$ & & $x$ \\
\hline & Technology & & & & & \\
\hline & Trajectory & & & $x$ & & \\
\hline \multirow{14}{*}{ Parameters } & Lack of data & $x$ & $x$ & $x$ & $x$ & $x$ \\
\hline & Data inaccuracy & $x$ & $x$ & $x$ & $x$ & $x$ \\
\hline & Unrepresentative data & $x$ & $x$ & $\mathrm{x}$ & & $x$ \\
\hline & Epistomology & $x$ & & $x$ & & \\
\hline & Mistakes (Calculation) & & & $\mathrm{x}$ & & \\
\hline & Metadata & & & $\mathrm{x}$ & & \\
\hline & Scenario & & & & & \\
\hline & Completeness & & & & & \\
\hline & Systematic & & & & & \\
\hline & Random & $\mathrm{x}$ & & & & \\
\hline & Statistical methodology & & & & & \\
\hline & Linguistics & & & & & \\
\hline & Disagreement & & & & & \\
\hline & Ambiguity/Vagueness & $\mathrm{x}$ & & & & \\
\hline
\end{tabular}

Note: Some material partially adapted from Heijungs and Heijbregts (2004). Some types of uncertainty (from separate studies) were placed in similar categories where properties were prima facie similar. 
effluent and how potential geographic and physical aspects (e.g., wind velocity) might shape the fallout. Temporal uncertainty occurs when "...temporal variation over short time periods is not made operational in the inventory analysis" (p. 276) because it is incongruous with inventory data. Thus, the determination of timeframe is of the utmost importance. According to Huijbregts (1998a), variability between sources and objects arises when there are different technologies used in manufacturing the same product. As well, this situation arises when there are variations in local characteristics such as variations in the physical environment, human population, etc. (flora and fauna). For reasons of simplicity, in Table 1 this particular faculty is termed "trajectory". Other authors such as Huijbregts et al. (2001) simply divide uncertainty arising in LCA according to either a lack of data or inaccuracy of data, which are termed by some authors as "parameters". Björkland (2002) provides a more comprehensive list of uncertainties by adding to material from Huijbregts (1998a) and Huijbregts et al. (2001). This includes epistemological uncertainty which arises from the lack of knowledge of system behaviour. As well, Björkland includes mistakes in calculation, either before or during the LCA, and metadata errors. Some minor additions to this area were later made by Baker and Lepech (2009), which include model uncertainty, statistical/measurement error, uncertainty in preferences and uncertainty in a future physical system relative to the designed system. In the last case, this would include future changes in the system design.

Opinion on the methodology for dealing with uncertainty is also equally diverse. Huijbregts et al. (2001) state:

Lack of temporal, geographical and further technological correlation between the data used and needed may be accounted for by applying uncertainty factors to the non-representative data. (p. 128)

By non-representative data, Huijbregts et al. (2001) refer to data not captured because of spatial and temporal variability. Rather than variability, they also use the term "temporal correlation" (cited in Weidema (1998)) to express the degree of accordance of the data in the year the data was collected and the year the LCA study was conducted. Unfortunately, Huijbregts et al. (2001) contend that: 
Although a quantitative assessment of the uncertainty related to the use of unrepresentative data within an LCA study may be preferable, it is also extremely difficult. (p. 129)

What is meant by parameter uncertainty is explained by Huijbregts (1998a) as "empirical inaccuracy (imprecise measurements), unrepresentativity (incomplete or outdated measurements) and lack of data (no measurements)" (p. 274). In Table 1, this triad is intentionally cordoned off to reflect Huijbregts' contribution. Huijbregts (1998b) discusses this further and comments that choice of allocation, functional unit and environmental burdens has an effect on the overall uncertainty of the LCA study. Consequently, to compound the problem, "in practice it will be very difficult to underpin the uncertainty ranges for the huge number of parameters involved in the inventory analysis" (Huijbregts, 1998b, p. 350). Referring to Kennedy et al. (1996), in his earlier paper Huijbregts proposes that a complementary strategy to address this would be to "implement uncertainty ranges for accumulated environmental interventions rather than individual parameters in LCA inventories" (p. 350). Nevertheless, in a later paper, Huijbregts et al. (2001) proposed the following uncertainty factor correction formula:

$$
E_{x, k}^{\prime}=U F_{t, k} * U F_{g, k} * U F_{t, k} * E_{x, k}
$$

Where $E_{x, k}{ }_{x, k}$ is the corrected emissions of substance $x$ per unit process $k(\mathrm{~kg}) ; U F_{t, k}$ is the uncertainty factor representing the temporal correlation between the data used and needed for unit process $k$ (dimensionless); $U F_{g, k}$ is the uncertainty factor representing the geographical correlation between the data used and needed for unit process $k$ (dimensionless); $U F_{f t, k}$ is the uncertainty factor representing the further technological correlation between the data used and needed for unit process $k$ (dimensionless); and $E_{x, k}$ is the initial emission of substance $x$ per unit process $k(\mathrm{~kg})$. However, this proposed method of dealing with temporal conditions, which is based in classical uncertainty modelling, is somewhat dated. In addition, the same authors contend that the use of Monte Carlo simulations is a valid way of employing stochastic modeling to make data inaccuracies in LCIs operational. 


\subsection{Problem Identification}

The process of establishing an international standard for LCA studies saw the emergence of ISO14040 in 2006. While ISO14040 sets out the guidelines and requirements for conducting LCAs, it does so in broad terms while leaving the mechanics of the assessment up to the LCA practitioner. This dissertation identified and concentrated on a specific niche related to the requirements of ISO14040 which had not previously been systematically examined or assessed. As will be discussed at length in Chapter 3, the issues of temporality and spatiality are broad subject areas of concern in LCA. Björkland (2002) goes into more detail about the effects of temporal variability. He states that,

...variations over time are relevant in both the inventory and impact assessment, as processes and factors in the receiving environment vary naturally over short and long time scales. Examples are process emissions, wind speed, and temperature. Another aspect is the chosen time horizon to integrate potential effects, which, for instance, applies to global warming potentials (GWP), photochemical ozone creation potentials (POCP)..., and emissions from landfills... (p. 65)

This introduces a paradox in that LCA should move towards greater overall simplification while bringing about greater resolution to reduce uncertainty and variability:

...model simplifications are common and necessary in LCA. The use of non-linear models, dynamic models, and multi-media models can reduce model uncertainty and address temporal and spatial variability. (p.66)

To specifically address temporal variability, Björkland states that higher resolution models should include sensitivity analysis, uncertainty importance analysis, classical statistical analysis, Bayesian statistical analysis, and scenario modeling (to be discussed in Methodology). In summation, this study seeks to find whether the historic shifts in power consumption during the raw material acquisition and refining stages shift in a consequentially temporal manner so as 
to significantly affect the overall power consumption of manufactured goods. To this end, the following research questions will be addressed:

1) Are there issues of temporality and spatiality due to age of data topics meriting concern? If so, how will it be possible to ascertain this?

2) What is the best method for assessing temporal and spatial age of data? Should it be done at the level of the functional unit, or should it be done at the resource level; that is, at the production level (primary production)?

3) What types of methods should be utilized to specifically deal with temporality and spatiality due to age of data?

4) What lessons could be learned from this, and how do they apply to life cycle assessments?

\subsection{Research Objectives and Methodological Approach}

Figure 1.2 provides the overall structure of this dissertation. Consequently, it can be seen that both phases 2 and 3 build on previous work, with the overall aim of establishing a base platform on which potential future investigations and assessments of temporality and spatiality due to aged data may be possible. For the purposes of this study, aged data is defined as data which is rooted in a specific time (by year) and place (by country or geographic location). As the methods employed in this study are detailed in depth in their respective chapters, it will be the aim here to merely present an outline of the methods employed. Table 1.2 has also been provided as supplementary information to Figure 1.2.

\subsubsection{Model Choice}

Greenhouse gases, Regulatory Emissions, and Energy use in Transportation 2 (GREET2) is a freely available and complete LCA developed by the Argonne National Laboratory, US Department of Energy. GREET2 models the full life cycle (cradle-to-grave) of sedans, sport utility vehicles and pick-up trucks, as well as different power trains: internal combustion, 


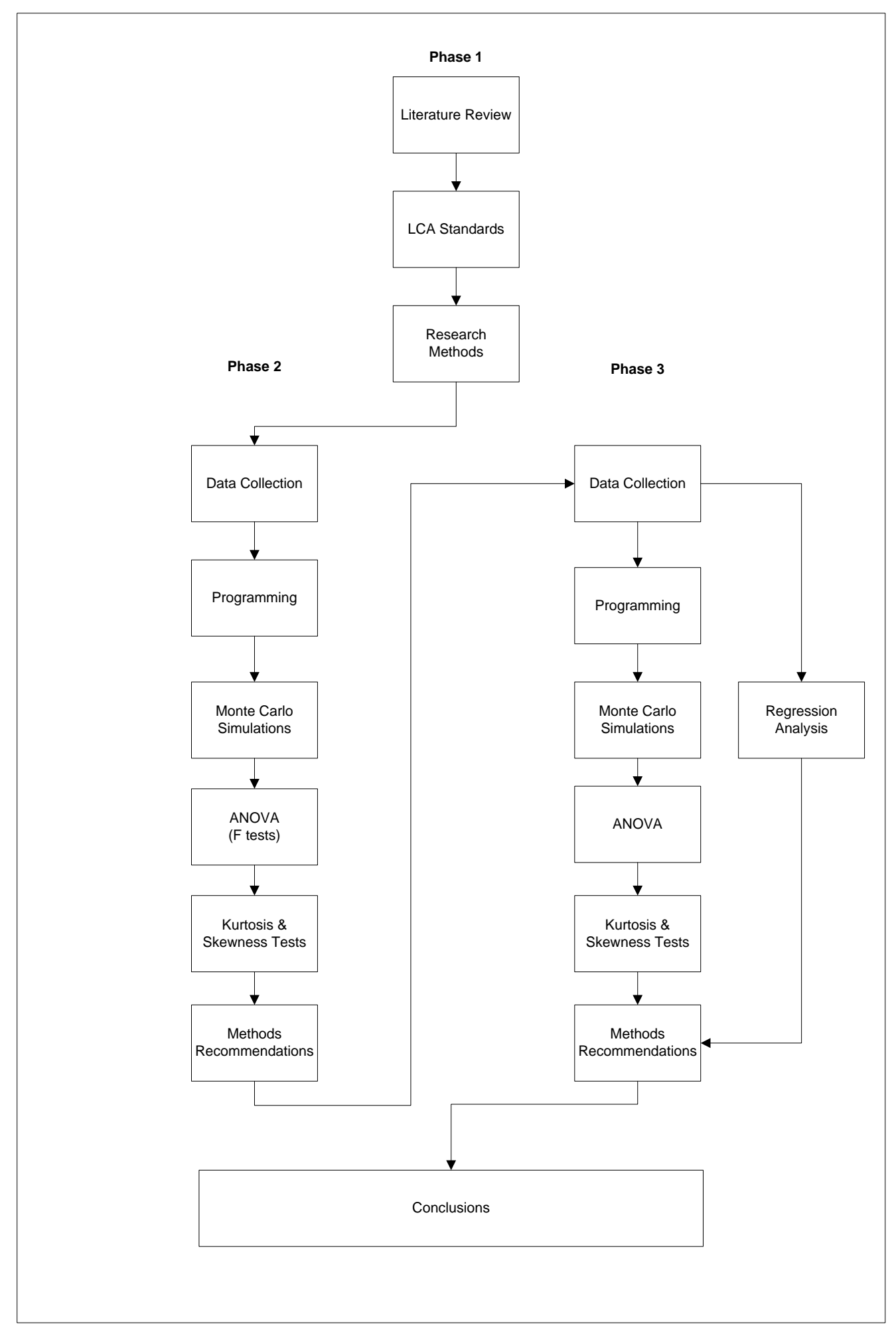

Figure 1.2 Overall Structure of the Dissertation 
Table 1.2 Details related to the simulation phases of the dissertation.

\begin{tabular}{|c|c|c|c|c|c|}
\hline Stage & $\begin{array}{l}\text { Number of } \\
\text { Raw Materials }\end{array}$ & Vehicle Formats & $\begin{array}{l}\text { Power Train } \\
\text { Configurations }\end{array}$ & $\begin{array}{l}\text { Regions } \\
\text { Covered }\end{array}$ & Methods \\
\hline Phase 2 & 17 & $\begin{array}{l}\text { Sedan } \\
\text { Sport utility (SUV) } \\
\text { Pick-up Truck (PUT) }\end{array}$ & ICEV & $\begin{array}{l}\text { Global } \\
\text { North } \\
\text { America } \\
\text { Europe } \\
\text { Oceania }\end{array}$ & $\begin{array}{l}\text { Monte Carlo } \\
\text { ANOVA1 } \\
\text { F-test } \\
\text { Kurtosis/skewness }\end{array}$ \\
\hline Phase 3 & 36 & Sedan & $\begin{array}{l}\text { ICEV } \\
\text { EV } \\
\text { HEV } \\
\text { PHEV } \\
\text { FCV } \\
\end{array}$ & Global & $\begin{array}{l}\text { Monte Carlo } \\
\text { ANOVA1 } \\
\text { Regression } \\
\text { Kurtosis/skewness }\end{array}$ \\
\hline
\end{tabular}

hybrids, plug-in hybrids, fuel cells and full electric vehicles. The purpose behind using this particular model was that the sponsoring agency behind GREET2 (US Department of Energy) had significant traction. Consequently, this brings confidence to this dissertation since GREET2 provides the weight of the individual materials of manufacture. Furthermore, each aspect of the life cycle of the vehicles is modelled on a gate-to-gate basis. Gates consist of distinct stages during a product's life cycle and can include raw material acquisition, ore refining, product assembly, use, and disposal. As such, this modularity allows for the breakdown of the model to the exclusion of unnecessary stages such as the usage portion of the life cycle, as well as the final disposal portion of its life cycle. Nevertheless, while there is considerable merit behind the GREET2 model, it must be noted here that the vehicles presented by the model are representative; that is, they are generic and do not refer to any specific make or model.

The first version of GREET (GREET1) was released in 1996 and has since been updated and expanded to version 1.8c. As may be seen in the horizontal loop of Figure 1.3, the primary purpose of this platform was to fully evaluate energy and emission impacts of advanced vehicle technologies and new transportation fuels, from the raw material acquisition stage (e.g., drilling) on through to its usage during the vehicle's operational lifecycle (combustion). The second series of GREET (GREET2) deals with the manufacturing aspects of the vehicle, also from raw material acquisition to the end of its lifecycle. Included in this model are configurations for the actual physical dimensions of the vehicle and lifecycle components to account for primary and secondary manufacturing. Contrary to other databases such as ecoinvent, this database is public 
domain knowledge and can be used by anyone. GREET is broken down into discreet modules (gates) dealing with the acquisition and processing of each raw material (metals, plastics, etc.). Power consumption, effluents, emissions and other outputs are simultaneously calculated and graphed accordingly. As a spreadsheet, GREET is designed so that alterations to inputs can be treated as discreet variables. However, the size and complexity of GREET in Excel is so unwieldy that modeling such as the Monte Carlo simulations has to be done externally in MATLAB. Consequently, only the framework of GREET will be used. Energy intensity of the various inputs $(\mathrm{kWh} / \mathrm{kg})$ with particular emphasis on metals with high energy requirements will be treated as the discreet variable in this instance.

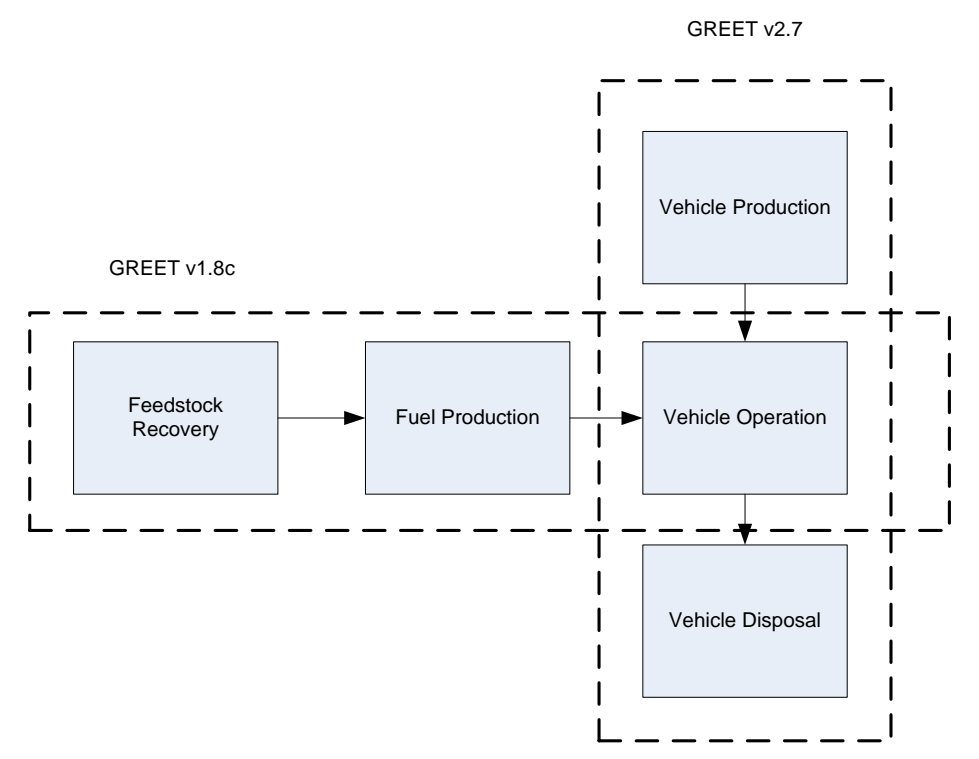

Figure 1.3 GREET1 and GREET2 models as adapted from Tan et al. (2004b).

\subsubsection{Data Collection}

Data collection on the energy intensity of materials for manufacture was a time-intensive process and took over a year to conclude for this dissertation. The particulars of why this process took so long was owing to the sheer volume of source documents (much more than are listed in the Data References appendix) as well as the amount of data contained within each of them. For example, individual source documents from the International Aluminum Institute and the International Iron and Steel Institute often contain data from all five continents which are furthermore broken 
down into multiple countries (see Figure 3.11 for a full visual breakdown of countries in this study). Naturally, because virtually all the source documents were in paper format, this required many hours of data entry. As well, often the units used in the source documents required very time consuming conversion, such as millions of British Thermal Units per pound (mmBTU/lb), barrels of oil equivalents (BOE) or tons variously expressed in United States tons $(907.2 \mathrm{~kg})$, United Kingdom tons $(1016.1 \mathrm{~kg})$ or metric tonnes $(1000 \mathrm{~kg})$. Another aspect implicated here is the fact that many of the early source documents were split into two separate documents, one specific to the manufacturing sector and another specific to the energy sector. For example, steel production in the European Union during the initial recorded decades (1960s and onwards) was often listed in one document by the amount per country (tons) while another document had to be referenced in order to get the total energy used specifically by the steel sector. Another recurring problem which was highly time intensive was owing to the source of data used in various published LCAs. In fact, often was the case that a published LCA did not use original source data, but used data from another published document. In turn, this second document also did the same and used data from yet another source. Consequently, a chain of as many as three or four papers would have to be back-checked to find the original source document and, as was often the case, the original source document was already entered into the database for this study. Lastly, with very few exceptions, many source documents published prior to the mid-1990s are not digitized. Thus, physical copies of these source documents had to be found. This was not particularly facile given that many of the physical copies of these documents are either archived, library collections are not always exhaustive and the institute with a physical copy is not willing to lend.

Data collection is an important facet of this study because, as a subset of the overall life cycle assessment process, life cycle inventory (LCI) is the aspect of LCA which seeks to quantify and account for the entirety of each aspect of a product or lifecycle from raw material extraction and processing, through to all the externalities such as emissions, releases and wastes. Thus, LCI serves as the basis for the determination of environmental impacts and/or design improvement. The process of data collection was exhaustive and drew on a diverse body of sources. As selection criteria, two particular qualities are relevant. Firstly, where possible, original 
documents were utilized in order to find their corresponding temporal and spatial origins. As mentioned earlier, often data used in some LCA studies were, in fact, far removed both spatially Table 1.3 Principle Classifications of Life Cycle Inventory Data.

\begin{tabular}{|l|l|}
\hline Type of Data & Definition \\
\hline Class I & Comprehensive data from direct measurements. \\
\hline Class II & Data from theoretical mass and energy balances. \\
\hline Class III & $\begin{array}{l}\text { Data from literature and databases. } \\
\text { Surrogate data. } \\
\text { Data estimated from experience. }\end{array}$ \\
\hline
\end{tabular}

Table 1.4 Breakdown of Types of Documents Used in Phase 3 of the Dissertation.

\begin{tabular}{|l|l|}
\hline Type of Document & Number \\
\hline International Databases & 151 \\
\hline Published Papers & 60 \\
\hline Congresses, Proceedings, Technical Papers & 44 \\
\hline Government Reports & 16 \\
\hline LCA/LCIs & 25 \\
\hline CSR Reports & 46 \\
\hline Books, Book Chapters & 26 \\
\hline
\end{tabular}

and temporally from their source and, hence, are non-representative. Secondly, given the technical scope of the subject, all the data used in this study came from primary and secondary sources to the virtually complete exclusion of all other potential sources. Hence, although there are non-governmental organizations interested in the energy profile of certain industries, it is certainly beyond their capabilities to provide the means for in situ measurements or access to data equivalent to in situ measurements. Certainly, the ability to generate this type of data is beyond the capabilities of anyone not attached to a research institute, university, corporation or representative industrial associations. Consequently, by the very nature of the type of data collected, the scope limitations on data exclusion from this study are fairly narrow. Referencing Tan et al. (2002), data collected could be classified according to all three principle classes of 
data, including direct measurements, theoretical mass and energy balances, as well as data from literature and databases (see Table 1.3). As well, the typology of documents used in this study were far reaching and attempted to be as exhaustive as possible. The types of documents and the amount utilized are given in Table 1.4. Phase 2 of this dissertation used 323 source documents, while Phase 3 used 368 documents, as is reflected in the totals of Table 1.4. While it can be seen that around $41 \%$ of the documents are from international databases, there is no one particular source which dominate the field of source material. Further detail regarding data collection can be found in Chapters 3 and 4.

\subsubsection{Monte Carlo Simulations}

Monte Carlo simulations are a probabilistic based method of analysis for the assessment of both variation and uncertainty. This method offers numerous advantages over extant methods of analysis. For example, sensitivity analysis typically examines end points, or highest and lowest values across a data range while largely ignoring patterns between these points. When using a linear membership function, the same can be said of fuzzy interval analysis. However, fuzzy interval analysis also introduces the problem of what particular membership function should be chosen in the first place. Often, as is the case, this choice appears largely subjective. When a probability distribution is available (sometimes this is not the case), Monte Carlo simulations can precisely draw statistical inferences across the entirety of the probability distribution. Monte Carlo simulations can do this by exactly mapping out the probability distribution with a large randomly generated data set. However, this comes with the trade-off of time intensity. Even with the latest series microprocessor (Intel Core i7 3630QM @ 2.40 GHz) and streamlined programming, each individual Monte Carlo simulation used in this study took up to two days to complete.

Consequently, Monte Carlo simulations have been central to the analysis of uncertainty in LCA (Hendrickson et al., 2006; Leroy and Froelich, 2010). As Baker and Lepech (2009) explain, this has consistently been one of the most popular methods. Monte Carlo simulation as a significant tool in the analysis of uncertainty in LCA is cited by Björkland (2002), Huijbregts et al., (2003), 
Heijungs and Frischknecht (2005), as well as Baker and Lepech (2009). As stated by Huijbregts (1998a):

Stochastic modelling, which can be performed by Monte Carlo or Latin Hypercube simulation, seems to be an especially promising technique for making uncertainty in model output operational. An advantage in relation to the other methods mentioned is that, dependent on the available information, various parameter distributions, such as uniform, triangular, normal [or Gaussian], or lognormal distributions, can be used in the model. (p. 274)

The use of various parameter distributions is repeated by Heijungs and Huijbregts (2004), as well as Heijungs and Frischknecht (2005), who claim that the choice is difficult as to which statistical distribution to use. However, in the case of Heijungs and Frischknecht (2005), no information how to go about this is provided. Consequently, to date only uniform and triangle distributions appear to characterize the available data. However, as will be discussed within Chapter 3, probability distributions were constructed based on available data (see below). That aside, Monte Carlo simulation takes the following form (Hendrickson et al., 2006, p. 48):

1. The underlying distribution, correlations, and distribution parameters are estimated for each input-output coefficient, environmental impact vector, and required sector output. Correlations refer to the interaction of the uncertainty for the various coefficients.

2. Random draws are made for each of the coefficients in the Economic InputOutput-LCA (EIO-LCA) model.

3. The environmental impacts are calculated based on the random draws.

4. Steps 2 and 3 are repeated numerous times. Each repetition represents another observation of a realized environmental impact. Eventually, the distribution of environmental impacts can be reasonably characterized.

Lo et al. (2005) have examined the potential for global warming effects of alternative municipal waste treatment strategies using a Bayesian Monte Carlo simulation. However, this case study 
was used strictly to quantify and reduce parameter uncertainty in LCA. It did not include "structural uncertainties" such as those arising from variability of input parameters and other models (Lo et al., 2005, 32). This can be useful when there is stochastic randomness in data sets.

While Monte Carlo simulations form the centrepiece of this study, other methods were considered at the start of this research. However, many of these methods had drawbacks that were decisive in their rejection. Bootstrap methods were considered, but there was no identified necessity for resampling from the data set. Fuzzy methods posed the problem of which type of membership function to choose. Furthermore, choosing a set distribution - as identified later in the text of Chapter 3.2.1 - is antithetical to the purpose of this study. Similarly, the same issue was apparent with Taylor series analysis since this would have to use an indeterminate number of Taylor series expansions in order to create the probability density functions (which appears to be a considerable amount of unnecessary work which is equally antithetical to the Fuzzy methods approach). Bayesian analysis was also rejected because there was no apparent requirement for posterior probabilities. EIO-LCA methods presented further difficulties with regard to accessing economic data and, as explained in Chapter 3.2.2, the data collection for this research was already considered excessively time-consuming. Lastly, sensitivity analysis was deemed to be substandard to Monte Carlo analysis since sensitivity analysis does not render as much statistical detail. Thus, Monte Carlo methods were chosen on the basis of their simplicity of computation (albeit with the trade-off that they are time-intensive) and the depth of statistical analysis facilitated.

\subsubsection{Analysis of Variation and F tests}

A one-way analysis of variance is a general method for analyzing data from designed experiments whose objective is to compare two or more group means. That is, there is only one independent variable. The object is to present the variance within groups that results from a single treatment (or, in this case, the mass of individual materials of manufacture which make up the functional unit or vehicle). As such, ANOVA1 makes possible the comparison of quantities by examining the differences between them (Weiss, 2005). The results of the ANOVA1 in this dissertation are presented as box plots so that visualizations of the variance resulting from Monte 
Carlo simulations can be explicitly examined. As such, it will provide a means for examining the degree to which each individual material of manufacture contributes to overall variance. For example, this would enable a comparison between how much variance steel contributed versus the amount of variance that polyvinyl chloride contributed within the functional unit. Because the functional unit has different masses of each of these materials, as well as different energy intensities of manufacture, the ANOVA1 will be able to answer this question by expressing the variance in terms of pure energy $(\mathrm{kWh})$.

Also used here is the F test, which is an ANOVA that examines the variance between groups (Ibid.). It does so by calculating the $\mathrm{F}$ value, which is the ratio of variances between groups to the variance within groups. This had import for the spatiality portion of this dissertation which tested for differences in energy distributions by geographic region. Consequently, it will be possible to see whether the differences in energy distributions by geographic area are significant or not. Naturally, higher F values would signal that these are significant and small $\mathrm{F}$ values would indicate that the group means are not reliably different. Whether the derived $\mathrm{F}$ values are significant or not is determined on the basis of the critical value associated with its significance level, or p-value. The critical level chosen for these studies was one percent since this has a lower threshold for rejecting insignificant differences (as opposed to the five percent significant level).

\subsubsection{Regression Analysis}

The purpose of regression analysis is to establish whether there exists a relationship between a dependent and independent variable. In this dissertation, regression analysis serves primarily as an ancillary method to the Monte Carlo simulations and ANOVA. Because Monte Carlo simulations are a probabilistic based method and ANOVA's purpose is to measure differences within and between groups, it was felt that the addition of regression analysis was useful to see whether the variance arising from both the probability distribution in the Monte Carlo simulations and ANOVA were due to actual temporal relations or the product of scatter data. Two types of regression analysis were utilized, both the least squares and the more robust least absolute residuals. Their utility is assessed as per their functionality in this type of study. 


\subsubsection{Tests for Non-Normal Data}

It was assumed in the early stages of this dissertation that there would be no instances of nonnormal data. Nevertheless, the ongoing research process had to give way to examining this subject owing to the particular shape (skew) of the distributions used in the Monte Carlo simulation, as well as the witnessed phenomenon of non-scalability. Consequently, this was an add-on during the later stages of the dissertation. As is well known, data which does not display normality has implications in establishing regression lines and correct mean values. In the case of the latter, non-normal data would require the use of log transformations in order to get correct means. The effects of non-normal data have ramifications for the findings of $\mathrm{F}$ tests (Markowski and Markowski, 1990), which is a method used in this dissertation.

\subsection{Glossary of Key Definitions}

Although somewhat intuitive, terminology relevant to the field of life cycle assessment has specific implications associated with their overall structure, functionality and design as outlined in Chapters 1.1 and 2.1. Consequently, a purposeful glossary of key definitions has been provided as supplementary material to the main body of the text. Unless specified, all terms are derived from the International Organization for Standardardization (ISO) document Environmental management_life cycle assessment_principles and framework (2006).

Allocation: Partitioning the input or output flows of a process or a product system between the product system under study and one or more other product systems.

Ancillary Input: Material input that is used by the unit process producing the product, but which does not constitute part of the product.

Characterization Factor: Factor derived from a characterization model which is applied to convert an assigned life cycle inventory analysis result to the common unit of the category indicator. 
Co-product: Any of two or more products coming from the same unit process or product system.

Cut-off Criteria: Specification of the amount of material or energy flow or the level of environmental significance associated with unit processes or product system to be excluded from a study.

Data Quality: Characteristics of data that relate to their ability to satisfy stated requirements.

Elementary Flow: Material or energy entering the system being studied that has been drawn from the environment without human transformation, or material or energy leaving the system being studied that is released into the environment without subsequent human transformation.

Energy Flow: Input to or output from a unit process or product system, quantified in energy units.

Functional Unit: Quantified performance of a product system for use as a reference unit.

Impact Category: Class representing environmental issues of concern to which life cycle inventory analysis results may be assigned.

Input: Product, material or energy flow that enters a unit process.

Intermediate Flow: Product, material or energy flow occurring between unit processes of the product system being studied.

Intermediate Product: Output from a unit process that is input to other unit processes that require further transformation within the system.

Life Cycle: Consecutive and interlinked stages of a product system, from raw material acquisition or generation from natural resources to final disposal. 
Life Cycle Assessment (LCA): Compilation and evaluation of the inputs, outputs and the potential environmental impacts of a product system throughout its life cycle.

Life Cycle Impact Assessment (LCIA): Phase of life cycle assessment aimed at understanding and evaluating the magnitude and significance of the potential environmental impacts for a product system throughout the life cycle of the product.

Life Cycle Interpretation: Phase of life cycle assessment in which the findings of either the inventory analysis or the impact assessment, or both, are evaluated in relation to the defined goal and scope in order to reach conclusions and recommendations.

Life Cycle Inventory Analysis (LCI): Phase of life cycle assessment involving the compilation and quantification of inputs and outputs for a product throughout its life cycle.

Output: Product, material or energy flow that leaves a unit process.

Process: Set of interrelated or interacting activities that transforms inputs into outputs.

Process Energy: Energy input required for operating the process or equipment within a unit process, excluding energy inputs or production and delivery of the energy itself.

Product: Any goods or service.

Product Flow: Products entering from or leaving to another product system.

Product System: Collection of unit processes within elementary and product flows, performing one or more defined functions, and which models the life cycle of a product.

Raw Material: Primary or secondary material that is used to produce a product. 
Reference Flow: Measure of the outputs from processes in a given product system required to fulfill the function expressed by the functional unit.

Releases: Emissions to air and discharges to water and soil.

Sensitivity Analysis: Systematic procedures for estimating the effects of the choices made regarding methods and data on the outcome of a study.

System Boundary: Set of criteria specifying which unit processes are part of the product system.

Spatiality: In life cycle assessments spatiality is a vast subject area and can refer to multiple phenomena such as:

- the geographic dispersion and spatial implications of pollutants such as emissions into the environment (Krewitt et al., 2001; Newell and Vos, 2011;

Dresen and Jandewerth, 2012)

- considerations specific to waste management (Ekvall et al., 2007)

- resource usage and extraction by geographic area (Pfister et al., 2009)

- spatial implications of production in different geographic areas (Steinberger et al., 2009)

- land use (Geyer et al., 2010; Saad et al., 2011)

- differences arising from using LCI databases based in different countries and geographic regions (Suh et al.. 2013)

Temporality: In life cycle assessments temporality is a vast subject area and can refer to multiple phenomena such as:

- Age of data utilized, the lifespan of a product (from cradle to grave), the use and renewal of resources (Klöpper, 2000)

- The timespan of the goal and scope definitions used in LCA (Phungrassami, 2008) 
- The dynamics of emissions to the environment (Levasseur et al., 2010; Säynäjoki et al., 2012; Levasseur et al., 2012)

- The temporal dependence of data (Fleisher et al., 2004)

- The flow of materials across time in Dynamic LCAs Hendrickson et al., 2006; Stasinopoulos et al., 2012; Beloin-Saint-Pierre et al., 2014)

Uncertainty: With regard to life cycle assessment, uncertainty relates to a lack of knowledge, be it the non-availability of data or that the data is wrong or ambiguous (Heijungs and Huijbregts, 2004). It can also refer to circumstances arising from inaccurate measurements, model assumptions, etc. which are used to "convert" the real world into life cycle assessment outcomes (Heijbregts, 1998a). For the central purposes of this study, uncertainty refers to the lack of available data.

Uncertainty Analysis: Systematic procedure to quantify the uncertainty introduced in the results of a life cycle inventory analysis due to the cumulative effects of model imprecision, input uncertainty and data variability.

Unit Process: Smallest element considered in the life cycle inventory analysis for which input and output data are quantified.

Variability: Relates to a quality of data such that the data is homogeneous in nature (Heijungs and Huijbregts, 2004). This means that data is both varied and dissimilar. This variability arises through innate variations in the real world (Heijbregts, 1998a). Hence, variability may represent the true diversity or heterogeneity of a properly representative population (U.S. Environmental Protection Agency, 1997).

Waste: Substances or objects which the holder intends or is required to dispose of. 


\subsection{Structure of the Dissertation}

This dissertation is organized around the four chapters as follows:

Chapter 2 (Life Cycle Assessment and the progress towards standardization and normalization) addresses Phase 1 of the research. The chapter is based partly on the paper:

Pryshlakivsky, J., Searcy, C.: 'Fifteen years of ISO14040: a review', Journal of Cleaner Production, 2013, 57, DOI: 10.1016/j.jclepro.2013.05.038

The primary author of the paper is Mr. Jonathan Pryshlakivsky. Mr. Pryshlakivsky's involvement in the development of the paper includes: primary research, concept development, exposition, and correspondence with the journal with regard to revisions and publication matters. As the secondary author, Dr. Cory Searcy's involvement with the development of this paper includes: identification of research viability, concept development, research supervision and review of the manuscript prior to publication. The goal of this paper is to identify the context of ongoing problems in the area of life cycle assessment and the research gap that this dissertation seeks to address. Hence, this chapter is the basis for moving forward in the critical areas developed in chapters 3 and 4 of this dissertation.

Chapter 3 (Analysis of how energy intensities from vehicle manufacturing materials vary with the age of data and the region they represent) addresses Phase 2 of the research. The chapter is based on a revised manuscript resubmitted for publication as follows:

Pryshlakivsky, J., Searcy, C.: 'Analysis of how energy intensities from vehicle manufacturing materials vary with the age of data and the region they represent,' Journal of Cleaner Production.

The primary author of the manuscript is Mr. Jonathan Pryshlakivsky. Mr. Pryshlakivsky's involvement in the development of the manuscript includes: primary research and data collection, computer programming, testing of programming (accuracy), methods development and recommendations. As the secondary author, Dr. Cory Searcy's involvement in the 
manuscript includes: supervision of the research process, and review of the paper for publication.

Chapter 4 (Monte Carlo simulations and regression analysis for assessing temporality due to age of data) addresses Phase 3 of the research. The chapter is based on a revised manuscript resubmitted for publication as follows:

Pryshlakivsky, J., Searcy, C.: 'Monte Carlo simulations and regression analysis of the effects of temporality due to age of data using the Greenhouse gases, Regulatory Emissions, and Energy use in Transportation 2 model,' Journal of Cleaner Production.

The primary author of the manuscript is Mr. Jonathan Pryshlakivsky. Mr. Pryshlakivsky's involvement in the development of the manuscript includes: data collection, computer programming, simulation running, regression analysis, methods development and recommendations. As the secondary author, Dr. Cory Searcy's involvement in the manuscript includes: supervision of the research process, and review of the paper for publication. This paper further explores concepts developed in Phase 2 of the research. It more than doubles the amount of materials of manufacture studied and broadens the level of analysis to include regression analysis with the aim to making recommendations regarding data updating in life cycle inventory. It also identifies problems and makes recommendations for future analysis in this area.

The dissertation closes with $\underline{\text { Chapter } 5}$ (Research Summary and Conclusions). This final chapter builds on the research phases that are presented in Chapters 2, 3, and 4: to summarize the results and list the key findings; to present the conclusions and recommendations; to identify the research limitations and areas for future research; and to review the contributions of the dissertation. 


\section{CHAPTER 2-LIFE CYCLE ASSESSMENT AND THE PROGRESS TOWARDS STANDARDIZATION AND NORMALIZATION.}

The purpose of this chapter is to contextualize the subject of life cycle assessment (LCA) and the emergence of the theme of this dissertation within the process of standardization. To this end, it is informative in illustrating just how nascent life cycle assessment is, the scope of problems posed in this area, and the progress made with LCA research methods towards more robust outcomes and performance. Concerted progress has been made in this area over a very short period of time (less than twenty years), including establishing methods for analysis of LCA objectives arising out of the standardization process.

\subsection{Overview of LCA Development and Standardization}

Life Cycle Assessment (LCA) is a methodology by which manufacturers or service providers can analyse the environmental impacts and effects of their products and services. The duration of this assessment extends across the entire life cycle of products and services ("cradle-tograve"). This process allows for product comparison and strategic decision making with regard to systemic inputs and outputs, as well as the development and incorporation of End-of-Life (EOL) design strategies. Environmental Management Systems (EMS) such as ISO 14001 provide a platform which guides the overall process towards achieving institutional environmental compliance. In a similar manner, the family of ISO 14040 standards frames the requirements for conducting Life Cycle Assessments (LCA) while leaving the actual mechanics of analysis - data collection, normalization, calculation, interpretation, etc. - to the practitioner. A systemized framework for conducting LCAs was released by the International Organization for Standardization (ISO) during the period 1997-2000, resulting in the standards ISO 14040, 14041, 14042 and 14043. Updates to these standards were completed in 2006 so that the previous standards were amalgamated into ISO 14040 and 14044. Certainly much comment and critique concerning LCA methodology itself is extant (e.g., see Reap et al., 2003). It is also reasonably well known in this field that full-scale LCAs are data and time intensive (Capello et al., 2008). 


\subsection{Pre-2006}

LCA standardization began at ISO Technical Committee (TC 207) Subcommittee SC 5 in Paris in November 1993 (Marsmann, 1997). The standard was directly inspired by the Code of Practice developed by SETAC, the Society of Environmental Toxicology and Chemistry (Lecouls, 1999). The need for these standards was based on a growing recognition "of the usefulness of LCA as a methodological tool for the continuous process in identifying environmental aspects within the framework of environmental management systems according to ISO 14001" (Ryding, 1999, p. 307). The now well-known four box framework of the ISO 14040 series took shape around four distinct stages which include the Goal and Scope Definition, Inventory Analysis, Impact Assessment and Interpretation (see Figure 2.1). Not surprisingly, these four stages informed the texts of each of the ensuing documents: 14040 General Principles in 1997, 14041 Life Cycle Inventory Analysis (LCI) in 1998, as well as both 14042 Life Cycle Impact Assessment (LCIA) and 14043 Interpretation in 2000 (Salmone et al., 2005). Internally, the structure of ISO 14040 follows the pattern established with ISO 14001 (i.e., Scope, Normative References, Terms and Definitions, and so forth). Another purposeful reason behind the drafting of the standard lay in its position vis-a-vis the overall ISO 14000 family of standards (see Figure 2.2). As may be seen, the ISO 14040 series was conceived as a supplementary tool of an overall environmental management system (EMS) platform centred on ISO 14001. It may be stated that a useful goal of ISO 14040 series is to inform ISO 14001 with regard to the latter standard's requirement of continuous improvement to maintain compliance with the standard. Subsequently, Figure 2.3 demonstrates how the results of an LCA can contribute to design modifications of an existing product. This feedback loop can make contributions towards eventual product or service certification in ISO 14040. The ISO 14041 section on inventory analysis may use standardized data sets for industrial processes. However, using a database of processes introduces a level of standardization which does not address questions arising from uncertainty with local conditions or natural variation (UNEP, 2005). This can have the effect of compiling systemic errors and result in unacceptable levels of variance between LCA practitioners. Therefore, this allowable flexibility of permitting LCA 


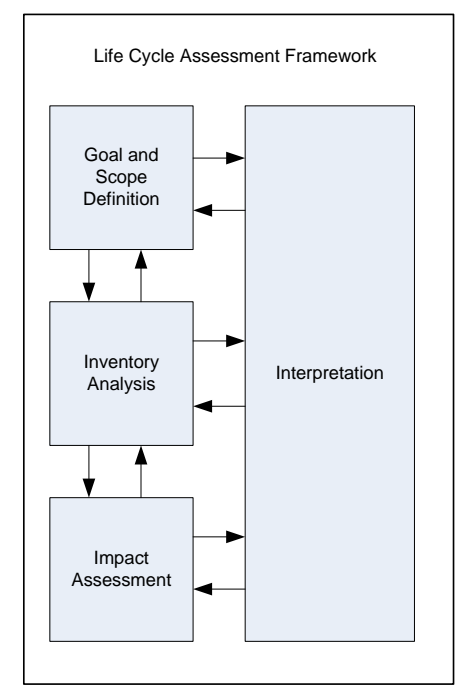

Figure 2.1. Life cycle assessment framework adapted from ISO (1997).

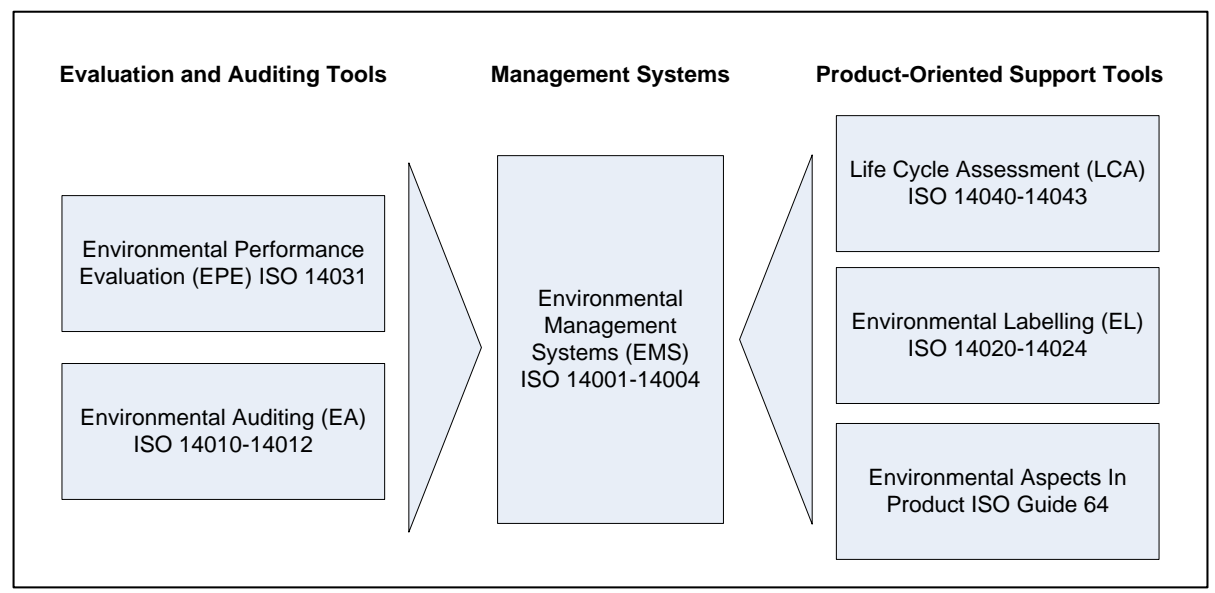

Figure 2.2 The ISO 14040 standards in relation to other ISO platforms at the time of their development (adapted from Fet, 1998).

practitioners to use whatever database they choose in the inventory analysis can produce different results, even when similar functional units are under consideration (Klüppel, 1998). As will be seen in Chapters 3 and 4, this is precisely the case since data taken from the same year can have widespread variance depending on location or technology used. In turn, this can have further consequences with regard to uncertainty arising from the determination of the effects of 


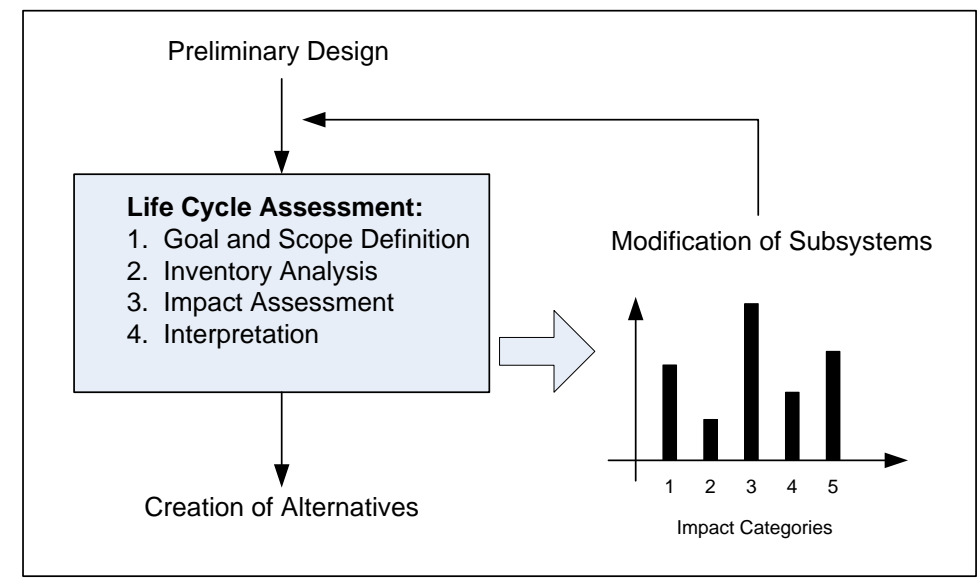

Figure 2.3 LCA from the design perspective (adapted from Gasafi et al., 2003).

compounding processes (Marsmann, 2000). This has pertinence to this study since there are implications for the data used in this study and how they are influenced by background processes (see Table 3.1). A similar sentiment was levelled against ISO 14042 that too much preference was given to the weighting (termed "valuation" in earlier documents) of impacts within impact categories (Udo de Haes and Jolliet, 1999; Ryding, 1999). The consequence of this could preferentially reduce the impacts of certain processes and introduce a degree of relativism into the LCA study (see Table 2.1 for a summary of critiques and whether the 2006 standard addresses them). So concerned with this problem was the German Federal Environmental Agency that they standardized this entire process in 1999 (Schmitz and Paulini, 1999). Ekvall and Finnveden (2001) also discuss this same problem with allocation in ISO 14041. Allocation arises when the complexity of a process results in multiple or different outputs (products) whose individual burdens are difficult to understand. However, Kim and Overcash (2000) noted that ambiguities in both the definition of unit process and system boundaries compound the problem of allocation and, hence, outcomes.

One aspect of the Life Cycle Inventory (LCI) variously discussed in lieu of ISO 14040 was its lack of accounting for "ecological effectiveness" and "ecological balances" of various comparative technologies (Bárzaga-Castellanos et al., 1999, p. 335). For example, a situation could exist where two products can have similar performance characteristics or functional units, yet one product has "least harmful option", it contributes little to measure the progress towards 
Table 2.1 Summation of pre-2006 critiques of the ISO 14040 series.

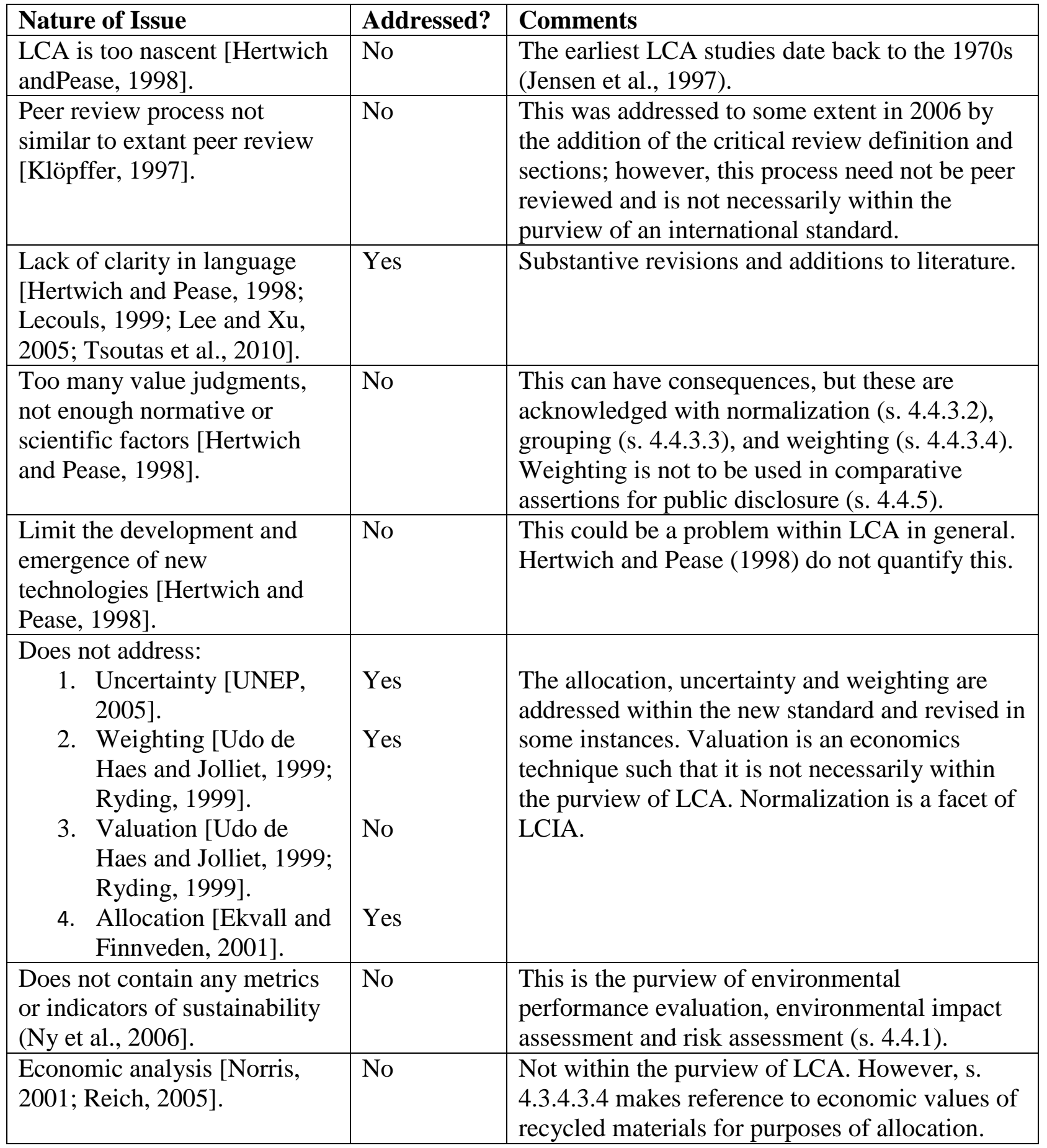


sustainability (Ny et al., 2006). Along this line, concerning methodology, Lee and Xu (2005, p. 22) state:

It has also been propositioned that LCA investigates burdens on the environment, rather than impacts because there is as yet no universally-acceptable method to assess impact on the environment. This may explain why current LCAs are also struggling with how to assess multiple product life cycles.

This was similarly expressed by Gasafi et al. (2003) in the context that the ISO 14043 standard did not establish a platform for identifying parameters which determine significant or remarkable effects. To address this, Gasafi et al. (2003) proposed the usage of a dominance analysis, even though after examination this appears to be remarkably similar in methodology to allocation itself. Nevertheless, as will be discussed later, there is a concerted need for ascertaining significance levels.

\subsection{Post-2006}

One of the chief aims of the new ISO 14040 series was "to provide a description of LCA principles and framework that is readable and accessible not only for LCA practitioners, but also a broader target audience" (Finkbeiner et al., 2006, p. 81). As mentioned earlier, the basis for the new series was a "principles and framework" document (ISO 14040), as well as a "requirements" document (ISO 14044) — that is, a "general guidance" and a "specific guidance" document. However, with this in mind, it should be stated here that ISO 14040 requires compliance with ISO 14044. Furthermore, as stated by Finkbeiner et al. (2006), it was crucial to the new series that comparative assertions be released to the public in order to protect third party reviewers and the potential misuse of comparative assertions.

It is quite noticeable that many sections of the ISO 14040: 2006 are copied verbatim from ISO 14040: 1997. This is in keeping with the objectives to maintain the original technical content, consensus and requirements (Finkbeiner et al., 2006). However, the differences are worth commentary. The new standard removed an entire section from the Introduction which dealt with the limitations of LCA techniques. These limitations included (ISO, 1997): 
- the nature of choices and assumptions made in an LCA, inventory analysis and environmental impacts;

- how local conditions can incur a level of relativism;

- the limitations imposed by data availability; and

- how the lack of spatial and temporal dimensions in the LCA adds to the level of uncertainty in the impact assessment.

Instead, the LCA limitations in the new document are relegated to an element of the scope of the international standard (s.1). That is, the standard makes no specific reference to these limitations. As all of these limitations have impacts for this study, it may be interesting to know where specifically in the standard do these issues become addressed. Not surprisingly, they now fall under the purview of data quality requirements (s. 4.2.3.6). Hence, in so doing, it will be seen that this dissertation becomes largely an issue of data quality.

Perhaps most remarkable are the number of additions to the s.3 definitions. The original standard had only twenty definitions (left column), while the new standard has forty-six. Some of the noteworthy additions include a definition for "product", which is here defined as any goods or services. Unlike other definitions in the standard, the definition of product includes several examples of what constitutes goods or services. In general, the additions increased the amount of physical characteristics (Material) and methodology (Method) while making substantial revisions to boundary characteristics. These revisions included a broadening of the analysis process to include elements such as uncertainty and sensitivity analyses. More weight was also given to account for the system contributions to goods and services. Also, there are a number of new terms which add a higher degree of specificity to the new platform. Among others, these would include specifications for endpoints and impact effects. The definitions for "allocation" and "waste" were revised and expanded, while only the term "practitioner" was dropped altogether from the new standard.

Even after the implemented changes in 2006, there is still some concern whether ISO 14040 is being used correctly (Cooper et al., 2008). Indeed, Table 2.2 contains a comprehensive list of 
Table 2.2 Summation of Recommendations Since 2006 and Their Potential Viability.

\begin{tabular}{|c|c|}
\hline Recommendations & Viability \\
\hline Toxicity assessment [Eckelman et al., 2008]. & $\begin{array}{l}\text { Not within the purview of LCA. Classically, } \\
\text { this has been the function of risk assessment. }\end{array}$ \\
\hline \multicolumn{2}{|l|}{ Functional unit: } \\
\hline $\begin{array}{l}\text { 1. ISO standard data quality criteria are } \\
\text { difficult to meet when presented with } \\
\text { complex systems such as farms which } \\
\text { have a diversity of inputs resulting in } \\
\text { the same output (e.g., potatoes) [Usva } \\
\text { et al., 2009]. } \\
\text { 2. Significant increases in functionality } \\
\text { [Collado-Ruiz and Ostad-Ahmad- } \\
\text { Ghorabi, 2010]. } \\
\text { 3. Functional unit inconsistencies, } \\
\text { including unforeseen uses of the } \\
\text { functional unit [Moberg, 2010]. } \\
\text { 4. Arbitrary in certain circumstances } \\
\text { such as pavement [Santero et al., } \\
\text { 2011a]. }\end{array}$ & $\begin{array}{l}\text { 1. Section } 4.2 .3 .6 .2 \text { makes a number of } \\
\text { data quality requirements including } \\
\text { consistency, reproducibility, as well as } \\
\text { precision (variance) and uncertainty. } \\
\text { Hence, this must be documented } \\
\text { where implicated in methodology, the } \\
\text { model studied and the assumptions } \\
\text { made. } \\
\text { 2. Ostensibly, this would result in a new } \\
\text { functional unit. However, this may be } \\
\text { a useful amendment to system } \\
\text { expansion rules under allocation. } \\
\text { 3. Full LCAs may not always be } \\
\text { practical or required when making } \\
\text { comparative assertions. } \\
\text { 4. Points } 1 \text { and } 3 \text { are implied here. Also, } \\
\text { results should be scalable when based } \\
\text { on a unit of weight [e.g., kWh used to } \\
\text { treat one litre of sewage]. }\end{array}$ \\
\hline $\begin{array}{l}\text { Stifles creativity which necessitates pragmatic } \\
\text { system process adjustments in order to bring } \\
\text { about synergistic benefits [Sheate, 2010]. }\end{array}$ & $\begin{array}{l}\text { In principle, the nature of LCA is supposed to } \\
\text { be iterative such that it may inform changes in } \\
\text { the study scope (see s. } 4.2 .1 \text { ). Additionally, } \\
\text { part of the purpose of LCA is to inform } \\
\text { design modification [see Gasafi et al., 2003]. }\end{array}$ \\
\hline \multicolumn{2}{|l|}{ Allocation: } \\
\hline $\begin{array}{l}\text { 1. The application of the ISO rules of } \\
\text { allocation are either not applicable or } \\
\text { they do not present easily discernible } \\
\text { choices. The authors were not able to } \\
\text { avoid allocation such that it broke } \\
\text { down or expanded the system but } \\
\text { chose to allocate environmental } \\
\text { burdens based on the inputs or } \\
\text { outputs, primarily through economic } \\
\text { value [Aver et al } 20071\end{array}$ & $\begin{array}{l}\text { 1. As per s. } 4.3 .4 .2 \text {, the requirement that } \\
\text { a partition be made which reflects } \\
\text { underlying physical relationships is } \\
\text { not elaborated. Supplementary best } \\
\text { practices might be a useful addendum } \\
\text { to this area. }\end{array}$ \\
\hline $\begin{array}{l}\text { 2. Carbon footprint is difficult to } \\
\text { establish [Finkbeiner, 2009]. }\end{array}$ & $\begin{array}{l}\text { 2. Depends on the system boundaries } \\
\text { and environmental significance of } \\
\text { process. }\end{array}$ \\
\hline
\end{tabular}


3. Allocation based on the absence of physical relationships (e.g., economic) do not result in information on the et al., 2011].

4. System expansion as per allocation is made murky when there is no definitive end-of-life scenario: in this case, old pavement is used as a base for new pavement [Santero et al., 2011b].

5. Not always possible to stick with one method of allocation when conducting a study. Physical results are useful for one audience, economic for another [Svanes, et al., 2011]. consequences of human actions [Renó

3. This issue is largely intractable since economic valuation, preference, etc., can be value-based.

4. This is addressed to some extent in s. 4.3.4.3.2 where reuse and recycling of a product implicates more than one product system (this must be "elaborated"). Similarly, alterations in product properties must be "taken into account". None of these address the uncertainty inherent in this situation.

5. This relates to the requirement that allocation methods be "uniformly applied to similar inputs and outputs in the system under consideration" (s.4.3.4.2). This promotes adherence to s. 4.2.3.6.2 regarding data quality.

\section{Critical review:}

1. Independence not required [Chanaron, 2007].

2. Insufficiency of detail regarding the critical review [Chomkhamsri et al., 2011].

May result in studies with no basis in science [Elcock, 2007].

\section{Ecology:}

1. No account of biological causality [Schau and Fet, 2008].
1. This is in keeping with the principles of standards as "soft law."

2. Expansion of this area (s. 6) seems credible.

This is acknowledged as a "limitation" in LCIA. However, it may not be a part of the Goal and Scope of the study to find scientific answers. Also, as stated in s. 4.4.3.3, ranking is based on value-choices, as is weighting (s. 4.4.3.4). This may be necessary when reflecting the preferences of individuals or groups in a strategic decision making platform.

1. Depends on system boundaries and cut-off criteria (s. 4.2.3.3), especially if they are of "environmental significance." Also, whether an LCIA is the stated purpose of the study [see Udo de Haes et al., 2002]. 


\begin{tabular}{|l|l|}
\hline $\begin{array}{l}\text { 2. } \begin{array}{l}\text { Does not account for ecological goods } \\
\text { and services, the impacts of renewable } \\
\text { resource usage, or make comparisons } \\
\text { against biocapacity and availability } \\
\text { [Fiksel, 2010]. }\end{array} \\
\begin{array}{l}\text { Persistency of uncertainty even with } \\
\text { adherence to the standard [Leroy and } \\
\text { Froelich, 2010]. }\end{array}\end{array} \begin{array}{l}\text { 2. Some of this may be implicated in } \\
\text { LCIA [see Udo de Haes et al., 2002]. } \\
\text { rules of data quality (s. 4.2.3.6.2) and is a } \\
\text { pervasive issue regardless of the area of } \\
\text { study. }\end{array}$ \\
\hline $\begin{array}{l}\text { Cut-off rules are redundant in current LCA } \\
\text { practices (because unit processes are } \\
\text { standardized in software) [Weidema et al., } \\
\text { 2008]. }\end{array}$ & $\begin{array}{l}\text { Their inclusion (s. 4.2.3.3) should be } \\
\text { maintained owing to ongoing concerns with } \\
\text { temporal and spatial variability. }\end{array}$ \\
\hline $\begin{array}{l}\text { System boundaries entirely up to the } \\
\text { discretion of the analyst [Whitehead et al., } \\
\text { 2010]. }\end{array}$ & $\begin{array}{l}\text { According to s. 4.3.3.4, the system boundary } \\
\text { must be based on significance as per a } \\
\text { sensitivity analysis. }\end{array}$ \\
\hline
\end{tabular}

further recommendations for expanding the standard. Yet, perhaps the most positive element to come out of the revision process is that the new ISO 14040 series has coincided with the maturation of the LCA studies (Parker et al., 2007). Some have recently gone so far as to state that the ISO 14040: 2006 series "has proved a suitable tool for sustainability assessment" (Sablayrolles et al., 2010, p. 232). Fava et al. (2009) have claimed that ISO 14040 should be the basis for future LCA studies. Indeed, the growing public presence of ISO 14040 is worth noting. Klöpffer (2009) reported an LCI study of the aluminum smelting operations of the European Aluminium Association (EAA). This assessment, which was kept almost entirely within the parameters of the new ISO 14040 series, facilitated incremental environmental performance improvements over the time period of years 2000 to 2008. Nevertheless, a number of concerns of varying gravity still persist (see Table 2.2). Heijungs et al. (2010) have gone so far as to state that the classical model of LCA should be dispensed with in order to render a more broadly based format for assessment that takes into consideration both the empirical and normative attributes of LCA and the concomitant process of standardization. This is contrary to the original document where "under ISO guidelines for LCA, normalization is conceived of as taking place at the stage of impact assessment" (Upham et al., 2011, p. 353). 
Thus, it may be seen that some wish to extend the standard's functions into other areas such as ecology, economics, etc. This opinion coincides with a sentiment that the standard is lacking in the degree of specificity. For example, one study of nanotechnology found that the new ISO series was valuable in its application to this particular field but that the series did little to tackle issues of toxicity assessment (Eckelman et al., 2008). While changes were made to how different products are compared in the new standard, there are still problems how to go about a comparison when functionality is significantly enhanced (Collado-Ruiz and Ostad-AhmadGhorabi, 2010). Other concerns regarding the functional unit still centre on its presumed lack of definition or potential for ambiguity, in spite of the fact that the functional unit by definition must be quantified. Similar sentiments also pervade the issue of allocation. In many of these cases, there appears to be the absence of a metric or hermeneutic which brings about clear choices that can be readily identified in a classical decision making framework (Ayer et al., 2007; Renó et al., 2011; Santero et al., 2011b; Svanes et al., 2011; Elcock, 2007; Whitehead et al., 2010). It appears unlikely these issues will be resolved owing to the inherent complexity of many systems approaches and, as noted by Leroy and Froelich (2010), the persistence of uncertainty. There remains concern whether the standard contributes to stifling creativity when bringing about synergistic benefits in systems (Sheate, 2010). In principle, the iterative nature of LCA and informed design modifications (Gassafi et al., 2003) should reduce the potential for stifling creativity. It is apparent that there is a lack of detail with regard to the critical review portion of the standard, and this probably should merit some attention at some future time. Also of importance here is the issue of a lack of ecological consideration for causality, biocapacity, renewable resource usage and environmental goods and services (Schau and Fet, 2008; Fiksel, 2010). A not inconsiderable amount of attention has been given to this subject in LCIA (e.g., see Udo de Haes et al., 2002), yet ISO 14040 does not outline how to deal with these sorts of problems unless they have "environmental significance." On the other hand, it would be correct to also claim these areas as the purview of a broader field such as industrial ecology. However, with that in mind, Upham et al. (2011) assert that LCA "methods...have become increasingly codified and some have been commercialised, so that complete standardization is now unlikely and in some respects not even desirable" (p. 353). 
Klöpffer (2009) reported an LCI study of the aluminum smelting operations of the European Aluminium Association (EAA). This assessment, which was kept almost entirely within the parameters of the new ISO 14040 series (except for the critical review), facilitated steady environmental performance improvements over the time period of years 2000 to 2008. The steady improvements took the form of energy savings and emissions reductions. The aluminum refining industry has been involved with the ISO 14040 series since its inception in 1997 owing to the high energy intensities (sometimes termed "embedded energy") involved in converting alumina $\left(\mathrm{Al}_{2} \mathrm{O}_{3}\right)$ to pure aluminum (de Schrynmakers, 2009; Klöpffer, 2009). Consequently, there is a vested economic and environmental interest in the aluminum industry to see the refining process improved. Because aluminum presents such difficulties in refining in terms of required energy (e.g., see the comparative study done by Norgate et al., 2007), a high degree of aluminum recycling is usually evidenced in manufacturing because of the potential for reducing production costs and environmental burdens (de Schrynmakers, 2009). The allowance for system expansion and material substitution in ISO 14044 is of particular importance since it allows aluminum LCA practitioners a high degree of necessary flexibility, especially with regard to allocation methods and system boundary definitions (European Aluminium Association, 2007). This is reflected in the new standard by the purposeful changes to some definitions. For example, Product System saw the phrase "materially and energetically connected unit processes" changed to "unit processes with elementary and product flows" (ISO, 1997, ISO, 2006a). This allowed for the differentiation between virgin materials ("elementary flows") and recycled materials ("product flows") as is necessary for aluminum recycling. Likewise, the definition of System Boundary was changed so that it no longer needs to be between the product system and the environment. Rather, the new definition allows the practitioner the ability to choose which unit processes make up the System Boundary. Certainly, this makes sense in the case of aluminum recycling since the initial production stages of mining bauxite and chemically reducing alumina are to be excluded. Similarly, the definition of waste was altered to accommodate the recycling process by narrowing the definition from "any output" to materials or objects which the producer intends or is required to dispose of. 


\subsection{Conclusions}

In summary, this chapter has outlined that the ongoing issue with LCA and standardization is a balancing act between greater calls for more specificity (which necessitates more robustness) and the flexibility of methods. Naturally, this problem largely turns into a trade-off issue since more specificity comes at the expense of flexibility and vice versa. As will be shown in Chapters 3 and 4, greater specificity also introduces a number of problems-mainly in the form of complexity - beyond the scope of simple trade-offs.

Certainly, in its Requirements and guidelines document, ISO14040 does state in section 4.2.3.6 that a number of data quality requirements should be addressed. The first relates to time-related coverage, which includes the age of data and the minimum length of time over which data should be collected. The second data quality requirement which should be addressed is the geographic coverage, or the geographical area from which data for unit processes should be collected to satisfy the goal of the study. In addition to this, the following statement is made further on in the standard in section 4.5.1.2 dealing with interpretation of results:

[...] uncertainty is introduced into the results of an LCI due to the compounded effects of input uncertainties and data variability. One approach is to characterize uncertainty in results by ranges and/or probability distributions. Whenever feasible, such analysis should be performed to better explain and support the LCI conclusions.

This passage has pertinence to this study in that Monte Carlo simulations will be used to construct probability distributions. But, this is also pertinent as part of the ISO14040 requirements, which include a sensitivity check and a consistency check. The purpose of the sensitivity check (s. 4.5.3.3) is to assess reliability of the LCA final results, while the consistency check (s. 4.5.3.4) determines the consistency of methods, assumptions, and data. Consistencies include both the representativeness of age of data, as well as the geographical coverage (s. B.3.4). In the appendix of the requirements document (s. B.3.3), the sensitivity for variations in data can be expressed in terms of a range, percentage of change, or absolute deviation. 
Consequently, it can be seen that ISO14040 requires that the issue of temporality and spatiality due to age of data be a subject to be documented and assessed. However, these assessments are both internal and for individual LCAs. They are neither a systematic analysis of these issues, nor an examination of methods. Nevertheless, it may be seen that the basis for the ensuing chapters finds its roots within the ISO14040 standard, with its simultaneous desire for more robust explanations and conclusions to LCA studies. 


\section{CHAPTER 3 - ANALYSIS OF HOW ENERGY INTENSITIES FROM VEHICLE MANUFACTURING MATERIALS VARY WITH THE AGE OF DATA AND REGION THEY REPRESENT}

\subsection{Introduction}

Life cycle assessment (LCA) is a cradle-to-grave approach to assessing industrial systems by presenting a comprehensive view of environmental aspects associated with products and services. As such, LCA makes possible informed decision making with regard to trade-offs and selection of processes (Scientific Applications International Corporation, 2006). LCA also facilitates comparisons between alternative functional units (Fleet et al., 2000). However, purposeful decision making is rooted in the accuracy and completeness of information. Life Cycle Inventory (LCI) databases have been characterized by a lack of completeness such that the corresponding impacts of this deficiency bring into question the reliability of overall LCA findings (Khasreen et al., 2009). Indeed, utilizing six different databases to perform an LCA on polyvinyl chloride, Peereboom et al. (1999) found significant quantitative differences in certain impact categories owing to data uncertainty. Further discussion on the subject of uncertainty will follow below in the Methods section.

One particular area that calls into question the dependability of decisions and outcomes in LCA is the effect of temporality and spatiality on study findings. Classically, temporality issues within LCA were ignored because of concerns regarding economy (cost), information security and the cumbersome nature of centrist (top-down) management organizations (Schaltegger, 1996). Similarly, the inclusion of temporality in LCA poses a problem with regard to data collection since suppliers lack environmental performance data (Ibid.). As stated by Udo de Haes (2006), LCA is a steady-state tool due to the fact that all processes have different time characteristics. However, as Reap et al. (2003) pointed out, the consideration of a steady state activity in an industrial process has little connection with events which happen in an ecosystem where the primary effects (externalities) are time dependent. As suggested by Field et al. (2001), perhaps the reasoning behind this trend stems from the fact that the inclusion of temporality into 
LCA would necessitate the consideration of not only a single product (functional unit), but the sum total of all products whose emissions enter an ecosystem. Naturally, for the purposes of LCA, this may not be feasible given the high number of individual products whose emissions collectively enter a given ecosystem.

As a subject area within LCA, temporality is broad. According to Held and Klöpffer (2000), time related issues in LCA may consist of: the age of data; the period of use of products (duration); the time scales of recycling; the time horizons of ecological effects (impact assessment); the time scales of exploitation of non-renewable resources; the regeneration rate of renewable resources; and the time horizon for waste management, landfills, etc. In the case of the last group, this can also relate to toxicity (acute versus long-term), nuisances (e.g., noise), radiation decay, global warming, photochemical smog, acidification, and so forth (Ibid.). Time can also be involved in the time frame of the goal and scope definition, as well as the LCI from resource extraction, to manufacture, to end of life stages, further on to landfill emissions (Phungrassami, 2008). Naturally, this extends to the time period over which the impacts are integrated in LCIA (Ibid.). Temporality may also relate to the interrelations between processes in a product system, the profile of the emissions arising from the product system, as well as the dynamic characteristics of the impacted areas (Pinsonnault et al., 2014). These are further elaborated below.

Previous consideration of temporality within LCA studies has been in regard to the definition of the system boundaries so that it took into account atypical system behaviours such as downtime, set-up and/or fluctuations in input materials volume flows (Ciambrone, 1997). Accordingly, this was adjudged to last no longer than between nine and twelve months. However, by and large, according to Goedkoop et al. (1998) and Udo de Haes et al. (2004), temporal and spatial information is lacking in almost all LCAs. Similarly, Graedel (1998) notes that "analytical approaches that tend to emphasize temporal difference tend to be discounted" (p. 74). The shortcomings of LCA with regard to temporality and spatiality have been noted in several works (Reap et al., 2003, 2008a, 2008b). As stated by Reap et al. (2008b), the most important aspect of temporality and spatiality was that potentially important industrial dynamics were ignored (Reap et al., 2008b). Furthermore, they stated that traditional life cycle assessment could not account for environmental and industrial dynamics because of a lack of dynamic modeling or historical 
data (Ibid.). For example, anthropogenic emissions have their own time scale, as do their associated impacts (Collet et al., 2011). To address this, Owens (1997) states that characterizing toxicity of particular activities necessitates that, whether true or not, all emissions must be accounted for under the assumption that they will cause effects. Thus, within the LCA, threshold limits are eliminated and a linear dose-response is assumed. The reason for this rendering of time-dependence into simultaneous exposure (or aggregation) is the ability to consider what may be deemed a worst-case scenario of exposure to potentially toxic substances. However, this has had the effect of producing inaccuracies owing to the compressed time scale in the inventory analysis and the subsequent results/findings (Levasseur et al., 2010).

The domain of LCIA has utilized time more explicitly than any other dimension of LCA, particularly in the characterization and normalization steps (Phungrassami, 2008). Here, characterization models the impacts of LCI by using science-based conversion factors in impact categories and normalization entails facilitating comparison of potential impacts (Scientific Applications International Corporation, 2006). Therefore, it is perhaps not surprising to find that ISO 14040:2006 Environmental management_Life cycle assessment_Principles and framework makes specific reference in section 4.3(i) to the non-inclusion of temporality (ISO, 2006a): LCA addresses potential environmental impacts; LCA does not predict absolute or precise environmental impacts due to:

- the relative expression of potential environmental impacts to a reference unit,

- the integration of environmental data over space and time,

- the inherent uncertainty in modeling of environmental impacts, and

- the fact that some possible environmental impacts are clearly future impacts

In this instance, this means the aggregation of effects occurring over space and time. Most extant temporality studies in LCA focus strictly on the output of greenhouse gases (GHGs) and their associated global warming potential (GWP) (Levasseur et al., 2010; Säynäjoki et al., 2012; Levasseur et al., 2012). In this instance, GWP is the cumulative radiative force arising from the emission of a unit mass of a greenhouse gas (GHG) within a specific time horizon and measured against $\mathrm{CO}_{2}$ (Levasseur et al., 2010). This recent work was necessary owing to the fact that 
traditional LCA studies ignored the temporal profile of emissions (Säynäjoki et al., 2012; Levasseur et al., 2012).

In many instances, the extant LCA literature focuses solely upon the issue of temporality from the perspective of an externality. In this way, temporality is thought of as an adjunct of the central LCA. Thus, inputs into the system which are characterized by temporality are not currently well defined. Hofmeister and Kümmerer (2009) note that the separation of inputs and outputs during a process allows for their characterization as sources and sinks. In turn, this has led to the treatment of inputs and outputs as two wholly separate parameters or factors with no relation to each other (Ibid.). Temporality is also implicated in the dynamics of material availability. At any given time in a production phase, the amount of virgin material available (stocks and inventory) can vary according to how much similar recycled material is available and at what cost. Consequently, a dynamic analysis of material flows in a temporal dimension has to be incorporated with a feedback loop through the series of unit process models (Hendrickson et al., 2006). As may be suspected, this has further implications for process truncation and system boundaries as system complexity increases. This has been the subject of some research in the area of dynamic LCAs. For example, Stasinopoulos et al. (2012) considered the temporal impacts of new products entering and old product exiting from a fleet of vehicles. Beloin-SaintPierre et al. (2014) performed a dynamic analysis by linking together elementary unit processes. Dynamic LCAs, however, have nothing to do with this study as the data under study here is composed of discreet data points in space and time. Thus, the system of manufacture is internally static even though changes in energy intensity may be attributed to efficiency improvements or changes in technology.

The age of data relates to the temporal dependency of data Fleisher et al. (2004) and, as such, constitutes the major focus of temporality in this study. Perhaps this was not originally thought to be a considerable problem as indicated by De Smet and Stalmans (1996) because every LCI practitioner uses the best available data at the time of the study and, if necessity requires it, the study could be updated at a future time when new data becomes available. Age of data issues have been previously characterized through the use of data quality indicators, vectors, and characterization factors (Weidema and Wesnaes, 1996; Kennedy et al., 1997; Pinsonnault et al., 
2014). However, as this consists of the addition of metadata, the problem of age of data is not being addressed but only characterized. As such, the metadata is purely a qualitative metric and not a quantitative measurement of the depth of the problem, which is an aim of this study. Timeseries studies such as Memary et al. (2012) which consider solely the impacts on LCA findings of time-varying parameters in historical data are rare.

Studies of spatiality largely mirror that of temporality, although the breadth of pollutants is not restricted to emissions (Ekvall et al., 2007; Steinberger et al., 2009; Helmes et al., 2012). Most of these studies deal with the "end of pipe" spatial implications arising from emissions (Krewitt et al., 2001; Newell and Vos, 2011; Dresen and Jandewerth, 2012). Only in one instance is there spatial consideration of waste management (Ekvall et al., 2007). However, Pfister et al. (2009) do consider the spatial implications at the input stages of production as they relate to water consumption resource extraction by geographical region. Also, Steinberger et al. (2009) compare the energy consumption spatial implications of production in India versus China, as well as product usage in Germany. Therefore, only in the case of Steinberger et al. (2009) does this study bear some semblance, although the breadth of materials and time frame under consideration are much larger. Also, efforts to address spatiality issues in LCA historically exceed that of temporality issues. On the one hand, these studies can be divided mainly into spatiality issues related to emissions (Nigge, 2001; Potting and Hauschild, 2006; Roy et al., 2013). On the other hand, they may refer to land use (Geyer et al., 2010; Saad et al., 2011). Only Suh et al. (2013) deal with the spatial implications arising from different LCI databases located in different continents (Europe, North America, Asia, etc.). As such, none of these papers have implications for this study.

One subject area which must be discussed in light of this study is the effect of background processes which may or may not contribute to differences in energy intensity data. Some differences were found in data on similar processes originating from separate databases, as well as whether a process-based or economic input-output based approach was pursued for compiling and reporting the data (Peereboom et al., 1999; Mongelli et al., 2005; Miller and Theis, 2006). The most notable differences in the above studies were seen in the output elements of the LCA relating to emissions. Remarkably, with all three of these above studies, the differences in the 
input data of the LCA were found to be relatively small (as compared with outputs). Only a study by Takano et al. (2014) saw a mixed quality to the input data. A study by Cellura et al. (2011) also tested the gross energy requirements for a Sicilian tile utilizing data from three different databases. Like Takano et al. (2014), there were smaller differences emerging from the input data $( \pm 7.6 \%)$ while output data differences ranged from inconsequential to substantial. However, some of the causes of differences found in data are not applicable to this study, especially those relating to emissions profiles, while others do have relevance. That is, their relevance lies in that the effects of these causes are precisely what are being measured in this study. To this end, Table 3.1 provides explanations of which of these causes have relevance (duplicates of causes were eliminated from later papers for succinctness).

In summary, issues with temporality due to age of data and spatiality have largely omitted the study of input materials in LCA. It is an aim of this study to present a general overview at the subject of temporality and spatiality in LCA. In doing so, this allows for the context of this study to be understood. The sector focus of this study is mainly the vehicles field and the materials for their manufacture. It is also an aim to fill a knowledge gap that has hitherto been unstudied. Further to this end is to examine whether there are any significant effects arising out of temporality and spatiality due to age of data. It does so by analyzing the effects of changes in total energy for internal combustion engine vehicles (ICEV) composed of regular and lightweight materials of manufacture. Compiling and utilizing a vast body of literature and data, this study examines whether this problem merits further attention. In accordance with this will be the development of methods for dealing with these issues and bringing about more reliable results to LCA.

\subsection{Methods}

An overview of this study is provided in Figure 2.1. As such, this figure augments the text of this chapter. Processes (calculations) are represented by rectangular boxes while parallelograms consist of data, whether raw or the result of a specific process. It may be noted that the components which comprise the Monte Carlo simulations are in the central column. It consists of the pseudo-random number generator which creates the random uniform distribution below 
this (in the parallelogram), as well as the reproduction of the probability distribution at the bottom of the column. Thus, all other processes are ancillary to the Monte Carlo simulation.

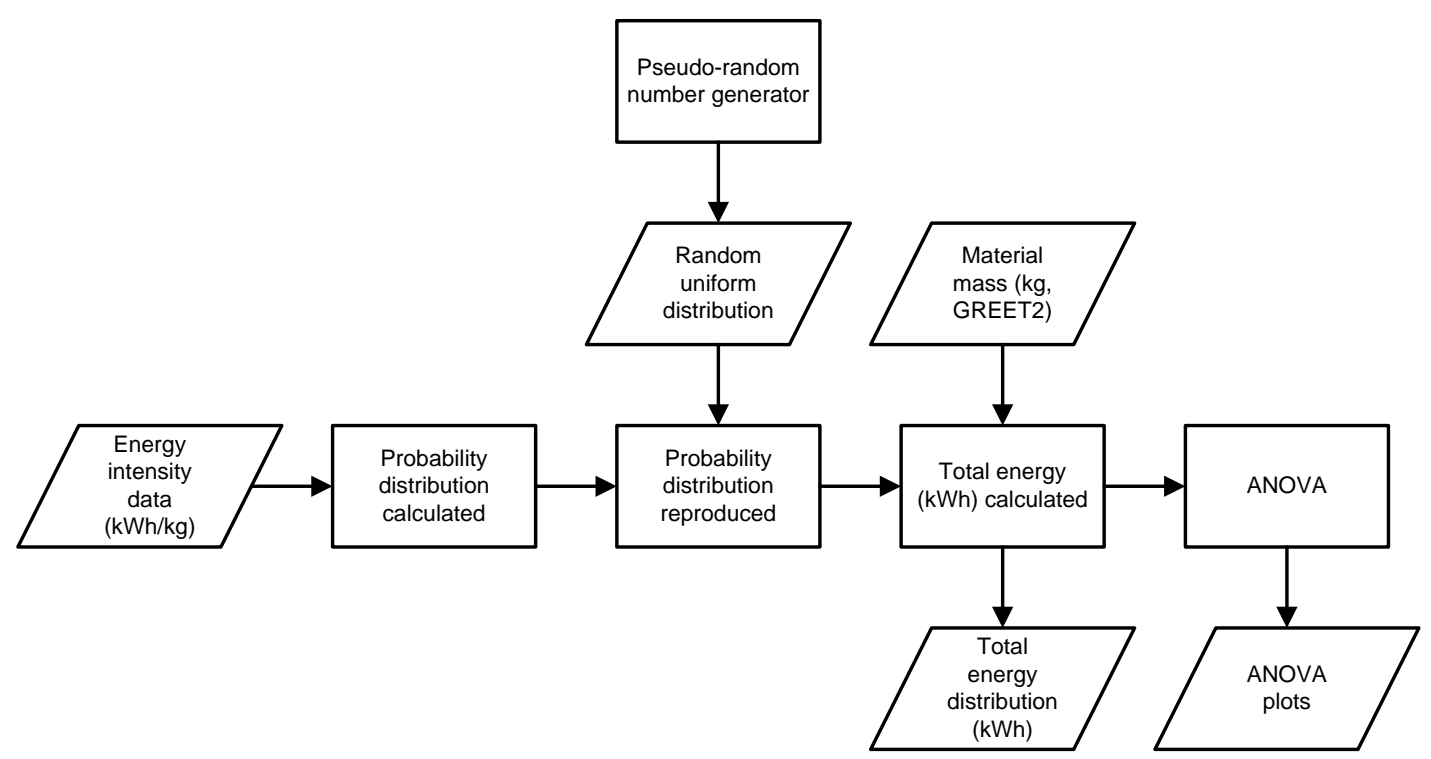

Figure 3.1 An overview of Chapter 3 and the Monte Carlo simulation.

\subsubsection{Monte Carlo Simulations}

Monte Carlo simulations are an established method for studying uncertainty and variability in LCA (Hertwich et al., 2000; Björkland, 2002; Heijungs and Frischnecht, 2005). Indeed, this methodology has been used before in some practical studies (Kennedy et al., 1996; Huijbregts et al., 2003), as well as the Monte Carlo variant known as Latin Hypercube sampling (Huijbregts, 1998b). In this instance, variability may be understood to be the product of inherent variation observed in the real world (Huijbregts, 1998a). Stated another way, this variability may represent the true diversity or heterogeneity of a properly representative population (U.S. Environmental Protection Agency, 1997). Uncertainty may consist of data arising from measurement inaccuracy or a lack of data, whereby lack of data may be understood to consist of gaps in data or unrepresentative data (Huijbregts et al., 2001). However, in this case, Huijbregts et al. (2001) mean data that is missing between inter-process stages which may be dealt with, for example, using a mass balance approach to fill these gaps. However, this does not have any 
Table 3.1 An exhaustive list of background effects and their relevance to this study.

\begin{tabular}{|c|c|}
\hline Background Process Cause & Relevance to this Study \\
\hline \multicolumn{2}{|l|}{ Hendrickson et al., 1997} \\
\hline $\begin{array}{l}\text { Comprehensiveness of process inclusion } \\
\text { (i.e., system boundaries) }\end{array}$ & $\begin{array}{l}\text { System boundaries (e.g., transportation) are included in the } \\
\text { data utilized. }\end{array}$ \\
\hline $\begin{array}{l}\text { Differences in the energy mix of each } \\
\text { country }\end{array}$ & Are included and have relevance to this study. \\
\hline Differences in energy efficiency & Are included and have relevance. \\
\hline Comprehensiveness of impact inclusion & This relates to LCIA and has no relevance here. \\
\hline $\begin{array}{l}\text { Differences in end of pipe treatment due } \\
\text { to distinct regulatory requirements }\end{array}$ & Not relevant. This refers to emissions. \\
\hline Temporal differences & Are relevant. \\
\hline Differences in definitions of outputs & $\begin{array}{l}\text { This has relevance to this study. However, this was } \\
\text { accounted for by eliminating secondary industrial processes } \\
\text { (e.g., galvanising, pickling, extrusion, etc.) and focusing on } \\
\text { primary process energy. }\end{array}$ \\
\hline $\begin{array}{l}\text { Comprehensiveness of measurement } \\
\text { and error in reporting }\end{array}$ & $\begin{array}{l}\text { This pertains to differences arising from whether a process- } \\
\text { based or economic input-output format for reporting was } \\
\text { used. Hence, this has no application to this study since only } \\
\text { a process-based format data was used. }\end{array}$ \\
\hline Level of aggregation (of data) & $\begin{array}{l}\text { This relates to differences in definitions of outputs arising } \\
\text { from whether process-based or economic input-output } \\
\text { methods were used and has no relevance here. }\end{array}$ \\
\hline \multicolumn{2}{|l|}{ Peereboom et al., 1999} \\
\hline $\begin{array}{l}\text { The geographical, temporal, and } \\
\text { technological representativeness }\end{array}$ & $\begin{array}{l}\text { All of these are relevant to this study and are part of the } \\
\text { study's findings. }\end{array}$ \\
\hline Level of categorization of data & $\begin{array}{l}\text { This pertains to the level of specificity in data. For example, } \\
\text { data on the energy intensity of steel may mean many } \\
\text { different things since there are many different types of steel. } \\
\text { Again, this was compensated for by the focus on primary } \\
\text { production and not finished products. }\end{array}$ \\
\hline Allocation method & $\begin{array}{l}\text { This may have some influence on the results; however, it is } \\
\text { assumed that this has been addressed by the data compiler. }\end{array}$ \\
\hline $\begin{array}{l}\text { Each inventory data set contains data } \\
\text { from different data sources }\end{array}$ & $\begin{array}{l}\text { LCA studies may use data from other studies. Every effort } \\
\text { was made to track down the original data source to prevent } \\
\text { duplication. }\end{array}$ \\
\hline $\begin{array}{l}\text { Differences exist in level of aggregation } \\
\text { of processes }\end{array}$ & $\begin{array}{l}\text { In this instance, this means whether the data has been } \\
\text { aggregated over many individual unit processes. Again, it is } \\
\text { assumed that the original data compiler has accounted for } \\
\text { this. }\end{array}$ \\
\hline Lack of metadata & Not relevant here. Only discreet data points were used. \\
\hline Lack of metainformation & Not relevant here. \\
\hline \multicolumn{2}{|l|}{ Takano et al., 2014} \\
\hline Number of construction material data & $\begin{array}{l}\text { This refers to the problems posed by missing data which } \\
\text { must to be compensated for in the final results of an LCA. } \\
\text { For example, if data on product A was missing, substitute } \\
\text { product B with similar characteristics would have to be used } \\
\text { instead. Consequently, this is not relevant here. }\end{array}$ \\
\hline
\end{tabular}


relevance to this study. Therefore, in this study, uncertainty refers strictly to missing data, as will be illustrated and further elaborated below.

Maurice et al. (2000) outline the procedure for a Monte Carlo simulation. A probability distribution for the input data must be determined such that the distributions of the output data are calculated using the Monte Carlo simulation. The latter involves randomly sampling the probability distribution such that the input data's probability distribution is reproduced. In this study, Monte Carlo simulations were built in MATLAB (versions 2013b through 2014a) and utilized $10^{8}$ iterations each to generate the randomized data set of estimators for finding the energy totals in Table 3.3. That is, 10,000 simulations were run, each with 10,000 iterations (point estimations) for each simulation. Past prohibitions of this type of study were based on the fact that data was not readily available for these kinds of assessments. In turn, this necessitated making assumptions regarding the type of probability distribution utilized. For example, Kennedy et al. (1996) utilized a beta probability distribution and Raynolds et al. (1999) used a normal probability distribution in the absence in sufficient data to generate an actual probability distribution. In the case of this study, probability distributions were assembled using available data from a number of literature sources (see below). However, it should be mentioned here that there is an underlying assumption regarding making inferences from distributions produced from a low number of data points. Namely, when there are a low number of data points, the measured variance distribution enters into territory which can be identified as uncertainty analysis.

Some discussion must be made here regarding the phenomenon of error propagation in Monte Carlo simulations. A standard definition of error propagation in simulations is given by Ogilvie (1984) where for $m$ inputs $\left(I_{1}, I_{2}, \ldots, I_{i}, \ldots, I_{m}\right)$ and $n$ outputs $\left(O_{1}, O_{2}, \ldots, O_{j}, \ldots, O_{n}\right)$, small variations or deviations $\delta I_{i}$ in individual $I_{i}$ values will result in corresponding effects $\delta O_{\mathrm{j}}$ on $O_{j}$ output parameters such that:

$$
\delta O_{j}=\sum_{i=1}^{m} \delta I_{i} \frac{\partial O_{j}\left(I_{1}, I_{2}, \ldots, I_{m}\right)}{\partial I_{i}}
$$

Here, the calculation of the $m+1$ derivatives $\partial O_{j} / \partial I_{i}$ are given as the following individual terms: 


$$
\begin{aligned}
& O\left(I_{1}, I_{2}, \ldots \ldots, I_{m}\right) \\
& O\left(I_{1}+\varepsilon_{1}, I_{2}, \ldots ., I_{m}\right) \\
& O\left(I_{1}, I_{2}+\varepsilon_{2}, \ldots, I_{m}\right) \\
& O\left(I_{I}, I_{2}, \ldots, I_{m}+\varepsilon_{m}\right)
\end{aligned}
$$

where $\varepsilon_{i}=\delta I_{i} / I_{i}$. MATLAB utilizes a Mersenne Twister pseudo-random number generator (PRNG) known as MT19937. Tests conducted on MT19937 have shown consistent results such that the standard error of this PRNG does not exceed their error bars (Hongo et al., 2010; Hongo and Maezono, 2011). Consequently, the MT19937 is deemed to be an unbiased estimator (Ibid.). This means that there is no error in the form of bias originating from the PRNG. This is evident from the fact that the Mersenne Twister has a very large period at $2^{19937}-1$ and a 623-dimensional equidistribution up to 32-bit accuracy (Matsumoto and Hishimura, 1998). Hence, the outputs are free from long-term correlations. Also, although 95\% confidence intervals were calculated for the inputs in Table 3.2, there are no variances of these data sets in the model during the simulation (e.g., as would be seen in bootstrapping methods). These $95 \%$ confidence intervals were used merely to characterize the data sets of energy intensity for individual materials. Furthermore, the same function (ksdensity) is used to calculate the probability distributions (e.g., Figure 3.5 and 3.6) ${ }^{1}$. This study also uses a very large number of iterations $\left(10^{8}\right)$ which is conducive of robust results. This is evidenced by the closeness of the median to estimation of the mean, as well as the very small standard errors $(S E)$ as seen in Table 3.3. Because of these factors, error propagation as defined by Ogilvie (1984) does not have pertinent effects on the findings of their study.

Additional reasons why MATLAB software was chosen over other professional LCA software platforms such as GaBi or SimaPro are important. As shown in Table 3.3 below, SimaPro's literature indicates that their Monte Carlo simulations only allow for four types of probability distribution: normal, lognormal, range (constant), and triangular (Goedkoop et al, 2013). Hence, SimaPro does not allow for the construction of individual probability distributions from raw data.

\footnotetext{
${ }^{1}$ See Appendix A for all the probability distributions for individual materials of manufacture.
} 
Table 3.2 The raw materials of manufacture directly from GREET2 model and their corresponding weights in kilograms.

\begin{tabular}{|c|c|c|c|c|c|c|c|c|c|c|}
\hline & \multirow[b]{2}{*}{ Data range } & \multirow{2}{*}{$\begin{array}{l}\text { Mean } \\
(\mathrm{kWh} / \mathrm{kg})\end{array}$} & \multirow{2}{*}{$\begin{array}{l}\text { Std dev } \\
(\mathrm{kWh} / \mathrm{kg})\end{array}$} & \multirow{2}{*}{$\begin{array}{l}95 \% \text { Conf } \\
\text { Interval }\end{array}$} & \multicolumn{3}{|c|}{ Regular (kg) } & \multicolumn{3}{|c|}{ Lightweight (kg) } \\
\hline & & & & & Sedan & SUV & PUT & Sedan & SUV & PUT \\
\hline Cast iron & 1973-2012 & 7.51 & 2.96 & 1.03 & 142.7 & 178.2 & 221.8 & 27.8 & 38.1 & 46.2 \\
\hline Copper & 1968-2012 & 12.22 & 7.49 & 2.60 & 24.9 & 24.6 & 27.1 & 23.9 & 10.7 & 25.4 \\
\hline Lead & 1973-2012 & 10.02 & 8.06 & 2.79 & 16.3 & 16.3 & 16.3 & 7.3 & 7.3 & 7.3 \\
\hline Steel & 1960-2011 & 5.34 & 4.10 & 1.42 & 600.1 & 725.5 & 844.6 & 180.2 & 211.5 & 275.2 \\
\hline Recycled steel & 1981-2009 & 5.07 & 5.51 & 1.91 & 215.3 & 260.2 & 303 & 64.6 & 75.9 & 98.7 \\
\hline Stainless steel & 2003-2011 & 13.19 & 9.12 & 3.16 & & & & 8.5 & 10.1 & 9.7 \\
\hline Magnesium & 1983-2012 & 87.82 & 26.65 & 9.23 & & & & 3.1 & 2.7 & 2.7 \\
\hline Aluminum & 1968-2012 & 23.00 & 17.41 & 6.03 & 28.8 & 29.5 & 40.4 & 63.0 & 55.1 & 68.9 \\
\hline Recycled aluminum & 1983-2009 & 13.16 & 12.86 & 4.46 & 54.3 & 75.0 & 88.9 & 101.5 & 168.3 & 271.1 \\
\hline HDPE (high density polyethylene) & 1973-2012 & 23.19 & 6.68 & 2.31 & 30.2 & 31.8 & 33.7 & 22.2 & 27.2 & 26.9 \\
\hline PP (polypropylene) & 1973-2011 & 23.76 & 7.66 & 2.65 & 71.3 & 76.3 & 81.1 & 53.3 & 65.5 & 64.6 \\
\hline PET (polyethylene terephthalate) & 1979-2012 & 22.77 & 6.79 & 2.35 & 42.4 & 44.6 & 47.3 & 31.1 & 38.2 & 37.7 \\
\hline CFRP (carbon fibre reinforced plastic) & $1990-2013$ & 60.63 & 11.95 & 4.14 & & & & 116.6 & 151.4 & 139.2 \\
\hline GFRP (glass fibre reinforced plastic) & 1983-2012 & 22.6 & 7.74 & 2.68 & & & & 13.9 & 16.9 & 21.1 \\
\hline Rubber & 1968-2012 & 5.94 & 7.69 & 2.66 & 30.1 & 31.6 & 48.7 & 30.1 & 34.4 & 38.5 \\
\hline Glass & 1968-2012 & 5.11 & 4.53 & 1.57 & 38.0 & 49.2 & 47.7 & 23.2 & 36.3 & 31.8 \\
\hline Paint & 1993-2012 & 19.62 & 21.31 & 7.38 & 11.8 & 11.8 & 11.8 & 5.9 & 5.9 & 5.9 \\
\hline & & & & & 1306.2 & 1554.6 & 1812.4 & 776.17 & 955.5 & 1170.9 \\
\hline
\end{tabular}

Table 3.3 The types of fixed distributions used by SimaPro to model uncertainty (adapted from Goedkoop et al., 2013).

\begin{tabular}{|l|l|l|}
\hline Distribution & Data Needed & Graphical Presentation \\
\hline $\begin{array}{l}\text { Range } \\
\text { (Uniform) }\end{array}$ & Min and max value & \\
\hline Triangular & Min and max value plus best guess value & \\
\hline $\begin{array}{l}\text { Normal } \\
\text { distribution }\end{array}$ & Standard deviation and best guess value & \\
\hline $\begin{array}{l}\text { Lognormal } \\
\text { distribution }\end{array}$ & Standard deviation and best guess value & \\
\hline
\end{tabular}

The reason for this is that SimaPro uses these distributions to model uncertainty, and not variance. SimaPro also does not have the ability to test for non-normal distributions by measurements of skewness or kurtosis. As well, there is no public literature available on the pseudo-random number generator (PNRG) used by SimaPro; hence, an analysis of the PNRG's error propagation (bias) is impossible. Many of the available software platforms in LCA were not utilized in this study owing to the limited ability to assess their functionality from available 
literature and demo software versions. For example, very little public information exists of the GaBi platform's Monte Carlo simulations.

\subsubsection{Data collection and utilization}

After reviewing several different LCA software platforms for this research (such as SimaPro, $\mathrm{GaBi}$, and Umberto), it was decided that the GREET2 model would be utilized. Greenhouse gases, Regulatory Emissions, and Energy use in Transportation (GREET) is a complete, standalone LCA by the Argonne National Laboratory, United States Department of Energy. It is a freely available LCA in Microsoft Excel format that can be modified to test specific design issues with the aim of informing comparative assertions. GREET1 comprises the fuel cycle of automobiles from raw material acquisition, refining and, finally, usage. Similarly, GREET2 comprises the manufacture and usage of an automobile from raw material acquisition, manufacture, usage and disposal. For the purposes of this study, only the raw material extraction and manufacture phases of GREET2 were investigated, while the vehicle use and final disposal phases were disregarded. This promotes the furtherance of comparative assertions. Further information on specifics related to GREET2 modelling can be found in Hawkins et al. (2013).

Energy intensity $(\mathrm{kWh} / \mathrm{kg})$ of materials for manufacture was chosen as the metric for this study as there were a number of benefits behind their usage. First, unlike other monetary-based analyses of time-series data (e.g., economic input-output studies), the effects of inflation and valuation do not obscure the results. There are also benefits to using energy intensity as an impact category. Other common impact categories in LCA are either dependent on the stock of available materials/resources or are dose dependent (see Pelletier et al., 2007). Hence, as a physical metric, energy intensity is subject to real-world variability which is not affected by erroneous background processes (see Table 3.1). The last benefit was that there were known databases of energy intensity of materials of manufacture extending back in time.

The 17 individual raw materials GREET2 used for manufacture and their corresponding mass by vehicle type are listed in Table 3.2. It will be noted that the values for lead and paint are consistent. In the case of lead, this is owing to the use of a standard battery. With the latter, the 
same value is used because GREET2 only specified the weight of paint used in a sedan. Energy intensity of the raw materials consisted of the extraction of raw materials onwards to primary or secondary manufacture (cradle-to-gate). With few exceptions, most of the data on energy intensity was gathered from the following types of sources (full references in Appendix A):

- International databases (World Steel, International Aluminium Association, etc.)

- Peer-reviewed papers

- Congresses, proceedings and technical papers

- Government reports

- CSR (Corporate Social Responsibility) reports

- Life Cycle Assessment findings

- Books (selection and manufacture of materials) and book chapters

For the preliminary findings presented here, approximately 323 documents served as the source for all data utilized. The data search and collection was exhaustive with the aim of utilizing as much data as possible. The data collected here exceeds any publicly available database on energy intensity of production, including the Inventory of Carbon and Energy (ICE) from the University of Bath (UK), the Interuniversity Research Centre for the Life Cycle of Products, Processes and Services (CIRAIG) of Canada, SPINE (Sustainable Product Information Network for the Environment) of Sweden, and the Centre for Building Performance Research of New Zealand (to cite a few). For example, the ICE database has 247 documents but has roughly 189 different materials and products, many of which are not used in this study: asbestos, carpet, ceramics, clay, concrete, fly ash, plaster, stone, straw, timber, vermiculite, and so forth (Hammond and Jones, 2008). For this study, it follows that there is intrinsic value in collecting such a large body of data as this can serve as the basis for more accurate and conservative estimations of variability.

After data was collected, consolidation ensued by reviewing the source documents. Data consolidation was necessary due to the fact that some data from the fields of Building Science and Architecture had to be discarded as the units of energy intensity were expressed in terms of the area of the building (e.g., $\mathrm{J} / \mathrm{m}^{2}$ ) and, in some rare cases, in units of volume (e.g., $\mathrm{J} / \mathrm{m}^{3}$ ). The 
data used in this study was geographically specific to varying degrees. Some sources provided the exact location of a smelting operation while others merely mentioned the country of origin or a general area (e.g., the EU). Hence, this is why it was decided to frame the spatiality element of this study to the continental level. The data sources provided sufficient information so that they could be classified either by primary or secondary processes. By and large, secondary process data were capable of being classified according to process method such as cold or hot rolled, extruded, drawn, galvanized, etc., or product such as wire, plate, ingot, etc. Unfortunately, confidence intervals on the source data of energy intensity were very rare and this made their assessment from the input perspective impossible. Nevertheless, an assessment was made of the data on energy intensity in Table 3.2. However, it should be noted that the calculated means, standard deviations, and 95\% confidence intervals in Table 3.2 are based on the size of the pool of collected data (i.e., number of data points). Hence, these calculations are meant to characterize the pool of collected data, not to assess their representativeness. With this in mind, it can be seen that although the distribution (standard deviation) of energy intensity around some materials (recycled aluminum and steel) is large, the 95\% confidence intervals for most of the materials indicates a good representation for repeated multiple samples. Other materials such as magnesium, aluminum, recycled aluminum, and paint see higher confidence intervals due to either small sample size or large variance.

Historic data on the energy intensity of individual materials $i$ was imported from Microsoft Excel according to various filters set up in the MATLAB Monte Carlo simulation program (e.g., time frame, country, production process, etc.). An example of raw historic energy intensity $(\mathrm{kWh} / \mathrm{kg})$ for a single material is illustrated in Figure 3.2. The figure shows the energy intensity of steel as a function of time. Here, a first order least square linear regression was performed as higher order regressions resulted in poorer fits. The regression analysis line can be seen indicating the relationship between energy intensity and time. This regression analysis indicates a downward trend in energy intensity. However, this regression line is used purely for the purpose of illustration. As explained below, the determination of a probability distribution was based on individual data points. Figure 3.3 displays the energy intensity of aluminum manufacture. Uncertainty can be seen in the plot by sparse or missing data between the years 1970 and 1990 even though, once again, the regression analysis line indicates a general downward trend of 
energy intensity. Also of concern with regard to the uncertainty are the outliers as shown in Figure 3.2.

Utilizing MATLAB's kernel smoothing function estimate (ksdensity) allowed for this historical data to be converted into a probability distribution (e.g., see Figure 3.5). Subsequently, using this probability distribution, a cumulative probability distribution was constructed (e.g., see Figure 3.6) by which the randomized set could be fit to generate an energy per mass $(\mathrm{kWh} / \mathrm{kg})$ distribution $d_{i}$ for each individual material. Then, using known masses $m_{i}$ specified by GREET2 for each corresponding individual material $i$ (steel, aluminum, copper, etc.), the total energy per vehicle was calculated from the individual material distributions. Thus, the total energy per vehicle $(E)$ equation would be:

$$
E=\sum_{i=1}^{N} m_{i} d_{i}
$$

An energy total was calculated by Monte Carlo simulation for each different vehicle type (see Table 3.4). The total number of materials $N$ under study here is 17 individual materials (see Table 3.2). Different vehicle types given by GREET2 were a sedan, sport-utility vehicle (SUV), and pick-up truck (PUT). GREET2 also models lightweight vehicles which use less traditional materials such as steel and cast iron and more alternative materials such as aluminum, carbon fibre reinforced plastic (CFRP), glass fibre reinforced plastic (GFRP), and magnesium. Naturally, the underlying motive for lightweight vehicles is the promotion of greater fuel efficiency. When traditional materials such as steel and cast iron are substituted for more unconventional materials such as aluminum, carbon fibre reinforced plastic (CFRP), glass fibre reinforced plastic (GFRP) and magnesium, the total mass of the sedan vehicle is reduced by $526 \mathrm{~kg}$. The purpose of performing Monte Carlo simulations on these types of vehicles is to provide comparative scenarios to other functional units constructed of different materials. In doing so, this makes possible the comparison of the individual contributions of each material to examine whether the ICEV sedan results are anomalous. These different vehicle configurations became the basis for further comparative assertions (see below). 
One basis for making comparative assertions was that the variance of the full time scale be compared to a smaller time frame with the assumption that the latter would see significant reductions in both variance and total average energy. As a result of this, the choice of the smaller five year time interval was based on two factors:

1) Data quality indicators - also known as a pedigree matrix — were developed by Weidema and Wesnaes (1996) to indicate five time intervals for consideration according to their pedigree score (in brackets): less than 3 years (1); less than 6 years (2); less than 10 years (3); less than 15 years (4); and more than 15 years or date unknown (5). Weidema and Wesnaes (1996) indicate that a level 2 (less than 6 years) temporal correlation was less likely to be susceptible to large advances in technological progress. Furthermore, with the exception of industrial sectors such as electronics and the pulp and paper industry, Weidema (1998) indicated that a temporal correlation score of 2 (less than 6 years) was not indicative of rapid industrial technological development.

2) The minimum number of data points necessary for a kernel density estimator for a univariate data set. Silverman (1998) proposed that, for a single point kernel density estimate (i.e., at $x$ ), the mean square error $(M S E)$ be given as follows:

$$
M S E=[\hat{f}(x)-f(x)]^{2}
$$

where $\hat{f}$ is the density estimator and $f$ is the true density. Silverman (1998) then goes on to state that a desirable objective would be to keep the relative mean square error (RMSE) less than 0.1 at a single point (0). Stated formally yields the following expression:

$$
R M S E=\frac{[\hat{f}(\mathbf{0})-f(\mathbf{0})]^{2}}{f(\mathbf{0})^{2}}<0.1
$$




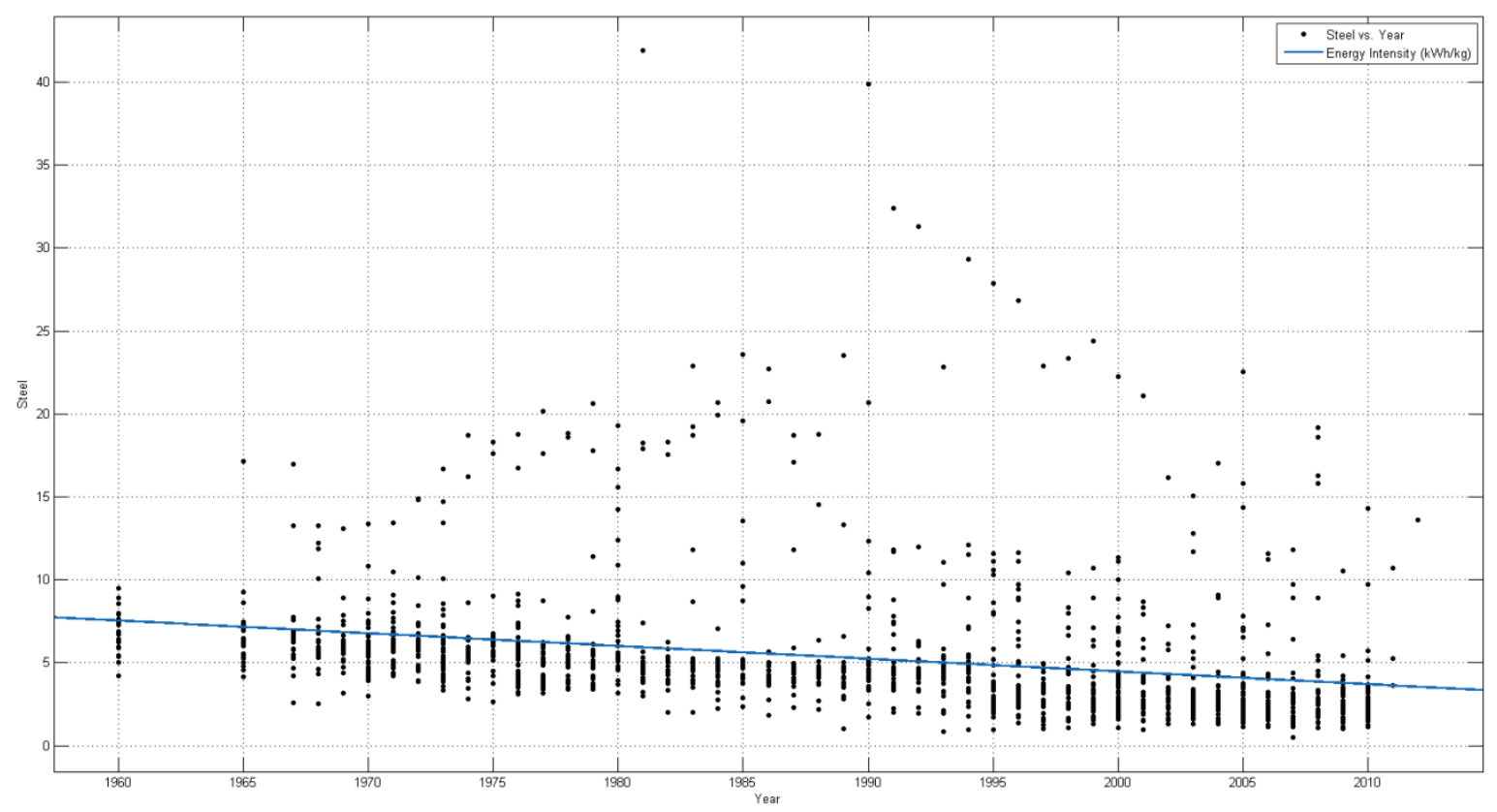

Figure 3.2 Raw data of historic energy intensity $(\mathrm{kWh} / \mathrm{kg})$ for primary steel.

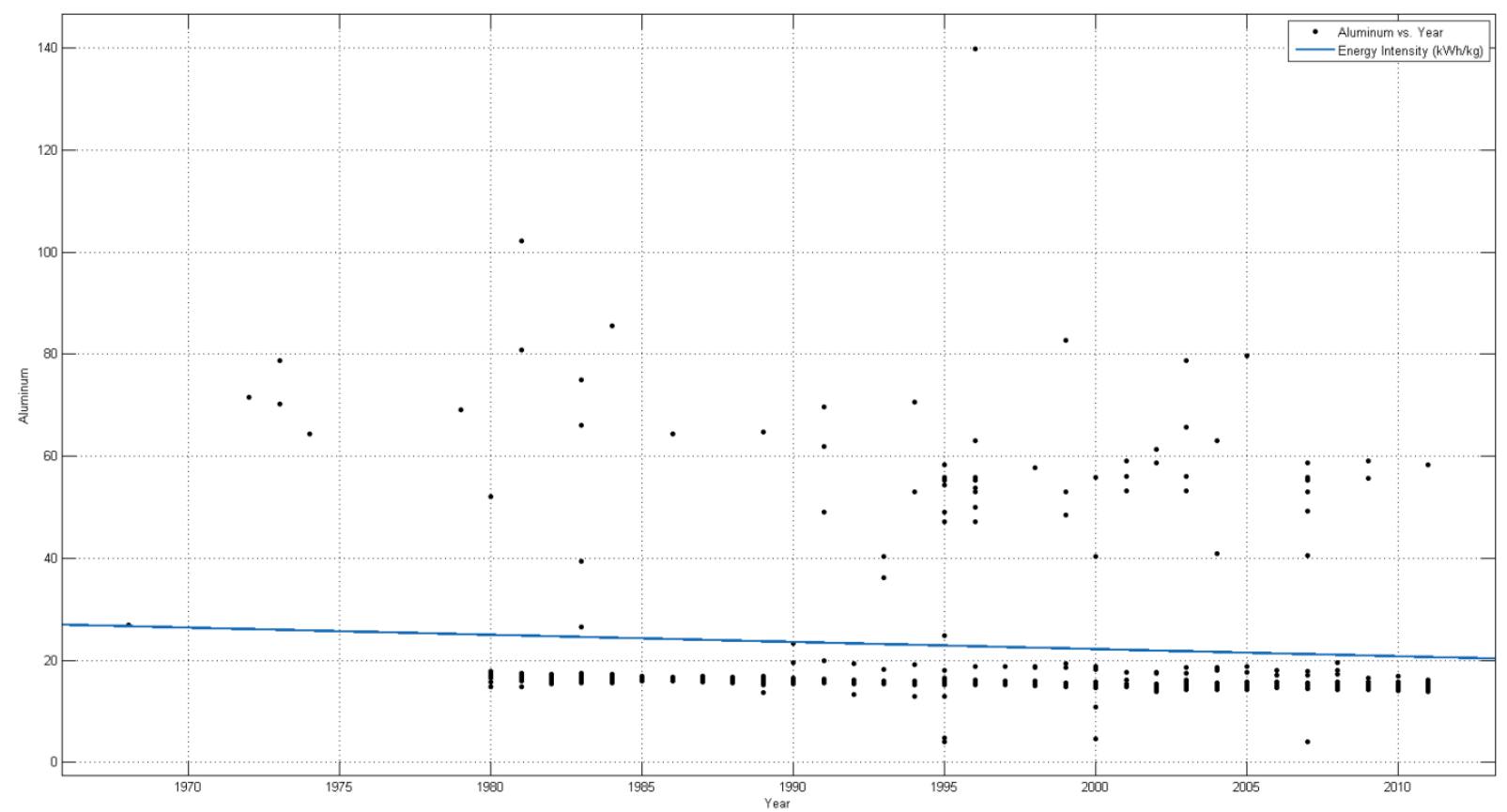

Figure 3.3 Historical raw data of energy intensity $(\mathrm{kWh} / \mathrm{kg})$ of primary aluminum. 
In accordance with this, Silverman calculated that at least four data points (sample size) would be necessary to meet this criterion for a univariate data set. For the majority of materials in this study, the sample size for a five year time interval exceeded the minimum sample size. However, for materials such as PET, HDPE and cast iron, the sample size was equal to the minimum criterion of a five year interval. Hence, this factored into the choice to model the variance for the smaller time frame at five years.

\subsubsection{Coefficient of variance (CV), standard error (SE), and ANOVA}

The main benefit behind the usage of the coefficient of variation $(C V)$ is that the standard deviation increases or decreases in proportion to increases or decreases in the mean value of a distribution (Reed et al., 2002). Hence, this has the effect of reducing the effect of changes in the mean such that variability can be assessed in comparative terms (Ibid.). The formula for $C V$ is:

$$
C V=\frac{\sigma}{\bar{x}}
$$

where $\sigma$ is the standard deviation and $\bar{x}$ is the mean. Comparative assertions regarding $C V$ values will follow methods used in Wang and Shen (2013), Escobar et al. (2014), as well as Chen and Corson (2014). As such, variation in CV values will be measured in relative percentage changes to the five year time frame (2007-2012) since the full time frame includes the five year time frame. This is further articulated in the Results chapter (3.3).

Similarly, standard error (SE) will be calculated using

$$
S E=\frac{\sigma}{\sqrt{n}}
$$

where $\sigma$ is the standard deviation and $n$ is the number of iterations (measurements), which is $10^{8}$ for each vehicle type. Consequently, the $95 \%$ confidence intervals can be calculated from these measurements of $S E$ by multiplying $S E$ by 1.96 (under the assumption the data are normally distributed). 
Lastly, a one-way analysis of variance (ANOVA) was utilized in order to detect which of the materials of manufacture was contributing the greatest to the measured variance. The ANOVA was done using MATLAB. Box plots of the ANOVA are given in Figures 3.7 and 3.8. In the box plot the centre line of each box represents the median, while the vertical edges of the box are the $25^{\text {th }}$ and $75^{\text {th }}$ percentiles. The outer whiskers represent (+/-) 2.7 standard deviations or 99.3 percent coverage for a normal distribution. Extreme outliers beyond the whiskers are represented by individual data points. Alongside these box plots, an F-test was conducted of the Monte Carlo distributions in order to make comparisons of the effects of changes in time frame. Consequently, the F-ratio was calculated by dividing the treatment mean square (MSTR) by the mean square error $(M S E)$ :

$$
F=\frac{M S T R}{M S E}
$$

where

$$
M S T R=\frac{S S T R}{k-1}
$$

and

$$
M S E=\frac{S S E}{n-k}
$$

The sum of the squares for treatment (SSTR) and the sum of the squares for error $(S S E)$ are calculated using

$$
S S T R=\sum n_{j}\left(\bar{x}_{j}-\bar{x}\right)^{2}
$$

and

$$
S S E=\sum\left(n_{j}-1\right) s_{j}^{2}
$$

where $k=$ number of populations

$n=$ total number of observations

$n_{j}=$ size of sample from population $j$

$\bar{x}=$ mean of all $\mathrm{n}$ observations

$\bar{x}_{j}=$ mean of sample from population $j$

$s_{j}^{2}=$ variance of sample from population $j$ 
Lastly, the $\mathrm{F}$ critical values $\left(F_{c r i t}\right)$ were calculated using a specified significance level (1- $\left.\alpha\right)$ on simple Excel spreadsheet.

\subsubsection{Skewness and Kurtosis}

As this chapter examines the potential distorting effects of the raw data (energy intensity), a log transformation method (base 10) is utilized in order to test whether the original distribution is non-normal. Log transformation is a simple method of converting normal in situ data into a logarithmic distribution for the purposes of bringing about greater visual clarity and to see whether the original data displays a log-normal distribution (National Institute of Science and Technology, 2012). If the original data proves to be non-normal, this would have implications in utilizing variance to analyze temporality and spatiality due to aged data. Skewness can be understood as the overall lack of symmetry of a distribution while kurtosis is the flatness of a distribution (Ibid.). It is expected that when normal data is log transformed, that there will be an overall increase in skewness (the distribution will become more symmetrical) and decrease in kurtosis (the distribution will become more flat). The typical formulas for skewness and kurtosis are given as:

$$
\begin{aligned}
& \text { skewness }=\frac{\sum_{i=1}^{n}\left(x_{i}-\bar{x}\right)^{3} / n}{\sigma^{3}} \\
& \text { kurtosis }=\frac{\sum_{i=1}^{n}\left(x_{i}-\bar{x}\right)^{4} / n}{\sigma^{4}}
\end{aligned}
$$

where, as with above, $\bar{x}$ is the mean and $\sigma$ the standard deviation. Furthermore, $n$ denotes the number of measurements in this distribution, which in this case is ten thousand.

\subsubsection{Limitations}

Limitations of the study include a lack of data from Asia for most of the materials under study. Sufficient data from Japan, China, Korea, etc., can be found for steel and aluminum, but is highly 
lacking outside of these two materials. Other studies on energy intensity which were not English or German language based were excluded. Furthermore, as there are a multitude of final products and processes which secondary processes could bring about, the data used was restricted to primary process energy for steel, aluminum and copper (except in instances where recycled material is substituted). Nevertheless, as comparative assertions are the main focus of this study, this was deemed a necessary trade-off. Also, recycled materials used in this study were limited to data where $100 \%$ recycled matter was used instead of some pre-formatted combination or mixture (e.g., $70 \%$ virgin, $30 \%$ recycled). Temporal comparisons of magnesium, stainless steel, CFRP, and GFRP could not be made owing to limited data. Nevertheless, their energy contributions have been included in the total energy calculations for the lightweight vehicles.

Only internal combustion engine vehicles (ICEV) are the subject of this study. Other types of power trains will be the subject of chapter 4 . Spatiality comparisons are limited to regular sedans and to general geographies such as North America, Europe, and Oceania (Australia and New Zealand). Consequently, the areas they cover are vast and this must be kept in mind when examining the results. Spatial calculations had to exclude cast iron, recycled steel, and recycled aluminum as there was not sufficient data to enable analysis. Also, not all data used in this study spans the years from 1960 to 2012. Only primary steel has data that stretches that far back in time. Most of the other data ranges are variegated (see Table 3.2). This has important consequences when examining the total energy results of temporality due to age of data.

\subsection{Results and Discussion}

\subsubsection{Monte Carlo simulations and analysis of variance (temporality)}

Tables 3.4 and 3.5 outline the results of the Monte Carlo simulations for total energy of an internal combustion engine vehicle in three different vehicle classes. Table 3.4 can be broken down into vertical and horizontal axes. The horizontal axis denotes vehicles composed of regular materials and lightweight vehicles manufactured with substitute materials (see Table 3.2). Hence, it can be seen that the lightweight vehicles have a smaller mean total energy ( $\mathrm{kWh})$ 
as compared to regular vehicles. The vertical axis contains vehicles with the full time scale (see Table 3.2) and the five year time scale (2007-2012). It will be noted that individual material

Table 3.4 The total energy $(E)$ per vehicle in $\mathrm{kWh}$, standard deviation $(S D)$, standard error $(S E)$ and coefficient of variation $(C V)$ using regular and lightweight materials.

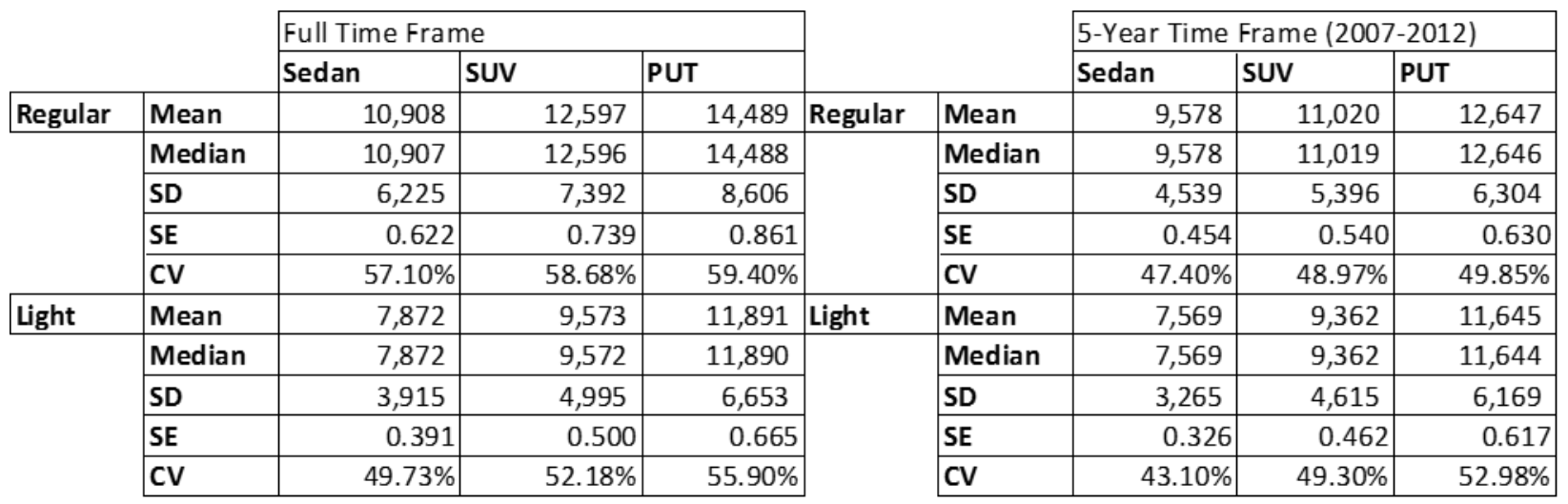

Table 3.5 The total energy $(E)$ per vehicle (sedan) in $\mathrm{kWh}$, standard deviation $(S D)$, standard error $(S E)$ and coefficient of variation $(C V)$ according to geographic location.

\begin{tabular}{|l|r|r|r|}
\cline { 2 - 4 } \multicolumn{1}{c|}{} & \multicolumn{1}{l|}{ North Am } & \multicolumn{1}{l|}{ Europe } & Oceania \\
\hline Mean & 11,047 & 10,347 & 10,463 \\
\hline Median & 11,047 & 10,346 & 10,463 \\
\hline SD & 5,868 & 5,874 & 6,081 \\
\hline SE & 0.587 & 0.587 & 0.608 \\
\hline CV & 0.531 & 0.568 & 0.581 \\
\hline
\end{tabular}

time scales in the full time scale scenario are not the same. For example, steel data ranges from 1960 to 2011 while cast iron data ranges from 1973 to 2012 . The reason for this is the desire to see what the full range of variance in each individual material was and compare that to the five year time frame (2007-2012). (This will figure in the recommendations for future analysis below.) It will be noted that the heavier the vehicle, the higher the mean total energy and the corresponding standard deviation $(S D)$, standard error $(S E)$, and coefficient of variation $(C V)$. This is owing to the use of more amounts of material in the SUVs and PUTs: more cast iron, steel, recycled steel, aluminum, recycled aluminum, plastics, as well as rubber and glass. Furthermore, it may be seen that all of these measurements decrease in the five year time frame. 
For the purposes of comparison, the total energy of ICEV sedan vehicles from cradle-to-gate for the materials production phase is presented in Table 3.6 below. For the most part, these studies were compiled using industry-sourced data, while Schweimer and Levin (2000) used GaBi

Table 3.6 Comparison of total energy of vehicle (cradle-to-gate) from other studies.

\begin{tabular}{|l|l|l|l|l|}
\hline Study & $\begin{array}{l}\text { Year of } \\
\text { Vehicle }\end{array}$ & $\begin{array}{l}\text { Vehicle Weight } \\
(\mathbf{k g})\end{array}$ & Processes & $\begin{array}{l}\text { Total Energy } \\
(\mathbf{k W h})\end{array}$ \\
\hline $\begin{array}{l}\text { Barry and Fels } \\
(1971)\end{array}$ & 1967 & 1,555 & Primary & 22,823 \\
\hline $\begin{array}{l}\text { Stodosky et al. } \\
(1995)\end{array}$ & 1994 & 1,314 & Primary & 20,240 \\
\hline $\begin{array}{l}\text { Sullivan and Hu } \\
(1995)\end{array}$ & 1995 & 1,157 & Primary & $18,609^{*}$ \\
\hline Schuckert (1996) & 1996 & 1,160 & Primary & $18,611^{*}$ \\
\hline $\begin{array}{l}\text { Dhingra et al. } \\
(1999)\end{array}$ & 1994 & 1,474 & Primary & 9,322 \\
\hline $\begin{array}{l}\text { Scheweirmer and } \\
\text { Levin (2000) }\end{array}$ & 1999 & $1,059(1.4 \mathrm{~L}$ petrol) & Primary & 13,333 \\
\hline $\begin{array}{l}\text { Scheweirmer and } \\
\text { Levin (2000) }\end{array}$ & 1999 & $1,181(1.9 \mathrm{~L}$ diesel) & Primary & 14,222 \\
\hline $\begin{array}{l}\text { Schexnayder et al. } \\
(2001)\end{array}$ & 1994 & 1,474 & Primary & 9,322 \\
\hline $\begin{array}{l}\text { Zamel and Li } \\
(2006)\end{array}$ & 1999 & 1,324 & Primary & $26,036^{*}$ \\
\hline $\begin{array}{l}\text { Chester and } \\
\text { Horvath (2008) }\end{array}$ & 2005 & 1,452 & $\begin{array}{l}\text { Primary and } \\
\text { Secondary }\end{array}$ & $27,778^{*}$ \\
\hline Li et al. (2012) & 2006 & 1,192 & Primary & $16,622^{*}$ \\
\hline
\end{tabular}

*LCA does not include any recycled material.

software, and Chester and Horvath (2008) used SimaPro software. As can be seen, there is a marked amount of divergence in the results. The reasons for this stem from four central factors: 1) differences in the total weight of vehicles; 2) differences in the weight of individual materials of manufacture; 3) differences in system boundaries (e.g., the inclusion of transportation energy); and 4) whether or not recycled materials were used. As can be seen, the majority of the studies do not use recycled material (as indicated by an asterisk) and, hence, their energy totals are higher. As well, there are discrepancies in the materials which are recycled. For example, Schweimer and Levin (2000) only consider recycled aluminum in their study. 
In addition, in terms of the overall life cycle of a vehicle, variance appears to significantly affect the materials production phase of the life cycle. It is generally accepted that the use phase of a vehicle's LCA shows the largest consumption of energy at over 200MWh per vehicle (McLean and Lave, 1998; Schweimer and Levin, 2000; Chester and Horvath, 2008). However, using the time-series data and modeling in GREET1.8 (Wang, 2012)—which models the fuel cycle phase of a vehicle's life cycle — shows a coefficient of variance of only 5.48\% for the period of 1990 to 2010. This is due to GREET1.8 modeling crude oil refining efficiency as steady across this same period at $98 \%$. Also steady over this period are the lower heating value of gasoline at $32 \mathrm{MJ} / \mathrm{L}$ and a fixed $260,000 \mathrm{~km}$ total distance travelled during the life cycle. Hence, the only factor which contributes to energy variance in this period is the change in vehicle fuel economy which ranged between 9.23 to $10.55 \mathrm{~km} / \mathrm{L}$. Additionally, Sullivan et al. (2012) found that the vehicle assembly phase of the vehicle life cycle inventory shows a coefficient of variance of $8.4 \%$ for energy intensity. Lastly, the end-of-life portion of the vehicle's life cycle usually has the least total energy consumption. Keolian et al. (1997) give energy estimates of $801 \mathrm{~kJ} / \mathrm{kg}$ for the endof-life portion, while Staudinger and Keoleian (2001) estimate $1005 \mathrm{~kJ} / \mathrm{kg}$. Thus, for a $1,500 \mathrm{~kg}$ vehicle, energy total yields are $334 \mathrm{kWh}$ and $419 \mathrm{kWh}$, respectively. As such, these energy are minor in lieu of the overall energy burden across the vehicle's life cycle. Furthermore, most of the end-of-life cycle technology for vehicles (dismantling, shredding, material separation, etc.) has remained largely static (Sakai et al., 2014). Consequently, the most variance in the life cycle of the vehicle is seen in the materials production phase.

With the regular sedan model the mean total energy $(\bar{x})$ is $10,908 \mathrm{kWh}$ and the standard deviation $(\sigma)$ is $6,225 \mathrm{kWh}$ over the full time frame. This yields a coefficient of variation $(C V)$ of $57.1 \%$, which is large. The $C V$ indicates that there is a considerable amount of variation in this distribution. Of course, the variance reflects the probability distribution which was constructed from all the historical data. The corresponding values for the sedan on the five year time frame sees $\bar{x}=9,578 \mathrm{kWh}, \sigma=4,539 \mathrm{kWh}$, and $C V=47.4 \%$. Hence, there is a $12.2 \%$ reduction in total energy usage relative to the full time frame mean $(10,908 \mathrm{kWh})$. However, it should be noted here that some of the individual materials of manufacture saw their total energy rise from the full time frame to the five year time frame (discussed below). Furthermore, there is also a 17.0\% reduction in the coefficient of variation relative to the full time frame value $(57.1 \%)$. For the 
SUV and PUT, there are $12.5 \%$ and $12.7 \%$ reductions in mean total energy (respectively), as well as $16.6 \%$ and $16.1 \%$ reductions in coefficients of variation. The F-test conducted on the full

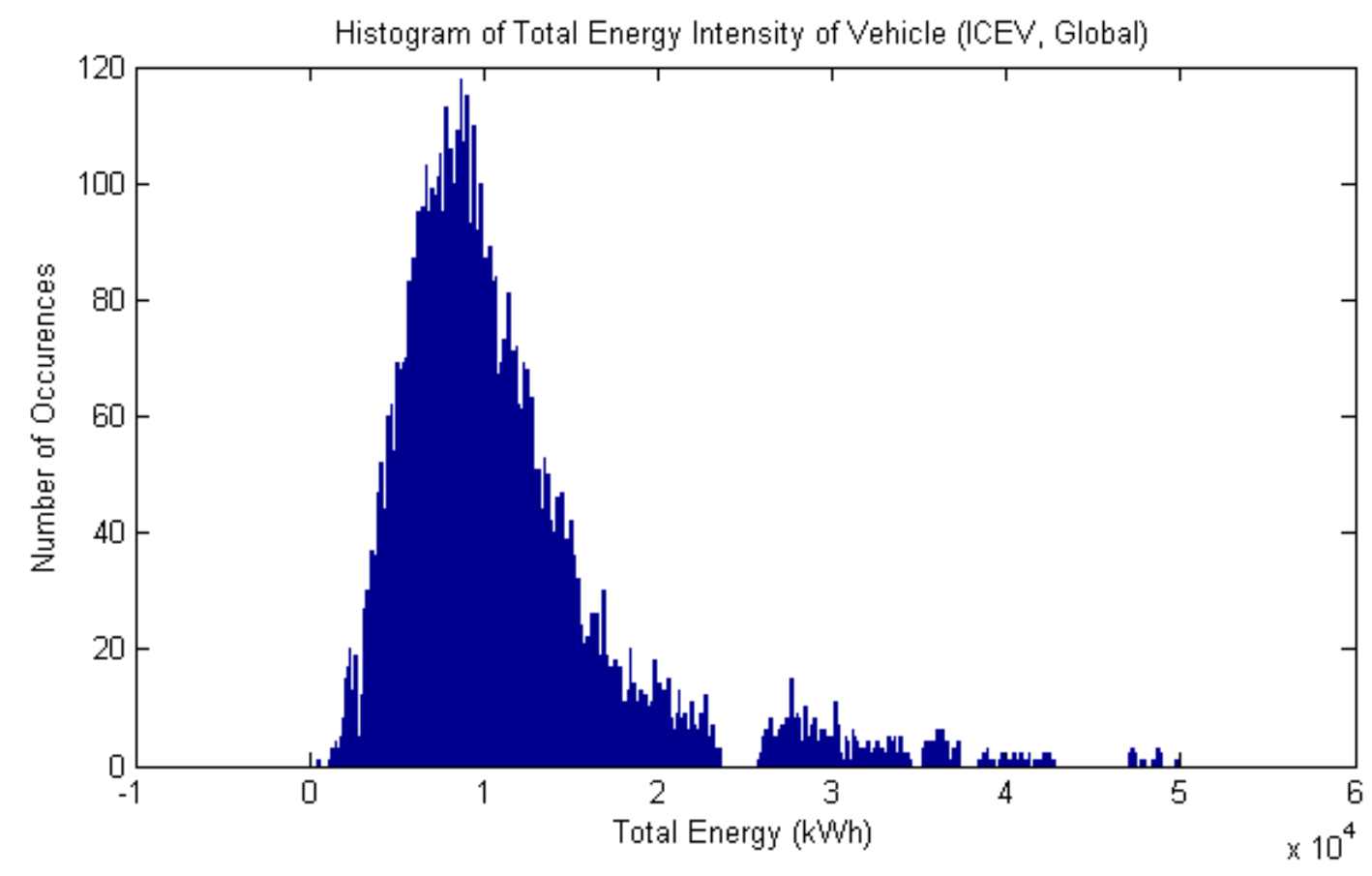

Figure 3.4 An example of a single Monte Carlo distribution for the total energy of an internal combustion engine sedan.

time scale and the five year time scale (2007-2012) for the regular sedan found an F-ratio of 297.6 and, at an $\alpha$ of 0.01, an $F_{\text {crit }}$ of 6.64 (from Excel). As the F-ratio is significantly larger than the $F_{c r i t}$, this indicates that the two distributions are very different.

However, when comparing the two time frames, the lightweight vehicles saw much lesser reductions in mean total energy. The sedan model saw a reduction of 3.85\% in mean total energy and a $13.3 \%$ reduction in the coefficient of variation. The lightweight SUV and PUT models saw reductions of $2.20 \%$ and $2.07 \%$ for the mean total energy, as well as $5.52 \%$ and $5.22 \%$ reductions in their coefficient of variation. An F-test conducted on the full time scale and the five year time scale (2007-2012) for the lightweight sedan found an F-ratio of 35.3 and, at an $\alpha$ of 0.01, an $F_{\text {crit }}$ of 6.64 (from Excel). As the F-ratio is larger than the $F_{c r i t}$, this indicates that the two distributions are different. However, it will be noted that the difference is much less than 
Table 3.7 A breakdown of the total energy $(E)$ of a regular vehicle (sedan) in $\mathrm{kWh}$ and in terms of individual materials of manufacture $(i)$.

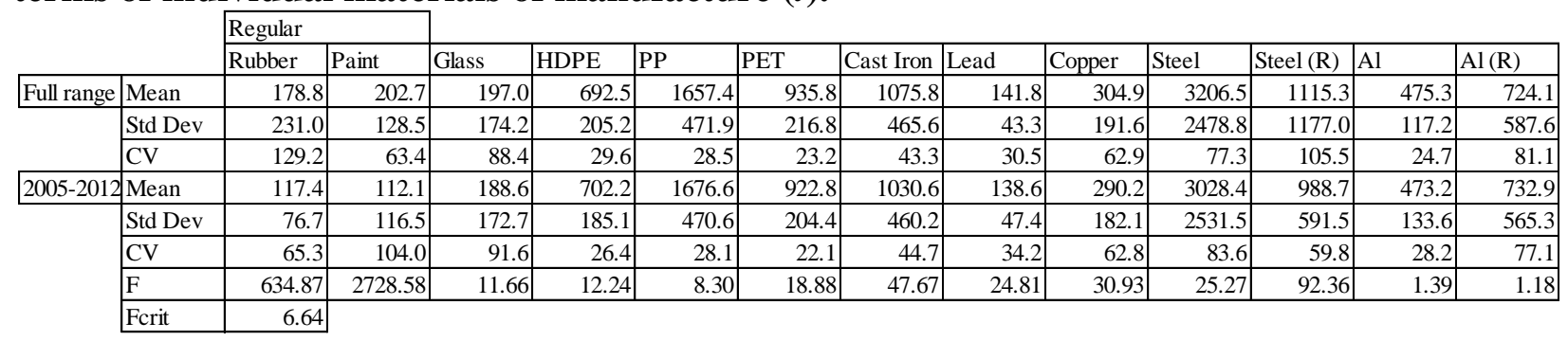

Table 3.8 A breakdown of the total energy $(E)$ of a lightweight vehicle (sedan) in $\mathrm{kWh}$ and in terms of individual materials of manufacture $(i)$.

\begin{tabular}{|c|c|c|c|c|c|c|c|c|c|c|c|c|c|c|}
\hline & \multicolumn{2}{|l|}{ Lightweight } & \multirow[b]{2}{*}{ Glass } & \multirow[b]{2}{*}{ HDPE } & \multirow[b]{2}{*}{ PP } & \multirow[b]{2}{*}{ PET } & \multirow{2}{*}{ Cast Iron } & \multirow[b]{2}{*}{ Lead } & \multirow[b]{2}{*}{ Copper } & \multirow[b]{2}{*}{ Steel } & \multirow[b]{2}{*}{\begin{tabular}{|l|} 
Steel $(\mathrm{R})$ \\
\end{tabular}} & \multirow[b]{2}{*}{$\mathrm{Al}$} & \multirow[b]{2}{*}{$\mathrm{Al}(\mathrm{R})$} \\
\hline & & Rubber & Paint & & & & & & & & & & & \\
\hline \multirow[t]{3}{*}{ Full range } & Mean & 178.8 & 101.3 & 120.3 & 508.0 & 1215.9 & 686.5 & 209.6 & 63.6 & 293.5 & 962.8 & 334.9 & 1039.7 & 1353.2 \\
\hline & Std Dev & 231.0 & 64.2 & 106.3 & 150.5 & 346.2 & 159.0 & 90.7 & 19.4 & 184.5 & 744.3 & 353.4 & 256.4 & 1098.1 \\
\hline & $\mathrm{CV}$ & 129.2 & 63.4 & 88.4 & 29.6 & 28.5 & 23.2 & 43.3 & 30.5 & 62.9 & 77.3 & 105.5 & 24.7 & 81.1 \\
\hline \multirow[t]{5}{*}{ 2005-2012 } & Mean & 117.4 & 33.6 & 102.1 & 508.0 & 1321.8 & 683.6 & 204.1 & 60.6 & 266.8 & 650.4 & 268.7 & 1065.8 & 1481.8 \\
\hline & Std Dev & 76.7 & 34.8 & 17.7 & 74.2 & 338.9 & 75.1 & 97.5 & 28.7 & 180.0 & 603.9 & 134.2 & 416.7 & 1056.4 \\
\hline & $\mathrm{CV}$ & 65.3 & 103.8 & 17.3 & 14.6 & 25.6 & 11.0 & 47.7 & 47.3 & 67.5 & 92.8 & 50.0 & 39.1 & 71.3 \\
\hline & $\mathrm{F}$ & 634.9 & 8593.6 & 283.0 & 0.0 & 477.8 & 2.6 & 17.2 & 70.9 & 107.6 & 1062.5 & 307.0 & 28.5 & 71.2 \\
\hline & Fcrit & 6.64 & & & & & & & & & & & & \\
\hline
\end{tabular}

Table 3.9 A breakdown of the total energy $(E)$ of a regular vehicle (sedan) in $\mathrm{kWh}$ and in terms of individual materials of manufacture $(i)$ by geographical region.

\begin{tabular}{|c|c|c|c|c|c|c|c|c|c|c|c|}
\hline \multicolumn{2}{|c|}{ North America } & Rubber & Paint & Glass & HDPE & $\mathrm{PP}$ & PET & Lead & Copper & Steel & $\mathrm{Al}$ \\
\hline & Mean & 170.2 & 144.2 & 192.7 & 703.8 & 1648.8 & 948.6 & 138.1 & 276.7 & 3117.0 & 478.9 \\
\hline & \begin{tabular}{|l|} 
Std Dev \\
\end{tabular} & 208.9 & 119.0 & 226.9 & 196.6 & 485.4 & 207.3 & 34.9 & 173.2 & 2539.1 & 117.8 \\
\hline & $\mathrm{CV}$ & 122.7 & 82.5 & 117.8 & 27.9 & 29.4 & 21.9 & 25.3 & 62.6 & 81.5 & 24.6 \\
\hline \multicolumn{2}{|l|}{ Europe } & Rubber & Paint & Glass & HDPE & $\mathrm{PP}$ & PET & Lead & Copper & Steel & $\mathrm{Al}$ \\
\hline & Mean & 141.2 & 94.0 & 186.6 & 682.5 & 1690.3 & 942.4 & 141.7 & 281.6 & 2772.1 & 476.4 \\
\hline & \begin{tabular}{|l|} 
Std Dev \\
\end{tabular} & 172.5 & 96.0 & 176.0 & 191.8 & 391.8 & 176.7 & 51.7 & 191.0 & 2326.7 & 131.1 \\
\hline & $\mathrm{CV}$ & 122.1 & 102.1 & 94.3 & 28.1 & 23.2 & 18.8 & 36.5 & 67.8 & 83.9 & 27.5 \\
\hline \multicolumn{2}{|l|}{ Oceania } & Rubber & Paint & Glass & HDPE & PP & PET & Lead & Copper & Steel & $\mathrm{Al}$ \\
\hline & Mean & 150.8 & 148.8 & 177.2 & 696.7 & 1693.9 & 938.5 & 136.1 & 278.8 & 2819.9 & 477.1 \\
\hline & Std Dev & 195.3 & 118.3 & 174.5 & 195.5 & 396.8 & 185.8 & 49.3 & 185.8 & 2492.3 & 135.7 \\
\hline & $\mathrm{CV}$ & 129.5 & 79.5 & 98.5 & 28.1 & 23.4 & 19.8 & 36.2 & 66.6 & 88.4 & 28.5 \\
\hline & $\mathrm{F}$ & 58.6 & 742.3 & 16.2 & 30.9 & 34.5 & 7.2 & 37.6 & 1.84 & 58.0 & 1.01 \\
\hline & Fcrit & 4.61 & & & & & & & & & \\
\hline
\end{tabular}

Table 3.10 Log transformed version of Table 3.9.

\begin{tabular}{|c|c|c|c|c|c|c|c|c|c|c|c|}
\hline \multicolumn{2}{|c|}{ North America } & Rubber & Paint & Glass & HDPE & $\mathrm{PP}$ & PET & Lead & Copper & Steel & $\mathrm{Al}$ \\
\hline & Mean & 2.69 & 2.17 & 2.09 & 2.87 & 3.21 & 2.99 & 2.12 & 2.36 & 3.45 & 2.70 \\
\hline & Std Dev & 0.22 & 0.43 & 0.46 & 0.13 & 0.16 & 0.12 & 0.02 & 0.27 & 0.18 & 0.05 \\
\hline \multirow[t]{3}{*}{ Europe } & & Rubber & Paint & Glass & HDPE & $\mathrm{PP}$ & PET & Lead & Copper & Steel & $\mathrm{Al}$ \\
\hline & Mean & 2.04 & 1.93 & 2.23 & 2.83 & 3.22 & 2.97 & 2.13 & 2.32 & 3.39 & 2.68 \\
\hline & Std Dev & 0.31 & 0.48 & 0.29 & 0.16 & 0.10 & 0.09 & 0.22 & 0.42 & 0.28 & 0.10 \\
\hline \multirow[t]{5}{*}{ Oceania } & & Rubber & Paint & Glass & HDPE & PP & PET & Lead & Copper & Steel & $\mathrm{Al}$ \\
\hline & Mean & 2.06 & 2.17 & 2.20 & 2.86 & 3.22 & 2.97 & 2.09 & 2.31 & 3.39 & 2.68 \\
\hline & Std Dev & 0.35 & 0.43 & 0.27 & 0.13 & 0.12 & 0.10 & 0.20 & 0.41 & 0.28 & 0.10 \\
\hline & $\mathrm{F}$ & 15400.2 & 904.2 & 423.7 & 233.3 & 37.8 & 135.1 & 137.2 & 49.8 & 215.9 & 113.7 \\
\hline & Fcrit & 4.61 & & & & & & & & & \\
\hline
\end{tabular}




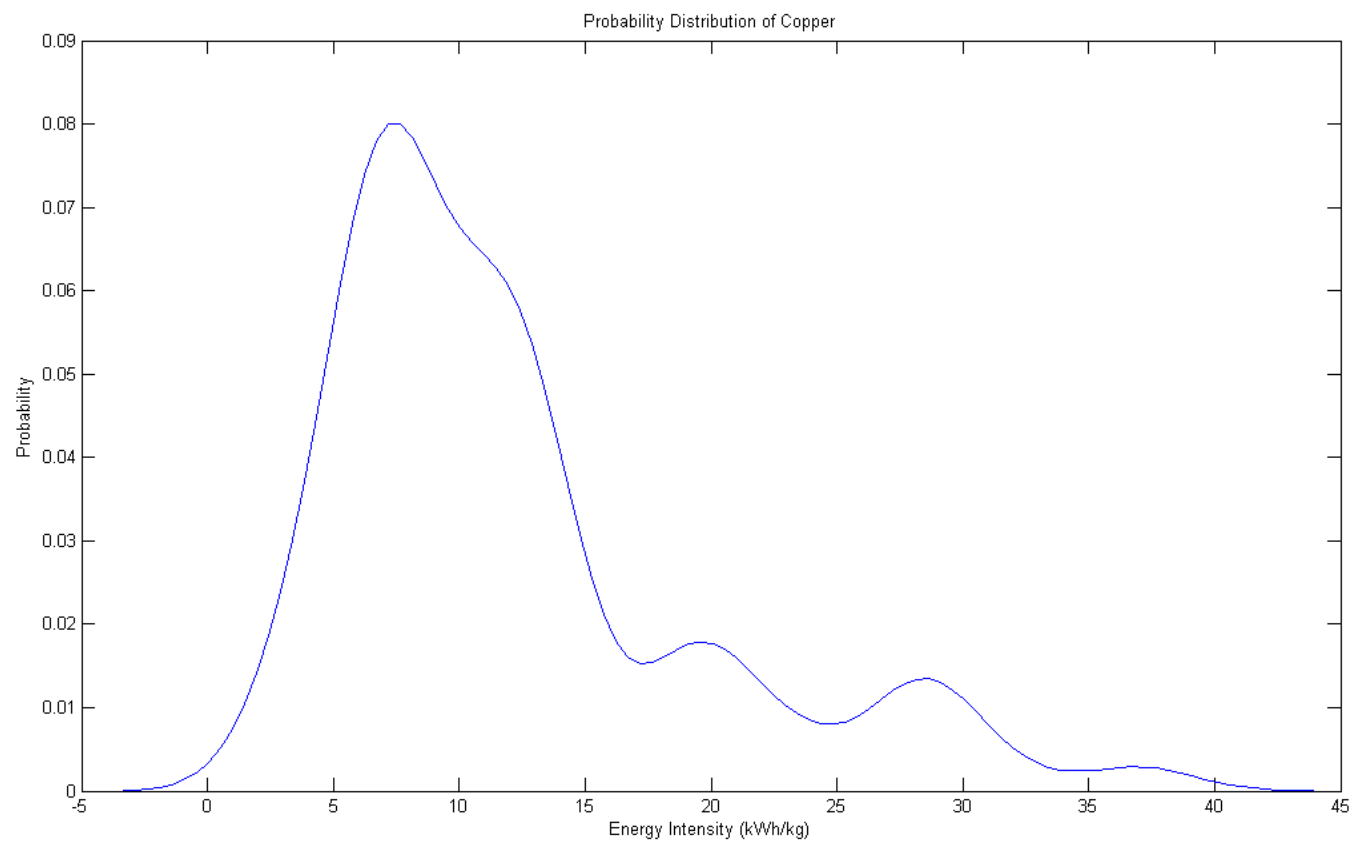

Figure 3.5 A sample probability distribution for the energy intensity of copper.

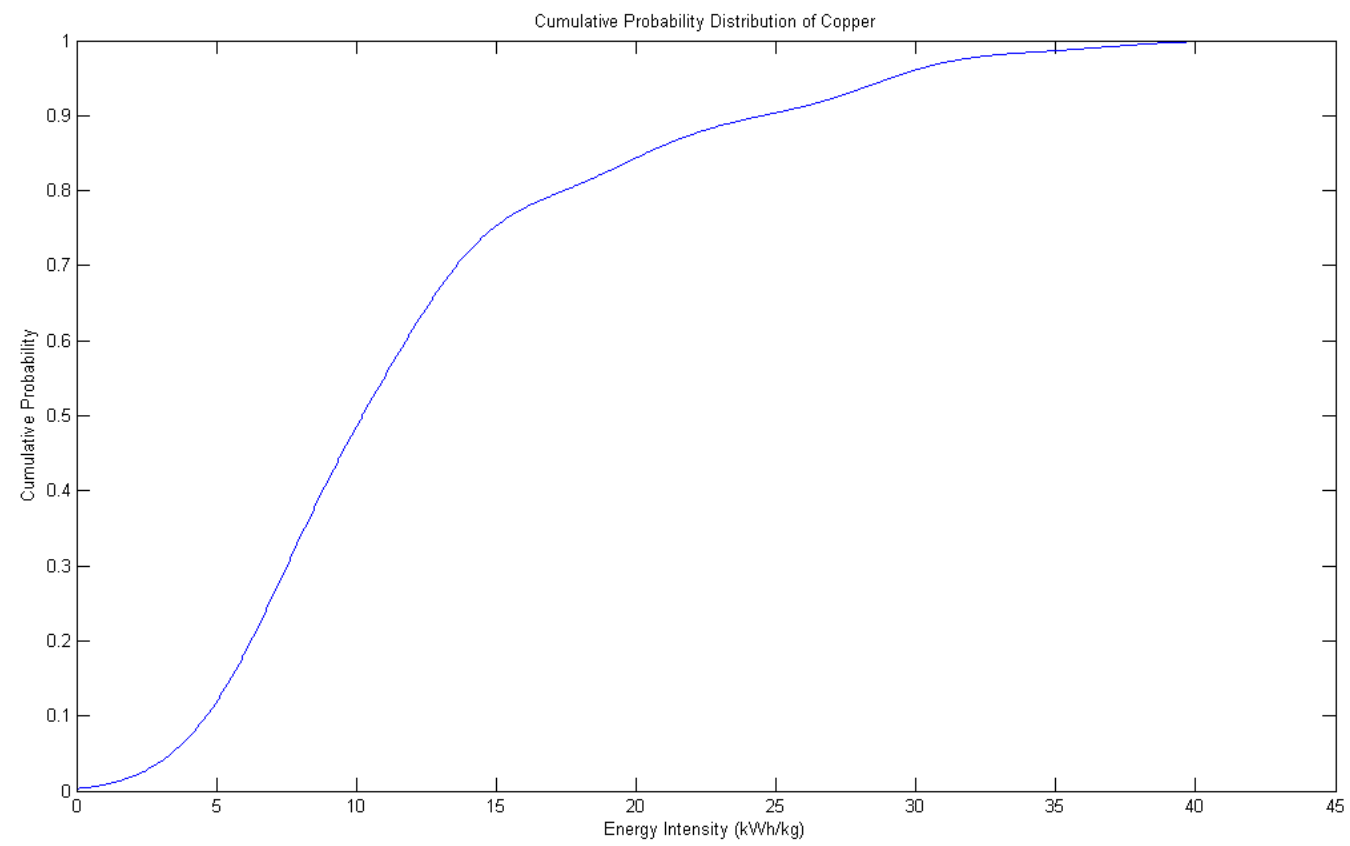

Figure 3.6 A sample cumulative probability distribution for the energy intensity of copper. 


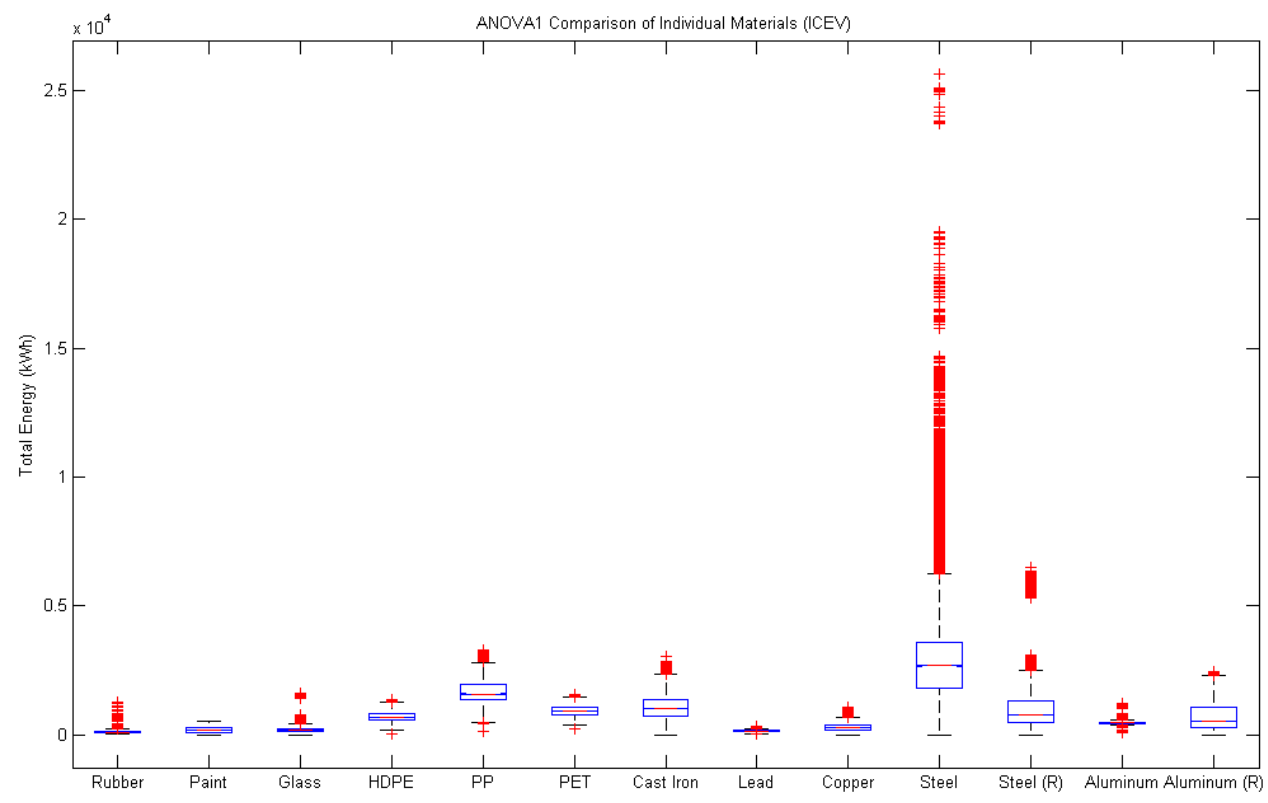

Figure 3.7 A one-way ANOVA of an internal combustion engine sedan using regular materials.

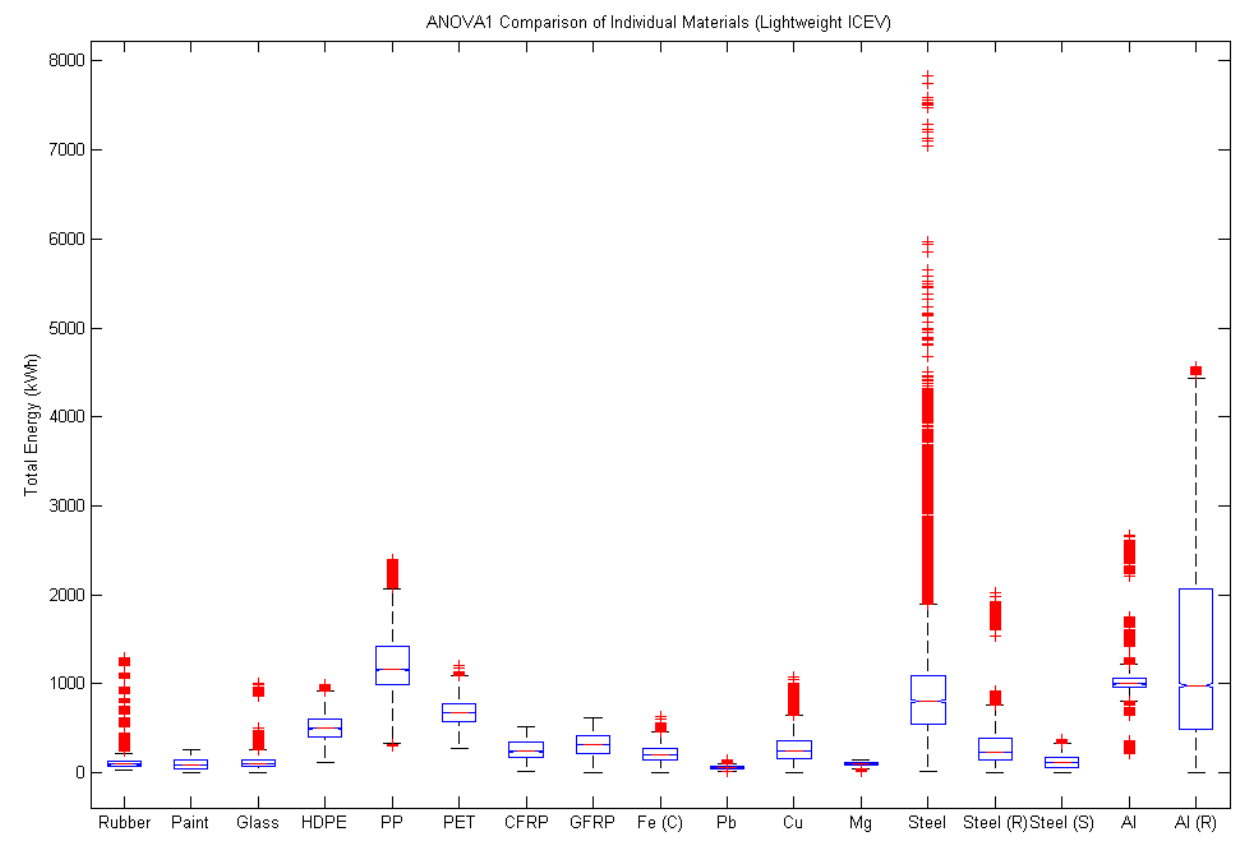

Figure 3.8 A one-way ANOVA of an internal combustion engine sedan using lightweight materials. 
the regular sedan vehicle. Part of this is owing to the negligible temporal differences in energy contributions from magnesium, stainless steel, CFRP, and GFRP. Yet, for the remaining materials, the shifts in total mean energy and coefficients of variation have a lot to do with the types of materials selected and their corresponding weight. In terms of weight, the regular vehicles use three times as much steel, recycled steel and stainless steel than the lightweight vehicles. Regular vehicles also use more than four times as much cast iron. Conversely, lightweight vehicles use more aluminum, recycled aluminum, and plastics than the regular vehicles. This subject is further elaborated below.

Utilizing box plots derived from a one-sided ANOVA indicates that for a regular sedan in the full time frame, the key contributor to this variance in regular vehicles comes from virgin steel with polypropylene (PP), recycled steel, cast iron, and recycled aluminum, also adding a fair amount to the size of the distribution (Figure 3.7). Furthermore, the ANOVA also reveals that the skewness of the Monte Carlo distribution in Figure 3.4 (the shift in the plot towards the vertical axis, or left) is due mainly to the large variance and high number of outliers seen in the steel box plot. Steel is also a serious contributor to the variance of the total energy seen in heavier vehicles such as sport utility vehicles (SUVs) and pick-up trucks (PUT). In the case of lightweight vehicles (Figure 3.8), the box plots reveal that the largest contributor to the variance in the full time frame was due to the amount of recycled aluminum. Virgin steel and polypropylene were the second highest contributors to total energy. The main reason for this reduction in energy is due to the high proportion of recycled aluminum in the vehicle's body (13.1\% versus $4.9 \%$ for the regular materials version). This recycled aluminum by-passes primary production through the highly energy-intensive Hall-Héroult process of converting alumina (A12O3) into elemental aluminum.

\subsubsection{Discussion related to the use of intervals for Monte Carlo simulations}

Nevertheless, when examining their individual contributions, materials such as aluminum, recycled aluminum and the plastics (HDPE, PP, PET) show not very significant changes between the two time frames in their mean energy values $(\mathrm{kWh})$ in Tables 3.7 and 3.8. Here, it can be seen that paint and rubber show the most reductions in mean energy $(\mathrm{kWh})$ for both the regular 


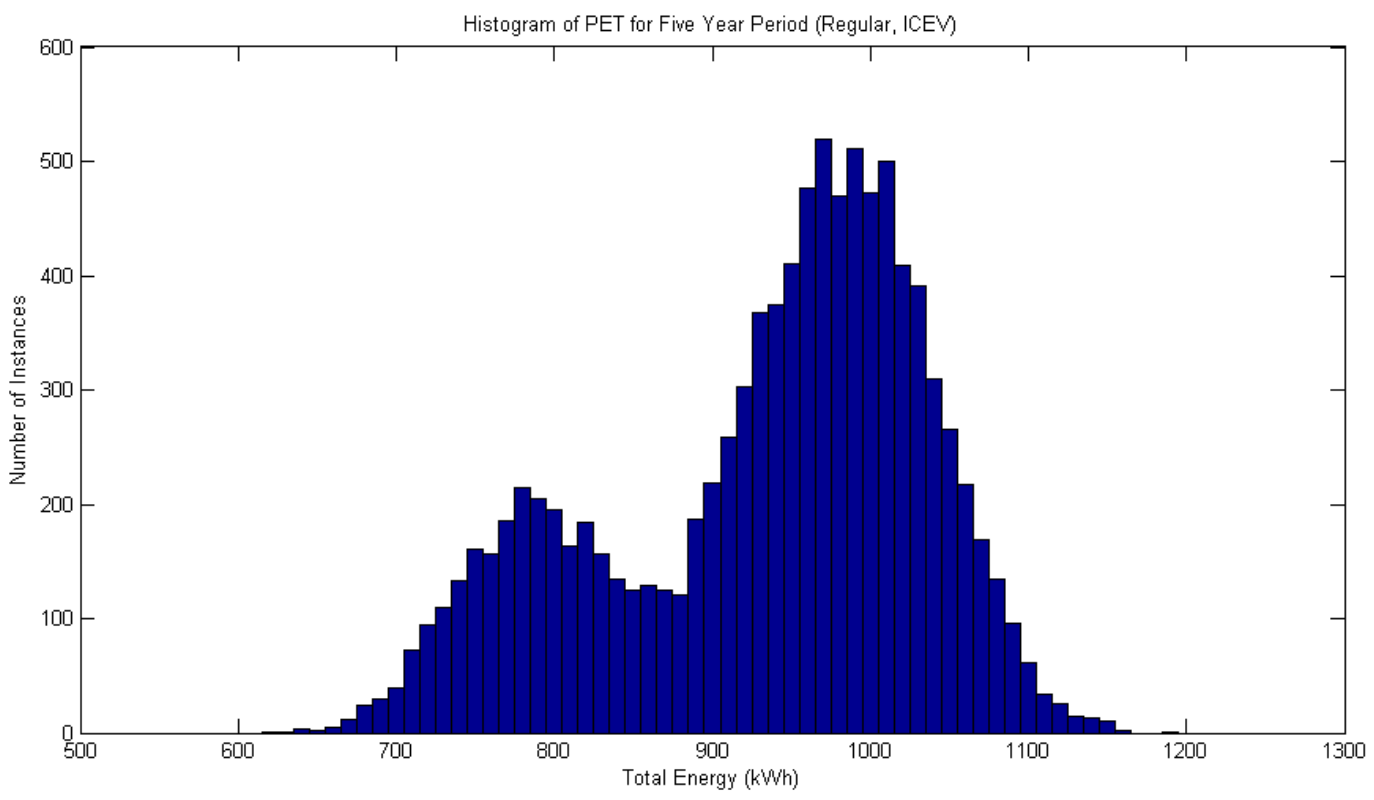

Figure 3.9 Histogram of PET energy of a regular sedan for the Five Year Period (20072012).

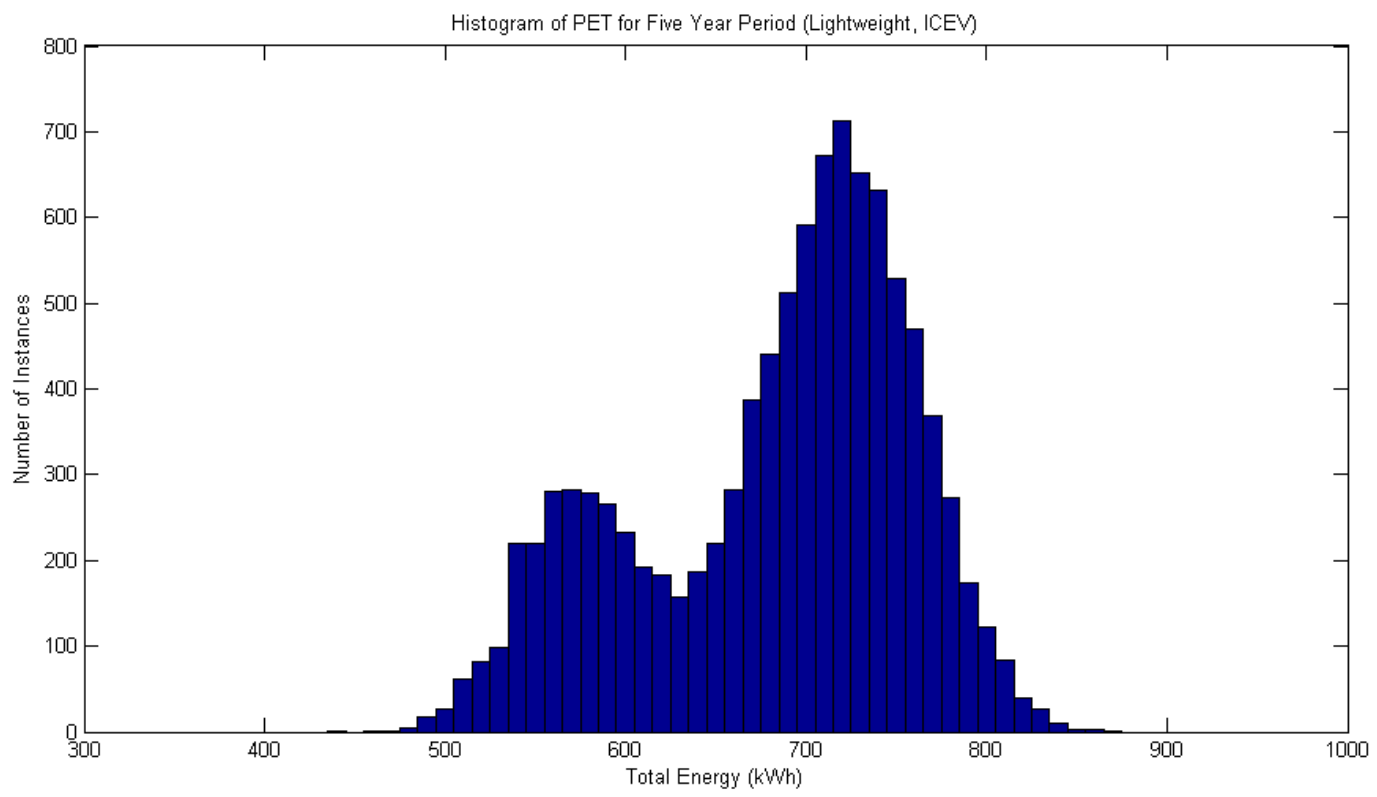

Figure 3.10 Histogram of PET energy of a lightweight sedan for the Five Year Period (2007-2012). 


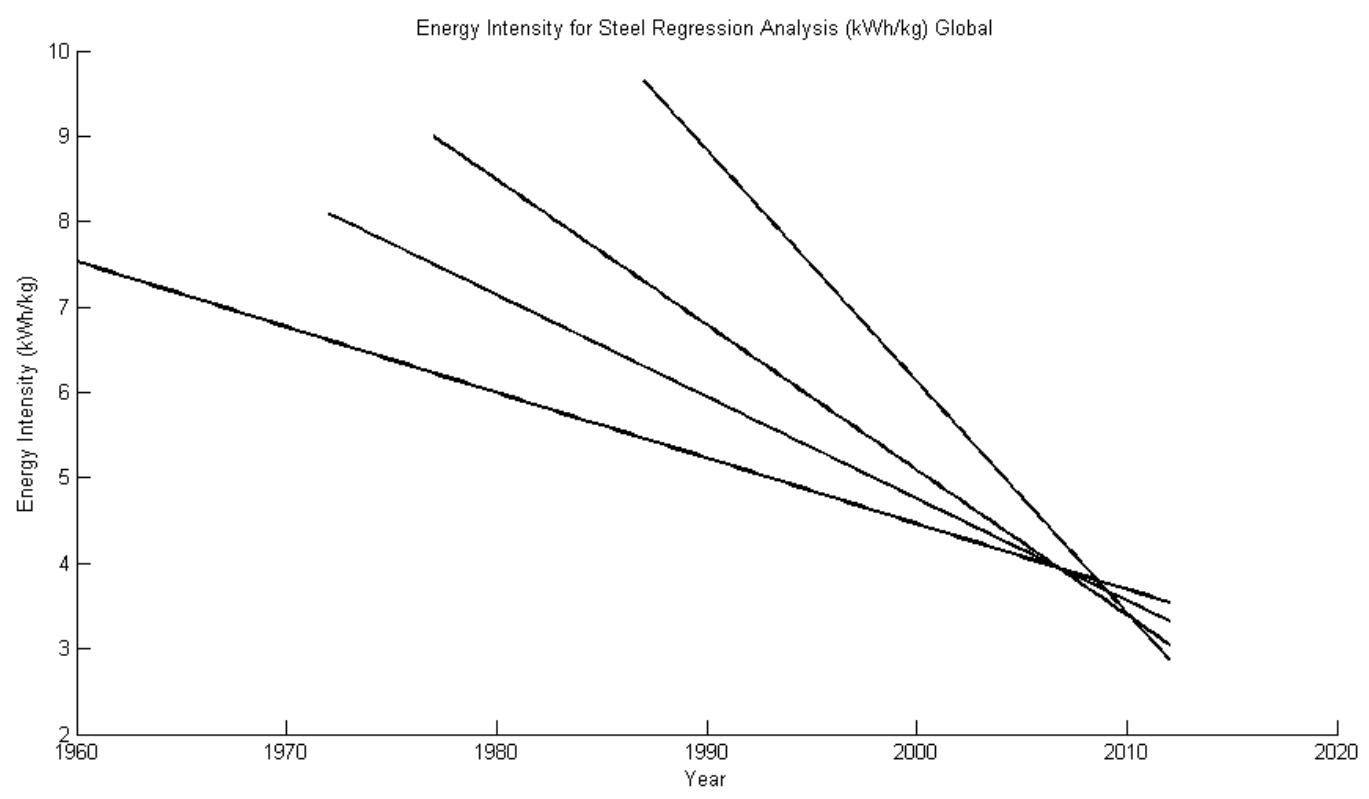

Figure 3.11 Energy intensity $(\mathrm{kWh} / \mathrm{kg})$ for steel over various time intervals.

and lightweight vehicles. (Note that for rubber, the mean energy values remain the same for both types of vehicles as they use the same weight.) It will be seen that the standard deviations and coefficient of variation for the materials of manufacture are initially all the same in the full time scales, regardless of which vehicle type is considered. However, this is not the case when examining the five year time frames for both regular and lightweight vehicles. For example, PET has an initial CV of $23.2 \%$ for both vehicle types but, in the five year data range, has $22.1 \%$ and $11.0 \%$ for regular and lightweight vehicles respectively. This would indicate that the effects of temporality in this instance are not scalable. (The only exception found here is with rubber, which has the same weight for both lightweight and regular vehicles.) That is, scalar reductions in mean energy values do not see corresponding scalar reductions in standard deviations. This is problematic because the whole basis for using coefficients of variation is that changes in mean values correspond to scalable changes in standard deviations (Sørensen, 2002). For example, this scalar quality was seen with the full time frame because the $C V$ values were exactly the same in the regular and lightweight models. Nevertheless, histograms of the energy contributions for each of the individual materials in the five year distributions for each of the regular and lightweight vehicles are identical and, thus, reproduce their probability distribution. For instance, the histograms of the five year PET distributions are identical even though their $C V$ 
values are different. Because the $C V$ has the effect of eliminating variation in mean energy values, it would be expected that these final $C V$ values were the same if the standard deviations were scalar to the mean. Obviously they are not. Also, there is no a priori evidence to suggest that this is expected. Hence, analysis utilizing $C V$ values between time intervals-especially small time intervals - is not a recommended method of analysis. Thus, to return to the subject of time scale, the particular portions of Tables 3.7 and 3.8 which should be considered are those with the full time scale. Doing so allows for comparison of $C V$ values between different materials because they are scalable. As such, it does not matter whether a regular or lightweight model is selected since the $C V$ values are the same. Thus, Tables 3.7 and 3.8 indicate that rubber has the most amount of variation (129.2\%) and magnesium the least (21.4\%). Naturally, this comes with the caveat that each of these values corresponds to a different time frame in Table 3.2. Nevertheless, this is purposeful since it is an indicator of overall variation due to time and to what degree each material is affected by this variation. The origins of this problem have partly to do with the particular intervals that the data are being filtered through. The fact that the mean energy totals, standard deviations, and coefficients of variation are not significantly reduced (or increased) may have as much to do with the particular characteristics of the data in the time interval itself. For example, Figure 3.11 shows four regression lines for four different time intervals for steel. The horizontal length of the line corresponds to the years the interval covers. Thus, all the regression lines terminate at 2012 but commence at different times: 1960, 1972, 1977, and 1987. These particular intervals were selected to illustrate that there can be more variation in energy intensity $(\mathrm{kWh} / \mathrm{kg})$ even in smaller time intervals as by their vertical lengths. Thus, mean energy values and standard deviations for smaller time intervals can have reduced significance when compared to the full time scale. Consequently, it is recommended that only a full interval analysis be done with the standard deviation of these intervals as the key metric of interpretation. It should be noted here that, as an indicator of variation, the standard deviation is not relative to the arithmetic mean. At the very least, this suggests that a parsimonious regression method should be employed on all individual materials first in order to characterize temporal changes in energy intensity values rather than primarily conducting an analysis from the perspective of the functional unit. Such a method as the least absolute residuals (LAR) should be used in order to make recommendations with regard to updating LCI data, as well as to verify the source of the variance. This will partly be the subject of the next chapter. Naturally, 
whether there is any benefit between LAR and more conventional methods such as least-squares will be examined.

\subsubsection{Discussion on the possibility of non-normality in the temporality results}

Another problem with analyzing these data has to do with the type of distributions resulting from the Monte Carlo simulation. The visibly apparent skewness of the total energy distribution in Figure 3.4 suggests that the correct way to deal with the problem of lack of scalability is to $\log$ transform the data. Indeed, when the data is log transformed, there is a significant reduction in the disparities between $C V$ values in the two time frames (see Tables 3.9 and 3.10). However, this is expected because log transforming the data has the effect of reducing the influence of outliers in the probability distribution. This should have the effect of lowering F values by increasing the similarity in distributions. However, this is not always the case. In some instances, there is an increase in F values after the data has been log transformed. This means that the log transformed distributions are less similar than the non-log transformed distributions. In such a case, this would mean that the original distribution is not log-normal to an appreciable degree (see below discussion on kurtosis and skewness). Nevertheless, there is utility in doing this to test for the assumption of scalability for individual materials. From a practical perspective, this should be done not only to see whether individual material distributions are skewed or not, but also to see whether the effects of these skewed distributions on the F values are significant or not. This should be done by testing whether there is a reduction in the kurtosis and skewness of the distribution after the data has been log transformed. As embedded functions in MATLAB, this is easily facilitated and will be discussed as an example in the spatiality results below.

\subsubsection{Monte Carlo simulations and analysis of variance (spatiality)}

Because of the aforementioned data limitations, spatiality in this preliminary study was limited to three geographical regions: North America, Europe and Oceania. North America comprises Mexico, USA and Canada. Europe consists of the European Union (EU), former Soviet Bloc countries, as well as Norway, Switzerland, Turkey and Serbia. Lastly, Oceania consists of 
Australia and New Zealand. For convenience, the geographic zones covered under the temporality and spatiality studies are given below in Figures 3.12 and 3.13. Countries covered are indicated in black. Again, as with the above calculations, energy data from primary production was considered here only because of the ramifications of the many different types of secondary production. Hence, there were no alterations in the characteristics between data coming from different regions. Regional results from Table 3.5 for a regular ICEV sedan are as follows: North America ( $\bar{x}=11,047 \mathrm{kWh}, \sigma=5,868 \mathrm{kWh})$, Europe $(\bar{x}=10,347 \mathrm{kWh}, \sigma=5,874 \mathrm{kWh})$ and Oceania $(\bar{x}=10,463 \mathrm{kWh}, \sigma=6,081 \mathrm{kWh})$. The coefficients of variance $(53.1 \%, 56.8 \%$, and $58.1 \%$, respectively) indicate that there are effects owing to spatiality since the time frame under consideration is the same. The F-test conducted on these three distributions indicated an F-ratio of 39.87 and, at an $\alpha$ of 0.01 , the $F_{\text {crit }}$ was 4.61 . This indicates that the expected values in the three distributions are different. The box plots derived from an ANOVA1 of the total energy (i.e., at the level of a functional unit) showed little difference from region to region and, for that reason, they are not included here. From Table 3.9, it will be noted that the differences in the total energy values in Table 3.5 are due to the cumulative simultaneous effects of individual materials. It will be seen from the F values at the bottom of Table 3.9 that there is no significant difference in distributions for copper and aluminum. However, at the same time, most of the differences between distributions occur with rubber, paint, and steel. This indicates that the effects owing to spatiality are significant only in the case of some materials and not all. Moreover, this shows that any consideration of temporality due to age of data must be simultaneously framed in spatial terms as well.

Turning to the prescribed log transformation issue, when the spatial distributions in Table 3.9 are $\log$ transformed in Table 3.10, there is an across the board increase in F values. This would seem to suggest that the differences in the distributions are actually more significant. Unfortunately, this is not as clear as this would indicate, as an examination of kurtosis demonstrates. A kurtosis greater than 3.00 indicates that the distribution is more outlier prone than a normal distribution (MathWorks, 2015). Hence, greater kurtosis values indicate greater peaked distributions. An examination of the kurtosis of the individual material distributions in Table 3.9 and 3.10 shows two different phenomena: on the one hand, the kurtosis is increased following log transformation and, on the other hand, the kurtosis is decreased but remains at an 
unacceptable high level. Thus, an example of the former phenomenon sees the kurtosis increase from 3.04 to 5.06 for North American high density polyethylene (HDPE). Conversely, an example of the latter phenomenon sees the kurtosis decrease from 21.2 to 8.40 for European steel. Hence, while this is a reduction in the level of kurtosis for HDPE, this hardly brings about a desirable outcome. The sole exception to either of these phenomena was with European polypropylene where the kurtosis decreased from 2.96 to 2.50 after being log transformed. Similarly, log transforming the spatiality data saw overall reductions in skewness of the distributions. However, this was minimal in terms of its overall effect. For example, the skewness of the North American steel distribution was reduced from 6.41 to 1.12 when $\log$ transformed. Conversely, other materials showed a shift from a positive skew to a negative skew when $\log$ transformed, such as European cast iron which saw a shift from 0.575 to -2.187. Ideally, log transforming data should result in bringing about a skewness closer to zero. Consequently, when analyzing the $\mathrm{F}$ values between different spatial least absolute residuals distributions, there is little added value in log transformed individual materials distributions unless there is a demonstrated correction for skewness. This was not found to any significant level in this study.

\subsection{Conclusions}

This study contributed to the literature examining the potential for temporal and spatial effects in life cycle assessment due to age of data. No previous study has directly examined this issue with the aim of assessing the potential impacts of using source data rooted in a specific time and place. The consequence of this has been that life cycle assessment studies are tacitly accepted without any particular specification as to the duration of their validity. This is not to state that any LCA suddenly becomes invalid, but that the issue of raw data upon which they are based has to be renewed. This study also serves a preliminary normative platform for moving forward in examining the issue of temporality and spatiality in life cycle assessment. It established that Monte Carlo simulations can provide a useful tool for examining the individual variance of materials used within a functional unit, which may be visually rendered in the form of box plots. All 17 materials of manufacture were found to show varying degrees of variation across their 
respective time frames. However, comparing the variance (including $\mathrm{F}$ values) across time intervals did not prove to be particularly useful since small time intervals were found to have just as much variance as larger intervals. Coefficients of variation changed between intervals which is indicative of a non-scalability. This lack of scalability was of particular concern since it is highly suggestive of non-normal data. It should be noted here that scalability was present when the full time frame was under consideration because, although the mass of individual materials of manufacture was different for each vehicle type, their corresponding coefficients of variance remained the same. Hence, a test for non-normality was conducted by log transforming the raw data and re-running the Monte Carlo simulations. Utilizing skewness and kurtosis of the individual materials of manufacture distributions, showed that the log transformed distributions did not resolve any non-normality issues and, consequently, were of little use. Nevertheless, this is recommended as part of the platform for further examinations in this area using Monte Carlo simulations since not all trend relationships are innately linear. Arising from this conclusion is that a further regression analysis be utilized in order to test that the variance measured is actually due to a temporal trend. For the spatiality portion of this study, differences between distributions were examined after Monte Carlo simulations were run for the three separate geographic regions: North America, Oceania and Europe. By using analysis of variation (F tests), it was found that energy distributions of most materials do differ significantly by geographic region while copper and aluminum do not show any significant variation by region. 


\section{World Map}

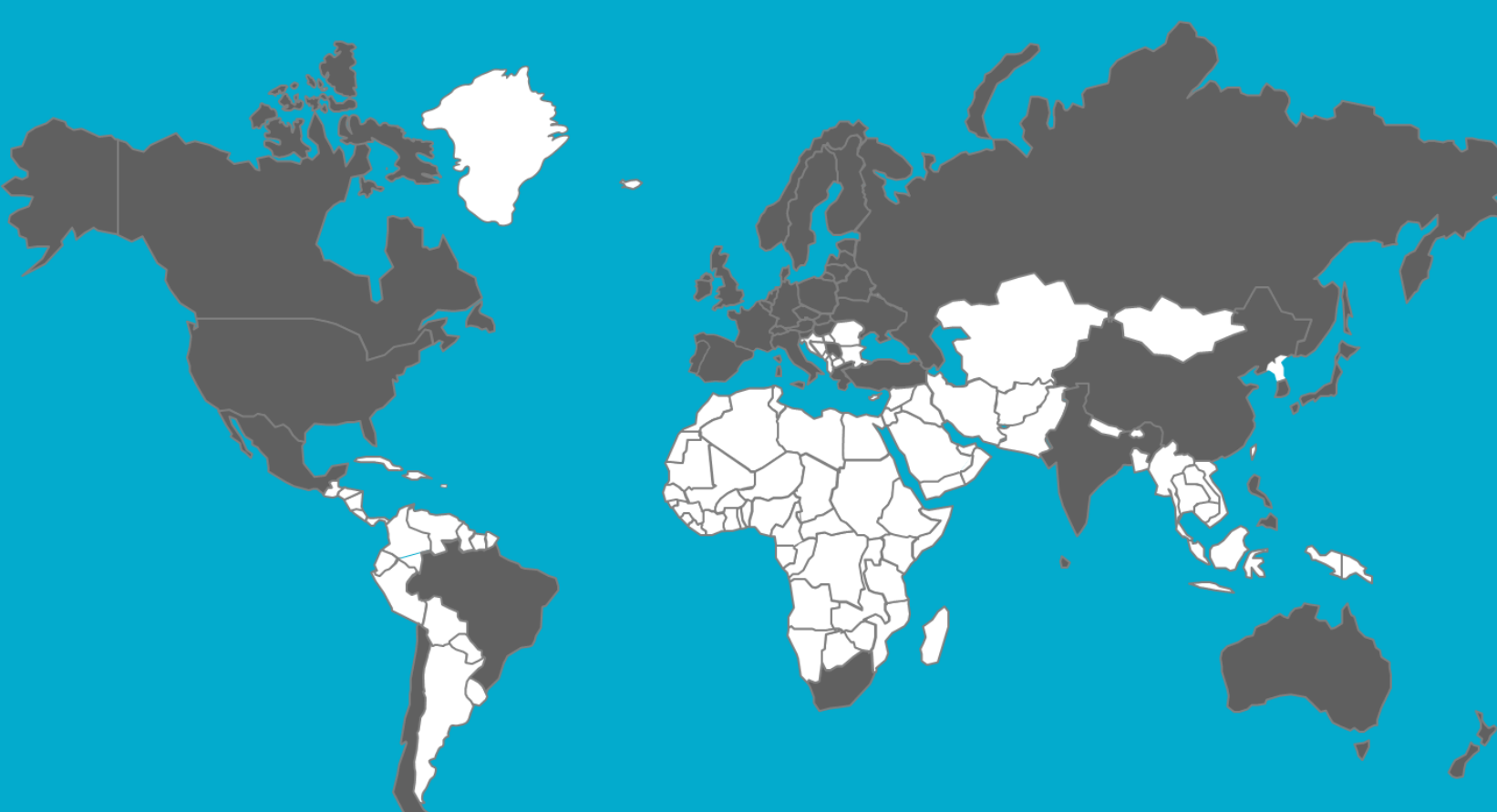

Figure 3.12 Countries studied for temporality due to age of data (see Appendix $\mathrm{C}$ for complete list). 


\section{World Map}

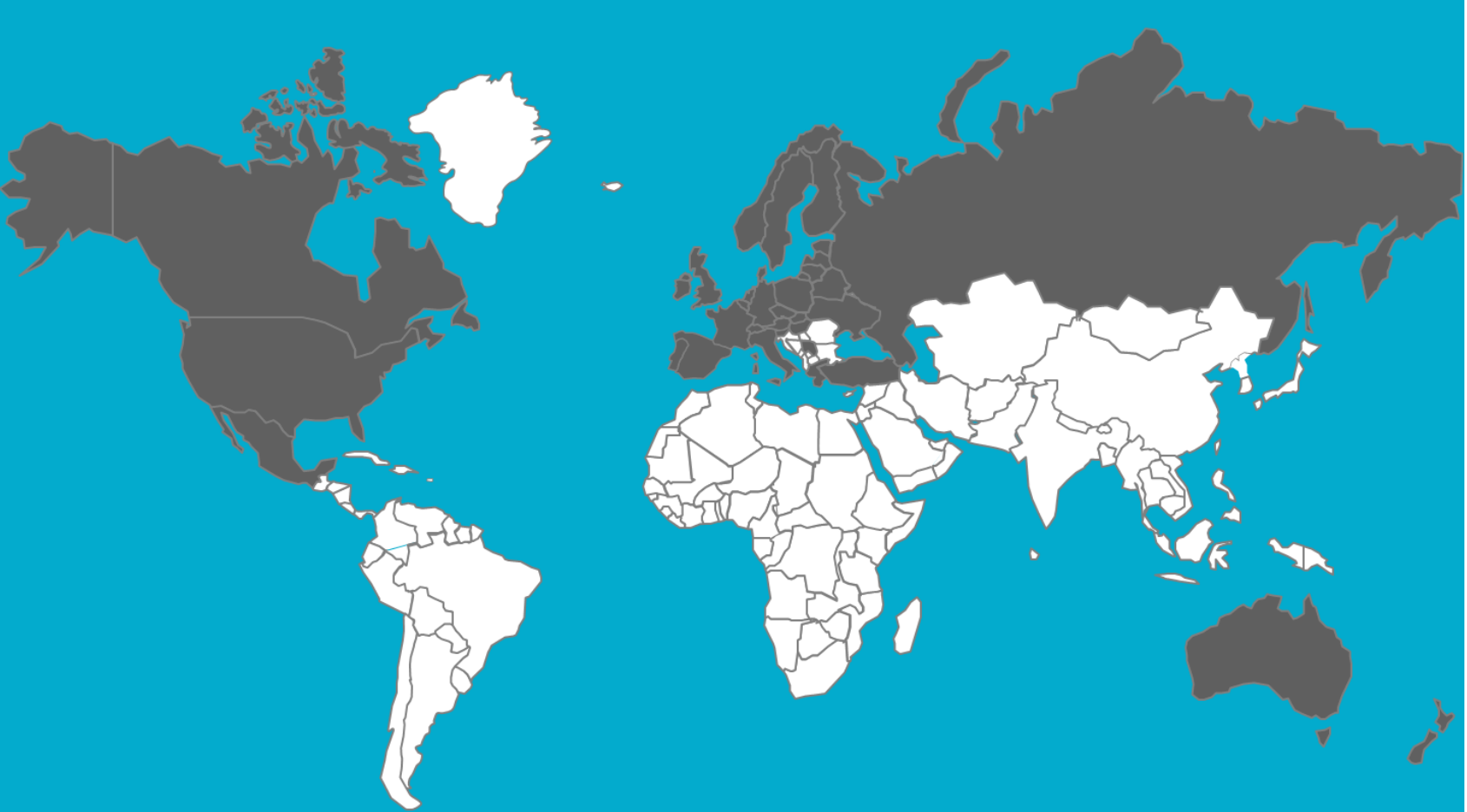

Figure 3.13 Countries involved in the spatiality analysis (see Appendix $\mathrm{C}$ for complete list). 


\section{CHAPTER 4-MONTE CARLO SIMULATIONS AND REGRESSION ANALYSIS FOR ASSESSING TEMPORALITY DUE TO AGE OF DATA}

\subsection{Introduction}

Because life cycle assessments (LCA) are modelled under steady state conditions (Udo de Haes, 2006), temporality has been lacking in traditional life cycle assessments. In this study, temporality relates to the age of data. Thus, temporality in this study does not relate to unit processes and their linked intermediate flows, as well as the integration of emissions to a single point in time. That said, steady state systems operate under the assumption that its internal properties are non-transient and, thus, do not alter internal working conditions. The study of temporality due to age of data has specific implications for the validity of LCA studies by the introduction of both variance and uncertainty. As stated in the previous chapter, variance stems from the innate heterogeneity evidenced within a population or in data. Thus, a homogeneous population or group of data would imply that there is uniformity and, consequently, no variation. Conversely, uncertainty can be understood to stem from missing data, as well as errors in data such that the data could be thought to be non-representative (Ibid.). Therefore, temporality due to age of data would mean that there is variance, and not uncertainty, occurring. The lack of variance found in the regression analysis will be due to a lack of variance rather than uncertainty, since it is assumed that each data point is valid (in situ). Or, the case may be, uncertainty is occurring, but this is due solely to missing or inadequate amounts of data. In fact, this will be seen below in some circumstances for individual materials of manufacture.

As noted in the preceding chapter, temporality is a large subject area with numerous implications for LCA results and processes. Held and Klöpper (2000) noted that temporality in LCA could include the age of data utilized, the lifespan of a product (from cradle to grave), the use and renewal of resources, and so forth. Phungrassami (2008) also notes that this could extend further into the goal and scope definitions used in LCA. The specific focus of this study is on temporality due to the age of data, which is a very specific facet of temporality within LCA 
studies. The lack of inclusion of temporality and spatiality in LCA studies has been previously documented (Reap et al., 2003, 2008a, 2008b). Existing studies of temporality in LCA focus primarily on emissions contributing to greenhouse gases or global warming potential (Levasseur et al., 2010; Säynäjoki et al., 2012; Levasseur et al., 2012). More recently, temporality has figured in LCAs in what is termed dynamic LCAs (e.g., Hendrickson et al., 2006; Stasinopoulos et al., 2012; Beloin-Saint-Pierre et al., 2014). However, dynamic LCAs consider the implications of material flows across time. Thus, this is not reflected in this study as temporality due to the age of data consists of discreet data points. Stated another way, temporality due to age of data is the "temporal dependency of data" (Fleisher et al., 2004). As such, this study focuses on the effects of this type of temporality during the input phases (cradle to gate) of the production of automobiles as modelled by GREET2. Developed by the Argonne National Laboratory, Greenhouse gases, Regulatory Emissions, and Energy use in Transportation (GREET2) models the LCA of automobiles, sport-utility vans (SUV) and pick-up trucks (PUT) through their entire life cycle, from cradle to grave. GREET2 also models different power train configurations, as further discussed below.

The choice of the GREET2 LCA model was predicated on a number of conveniences it provided. Among other things, this included the fact that GREET2 was free, in Microsoft Excel format, and had a considerable body of supporting literature. However, more significantly, because of the number of different power train and material configurations, comparative analyses could be more easily facilitated. Also important was that GREET2 had sufficient complexity. For the purposes of this study, complexity is related to the plurality of individual variables used in modelling (Weaver, 1948). Hence, GREET2 may be considered complex by the substantial number of variables concerned. Also relevant to this study is the notion that the amount of complexity in an object or system is inversely related to the probability that it could have arisen by chance alone (Dawkins, 1986). Thus, an automobile as modelled by GREET2 is more complex as it has a much lower probability of having occurred randomly than, for example, wood chips or quarried gravel. Both concepts of complexity described by Weaver (1948) and Dawkins (1986) are pertinent to this study in order to reduce the risk of anomalous and circumstantial findings. 
The purpose of this study is to determine the effect of temporal variability in the energy intensity of raw materials which go into the manufacturing of automobiles as modelled by GREET2. Energy intensity, or energy use, is a common impact category in LCAs to measure performance and environmental impact (Pelletier et al., 2007). Energy intensity encompasses the production of raw materials from cradle to gate. As such, it is the measurement of the quantity of energy required per unit of output or activity (US Department of Energy, 2012b). This may be compared with embodied energy, which is the amount of energy across the entire life cycle of a product, including manufacturing, transportation and disposal (US Department of Energy, 2012a). In some instances, embodied energy has also been construed to include energy recovery. These two terms, energy intensity and embodied energy, are sometimes used interchangeably, although that is technically not accurate. This current study builds on the previous chapter which focused on internal combustion engine power trains and the manufacturing materials in the upper half of Table 4.1 (which also contains all the acronyms for the raw materials in this study). However, in 2012 GREET2 added a number of additional materials to their LCA platform which are documented in the lower half of Table 4.1. Thus, this study more than doubles the number of materials studied from 17 to 35 . It was shown in the earlier chapter that the effects of temporality were large, given the breadth of time studied (roughly 1970 to 2012). Many times data sources used in LCA studies are considered valid even though they may be dated.

This study builds on methods developed in the earlier chapter, including regression, tests for non-normality and representations of variability. This study also expands on the earlier chapter by including a number of different power train configurations. Included in this study are internal combustion engines (ICEV), electric vehicles (EV), hybrids (HEV), plug-in hybrids (PHEV), as well as fuel cell vehicles (FCV). Also under study in this chapter are the lightweight configurations for all of these different power train vehicles. Lightweight configurations substitute more conventional materials such as steel and cast iron with carbon fibre reinforced plastic (CFRP), glass fibre reinforced plastic (GFRP) and aluminum. Accordingly, light configurations see overall reductions in vehicle weight. The contribution of this study lies in that no specific study has yet to focus on temporality owing to age of data, which has been the subject of concern with LCA practitioners. Indeed, other than Memary et al. 
Table 4.1 An updated list of materials used in Greenhouse gases, Regulatory Emissions, and Energy use in Transportation 2 as of 2012.

\begin{tabular}{|c|c|c|}
\hline Metals & Plastics and Composites & Miscellaneous \\
\hline \multicolumn{3}{|c|}{ Previous Materials } \\
\hline $\begin{array}{l}\text { Cast iron } \\
\text { Copper } \\
\text { Lead } \\
\text { Steel } \\
\text { Recycled steel } \\
\text { Stainless steel } \\
\text { Magnesium } \\
\text { Aluminum } \\
\text { Recycled aluminum }\end{array}$ & $\begin{array}{l}\text { HDPE (high density polyethylene) } \\
\text { PP (polypropylene) } \\
\text { PET (polyethylene terephthalate) } \\
\text { CFRP (carbon fibre reinforced } \\
\text { plastic) } \\
\text { GFRP (glass fibre reinforced plastic) }\end{array}$ & $\begin{array}{l}\text { Rubber } \\
\text { Glass } \\
\text { Paint }\end{array}$ \\
\hline \multicolumn{3}{|c|}{ New Materials } \\
\hline $\begin{array}{l}\text { Platinum } \\
\text { Nickel }\end{array}$ & $\begin{array}{l}\text { ABS (acrylonitrile butadiene styrene) } \\
\text { EPDM (ethylene propylene diene monomer) } \\
\text { Epoxy } \\
\text { GPPS (general purpose polystyrene) } \\
\text { HIPS (high impact polystyrene) } \\
\text { LDPE (low density polyethylene) } \\
\text { LLDPE (linear low density polyethylene) } \\
\text { Nylon } 6 \\
\text { Nylon } 66 \\
\text { PC (polycarbonate) } \\
\text { PUR(F) (flexible polyurethane) } \\
\text { PUR(R) (rigid polyurethane) } \\
\text { PVC (polyvinyl chloride) }\end{array}$ & $\begin{array}{l}\text { PTFE (polytetrafluoroethylene) } \\
\text { PFSA (perfluorosulfonic acid) } \\
\text { PFSA + suspension material } \\
\text { Carbon Paper }\end{array}$ \\
\hline
\end{tabular}

Table 4.2 Weight in kg of individual materials in Greenhouse gases, Regulatory Emissions, and Energy use in Transportation 2.

\begin{tabular}{|c|c|c|c|c|c|c|c|c|c|c|c|c|c|c|}
\hline & & Rubber & Paint & Glass & Cast Iron & Lead & Copper & Steel & Steel (R) & Steel (S) & $\mathrm{Al}$ & $A I(R)$ & $\mathrm{Mg}$ & $\mathrm{Pt}$ \\
\hline \multirow[t]{2}{*}{ ICEV } & Regular & 30.5 & 11.8 & 38.7 & 147.2 & 16.33 & 25.3 & 619.3 & 222.1 & & 35.9 & 56.5 & 0.24 & 0.007 \\
\hline & Light & 24.3 & 5.9 & 25.2 & 29.9 & 16.33 & 25.4 & 192.7 & 69.1 & 9.12 & 67.3 & 126.5 & 2.94 & 0.008 \\
\hline \multirow[t]{2}{*}{ EV } & Regular & 34.0 & 11.8 & 67.3 & 38.6 & 16.33 & 90.7 & 946.5 & 339.5 & & 33.7 & 92.3 & 0.41 & \\
\hline & Light & 32.0 & 5.9 & 43.0 & 34.9 & 16.33 & 73.6 & 187.6 & 67.3 & & 86.4 & 178.7 & 5.37 & \\
\hline \multirow[t]{2}{*}{ HEV } & Regular & 25.5 & 11.8 & 43.0 & 84.3 & 16.33 & 62.4 & 706.5 & 253.4 & & 33.9 & 65.5 & 0.26 & 0.005 \\
\hline & Light & 22.3 & 5.9 & 27.8 & 29.0 & 16.33 & 49.0 & 218.2 & 78.3 & 6.36 & 70.1 & 114.6 & 3.34 & 0.004 \\
\hline \multirow[t]{2}{*}{ PHEV } & Regular & 25.8 & 11.8 & 45.1 & 79.2 & 16.33 & 64.7 & 706.5 & 253.4 & & 33.9 & 65.5 & 0.28 & 0.005 \\
\hline & Light & 22.9 & 5.9 & 28.9 & 30.2 & 16.33 & 50.8 & 222.5 & 79.8 & 5.68 & 66.2 & 119.6 & 3.57 & 0.004 \\
\hline \multirow[t]{2}{*}{ FCV } & Regular & 26.4 & 11.8 & 47.8 & 26.2 & 16.33 & 74.0 & 715.0 & 256.5 & & 81.0 & 54.3 & 0.29 & 0.097 \\
\hline & Light & 24.2 & 5.9 & 31.4 & 23.5 & 16.33 & 59.0 & 180.0 & 64.9 & & 108.4 & 114.8 & 3.92 & 0.075 \\
\hline
\end{tabular}

Table 4.3 Weight in kg of individual plastics used in Greenhouse gases, Regulatory Emissions, and Energy use in Transportation 2.

\begin{tabular}{|c|c|c|c|c|c|c|c|c|c|c|c|c|c|c|c|c|c|c|c|}
\hline & & HDPE & PP & PET & ABS & EPDM & Epoxy & GPPS & HIPS & LDPE & LLDPE & Nylon6 & Nylon66 & PC & \begin{tabular}{l|l} 
PUR(f) \\
\end{tabular} & PUR(R) & PVC & CFRP & GFRP \\
\hline \multirow[t]{2}{*}{ ICEV } & Regular & 2.10 & 27.2 & 2.55 & 11.4 & 10.7 & 16.1 & 1.05 & 1.05 & 2.10 & 2.10 & 1.65 & 10.5 & 5.3 & 18.3 & 17.4 & 20.7 & & \\
\hline & Light & 1.59 & 20.6 & 1.94 & 8.7 & 8.1 & 12.2 & 0.80 & 0.80 & 1.59 & 1.25 & 1.25 & 8.0 & 4.0 & 13.9 & 13.2 & 15.7 & 124.5 & 14.8 \\
\hline \multirow[t]{2}{*}{ EV } & Regular & 3.28 & 42.5 & 3.99 & 17.8 & 16.7 & 25.1 & 1.64 & 1.64 & 3.28 & 3.28 & 2.58 & 16.4 & 8.2 & 28.6 & 27.2 & 32.4 & & \\
\hline & Light & 2.53 & \begin{tabular}{l|l}
32.8 \\
\end{tabular} & 3.08 & 13.8 & 12.9 & 19.4 & 1.27 & $\begin{array}{ll}1.27 \\
\end{array}$ & 2.53 & 2.53 & 1.99 & 12.7 & 6.3 & 22.1 & 21.0 & 25.0 & 254.2 & 31.5 \\
\hline \multirow[t]{2}{*}{ HEV } & Regular & 2.15 & 27.8 & 2.61 & 11.7 & 10.9 & 16.4 & 1.07 & 1.07 & 2.15 & 2.15 & 1.69 & 10.7 & 5.4 & 18.7 & 17.8 & 21.2 & & \\
\hline & Light & 1.60 & 20.7 & 1.94 & 8.7 & 8.1 & 12.2 & 0.80 & 0.80 & 1.60 & 1.60 & 1.26 & 8.0 & 4.0 & 13.9 & 13.3 & 15.8 & 147.3 & 17.6 \\
\hline \multirow[t]{2}{*}{ PHEV } & Regular & 2.15 & 27.8 & 2.61 & 11.7 & 10.9 & 16.4 & 1.07 & 1.07 & 2.15 & 2.15 & 1.69 & 10.7 & 5.4 & 18.7 & 17.8 & 21.2 & & \\
\hline & Light & 1.75 & 22.6 & 2.12 & 9.5 & 8.9 & 13.4 & 0.87 & 0.87 & 1.75 & 1.75 & 1.37 & 8.7 & 4.4 & 15.2 & 14.5 & 17.2 & 163.2 & 19.7 \\
\hline \multirow[t]{2}{*}{ FCV } & Regular & 2.46 & 31.9 & 2.99 & 13.4 & 12.5 & 18.8 & 1.23 & 1.23 & 2.46 & 2.46 & 1.94 & 12.3 & 6.2 & 21.5 & 20.4 & 24.3 & 134.2 & \\
\hline & Light & 2.46 & 31.9 & 2.99 & 13.4 & 12.5 & 18.8 & 1.23 & 1.23 & 2.46 & 2.46 & 1.94 & 12.3 & 6.2 & 21.5 & 20.4 & 24.3 & 288.4 & 21.2 \\
\hline
\end{tabular}


Table 4.4 Weight in kg of materials specific to fuel cell vehicles used in Greenhouse gases, Regulatory Emissions, and Energy use in Transportation 2.

\begin{tabular}{|l|r|r|r|r|r|}
\cline { 2 - 7 } \multicolumn{1}{c|}{} & $\mathrm{Ni}$ & \multicolumn{1}{l|}{ PTFE } & \multicolumn{1}{l|}{ PFSA } & PFSA (S) & \multicolumn{1}{c|}{ C Paper } \\
\hline Regular & 1.30 & 1.51 & 5.58 & 0.65 & 5.45 \\
\hline Light & 1.01 & 1.17 & 4.51 & 0.50 & 4.26 \\
\hline
\end{tabular}

(2012), literature specifically dedicated to time-series data analysis in LCA is lacking. Because the implications of this research have import for one particular aspect of LCA implicated in more robust results, there is cachet in the findings of this study as to how serious a problem this is and what direction should be pursued in moving forward.

\subsection{Methods}

Because a number of different methods are used in concert with each other, this chapter will be broken down into individual sub-chapters, each with their respective subject matter. Figure 4.1 shows the overall layout of this study and, hence, the rationale for the structure and order of the following sections.

\subsubsection{Data collection}

As in with the earlier study, the data employed in this study was taken from publicly available reports (peer-reviewed or government), congresses, proceedings, technical papers, and LCAs (see Appendix A for full references). Also used in concert with these data sources were international databases and corporate sustainability reports (CSR). Among others, this would include public databases such as the Inventory of Carbon and Energy (ICE) from the University of Bath (UK), the Interuniversity Research Centre for the Life Cycle of Products, Processes and Services (CIRAIG) of Canada, SPINE (Sustainable Product Information Network for the Environment) of Sweden, and the Centre for Building Performance Research of New Zealand. Some data from architecture and building science publications was not incorporated as their units of energy were based on the area (footage) of the building under consideration (e.g., $\mathrm{J} / \mathrm{m}^{2}$ ) and, hence, incompatible. Nevertheless, there are 368 sources for the data and 2,824 data points. Collection and consolidation of this data was highly time consuming (more than a year) as it was scattered across many different published sources, a considerable number of which had to be 
discarded as they were not the original source documents for the data. Indeed, the archived databases maintained by the World Steel Association, for example, are very rare. Lastly, with regard to the data used in this study, it should also be stated that certain background processes have relevance to this study (age of data, efficiency gains, power grid make up, etc.) to the exclusion of all

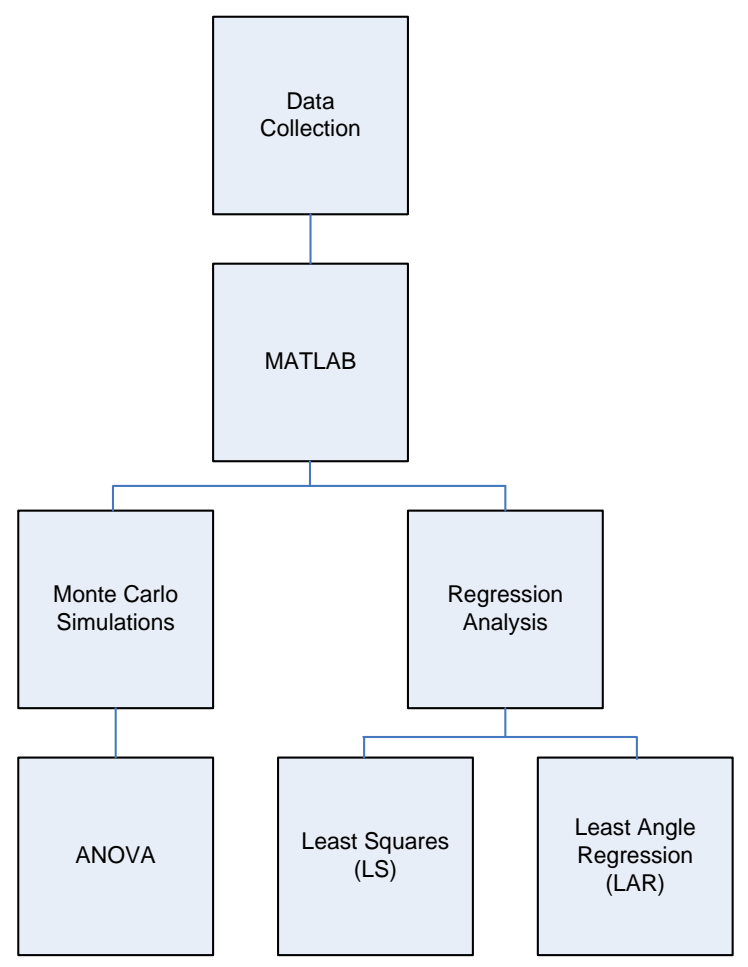

Figure 4.1 The structure of the study in Chapter 4.

others. Background processes are factors which explain some of the differences seen in data measurements of similar processes. They are discussed and assessed in detail as to their relevance in Chapter 3.

\subsubsection{Monte Carlo simulations and analysis of variation}

This study utilizes Monte Carlo simulations to model the effects of temporality across a large time periods (see Table 4.5). Monte Carlo simulations are a suitable method for studying variance and uncertainty in LCA (Hertwich et al., 2000; Björkland, 2002; Heijungs and Frischnecht, 2005). These simulations involve determining a probability distribution for each of 
the inputs (energy intensities of materials in $\mathrm{kWh} / \mathrm{kg}$ in Table 4.1), and using a randomly generated set to recreate the distributions as outputs (Maurice et al., 2000). The Monte Carlo simulations were programmed in MATLAB $2013 \mathrm{~b}$ to $2014 \mathrm{a}$ and utilized $10^{8}$ total iterations. That is, there were 10,000 simulations, each with 10,000 data points. As was found in the earlier study, this was deemed to be sufficiently robust as mean and median values were hardly different and the standard error was very small. MATLAB utilizes a MT19937 Mersenne Twister pseudorandom number generator (PRNG) which has proven to be non-biased (Hongo et al., 2010). The importance of this PRNG is that it does not promote statistically significant error propagation, which is of concern in Monte Carlo simulations. Individual probability distributions were determined using the kernel smoothing function estimate (ksdensity) embedded within MATLAB. Historical data on the energy intensity of each individual material in $\mathrm{kW} / \mathrm{kg}$ (see Table 4.1) was converted into individual cumulative probability distributions on which the randomized set consisting of ten thousand iterations created by the PRNG was fit, thus creating energy intensity probability distributions. Individual material masses (kg) supplied by GREET2 were multiplied by their corresponding energy intensity probability distributions $(\mathrm{kWh} / \mathrm{kg})$. In turn, these individual distributions corresponding to individual materials were added up to get the total energy intensity $(E)$ for a vehicle $(\mathrm{kWh})$. Thus, the total energy per vehicle may be expressed as follows:

$$
E=\sum_{i=1}^{N} m_{i} d_{i}
$$

where $m$ is the mass of the individual material, $d$ is the energy intensity $(\mathrm{kWh} / \mathrm{kg})$, and $N$ is the number of materials in Table 4.1. The individual masses of the materials of manufacture are given in Tables 4.2, 4.3, and 4.4. The time frame that the existing data spans is in Table 4.5 and, it should be noted, these time frames are dissimilar. Other methods employed in this study, such as one way ANOVA (analysis of variance), also used embedded MATLAB software for their analyses (see Figure 4.1 for the overall structure of this study). The results of the ANOVA were presented as box plots in order to visualize the variance in each material. The vertical ends of each of the boxes represent the $25^{\text {th }}$ and $75^{\text {th }}$ percentiles while the whiskers represent 2.7 standard deviations, or 99.3 percent coverage of a normal distribution. 
Table 4.5 Time over which the available data on energy intensity $(\mathrm{kWh} / \mathrm{kg})$ of individual materials spans.

\begin{tabular}{|l|l|l|l|}
\hline Rubber & $1968-2012$ & ABS & $1991-2012$ \\
\hline Paint & $1993-2012$ & EPDM & $1999-2012$ \\
\hline Glass & $1968-2012$ & Epoxy & $1981-2012$ \\
\hline Cast Iron & $1973-2012$ & GPPS & $1979-2013$ \\
\hline Lead & $1973-2012$ & HIPS & $2003-2012$ \\
\hline Copper & $1968-2012$ & HDPE & $1973-2012$ \\
\hline Magnesium & $1983-2012$ & LDPE & $1979-2013$ \\
\hline Steel & $1960-2011$ & LLDPE & $1981-2013$ \\
\hline Steel (R) & $1981-2009$ & PET & $1979-2011$ \\
\hline Aluminum & $1968-2012$ & PP & $1973-2011$ \\
\hline Aluminum (R) & $1983-2009$ & Nylon 6 & $1980-2012$ \\
\hline PTFE & $2000-2012$ & Nylon 66 & $1979-2012$ \\
\hline Platinum & $1979-2011$ & PC & $1980-2012$ \\
\hline PFSA & $2000-2012$ & PUR (flex) & $1991-2012$ \\
\hline PFSA Suspension & $2000-2012$ & PUR (rigid) & $1980-2012$ \\
\hline Carbon paper & $2000-2012$ & PVC & $1980-2013$ \\
\hline Nickel & $1989-2012$ & CFRP & $1990-2013$ \\
\hline & & GFRP & $1983-2012$ \\
\cline { 2 - 4 }
\end{tabular}

Table 4.6 Average energy totals, standard deviations and coefficient of variation for various power train configurations $(\mathrm{kWh})$.

\begin{tabular}{|c|c|c|c|c|}
\hline & \multirow{2}{*}{\begin{tabular}{|l|} 
Mean \\
\end{tabular}} & \multirow[b]{2}{*}{ SD } & \multirow{3}{*}{\begin{tabular}{|l|}
\multicolumn{2}{|l|}{ CV } \\
\end{tabular}} \\
\hline & & & & \\
\hline ICEV & Regular & 11,719 & 6,420 & \\
\hline & Light & 8,938 & 4,326 & 0.484 \\
\hline \multirow[t]{2}{*}{ HEV } & Regular & 12,491 & 7,107 & 0.569 \\
\hline & Light & 9,389 & 4,553 & 0.485 \\
\hline \multirow[t]{2}{*}{ PHEV } & Regular & 12,494 & 7,119 & 0.570 \\
\hline & Light & 9,804 & 4,730 & 0.482 \\
\hline \multirow[t]{2}{*}{ EV } & Regular & 16,788 & 9,496 & 0.566 \\
\hline & Light & 12,970 & 5,998 & 0.462 \\
\hline \multirow[t]{2}{*}{ FCV } & Regular & 14,029 & 7,459 & 0.532 \\
\hline & Light & 11,978 & 5,102 & 0.426 \\
\hline
\end{tabular}




\subsubsection{Regression analysis}

Regression analysis was utilized in the previous study, although it was merely used in an ancillary fashion to illustrate certain phenomena which led to suggestions regarding methods. Specifically, a simple least squared (LS) method was used to show the unfeasibility of using small time periods of five years as a basis for comparison of variation with other time intervals. LS regression solves the first order linear equation:

$$
y=p_{1} x+p_{2}
$$

by solving for the following coefficients:

$$
p_{1}=\frac{n \sum x_{i} y_{i}-\sum x_{i} \sum y_{i}}{n \sum x_{i}^{2}-\sum\left(x_{i}\right)^{2}}
$$

and,

$$
p_{2}=\frac{1}{n}\left(\sum y_{i}-p_{1} \sum x_{i}\right)
$$

In this instance, $n$ corresponds to the number of data points. This method was used in the previous study, although it was recommended for the furtherance of study in this area that the least absolute residuals (LAR) be used. Simply put, in $n-1$ steps, LAR regression finds a fit such that it minimizes the residuals $\left(r_{i}\right)$ as given by:

$$
r_{i}=\left\|y_{i}-\hat{y}_{i}\right\|
$$

where $y_{i}$ is the data and $\hat{y}_{i}$ is the fit (Mathworks, 2015). In using this method, a more robust fit is realized by the fact that the extreme values have lesser influence in finding the fit. LAR can also be used for higher order polynomials, although no better fit was found when using higher order polynomials. Hence, in order to provide a basis of comparison with LS, a first order polynomial was used with the LAR. The rationale behind the use of LAR lies in that the trend identified by the slope of the regression fit $\left(p_{1}\right)$ in LS would be highly influenced by outliers or extreme values, as is immediately apparent in the above formulae since each data point figures in the 
calculation of the above coefficients. However, as discussed in the Results chapter, this has no bearing on the derivation of percentage increases of energy intensity $(\mathrm{kWh} / \mathrm{kg})$, but is purposeful in identifying or corroborating that observations of variance are not simply due to scattered raw data.

Table 4.12 summarizes the results of both regression analyses. However, an explanation of the various statistics utilized should be made at this point. The $+/$ - columns in Table 4.12 indicate the $95 \%$ confidence interval for the corresponding coefficients $p_{1}$ and $p_{2}$. The R-square $\left(R^{2}\right)$ measure explains how well the fit of the data is explained by its variation. The R-square value normally ranges between 0 to 1 and can be expressed as a percentage. Consequently, an Rsquare value of 0.95 or $95 \%$ means that $95 \%$ of the data explains the variation in the data about the mean. Thus, the closer the value gets to 1 or $100 \%$, the more the proportion of the variance is accounted for in the model. R-square is commonly expressed as follows:

$$
R^{2}=1-\frac{S S E}{S S T}
$$

where $S S E$ is the sum of the squares for error and SST is the sum of the squares about the mean. In turn, each of these may be expressed such that:

$$
S S T=\sum n_{j}\left(x_{j}-\bar{x}\right)^{2}
$$

and

$$
S S E=\sum\left(n_{j}-1\right) s_{j}^{2}
$$

where $n_{j}=$ size of sample from population $j$

$\bar{x}=$ mean of all $\mathrm{n}$ observations

$\bar{x}_{j}=$ mean of sample from population $j$

$s_{j}^{2}=$ variance of sample from population $j$

A negative R-square value is possible when there is no constant term in the equation (i.e., $p_{2}$ ) and such cases suggest the addition of a constant term (Mathworks, 2015). The root mean squared 
error $(R M S E)$ is a measure of the standard error of the regression. As such, it is a measure of the standard deviation of the random component of the data such that a value closer to 0 gives an indication of a fit which is more useful for prediction. Hence, the RMSE can be expressed in terms of the mean squared error (MSE) and, consequently, it can also be expressed in terms of the squares for error $(S S E)$ :

$$
R M S E=\sqrt{M S E}=\sqrt{\frac{S S E}{n-k}}
$$

where $k=$ number of fitted coefficients

$n=$ total number of observations

Naturally, with greater amounts of data, the more the RMSE tends towards zero.

\subsubsection{Skewness and kurtosis}

As with the previous chapter, this work looks at the skewness and kurtosis of distributions in order to determine whether the data is non-normal or, stated another way, shows logarithmic qualities. The test for this requires the use of log transformations; that is, the logarithm (base 10) of the data is taken and changes in skewness and kurtosis are measured. Skewness can be understood as the degree to which a distribution is symmetrical. Non-symmetrical distributions tend to deviate away from normal distributions, where normal distributions are assigned a value of zero. Kurtosis is the measurement of the flatness of a distribution, again with the normal distribution at a value of three. Hence, a kurtosis greater than three is narrower that the normal distribution and less than three is more flat than a normal distribution. Both skewness and kurtosis are embedded functions within MATLAB and are calculated using the following formulae:

$$
\begin{aligned}
& \text { skewness }=\frac{\sum_{i=1}^{n}\left(x_{i}-\bar{x}\right)^{3} / n}{\sigma^{3}} \\
& \text { kurtosis }=\frac{\sum_{i=1}^{n}\left(x_{i}-\bar{x}\right)^{4} / n}{\sigma^{4}}
\end{aligned}
$$


where $\bar{x}$ is the mean and $\sigma$ the standard deviation. Also, $n$ denotes the number of measurements in this distribution, which in this case is ten thousand.

\subsubsection{Limitations}

As in the earlier chapter, energy intensities for primary processes of the metals were used to the exclusion of energy intensities of secondary processes as there are far too many secondary processes available in order to make baseline comparisons. Energy intensity of primary processes consists of the energy required to take raw materials through to the smelting process, while the energy intensity of secondary processes (usually much less than primary) consists of finishing processes. Hence, all secondary processes such as casting, rolling or extruding were ignored. However, in that secondary energy intensities were ignored, this was applicable only for steel, aluminum and copper. Also, this study focuses strictly on sedan models and, unlike the last study, does not include sport utility vehicles (SUVs) and pick-up trucks (PUTs). Alternatively, this study expands beyond just the ICEV power train to include HEVs, PHEVs, EVs and FCVs. A limitation of the study is a lack of extensive data on key elements of the power train materials for FCVs. This subject is explained further in the next section. Another limitation of this study lies in that it does not purport to answer where temporality due to the age of data arises from, be it technology, efficiency gains, the make-up of national power grids, and so forth.

There are also limitations arising from the use of the GREET2 model. These include the lack of cross-correlation with other existing vehicular models of manufacture and LCA studies of vehicles, although there were no other publicly available LCAs of this kind four years ago at the start of this study. Nevertheless, because of these factors, this study exists as a stand-alone piece. Many of the available software platforms in LCA were not utilized in this study owing to the limited ability to assess their functionality from available literature and demo software versions. For example, very little information exists of the GaBi platform's Monte Carlo simulations. Similarly, SimaPro's literature indicates that their Monte Carlo simulations only allow for four types of probability distribution: normal, lognormal, square, and triangular (Goedkoop et al, 
2013). Hence, SimaPro does not allow for the construction of individual probability distributions from raw data. SimaPro also does not have the ability to test for non-normal distributions. These were key reasons in the decision to use MATLAB. Lastly, the direct measurement skewness and kurtosis to test for non-normal distributions was not the only option available. Other tests for non-normality include the Jarque-Bera test and the D'Agostino's Ksquared test. However, since both of these tests utilize measurements of both skewness and kurtosis, there was deemed to be little added value in introducing these further measurements. Also, neither one of these tests give any insights into whether log transforming distributions will bring about greater clarity.

\subsection{Results and Discussion}

\subsubsection{Monte Carlo simulations and analysis of variance}

Monte Carlo simulations were programmed for the individual materials of manufacture which make up each of the individual power train configurations (ICEV, HEV, PHEV, EV and FCV). Also, a separate set of Monte Carlo simulations was programmed for lightweight configurations of the above power trains. The sum contributions of each of the materials towards total energy per vehicle were then tallied up. Results from all the Monte Carlo simulations are in Table 4.6 in terms of individual vehicles (functional units). It is apparent that the larger the total energy sums found, the larger the corresponding standard deviation. However, it may be seen that the coefficient of variation $(\mathrm{CV})$ for regular vehicles and lightweight vehicles ranges between 0.531 to 0.568 and 0.434 to 0.491 , respectively. Thus, it would seem that the vehicles with more traditional materials (steel, cast iron) experience the effects of variance more than they do for the lightweight vehicles, which use more aluminum than regular vehicles. This is evidenced by the similar energy totals for regular ICEV $(11,659 \mathrm{kWh})$ and light FCV $(11,889 \mathrm{kWh})$, yet their corresponding $C V s$ are 0.537 and 0.434 , respectively. Thus, the reasons for these differences in $C V s$ are owing to the sum total of individual contributions as per each of the raw materials in both the regular and lightweight models. A discussion further to this subject is in the regression 


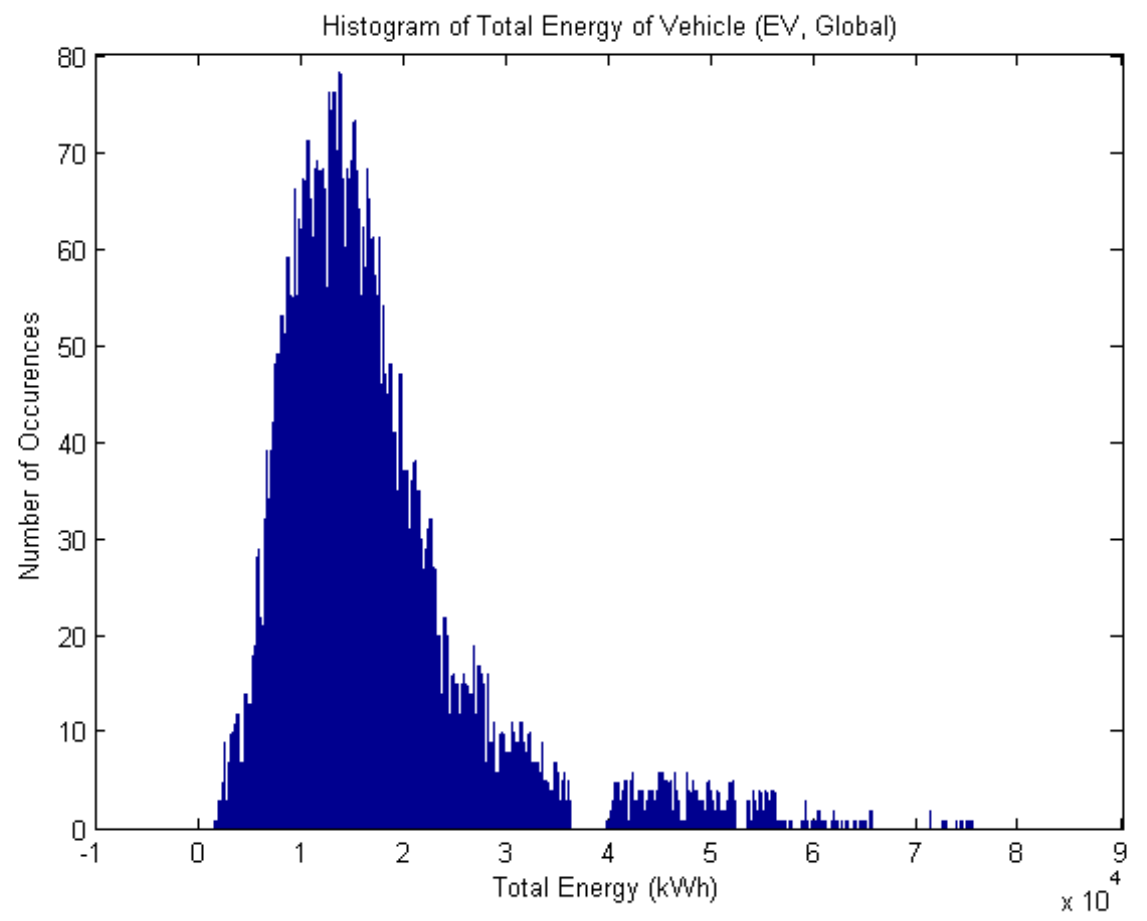

Figure 4.2 An example Monte Carlo distribution for an all electric vehicle.

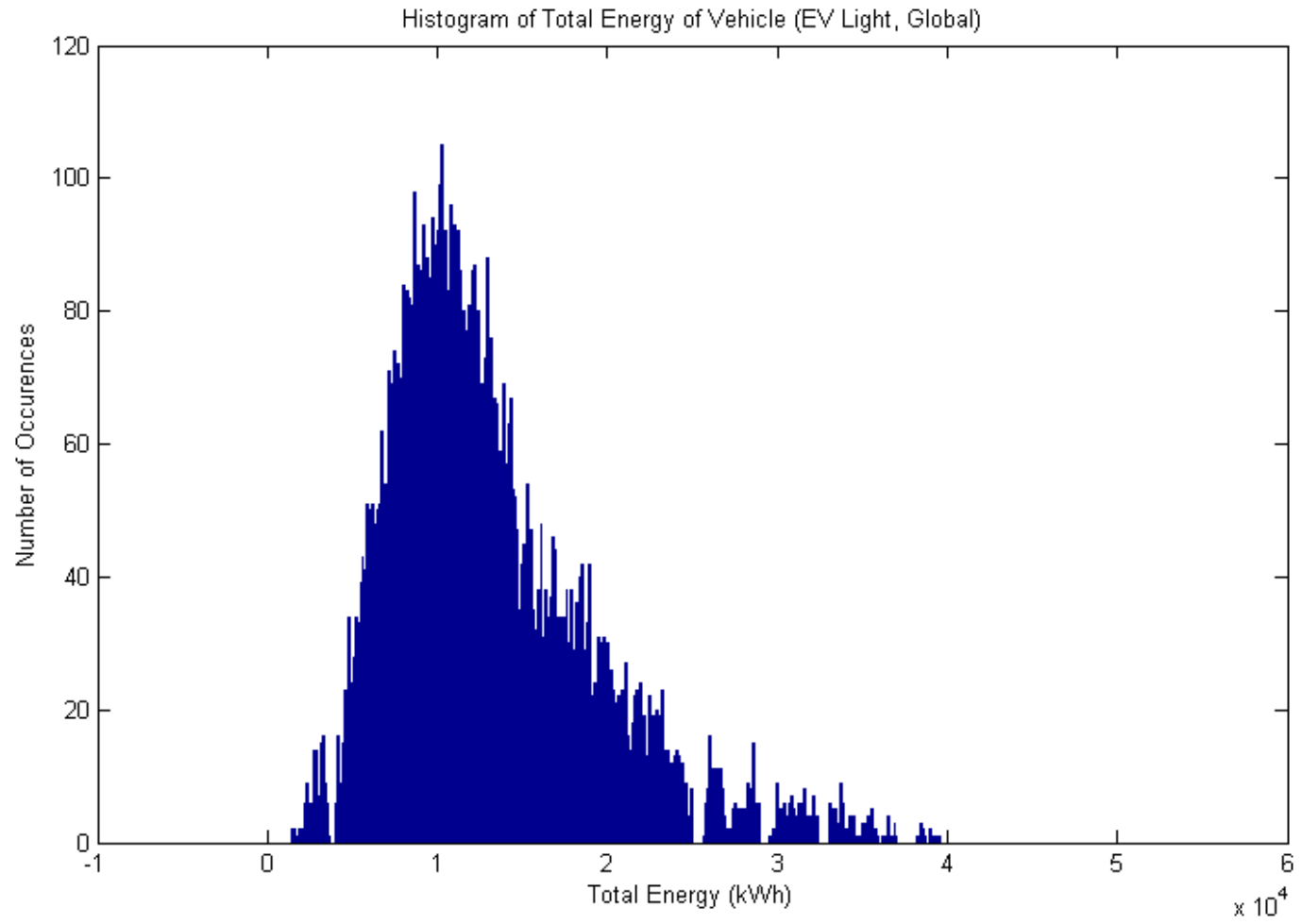

Figure 4.3 An example Monte Carlo distribution for a lightweight all electric vehicle. 
analysis below. The total power for lightweight configurations was less than regular materials, which is surprising since both CFRP and GFRP have high energy intensities of manufacture. However, this may be explained by the heavy usage of recycled aluminum versus virgin aluminum modeled in the GREET2 model, a fact which sees the amount of energy reduced roughly to one tenth. Nevertheless, as mentioned above, only the ICEV power train was previously studied. Thus, it was necessary to examine other power train configurations to make certain that nothing was anomalous in the GREET2 ICEV model specifications which may have influenced the outcomes of the study.

A characteristic Monte Carlo distribution can be seen in Figure 4.2, which is the total energy for regular materials configurations, and in Figure 4.3 for the lightweight configurations. A visible skewness towards the vertical axis (left) can be seen in both Figures. It should be noted here that the choice of an EV power train for Figures 4.2 and 4.3 does not imply any significance in light of the other power trains under study and their corresponding findings. In other words, the EV figures are merely representative examples (see below for other power trains). The subsequent one way ANOVA analyses (energy intensity versus time) showed that in conventional material vehicles the main contributor to the variance was virgin steel followed by recycled steel, then plastics and finally recycled aluminum (see Figures 4.4 and 4.5). With lightweight configurations, the highest contributor to variance was recycled aluminum, followed by virgin steel, plastics and then recycled steel (see Figures 4.6 and 4.7). In the lightweight HEV, PHEV, EV and FCV configurations, copper was a close fifth overall. Also surprising was that the main plastics contributors to variance were the same across all different material and power train configurations. They ranked PP first, followed by Epoxy, PUR(F), PVC, EPDM, PUR(R), Nylon66 and, lastly, ABS. Individual calculations of energy for each material can be seen in Tables 4.7, 4.8, and 4.9. It will be noted that steel, recycled steel, aluminum, and recycled aluminum make up the bulk of the vehicle's energy. Plastics generally make up only a fraction of a vehicle's total energy, but it is a significant portion. For regular material vehicles, this can range between $30.8 \%$ and $35.0 \%$ while, for lightweight material vehicles, this can go from $38.2 \%$ to $47.1 \%$. 


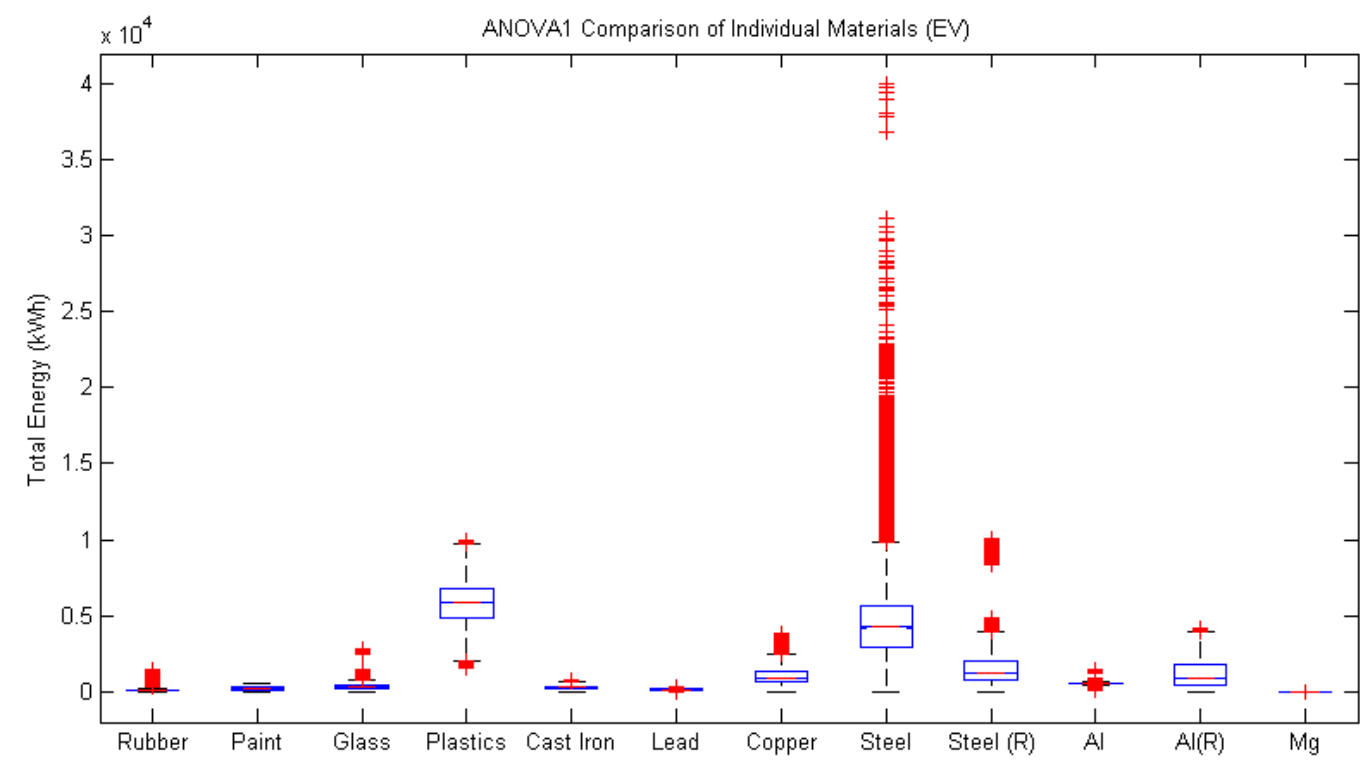

Figure 4.4 Box plots of an analysis of variance results for an all electric vehicle.

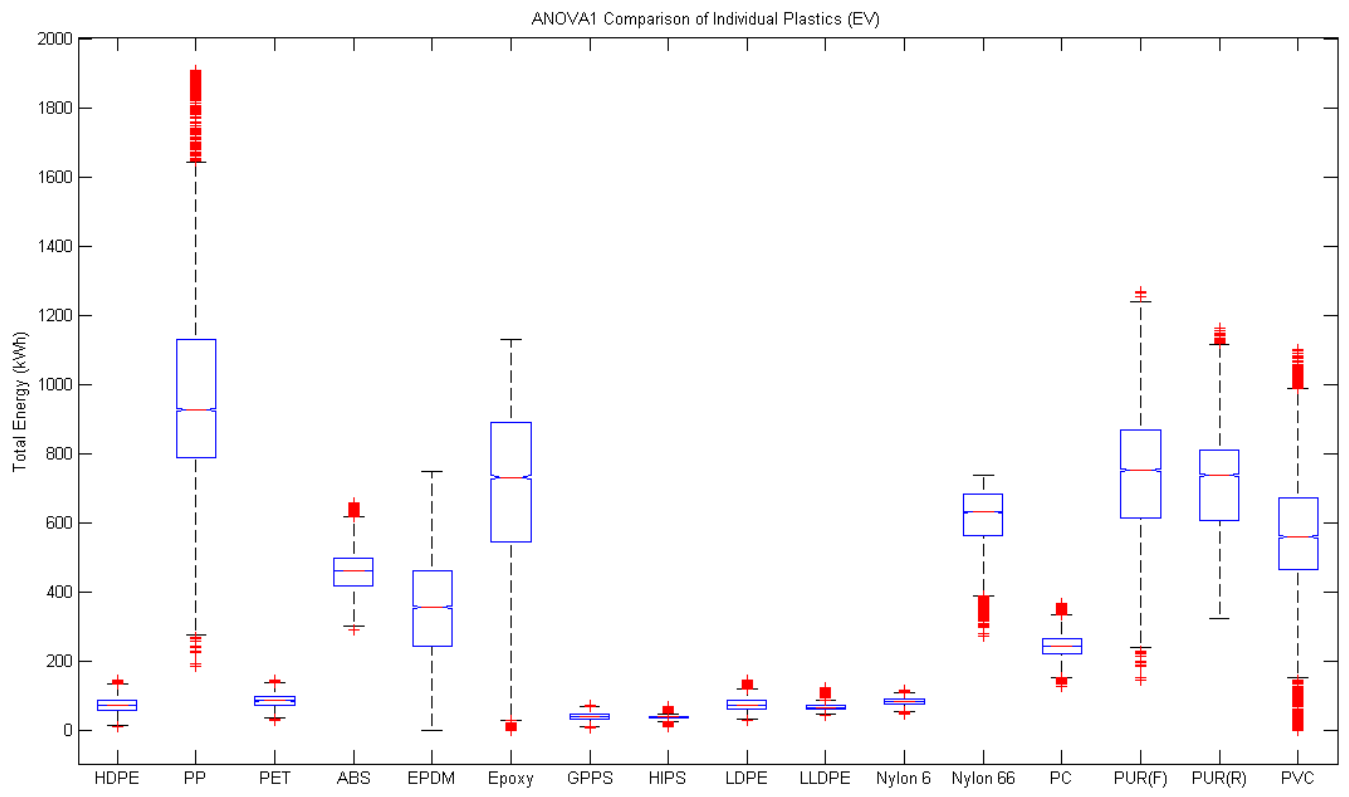

Figure 4.5 Box plots of an analysis of variance for an all electric vehicle (plastics only). 


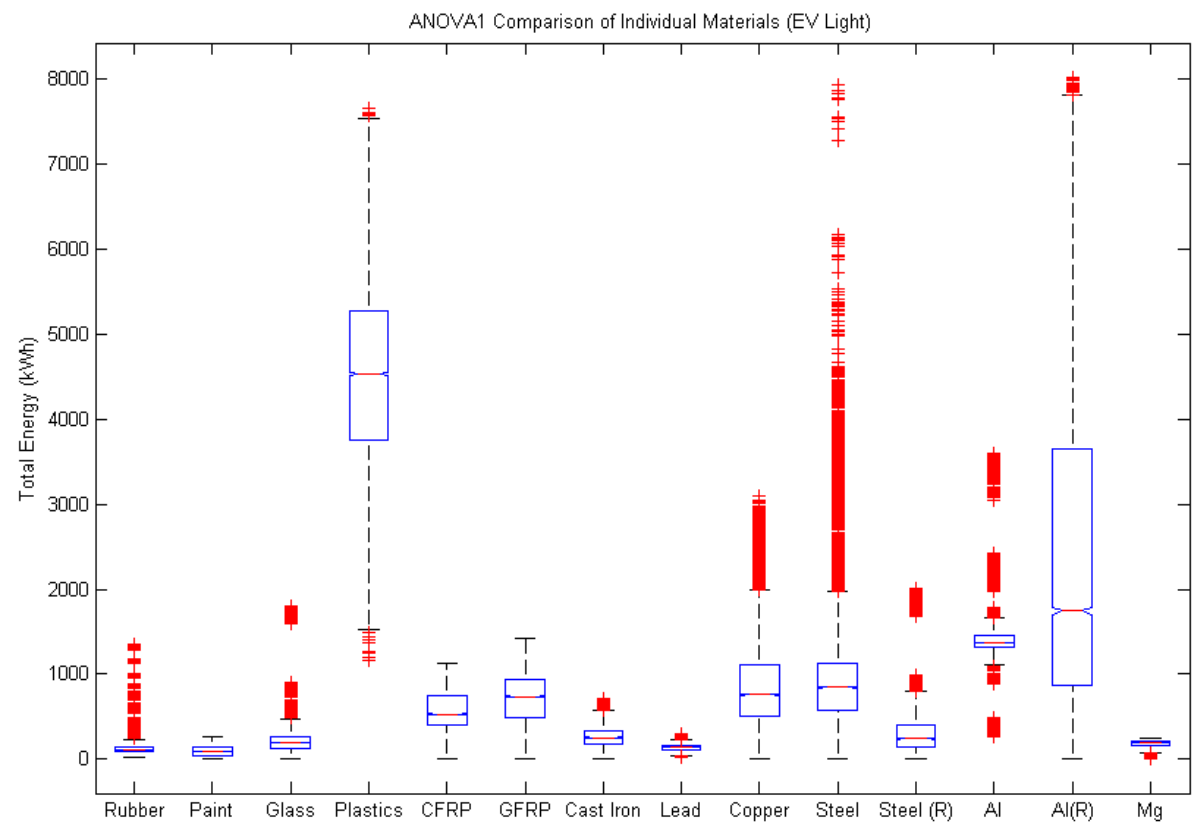

Figure 4.6 Box plots of an analysis of variance for a lightweight all electric vehicle.

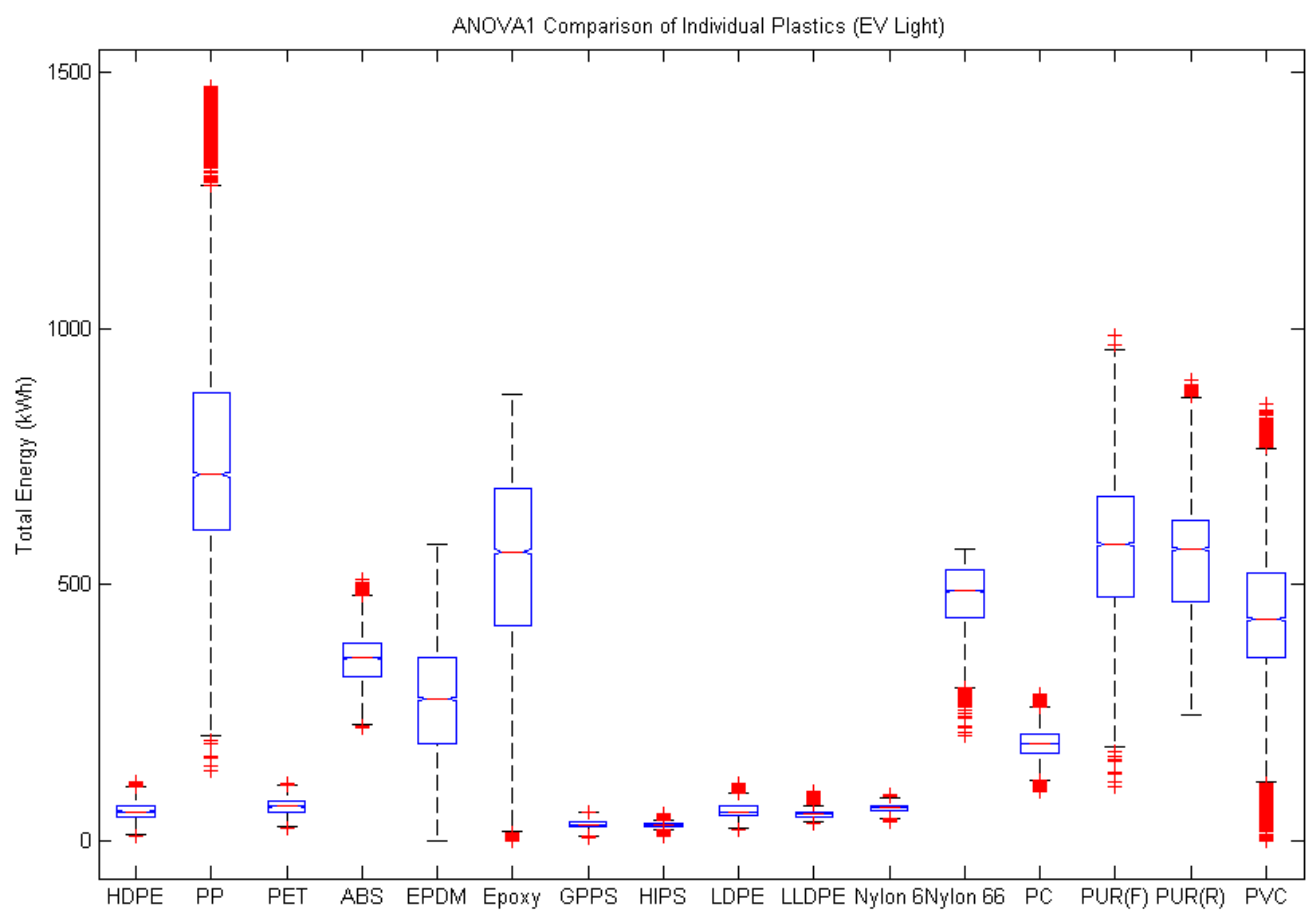

Figure 4.7 Box plots of an analysis of variance for a lightweight all electric vehicle (plastics only). 
Table 4.7 Means and standard deviations of materials for regular and lightweight sedans with different power trains $(\mathrm{kWh})$.

\begin{tabular}{|c|c|c|c|c|c|c|c|c|c|c|c|c|c|c|c|}
\hline & & & Rubber & Paint & Glass & Cast Iron & Lead & Copper & Steel & Steel (R) & Steel (S) & $\mathrm{Al}$ & $\mathrm{Al}(\mathrm{R})$ & $\mathrm{Mg}$ & $\mathrm{Pt}$ \\
\hline \multirow[t]{4}{*}{ ICEV } & Regular & Mean & \begin{tabular}{|r|}
181.0 \\
\end{tabular} & 202.7 & \begin{tabular}{|r|}
201.1 \\
\end{tabular} & \begin{tabular}{|l|}
1109.8 \\
\end{tabular} & 141.8 & 309.8 & $\begin{array}{r}3309.0 \\
\end{array}$ & 1150.9 & & \begin{tabular}{|r|}
591.7 \\
\end{tabular} & 753.0 & 8.2 & 0.20 \\
\hline & & SD & 233.9 & 128.5 & 177.8 & 480.3 & 43.3 & 194.7 & 2558.0 & 1214.6 & & 145.9 & 611.0 & 1.8 & 0.08 \\
\hline & Light & Mean & 144.4 & 101.3 & 130.6 & 225.8 & 141.8 & 311.2 & 1029.5 & 358.1 & 134.8 & 1110.6 & 1686.5 & 101.0 & 0.22 \\
\hline & & SD & 186.6 & 64.2 & 115.5 & 97.7 & 43.3 & 195.6 & 795.8 & 377.9 & 83.9 & 273.9 & 1368.5 & 21.6 & 0.09 \\
\hline \multirow[t]{4}{*}{ EV } & Regular & Mean & 202.1 & 202.7 & 349.5 & 291.2 & 141.8 & 1111.5 & 5056.9 & 1758.9 & & 556.9 & 1230.4 & 14.2 & \\
\hline & & SD & 261.2 & 128.5 & 309.1 & 126.0 & 43.3 & 698.6 & 3909.2 & 1856.2 & & 137.3 & 998.4 & 3.0 & \\
\hline & Light & Mean & 190.1 & 101.3 & 223.0 & 263.1 & 141.8 & 902.1 & 775.0 & 348.7 & & 1426.5 & 2382.0 & 184.4 & \\
\hline & & SD & 245.7 & 64.2 & 197.2 & 113.9 & 43.3 & 567.1 & 348.7 & 368.0 & & 351.8 & 1932.9 & 39.5 & \\
\hline \multirow[t]{4}{*}{ HEV } & Regular & Mean & 151.4 & 202.7 & 223.2 & 635.5 & 141.8 & 764.9 & 3774.9 & 1313.0 & & 559.8 & 873.6 & 9.1 & 0.14 \\
\hline & & SD & 195.6 & 128.5 & 197.3 & 275.0 & 43.3 & 480.8 & 2918.1 & 1385.6 & & 138.1 & 708.9 & 1.9 & 0.06 \\
\hline & Light & Mean & 132.6 & 101.3 & 144.4 & 218.5 & 141.8 & 601.1 & 1165.8 & 405.5 & 94.0 & 1157.7 & 1526.9 & 114.7 & 0.11 \\
\hline & & SD & 171.3 & 64.2 & 127.7 & 94.5 & 43.3 & 377.8 & 901.2 & 427.9 & 58.6 & 285.5 & 1239.0 & 24.5 & 0.05 \\
\hline \multirow[t]{4}{*}{ PHEV } & Regular & Mean & 153.4 & 202.7 & 234.0 & 597.1 & 141.8 & 792.9 & 3774.9 & 1313.0 & & 559.8 & 873.6 & 9.5 & 0.13 \\
\hline & & SD & 198.3 & 128.5 & 206.9 & 258.4 & 43.3 & 498.4 & 2918.1 & 1385.6 & & 138.1 & 708.9 & 2.0 & 0.06 \\
\hline & Light & Mean & 135.8 & 101.3 & 150.1 & 228.0 & 141.8 & 622.7 & 1188.6 & 413.5 & 84.0 & 1093.2 & 1593.3 & 122.4 & 0.11 \\
\hline & & SD & 175.4 & 64.2 & 132.7 & 98.7 & 43.3 & 391.4 & 918.8 & 436.3 & 52.3 & 269.6 & 1293.0 & 26.2 & 0.05 \\
\hline \multirow[t]{4}{*}{ FCV } & Regular & Mean & 156.7 & 202.7 & 248.2 & 197.9 & 141.8 & 907.2 & 3820.0 & 1328.7 & & 1337.2 & 723.3 & 10.1 & 2.76 \\
\hline & & SD & 202.4 & 128.5 & 219.5 & 85.6 & 43.3 & 570.2 & 2953.0 & 1402.2 & & 329.8 & 586.9 & 2.2 & 1.16 \\
\hline & Light & Mean & 144.0 & 101.3 & 162.8 & 177.0 & 141.8 & 722.8 & 962.0 & 336.0 & & 1790.4 & 1529.8 & 134.6 & 2.76 \\
\hline & & SD & 186.1 & 64.2 & 143.9 & 76.6 & 43.3 & 454.3 & 743.6 & 354.6 & & 441.5 & 1241.4 & 28.8 & 1.16 \\
\hline
\end{tabular}

Table 4.8 Means and standard deviations of materials for regular and lightweight sedans with different power trains $(\mathrm{kWh})$.

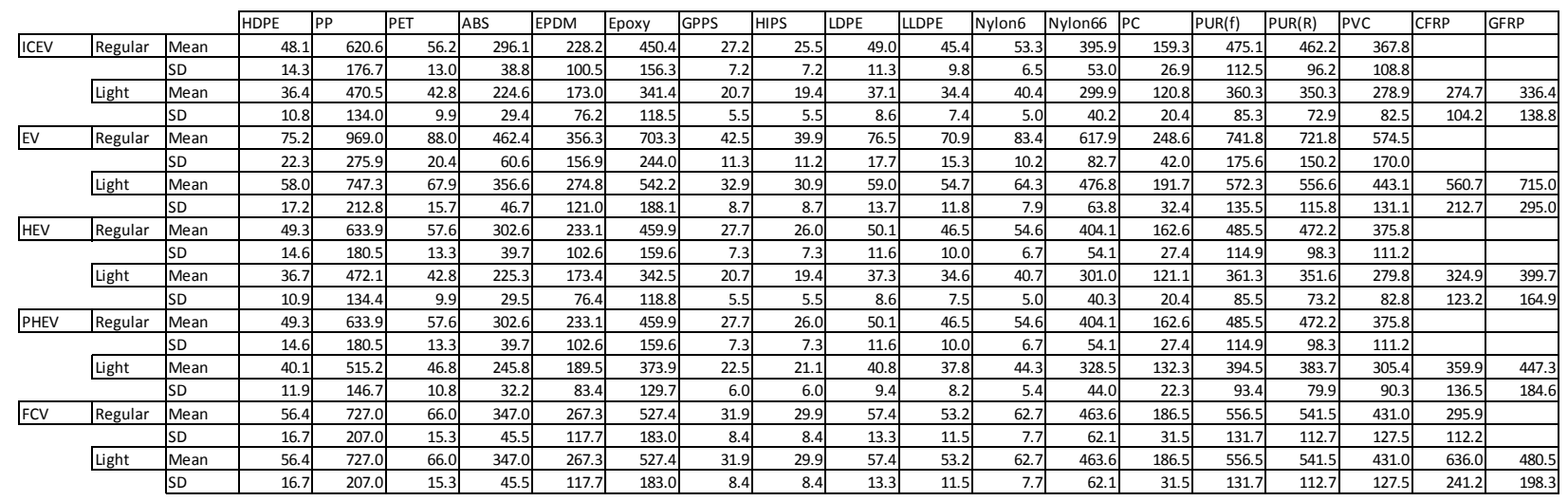

Table 4.9 Means and standard deviations of fuel cell specific materials for regular and lightweight sedans (kWh).

\begin{tabular}{|l|l|r|r|r|r|r|}
\cline { 3 - 7 } \multicolumn{2}{c|}{} & Ni & PTFE & \multicolumn{1}{l|}{ PFSA } & PFSA (s) & C Paper \\
\hline Regular & Mean & 44.1 & 46.6 & 27.5 & 3.1 & 130.4 \\
\hline & SD & 10.7 & 13.1 & 9.0 & 1.0 & 70.5 \\
\hline Light & Mean & 44.1 & 46.6 & 27.5 & 3.1 & 130.4 \\
\hline & SD & 10.7 & 13.1 & 9.0 & 1.0 & 70.5 \\
\cline { 2 - 7 }
\end{tabular}


For the other drive trains, the analysis of variance can be seen in Figures 4.8 through 4.25. A complete breakdown of these figures is as follows:

$\begin{array}{ll}\text { ICEV } & \text { Figures 4.8, 4.9 } \\ \text { ICEV (lightweight) } & \text { Figures 4.10, 4.11 } \\ \text { HEV } & \text { Figures 4.12, 4.13 } \\ \text { HEV (lightweight) } & \text { Figures 4.14, 4.15 } \\ \text { PHEV } & \text { Figures 4.16, 4.17 } \\ \text { PHEV (lightweight) } & \text { Figures 4.18, 4.19 } \\ \text { FCV } & \text { Figures 4.20, 4.21, 4.22 } \\ \text { FCV (lightweight) } & \text { Figures 4.23, 4.24, 4.25 }\end{array}$

As with the previous EV box plot figures, the first figure (e.g., Figure 4.8) gives a visual representation of all the materials in each vehicle, with all the plastics consolidated into one box. The second figure (e.g., Figure 4.9) gives a breakdown of all the plastics. The additional third figures for the FCVs (Figures 4.22 and 4.25) are fuel cell vehicle specific materials. It may be seen with Figures 4.22 and 4.25 that fuel cell specific materials do not significantly contribute towards total vehicle energy.

One recurring theme with regular material vehicles (Figures 4.8, 4.12, 4.16, and 4.20) is to see the predominant variance in the same materials: steel, recycled steel, recycled aluminum, and the plastics. Within the plastics group (Figures 4.9, 4.13, 4.17, and 4.21), most of the variance is with PP, EPDM, epoxy, Nylon 66, the PURs, and PVC. However, continuing with the plastics, this same trend of variance is evident in the lightweight models as well (Figures 4.11, 4.15, 4.19, and 4.24). Nevertheless, comparing regular material vehicles with lightweight vehicles does yield some differences. In regular vehicles, the variance in plastics is similar to the variance of steel. However, in lightweight vehicles variance of plastics exceeds that of steel. Naturally, this is due to the shift in terms of weight in lightweight vehicles to the use of more aluminum, which has the predominant amount of variance (Figures 4.10, 4.14, 4.18, and 4.23). 


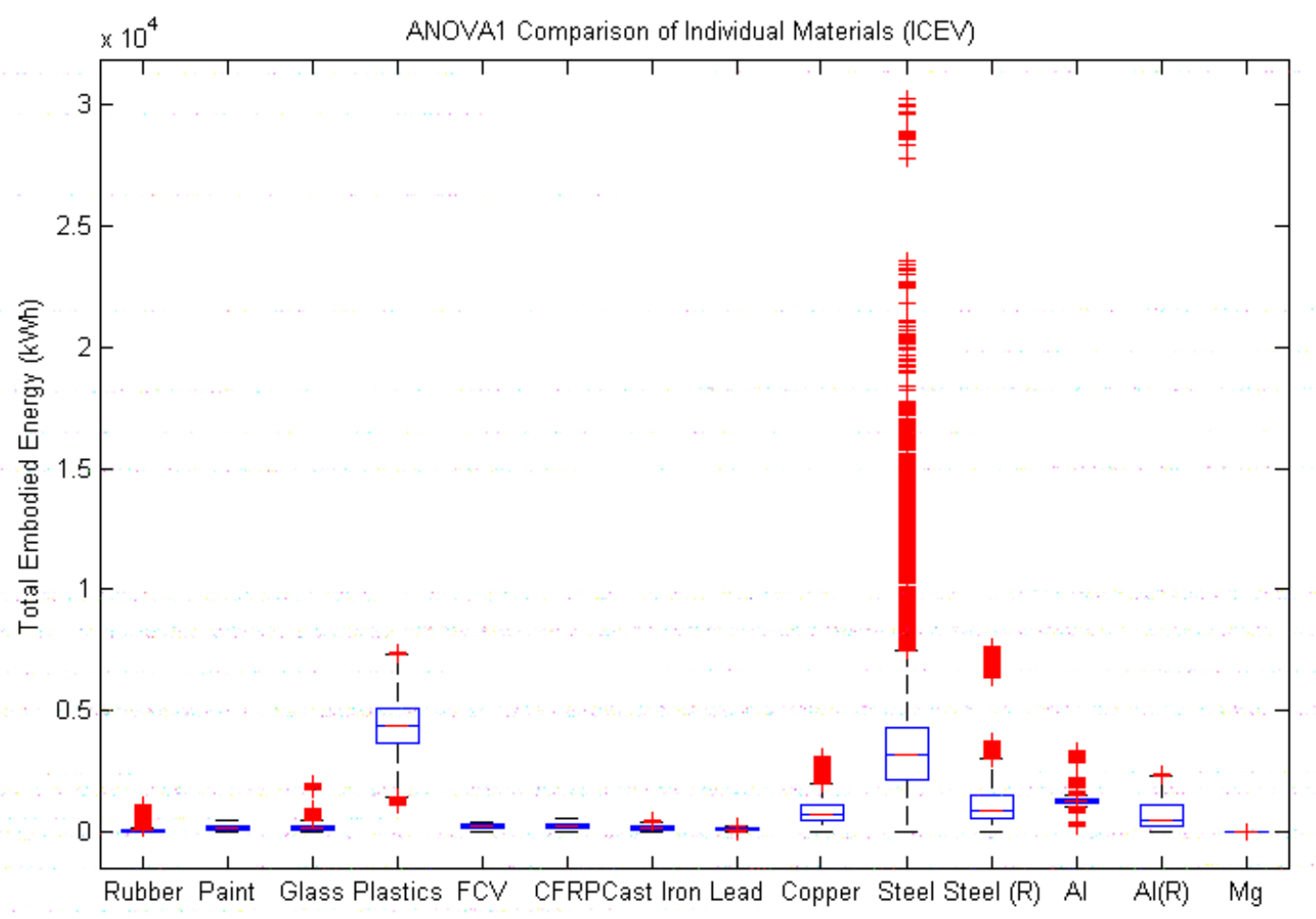

Figure 4.8 Box plots of an analysis of variance results for an internal combustion engine vehicle.

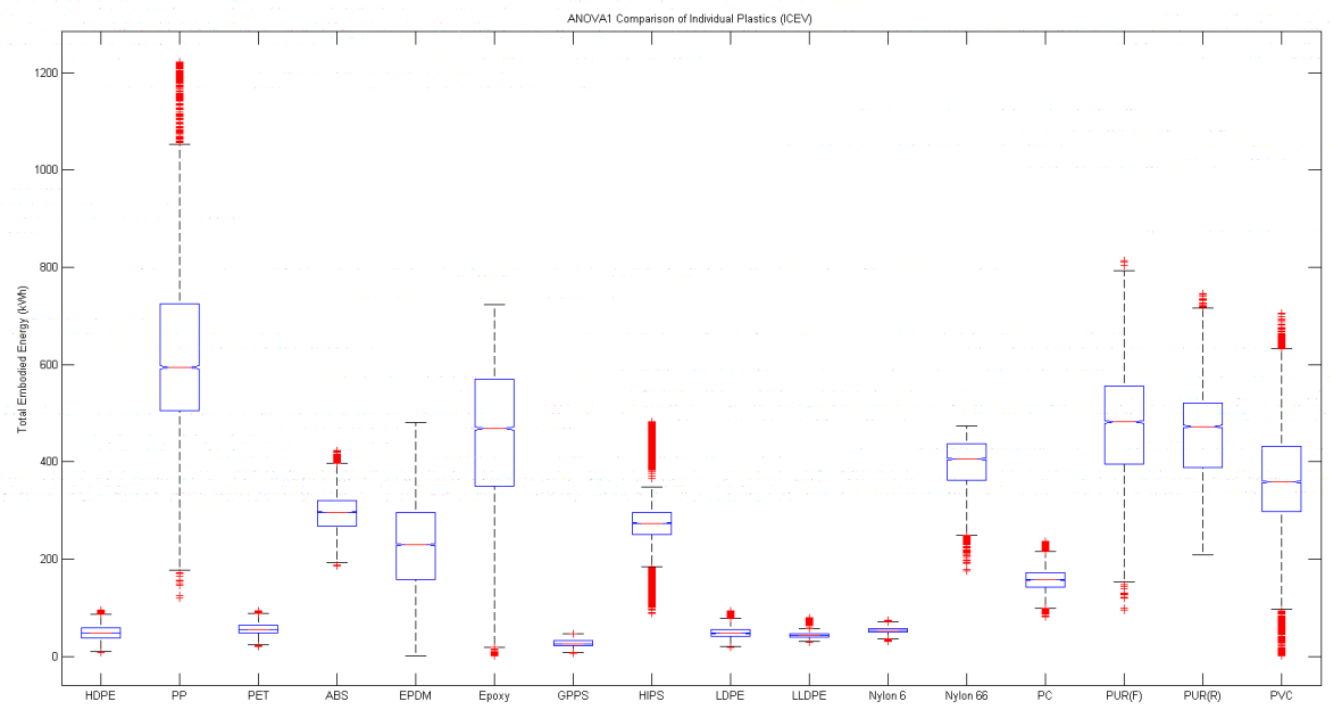

Figure 4.9 Box plots of an analysis of variances results for an internal combustion engine vehicle (plastics only). 


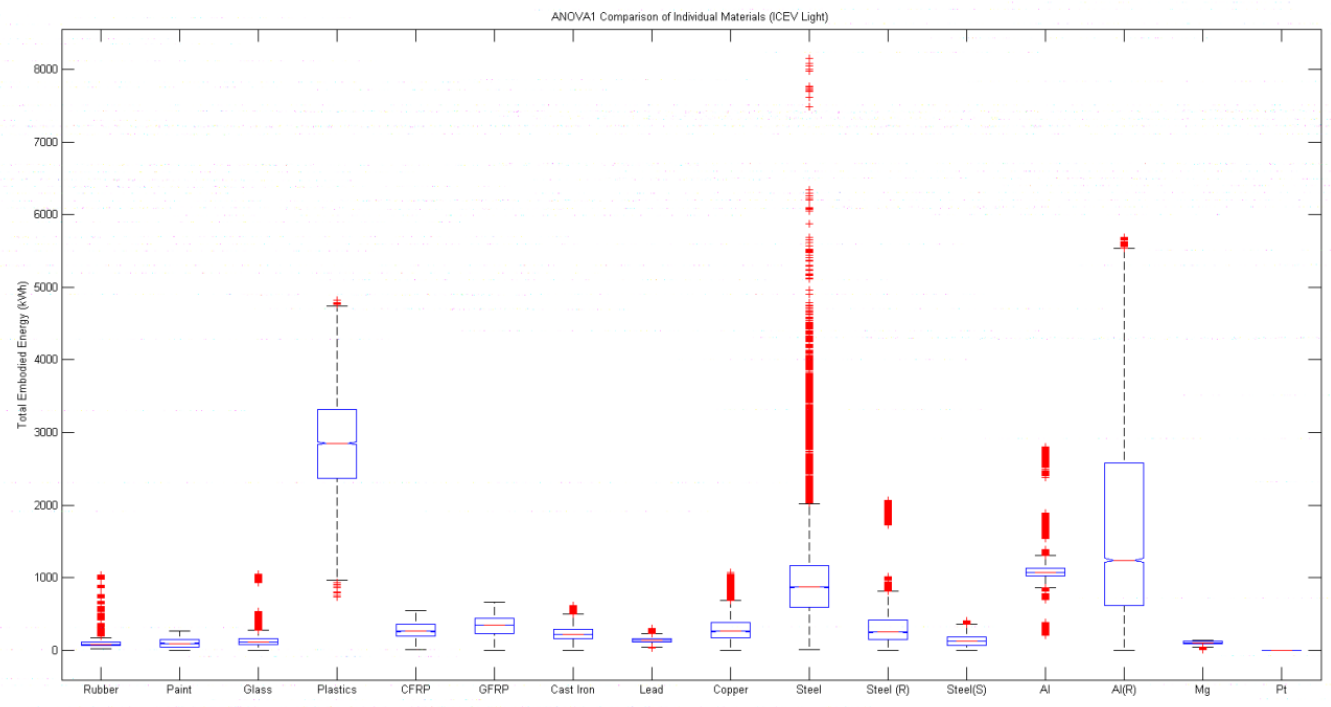

Figure 4.10 Box plots of an analysis of variance results for a lightweight internal combustion engine vehicle.

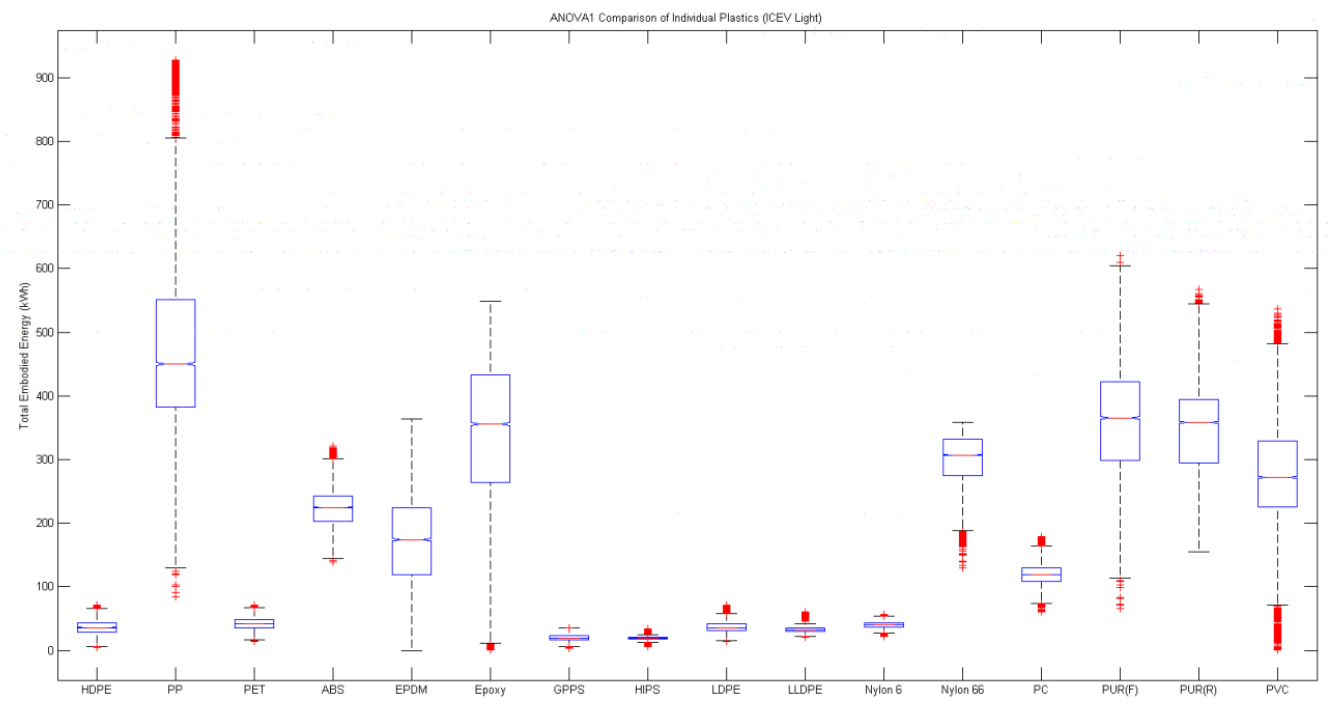

Figure 4.11 Box plots of an analysis of variance results for a lightweight internal combustion engine vehicle (plastics only). 


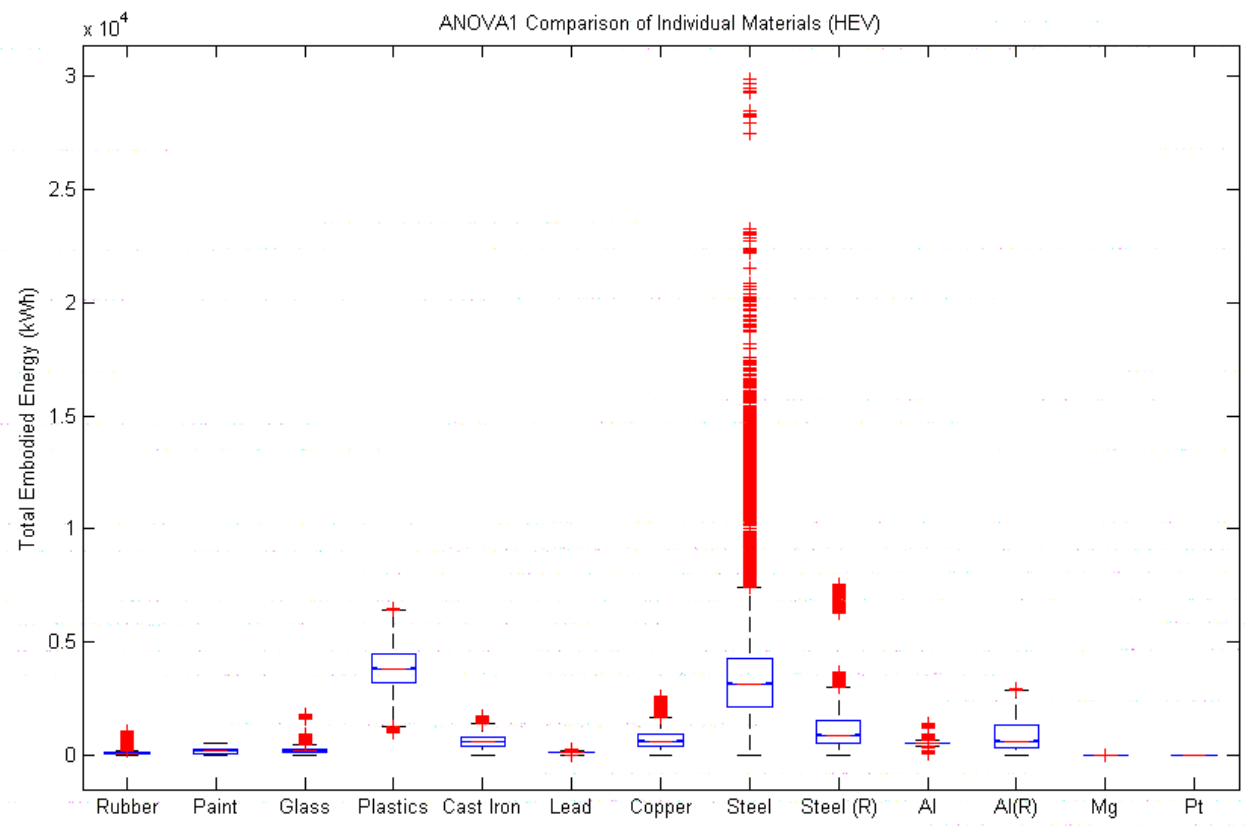

Figure 4.12 Box plots of an analysis of variance results for a hybrid electric vehicle.

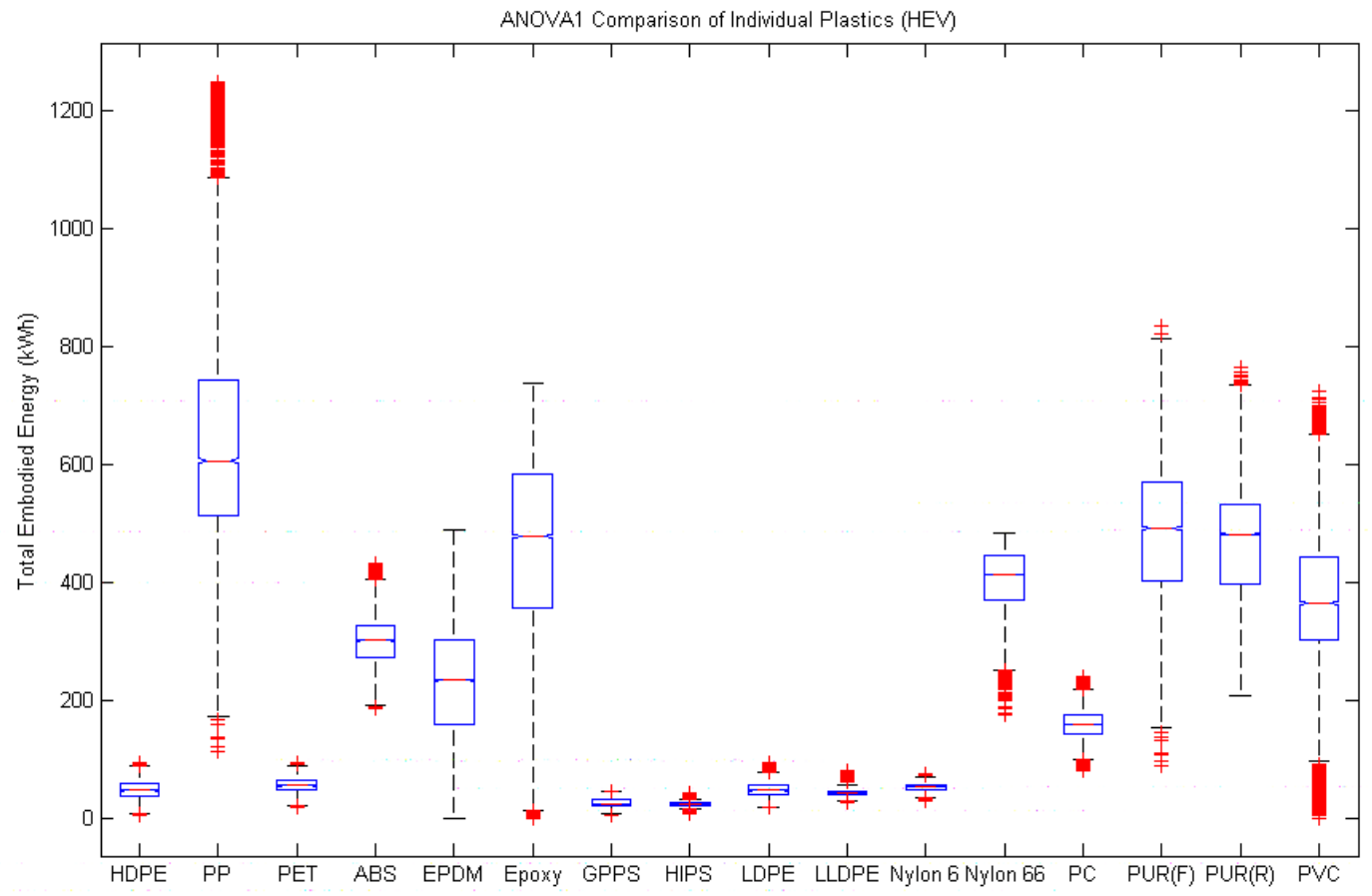

Figure 4.13 Box plots of an analysis of variance results for a hybrid electric vehicle (plastics only). 


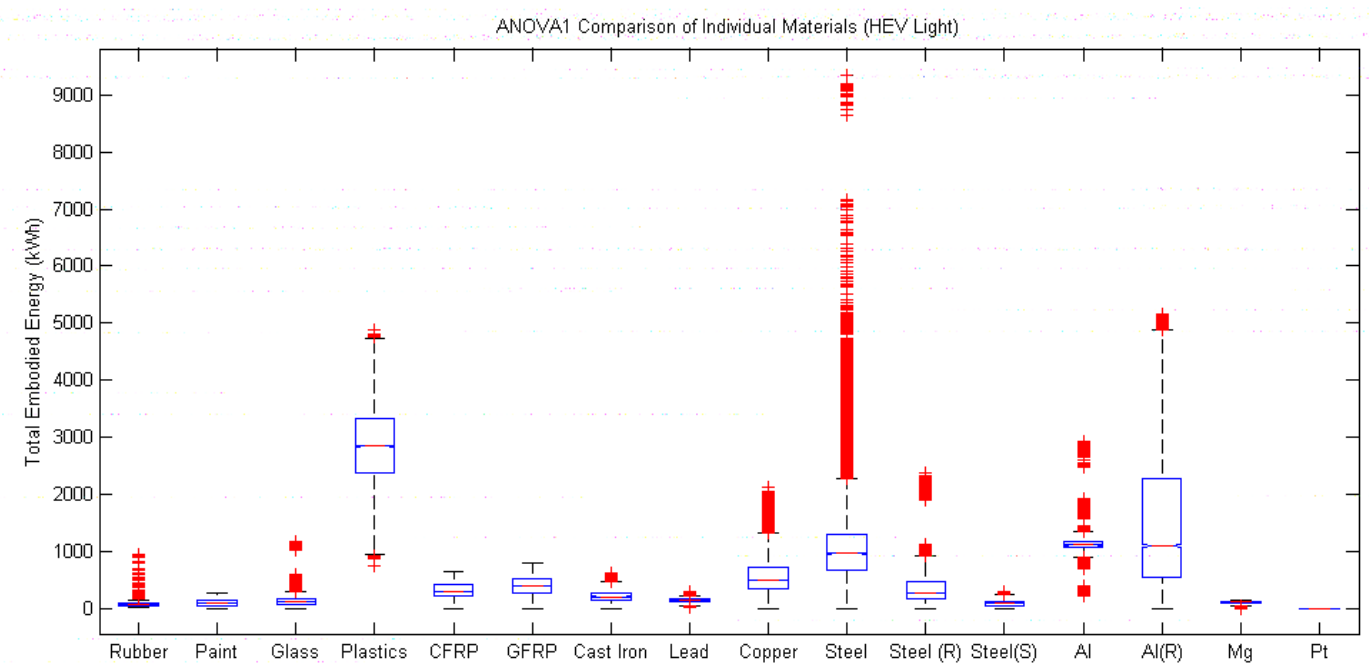

Figure 4.14 Box plots of analysis of variance results for a lightweight hybrid electric vehicle.

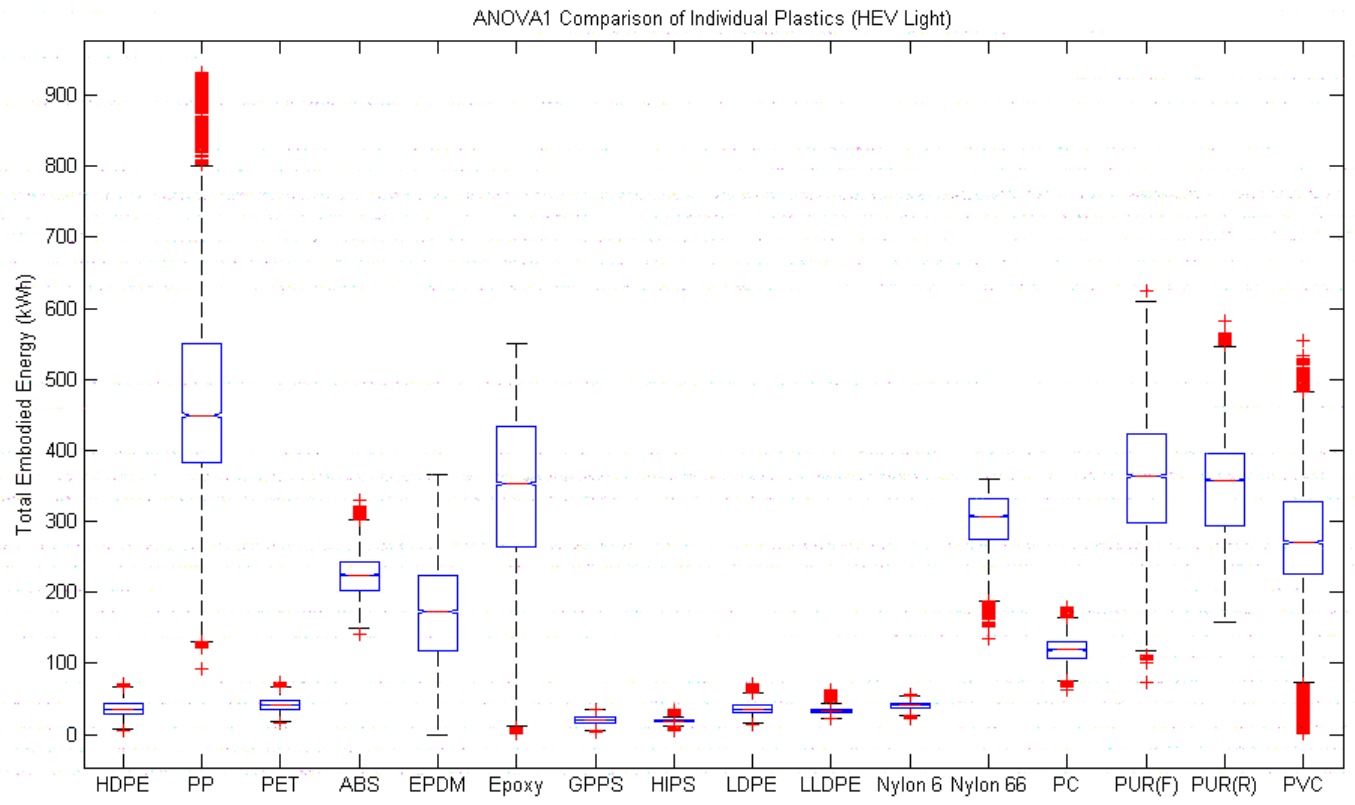

Figure 4.15 Box plots of analysis of variance results for a lightweight hybrid electric vehicle (plastics only). 


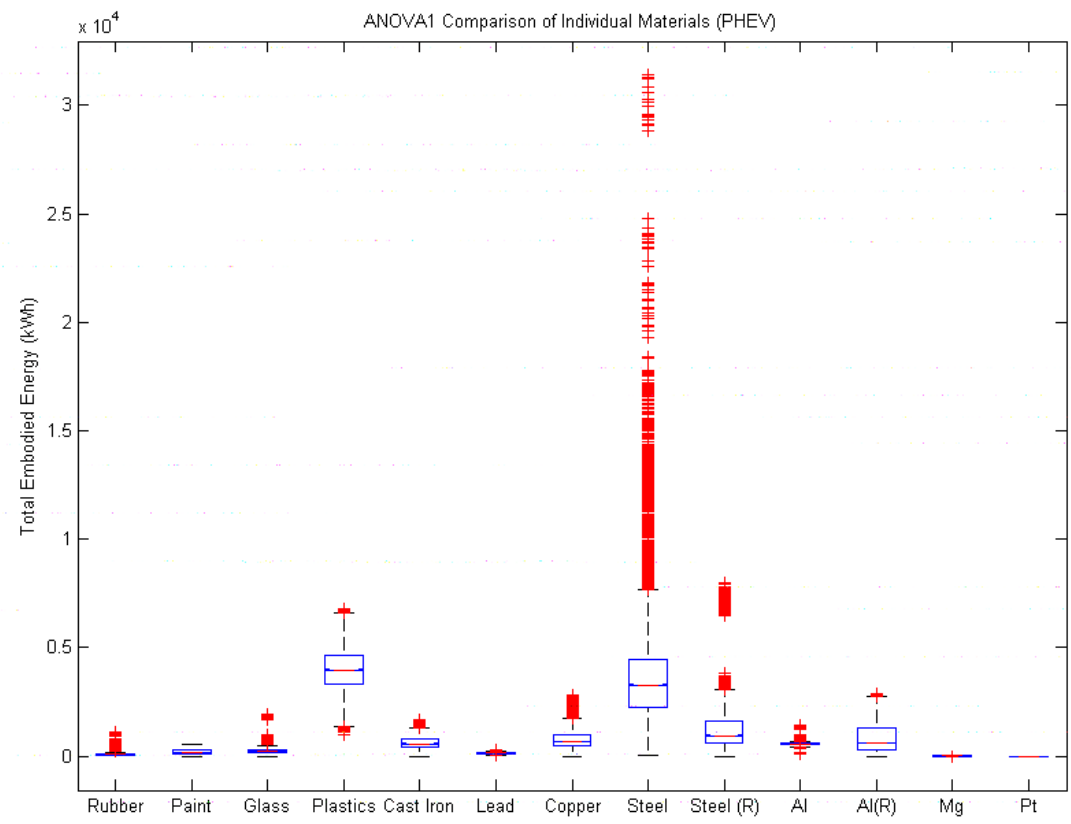

Figure 4.16 Box plots of an analysis of variance results for a plug-in hybrid electric vehicle.

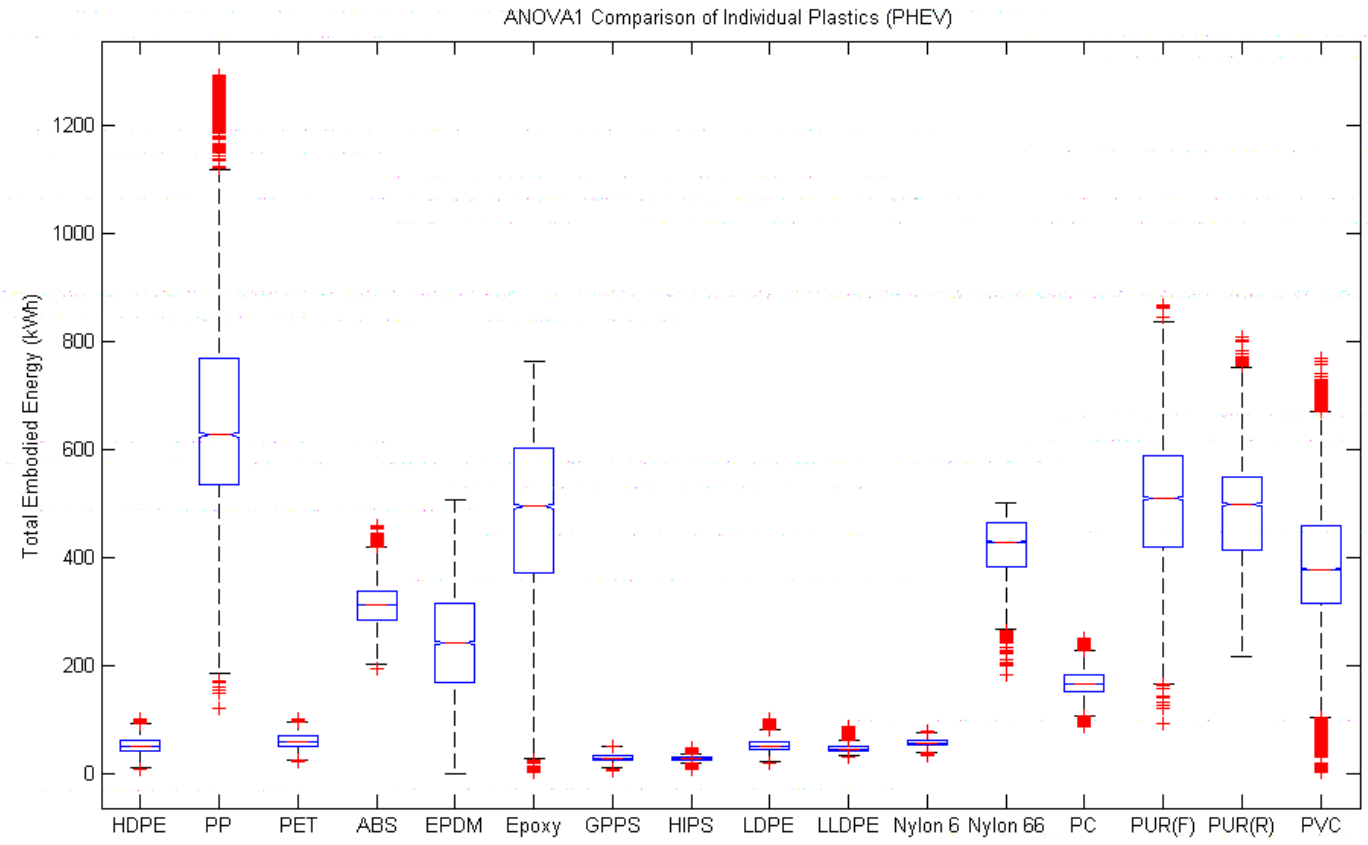

Figure 4.17 Box plots of an analysis of variance results for a plug-in hybrid electric vehicle (plastics only). 


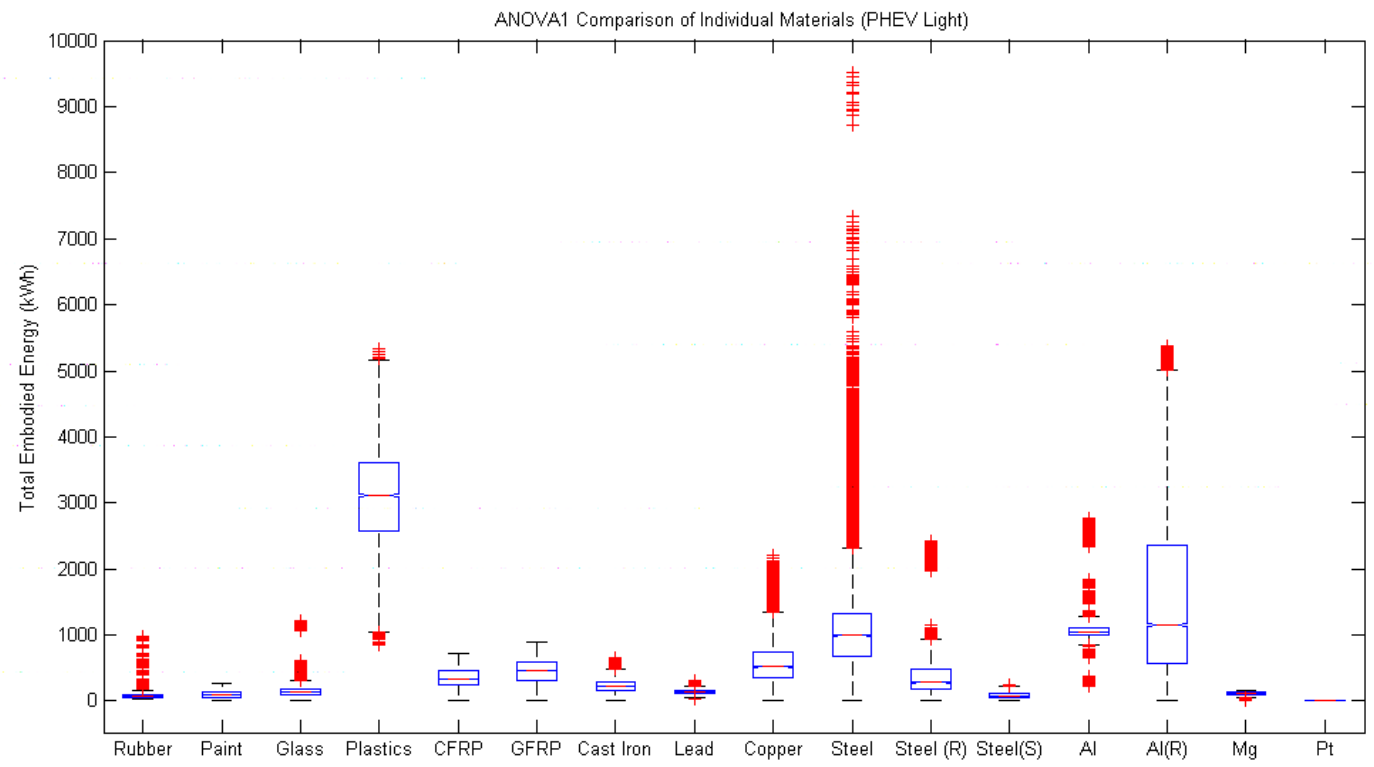

Figure 4.18 Box plots of an analysis of variance results for a lightweight plug-in hybrid electric vehicle.

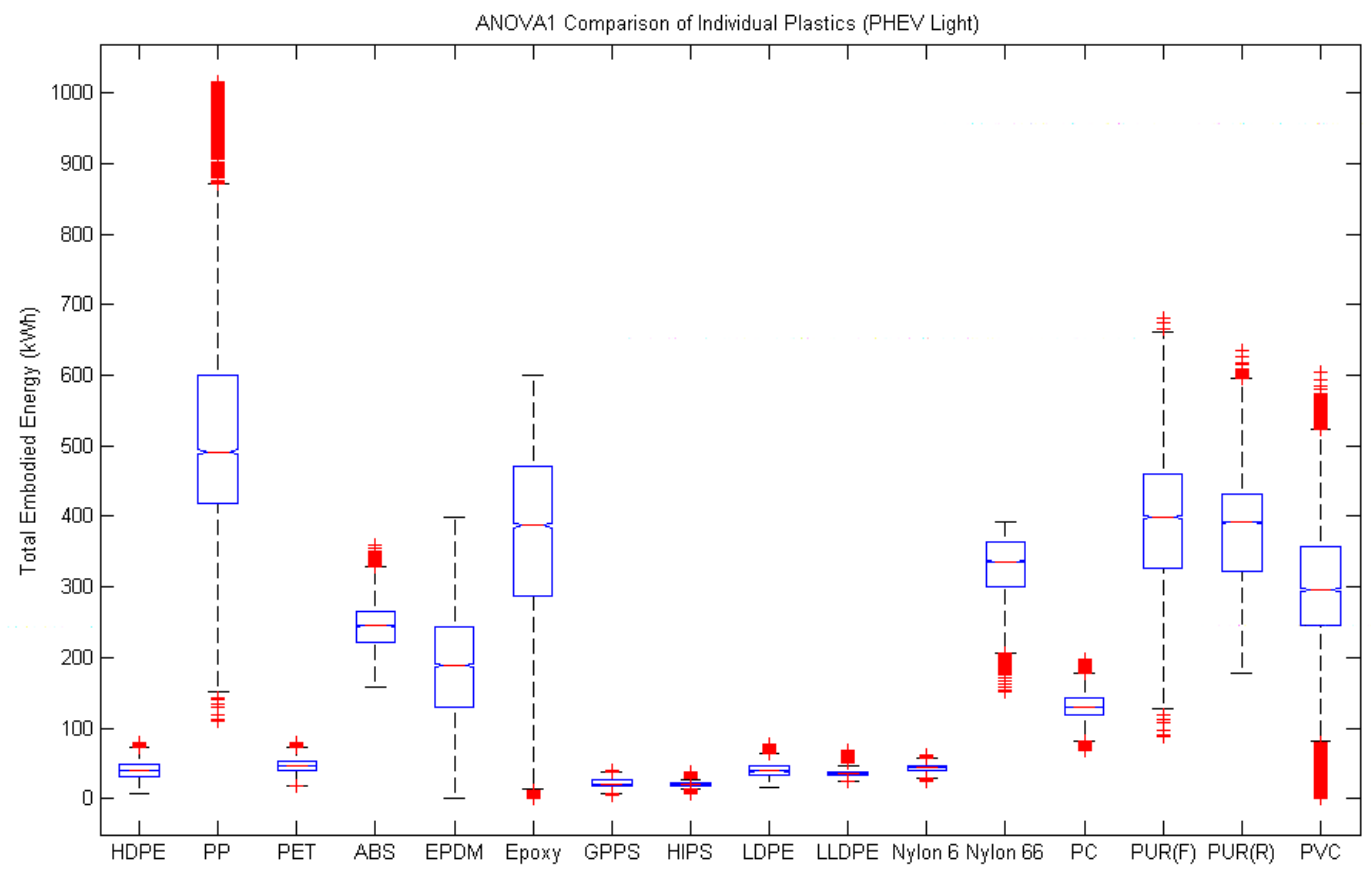

Figure 4.19 Box plots of an analysis of variance results for a lightweight plug-in hybrid electric vehicle (plastics only). 


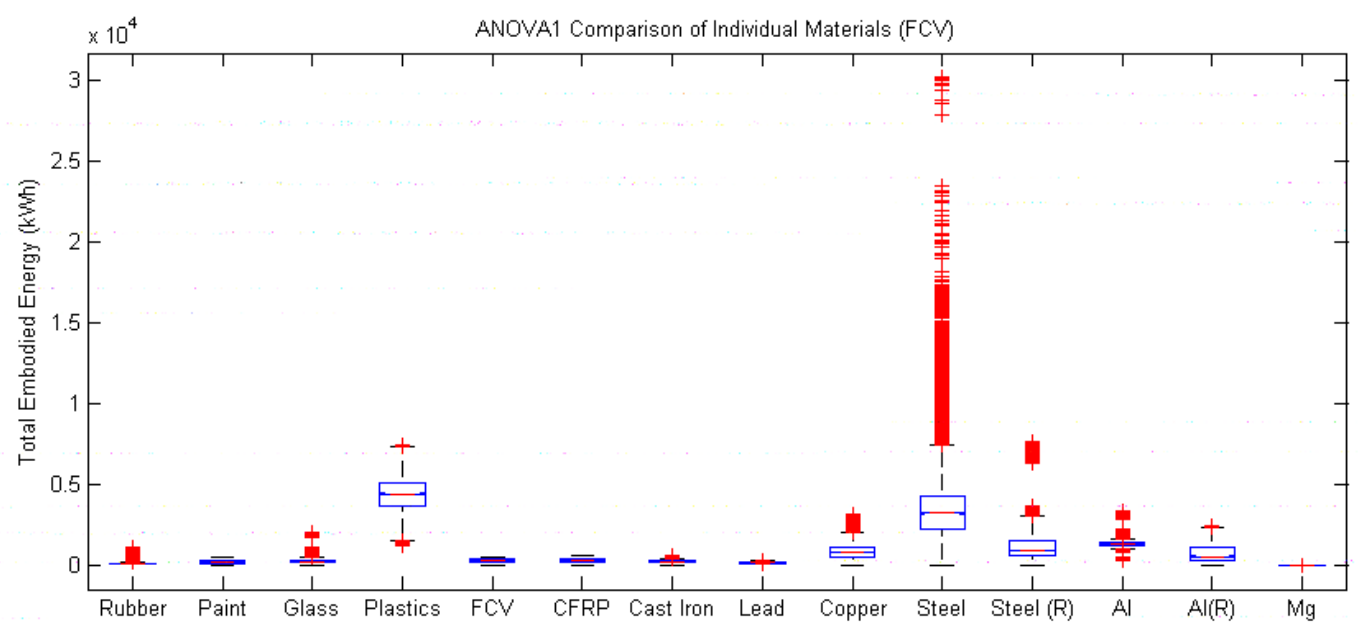

Figure 4.20 Box plots of an analysis of variance results for a fuel cell vehicle.

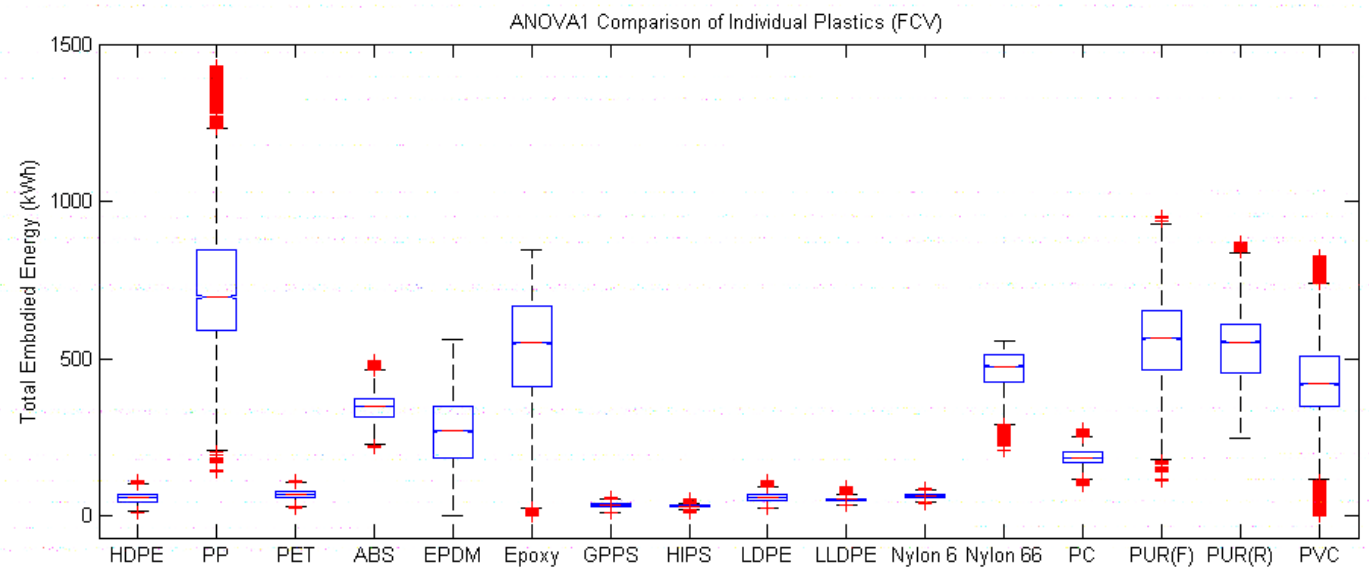

Figure 4.21 Box plots of an analysis of variance results for a fuel cell vehicle (plastics only).

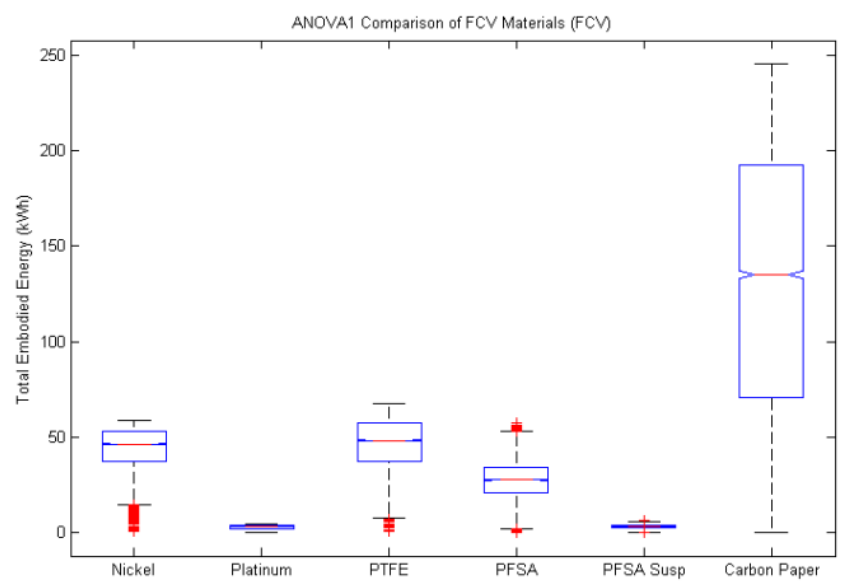

Figure 4.22 Box plots of an analysis of variance results for a fuel cell vehicle (fuel cell materials). 


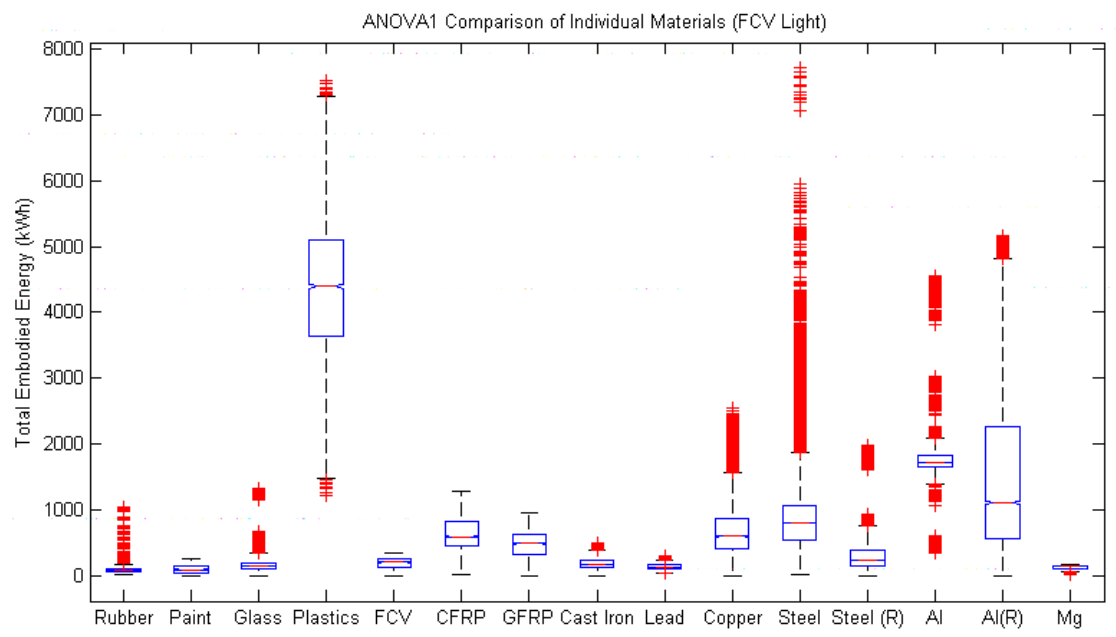

Figure 4.23 Box plots of an analysis of variance results for a lightweight fuel cell vehicle.

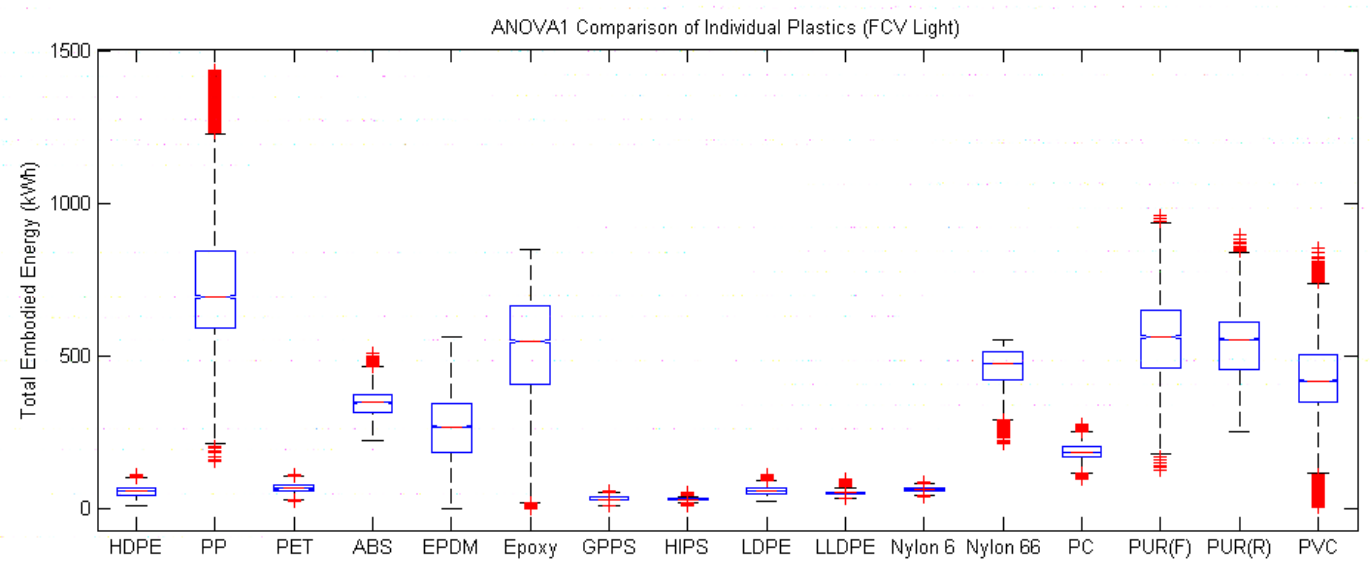

Figure 4.24 Box plots of an analysis of variance results for a lightweight fuel cell vehicle (plastics only).

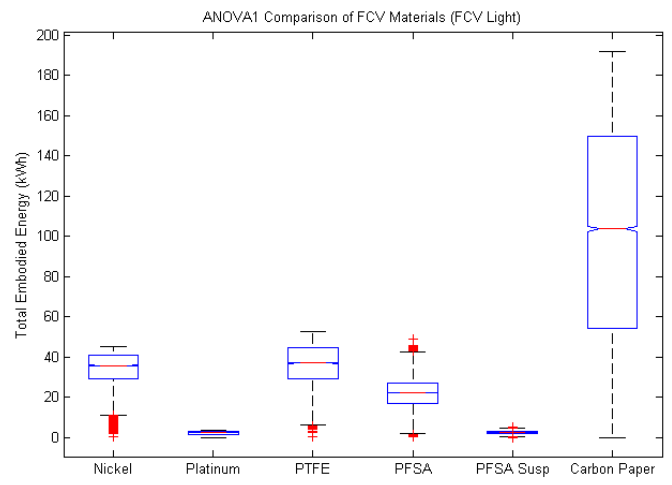

Figure 4.25 Box plots of an analysis of variance results for a fuel cell vehicle (fuel cell materials). 


\subsubsection{Regression analysis}

As mentioned earlier, the reason for utilizing a regression analysis is to determine whether the variance found in the Monte Carlo simulations can be attributed to temporal trends in energy intensity, or their absence. That is, what may appear as variance in a Monte Carlo simulation can merely reflect the condition of the data, namely a probability distribution which arises from a scattered data. Hence, regression analysis confirms or refutes whether this uncertainty is owing to temporality alone. The regression curve used here is of the first order format. Thus, a negative coefficient $p_{1}$ value indicates a downward slope that sees overall energy intensity $(\mathrm{kWh} / \mathrm{kg})$ reduced over time, while a positive coefficient sees energy intensity increasing over time. Higher order polynomials (second, third, etc.) are not presented here as there were no corresponding gains in terms of overall robustness. As mentioned earlier, a low root mean squared error $(R M S E)$ is an indication of a good fit. Materials which also show a high coefficient of determination value R-square $\left(R^{2}\right)$, a value close to 1.00 or $100 \%$, are said to have better approximations of the regression line to the data. Robust (LAR) analysis can construct a more trustworthy trend analysis; however, it must be said, this comes with the corollary assumption that outliers are non-representative. Table 4.12 displays the results of the regression analysis, both for the LS and LAR methods. Here, $\mathrm{N}$ represents the number of data points in the set and the " $+/-"$ indicates the $95^{\text {th }}$ percentile confidence interval bounds. It can be seen overall that the LAR analysis leads to much better fitting, as evidenced by the improvement of the R-square values, which are brought closer to 1.00 or $100 \%$. This can also be seen in the overall reduction in the $95 \%$ confidence intervals and the RMSE. However, it is not always the case that all of these act in concert. For example, EPDM sees better 95\% confidence intervals and R-square values while showing no change in RMSE values.

With the exception of PP and recycled steel, the LAR analysis found that most of the materials have a negative slope ( $p_{1}$ coefficient) indicating a decrease over time in energy intensity. Given the small size of $95^{\text {th }}$ percentile confidence bounds, R-square values close to 1.00 and low RMSE values, there is a considerable amount of assurance that the variance seen in some of the materials is owing to a historical trend. This seems true for rubber, paint, glass, PET, PP, lead, copper, steel, recycled steel, aluminum, and recycled aluminum. Conversely, the other materials 
in Table 4.12 show less evidence of historical trends. For example, materials with a negative Rsquare show no evidence of any historical trend. This includes materials specific to the fuel cell vehicle (PTFE, PFSA, etc.), magnesium, nickel, ABS, EPDM, Epoxy, GPPS, HIPS, HDPE, LDPE, Nylon6, Nylon66, PC, PUR and GFRP (see Table 4.1 for acronyms). Hence, it may be assumed here that, in these circumstances, there is uncertainty as defined in the Introduction chapter, specifically with respect to missing data. Based on the LAR and LS regression techniques, it may now be possible to calculate the change in each of the materials in energy intensity $(\mathrm{kWh} / \mathrm{kg})$ by percent. Accordingly, the number of years to see a $1 \%$ change in energy intensity values are listed by individual materials in Table 4.13. Hence, for the materials listed above which showed an actual historical trend (steel, aluminum, rubber, glass, etc.), 1\% changes in energy intensity occur roughly every two to five years. The choice of the $1 \%$ level of change was based on the standard significance level, or p-value. The derivation of these values did not depend on which regression technique was used because the time span in both cases of the LAR and LS was the same. Hence, the utilization of more robust techniques of fitting merely serves to bring about greater confidence that temporal variation is being accounted for. However, this does not mean that the other values in Table 4.13 (ABS, EPDM, etc.) should be ignored outright, but that some degree of caution should be exercised until further data is made available. Furthermore, this is not to suggest that the amount of variance in these materials should not be a matter of concern.

\subsubsection{Skewness and kurtosis}

As with the previous study, the data on individual materials was log transformed in order to test whether their distributions were non-normal. Tables 4.10 and 4.11 show the values for kurtosis and skewness, respectively. The row labelled "non-log" displays the kurtosis and skewness of the distributions prior to being $\log$ transformed while the " $\log _{10}$ " row displays the kurtosis and skewness after $\log$ transformation. It can be seen that in the majority of the cases the data

distributions are not non-normal. That is, their kurtosis values do not get closer to the ideal value of three and the skewness measure does not tend towards zero. The exceptions are with ABS, LDPE, and PC; however, their improvement is very marginal, especially in terms of kurtosis. The only case in which log transformation of the distributions seems to have benefited was for 
Table 4.10 Table of kurtosis values for each material.

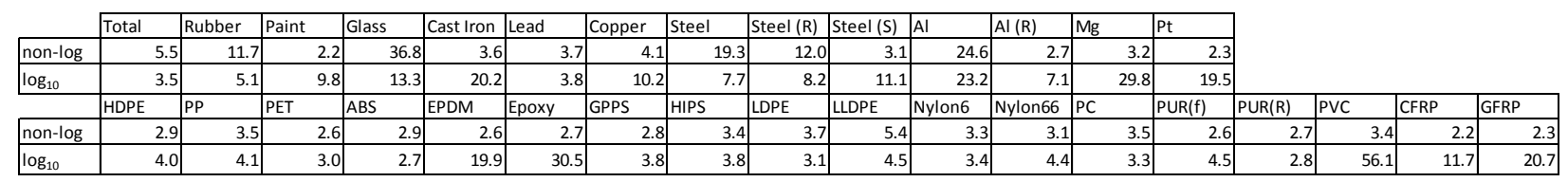

Table 4.11 Table of skewness values for each material.

\begin{tabular}{|c|c|c|c|c|c|c|c|c|c|c|c|c|c|c|c|c|c|c|}
\hline & Total & Rubber & Paint & Glass & Cast Iron & Lead & Copper & Steel & Steel (R) & Steel (S) & Al & Al (R) & $\mathrm{Mg}$ & $\mathrm{Pt}$ & & & & \\
\hline Non-log & 1.35 & 2.99 & 0.37 & 5.00 & 0.57 & 0.82 & 1.23 & 3.30 & 2.92 & 0.66 & 3.86 & 0.88 & -0.75 & -0.59 & & & & \\
\hline \multirow[t]{2}{*}{$\log _{10}$} & 0.01 & 1.68 & -1.88 & -1.56 & & -0.19 & -1.04 & -0.31 & -0.69 & -1.83 & -1.06 & & -2.78 & -3.03 & & & & \\
\hline & HDPE & PP & PET & ABS & EPDM & Epoxy & GPPS & HIPS & LDPE & LLDPE & Nylon6 & Nylon66 & PC & PUR(f) & PUR(R) & PVC & CFRP & GFRP \\
\hline Non-log & 0.26 & 0.59 & 0.23 & 0.16 & 0.02 & -0.60 & 0.50 & 0.52 & 0.80 & 1.79 & 0.04 & -0.68 & 0.66 & -0.14 & 0.07 & 0.20 & 0.24 & -0.13 \\
\hline $\log _{10}$ & -0.68 & -0.44 & -0.37 & -0.23 & -2.56 & -3.57 & -0.30 & -0.61 & 0.12 & 1.39 & -0.37 & -1.06 & 0.14 & -0.90 & -0.45 & -4.46 & -0.80 & -2.02 \\
\hline
\end{tabular}

Table 4.12 Regression analysis for energy intensities of materials of manufacture using least absolute regression and least squares regression methods.

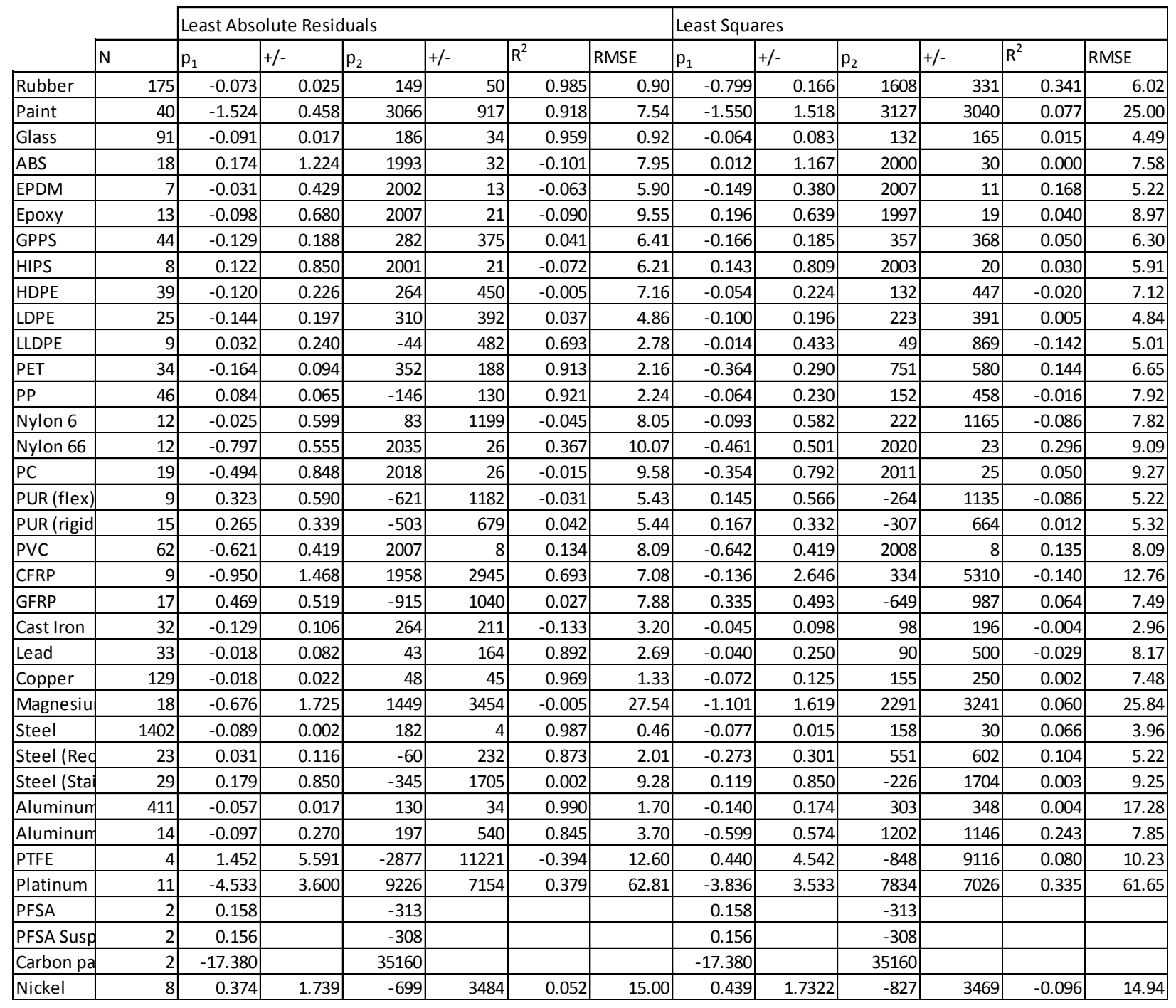


the total energy of the vehicle (the column labelled "Total") which saw its kurtosis and skewness reduced from 5.5 to 3.5 and 2.40 to 0.15 , respectively. Hence, this case would benefit from $\log$ transformation should it be desired to calculate the geometric mean of the distribution; that is, the mean calculated once the data has been transformed back to normal. For example, the total arithmetic mean energy for a regular ICEV in Table 4.6 was $11,719 \mathrm{kWh}$. Once the data was $\log$ transformed $\left(\log _{10}\right)$ and a Monte Carlo simulation run, the log transformed data was transformed back (using $10^{\mathrm{x}}$ or the power of ten), yielding a geometric mean of $10,356 \mathrm{kWh}$. Consequently, in cases such as these, new coefficients of variance can be calculated for more accurate results and, thus, yield better comparative assertions than were originally made in Table 4.6. Therefore, Table 4.14 shows the corrected means after the log transformation of the total energy per vehicle. Because of the positive skew of these distributions, there is the tendency to see a decrease in the mean values and their corresponding $C V s$. However, it will be noted that this has significantly more importance, for example, with lightweight electric vehicles than it does for lightweight fuel cell vehicles. Comparing $C V s$ sees $28.6 \%$ increase for the former, while only a $3.8 \%$ increase with the later.

\subsubsection{Caveat regarding data availability}

Very little data exists on key fuel cell (FCV) materials such as PTFE (known commercially as Teflon®), PFSA (a.k.a., Naphion®), PFSA suspension and carbon paper (the gas diffusion layer). The particular reasons for this lack of data are complicated. PFSA proton membranes require the use of platinum as a catalyst and, therefore, this increases the cost of production. Similarly, the poor conductivity performance characteristics of PFSA have been found to be deficient at temperatures above 80 degrees Celsius. Consequently, a great deal of research currently focuses on attempting to find substitute proton exchange membrane materials and catalysts (Peighambardoust et al., 2010; Bose et al., 2011; Chandan et al., 2013). Thus, there is the possibility that these materials (PTFE, PFSA, etc.) may never meet performance and cost targets as set by the U.S. Department of Energy (2013). That said, the overall energy impact in the Monte Carlo simulation for these materials (including platinum) is approximately $1.81 \%$ $(255 \mathrm{kWh})$ and this is consequently deemed minor in terms of their effect on the findings of this study. 
Table 4.13 Number of years for a one percent change in energy intensity $(\mathrm{kWh} / \mathrm{kg})$.

\begin{tabular}{|l|r|l|r|}
\hline ABS & 4.76 & Cast Iron & 2.56 \\
\hline EPDM & 7.69 & Lead & 2.56 \\
\hline Epoxy & 3.23 & Copper & 2.27 \\
\hline GPPS & 2.94 & Magnesium & 3.45 \\
\hline HIPS & 11.11 & Steel & 1.96 \\
\hline HDPE & 2.56 & Steel (R) & 3.57 \\
\hline LDPE & 2.94 & Steel (S) & 3.03 \\
\hline LLDPE & 3.13 & Aluminum & 2.27 \\
\hline PET & 3.13 & Aluminum (R) & 3.85 \\
\hline PP & 2.63 & Rubber & 2.27 \\
\hline Nylon 6 & 3.12 & Paint & 5.26 \\
\hline Nylon 66 & 3.03 & Glass & 2.27 \\
\hline PC & 3.13 & CFRP & 4.35 \\
\hline PUR (flex) & 4.76 & GFRP & 3.45 \\
\hline PUR (rigid) & 3.13 & PTFE & 8.33 \\
\hline PVC & 3.03 & Platinum & 3.13 \\
\hline \multirow{2}{*yyy}{} & & PFSA & 8.33 \\
\cline { 2 - 4 } & & PFSA Suspension & 8.33 \\
\cline { 2 - 4 } & & Carbon paper & 8.33 \\
\cline { 2 - 4 } & & Nickel & 4.35 \\
\cline { 2 - 4 } & & &
\end{tabular}

Table 4.14 A comparison of total energy per vehicle before and after log transformation (kWh).

\begin{tabular}{|c|c|c|c|c|c|c|c|}
\hline & & \multicolumn{3}{|c|}{ Pre Log Transformed } & \multicolumn{3}{|c|}{ Log Transformed } \\
\hline & & Mean & Std Dev & CV & Mean & Std Dev & $\mathrm{CV}$ \\
\hline \multirow[t]{2}{*}{ ICEV } & Regular & 11,719 & 6,420 & 0.548 & 10,356 & 6,420 & 0.620 \\
\hline & Lightweight & 8,938 & 4,326 & 0.484 & 8,022 & 4,326 & 0.539 \\
\hline \multirow[t]{2}{*}{ EV } & Regular & 16,788 & 9,496 & 0.566 & 14,740 & 9,496 & 0.644 \\
\hline & Lightweight & 12,970 & 5,998 & 0.462 & 8,022 & 5,998 & 0.748 \\
\hline \multirow[t]{2}{*}{$\mathrm{HEV}$} & Regular & 12,491 & 7,107 & 0.569 & 10,947 & 7,107 & 0.649 \\
\hline & Lightweight & 9,389 & 4,553 & 0.485 & 8,429 & 4,553 & 0.540 \\
\hline \multirow[t]{2}{*}{ PHEV } & Regular & 12,494 & 7,119 & 0.570 & 10,947 & 7,119 & 0.650 \\
\hline & Lightweight & 9,804 & 4,730 & 0.482 & 8,806 & 4,730 & 0.537 \\
\hline \multirow[t]{2}{*}{ FCV } & Regular & 14,029 & 7,459 & 0.532 & 12,500 & 7,459 & 0.597 \\
\hline & Lightweight & 11,978 & 5,102 & 0.426 & 10,995 & 5,102 & 0.464 \\
\hline
\end{tabular}




\subsection{Conclusions}

This study expands upon previous work by more than doubling the number of materials of manufacture from 17 to 36 and by further developing the normative platform for examining the issue of temporality due to age of data. Again, Monte Carlo simulations were used in order to characterize the variance in each of the 36 individual materials of manufacture. Regression analysis was also used alongside the simulations, both in the form of least angle regression and least absolute regression. The purpose of the regression analysis was to serve as a confirmation whether the variance seen in the Monte Carlo simulations was a temporal correlation or purely variance. Both regression methods did yield temporal relations for a number of materials such as aluminum, rubber and glass. However, given that the least absolute regression method found much better fits to the data, this method is preferred in order to ascertain temporal relations with definitive confidence. The regression analysis also served to measure the number of years over which percentage changes occurred. Not surprising, the least angle regression and the least absolute regression methods did not yield any differences in terms of percentage change since they consider changes over the same time frame. Hence, neither the slope of the regression line nor the robustness of fit come into consideration here. That said, the percentage changes could be expressed as a significance level (i.e., one percent or five percent). Nevertheless, many materials such as steel, aluminum and copper showed a one percent shift in values between two and three years. This is purposeful in establishing future norms of practice regarding updating data life cycle assessment studies. Again, the issue of non-normality was revisited by log transforming the energy distributions, re-running the Monte Carlo simulations and then retesting for changes in kurtosis and skewness. For the individual materials of manufacture, only marginal improvements in kurtosis and skewness were seen form ABS, LDPE and PC. Thus, there was little utility in log transforming for these materials. However, at the functional unit level (the whole vehicle), there was significant reductions in kurtosis and skewness. Hence, the log transformed energy distributions can be converted back in order to derive a new geometric mean (as opposed to the pre-transformed arithmetic mean). This would be useful should more accurate comparative assertions be desirable by comparison of newly derived coefficients of variation. 


\section{CHAPTER 5-CONCLUSIONS}

\subsection{General Conclusions}

This study identified a research gap which has implications for the robustness of life cycle assessments. This gap was contextualized in the overall drive towards standardization of life cycle assessments as envisaged by the ISO14040 series of standards. The specific target research area identified a lack of accounting for temporality and spatiality due to age of data. Consequently, an examination of the energy intensity of 36 individual materials of manufacture across several decades (roughly 1960 to 2012) was conducted to see whether there was a temporal relation which could impact the outcomes of life cycle assessment studies. Similarly, the energy intensity of materials of manufacture originating from three different geographic areas across the same time spans were compared to see whether there were differences attributable to spatial origin. This required the collection of data through a vast literature search which lasted over a year. The model chosen to test whether these phenomena were real was the Greenhouse gases, Regulatory Emissions and Energy in Transportation 2 (GREET2) by the Argonne National Laboratory. Since GREET2 model the entire life cycle of automobiles, this model was deemed sufficiently complex for testing the phenomena of temporality and spatiality due to age of data. It also had a number of potential configurations which made possible comparative assertions: lightweight versus regular material vehicles; sedans, pick-up trucks and sport utility vehicles; and different potential powertrain scenarios: internal combustion, hybrids, plug-in hybrids, electrical, and fuel cell. Standard methods were used and consisted of Monte Carlo simulations programmed in MATLAB, as well as built-in statistical toolkits in MATLAB for the analysis of variance and regression analysis.

Monte Carlo simulations allowed for the construction of a probabilistic distribution of the potential outcomes of energy intensity of individual materials of manufacture used in the GREET2 model across their full time frame. Once the simulations were programmed and executed, statistical data was collected regarding their resultant distributions, including standard deviation, standard error, arithmetic mean, and coefficient of variance. An analysis of variance was also conducted to render each of the variations in terms of individual materials of 
manufacture within the prescribed physical properties of the GREET2 model. This allowed for the ability to visualize how mass became involved in the overall amount of variance. The filtering of data through geographic regions allowed for spatial comparisons once the Monte Carlo simulations were run. Using another version of analysis of variance (F tests) allowed for the measurement of the degree of differences between groups (geographic areas). Where differences were significant (critical), effects due to spatiality were seen. Furthermore, regression analysis was conducted on the raw data used for the temporality studies in order to confirm whether the variance seen after the Monte Carlo simulations were run was due to an actual temporal phenomena, or just pure variance. Both a least squares regression and a least absolute regression analysis were used to check for temporal phenomena and test the wellness of fit (robustness). Additionally, both regression methods were used to find the amount of percentage change which occurred in the energy intensity of individual materials of manufacture. Lastly, because of the concern for non-normality in the energy distributions, further Monte Carlo simulations were conducted using log transformed data to test for non-normality. The key metrics used in this testing for non-normality were skewness and kurtosis.

\subsubsection{Contributions}

This study showed that the effects of temporality and spatiality owing to the age of data are not insignificant or ignorable. This was demonstrated through the presence of large amounts of variance both on the basis of the functional unit as well as on an individual material basis. On a functional unit basis, a significant variance was shown in the total amount of energy over the full time scale. However, this study did show that — on the basis of the functional unit — these effects are dependent on the particular mixture (weight) of materials utilized, spatial location, as well as the time frame which is being examined. Not surprising, heavier vehicles saw larger mean total energy values and larger variation in their distributions. This indicates the importance of weight in characterizing the effects of aged data for a functional unit. Thus, lightweight vehicles which use substitute materials such as aluminum to reduce overall vehicle weight saw much smaller mean total energy. However, examining the effects of individual materials of manufacture reveals a more complicated image. Using analysis of variation (ANOVA), the materials which saw the highest evidence of variation in the full time frame were steel (primary and recycled), 
recycled aluminum, cast iron, copper, HDPE, PP and PET. Furthermore, it was shown that spatial considerations are intertwined to varying degrees with temporal variation for some materials with the exception of copper and aluminum. Consequently, temporal and spatial changes in the energy impacts of a functional unit should give more consideration to the energy intensity $(\mathrm{kWh} / \mathrm{kg})$ through more mixed methods (see below).

This research is important in that it established that the input stages of a process captured by LCA, more specifically related to LCI, are just as important as the output stages in terms of the effects of the age of data and data quality. The findings point to what has been a subject hitherto ignored in previous studies on the effects of age of data temporality and spatiality. The implications of this research are not inconsequential. While it is known that uncertainty characterizes the LCA process, these findings renew the need for further scrutiny of data such that data collection should be routinely updated in order to make properly informed functional unit comparisons, which — as was mentioned in the introduction - is one of the central purposes of LCA. Depending on the type of material, the precise amount of time these updates should occur generally ranges between two and five years. Furthermore, these updates should be investigated for the entire spectrum of materials (e.g., cement, timber, ceramics) as this does not appear to be isolated to a single material or class of materials (e.g., plastics, metals, etc.). Although no LCA study is lost to time, this dissertation shows that LCA studies remain rooted in a specific time. But this time can be highly confusing when dealing with increased functional unit complexity which necessitates drawing upon many different sources of data. Indeed, some LCA studies reviewed during research for this study revealed that some of their source data for energy intensity was dated, in some cases by more than a decade. Thus, if data utilized in an LCA study stretches across a ten year interval, it is difficult to ascertain what reality the results reflect - the reality of year 1, year 5, or year 10? Or some range in between? In turn, this can have consequences in terms of whether LCA will have any longevity as a viable assessment tool. Also, data promulgation and gathering is a very time-consuming process in this area of study; however, this is a necessary trade-off and should be a priority to bring about more robust results.

In addition, the regression analysis indicated which of the materials are subject to uncertainty owing to missing data. Regrettably, this is the case for 19 of the 35 materials in this study, 
although their overall contribution in the Monte Carlo simulations for the total energy of a vehicle (as a functional unit) is small. Consequently, work should be done to collect more in situ data from manufacturing sources. The inconclusive regression analysis for some materials in Table 4.12 serves as evidence for much more aggressive data collection in this area. This is largely due to the large amounts of variance seen in these materials in spite of the inability to make a definitive temporal association. Yet, regardless of the condition of the data, the direct temporal correlations found for materials such as steel, aluminum, etc., make for a troubling situation concerning the usefulness and representativeness of some LCA results. At a minimum, it should be obvious that metadata precisely on the age of data does not address this problem but only serves to denote the timeframe in which an LCA remains relevant. This means that not only do data inputs (LCI) need to be updated regularly but, in turn, so must LCAs in order to make them relevant.

One of the research questions this study sought to address was whether there is utility in analyzing temporality and spatiality due to age of data at both the functional unit level and at the individual materials of manufacture level. Analysis at the level of the functional unit allows for comparative assertions with other similar functional units. This is one of the explicit purposes as envisioned by ISO14040. In the case of this study, the functional unit is an automobile. The constituent materials of each automobile — as presented by weight composition in the beginning of chapters 3 and $4-$ vary not inconsiderably. And, this seems amply true in the case of this study. However, as was seen, more mass of materials merely means greater total energy and greater variance of a scalable nature. As was presented, using the full time scale showed that the coefficients of variance were scalable. Hence, differences between functional units mean that there are scalar fluctuations within the functional unit. A more interesting phenomenon occurs when observing the individual materials of manufacture since some materials have vastly higher contributions to the overall variance than others. For example, it was thought during the initial stages of this research that, given the very high energy intensity of glass and carbon fibre reinforced plastics, would make the lightweight vehicles consume much more energy than the regular vehicles. In fact, this was not the case. This means that much more attention must be given to the internal dynamics of a functional unit than the functional unit as a whole. In order to bring this about requires a set of methods including Monte Carlo simulations, regression 
analysis, analysis of variation, and tests for non-normality. The precise usage of these methods are given below.

\subsubsection{Implications}

This study has a number of implications for what in life cycle assessments is referred to as quality of data issues. However, this implication is highly specific. The actual framework of a life cycle assessment is not being questioned here, nor are the findings which arise from the use of data rooted in a specific time and place. Rather, what is implicated here is the degree to which, for example, an LCA conducted in 1990 (using data from the same year) is specific to 1990. As a competitor developing a similar product with similar functionality (say, in 1995), there would be considerable interest answering this question. As such, this subject is a question of confidence, robustness and representativeness. Because these questions have not previously been answered in the domain that is the subject of this study, guidelines should be developed in order to make answering these questions easier. Subsequently, this study looked into the potential methods for examining temporal and spatial effects due to aged data in the life cycle assessment. A number of recommendations resulted from this:

1) Examining the effects of temporality and spatiality due to aged data should primarily be on the basis of individual materials of manufacture and not the functional unit.

2) The variance arising from Monte Carlo simulations should be taken as the primary measurement of the effects of temporality due to age of data. As per 1), these effects should be measured for each individual material and may be visually represented by box plots.

3) The variance arising from the Monte Carlo simulations should be measured across the entire time span of available data as smaller time intervals can result in erroneous representations of variance.

4) As a result of 3), comparison of coefficients of variance from different time intervals should be avoided, as well as analysis using F values (ANOVA) under the same conditions. 
5) The effects of spatiality can be isolated by measuring differences through $F$ values between separate geographic regions in the same time frame and the same weight for individual materials.

6) In spite of apparent skewness in data, log transforming the data has little effect in bringing about the potential for more clarity. Nevertheless, as a method, $\log$ transformation can be used to test the potential assumption of linear versus log-normal data sets by testing for skewness or kurtosis (both are embedded MATLAB functions). This would also be necessary should it be desirable to derive the geometric mean (as opposed to the arithmetic mean shown in the majority of the tables in this study).

Some further recommendations with regard to methods also arose from this study. Chief among these is the inclusion of a robust regression method (least absolute residuals) in order to confirm that the variance seen in the Monte Carlo simulations is due to actual temporal variation. However, neither the least absolute residuals nor the least squared method provided more accurate measurements of the changes in energy intensity ( $\mathrm{kWh} / \mathrm{kg}$ over time). In either case, they proved equally useful since their regression lines spanned the same time period. Another method tested whether the data was non-normal by measuring kurtosis and skewness before and after $\log$ transformation in base 10. In all cases this was found not to be necessary for individual materials. However, in the case of the total vehicle energy consumption, there was some utility in log transforming the data, rerunning the Monte Carlo simulation, and then transforming the data back in order to derive a new geometric mean and, hence, a corrected coefficient of variation. Therefore, tests for kurtosis and skewness should be included as part of an overall mixed methods platform of analysis.

\subsection{Limitations of This Study}

According to Van den Berg (1999), with LCA there is no such thing as a feedback mechanism. Rather, the confidence in outcomes is based on the quality of the input data and the quality of the 
models used. As an issue, data quality analysis in LCA can be hampered by a number of factors (Ibid.):

1) Lack of metadata which hampers calculation of error propagation.

2) Differences in types of data input (e.g., weighting might have been employed).

3) The model in LCA cannot be tested. Comparing data to in situ situations is cumbersome. Temporal and spatial characterizations are lacking. The character of a stead state model does not allow for predictions to be made.

4) The number of inputs in an LCA is large (e.g., a medium LCA can consist of 500 processes with 200 items per process), thus making independent reliability issues accumulate.

5) Individual impact categories determine confidence in the results. Hence, one category can have a certain level of confidence which varies significantly with another category.

This study implicates two impacts as outlined by Van den Berg (1999), namely points 3 and 5. It is affected by the fact that the model is testing temporal and spatial characterizations utilizing in situ data. However, this operates under the assumption that the model is testable. This assumption arises from the fact that Monte Carlo simulations are applicable to testing for uncertainty and variance (see Chapter 3.2.1). Thus, although it was not the intention of this study to do so, it has made some normative assertions with regard to the testing of LCA models. Whether that can be generalized to other facets of LCA models is unknown and would require further investigation. Also, the validity of the input data has implications as to whether the findings can be generalized to other environments or circumstances. The work in this dissertation is limited to only one impact category within LCA: energy intensity. The effect of temporality due to age of data does not affect other general impact categories such as ozone depletion, abiotic resource use, biotic resource use, acidification, eutrophication, aquatic/terrestrial ecotoxicity, and human toxicity as outlined in Pelletier et al. (2007). This is due to two fairly clear issues. In the first case, resource use is an economic issue and, hence, is related to stocks and surpluses (amounts). Although the stock of a particular resource may change over time, its functionality is retained on a per unit basis, be that mass, volume, etc. In 
the second case, all the other impact categories are dose dependent and, thus, have no temporal aspect due to the age of data. As such, the toxicological potential of equivalent amounts of toxins does not alter over time.

Further limitations of this study include the absence of data which limited the analysis to three geographic areas (North America, Europe, and Oceania) to the exclusion of Asia, Africa, as well as South and Central America. Additionally, those spatial areas examined were on the continental basis and, thus, expand across vast distances. This is of concern since, for example, Boustead and Hancock $(1979,1981)$ indicated that there does exist variance of energy intensity of manufacture $(\mathrm{kWh} / \mathrm{kg})$ within countries - in their case, across the UK. Hence, more specificity can be brought to bear in this area. Alongside this issue is that the studies, technical reports, etc., utilized here were all exclusively in English and German. Thus, other potential sources of data were not examined, assuming they existed at all. Further to this is that, for the materials of manufacture, only primary processes were examined for energy intensity, to the exclusion of secondary processes. Thus, the energy totals derived on the basis of the functional unit (vehicle) do not purport to be the total energy up to the point of the user phase of the LCA. As a consequence, the tabulated totals are merely a fraction of the cradle-to-gate segment leading up to the user phase. Also, this study does not purport to explain the sources of variance, be it changes in technology, power grid composition, etc.

This study looked at temporality and spatiality due to age of data as individual subject matters. As such, this study did not cross-correlate the temporal and spatial findings. At some point, this may be desirable but is easily accomplishable by conducting regression analysis on geographically specific data. Thus, this is a minor limitation.

\subsection{Future Work}

Going forward, there are a number of challenges to overcome in studying this area further. One particular challenge is the issue of the indeterminacy of the origins of variation in the data. This is disquieting because the origins of this variation are not readily known or explainable. Either these origins are valid and represent actual in situ realities, or they are due to unfathomable 
conditions (e.g., error, which is a form of uncertainty). However, this may all be irrelevant because the only solution to the issue - in either case - is the promulgation of more data. This objective is obviously hampered by current circumstances in the market of knowledge. Indeed, it is public knowledge that, through the 1930s to the late 1960s, DuPont invented Nylon, Teflon® (PTFE) and Nafion ${ }^{\circledR}$ (PFSA). To date, DuPont does not provide any publicly accessible energy intensity data on the last two items in this list. However, in the case of the energy intensities of both Nylon 6 and Nylon 66, DuPont published on its own website Boustead's 2005 LCA studies (Boustead 2005a, 2005b) which were conducted on behalf of Plastics Europe, the Brussels-based institution. Naturally, Boustead's results relate to manufacturing in Europe and not North America. Why this situation with data persists is perplexing and whether it is indicative of this industry or is due to proprietary measures is unknown.

Also of concern is the issue of the regression analysis utilized here because this method did not take into account theoretical energy intensity minimums. This can have the effect of making the regression analysis appear as if it were not going to plateau at some imminent future period. For example, the minimum theoretical limit for virgin steel is given to be $4.67 \mathrm{kWh} / \mathrm{kg}$ (Worrell et al., 1993) which is close to the terminal values found in the regression analysis used here.

Conversely, the theoretical minimum for virgin aluminum is $6.34 \mathrm{kWh} / \mathrm{kg}$ (Grjotheim et al., 1995), a level which the regression analysis showed is a far distant goal. Thus, this must be kept in mind when viewing the results of this study, and further modelling should be done. That is, the regression lines should not be interpreted as a predictor of the trajectory of future events. Certainly, scenario analysis would be a much more sound line of inquiry into this subject, should it be done at some future time.

One area that must be examined is the issue of metadata or, as it is known in more colloquial terms, "the data on the data." Metadata on the age and place of data could be purposeful in outlining the practical usefulness of energy intensity source data. As part of the consistency check, ISO14040 requires life cycle assessments to make note of data sources, data accuracy, technology coverage, time-related coverage (how old is the technology being utilized), data age, and geographical coverage. Hence, given the already heavy burden of data in life cycle assessments, this would seem at first hand to make life cycle assessment even more cumbersome. 
At this time, it is not certain as to whether this would even be desirable, except beyond administrative purposes. As such, this area is highly speculative at this time but is further elaborated below.

During the data collection phase of this research, one particular observation which arose was the tendency of life cycle assessments to use data from different times. For example, a life cycle assessment conducted in 2001 on a fictional Product A utilized data for polyvinyl chloride (PVC) from 1990 and steel data from 1997. Consequently, the question here is in what space-time reality does the life cycle assessment for Product A exist. Certainly, it is not 2001, and it is not an interpolation between 1990 and 1997. This also poses a further quandary with quantitative implications if the functional unit in question is to be subject to comparative assertions with other functional units (say, Product B, also from 2001) using different materials with equally divergent data sources. From an assessment perspective, this poses a number of difficult questions. Should the data of the constituent materials of PVC and steel be adjusted to reflect the date of study in 2001 so that Product A and Product B can be compared? This would seem to be the case if Product A and Product B were made of completely different materials. But, this is only possible in the context of whether data on the constituent materials of manufacture for both Product A and Product B can be adjusted accordingly. For example, this would not be the case if Product $\mathrm{B}$ is made of a material with no time adjustable data (no temporal relation was found in the linear regression) and is subject to large amounts of variance. As such, this problem outlines a microcosm of a much bigger issue of the intersection between variance and uncertainty. Usually, these subjects are handled individually (see Huijbregts, 1998a), but an assessment of their synergistic affects should be made.

Upon reflection, it is evident that a considerable effort must be made with regard to the question of data quality and data representativeness for the furtherance of more valid results in LCA. As an assessment tool, LCA is naturally dependent on the quality of its data. Currently, LCA is problematic due to - among other things - a lack of consolidated methods and the haphazard usage of data. For example, Sutherland et al. (2008) use data from Boustead and Hancock (1979) as a source for the energy intensity of hydrochloric acid production. It should be apparent that the use of nearly thirty year old data is neither ideal nor desirable. Nevertheless, this should 
have been fairly clear long before this present study was done. However, moving forward from this study requires that LCA practitioners become more involved in the process of gathering data rather than relying on datasets which come with a set of largely unspoken assumptions regarding their source and representativeness. Much more "site-specificity" is required; that is, data should be coming both directly from the source and with far more regularity. This study also has further implications for a host of materials of manufacture not included here, along with the hundreds of unit processes used in LCAs. The continued use of low numbers of data points and assumed probability distributions (normal, lognormal, etc.) for modeling physical parameters within LCA (e.g., electricity usage, waste generated, water usage, etc.) is highly conducive of erroneous findings. These issues should be revisited with the aim of pursuing normative ends with respect to methods. The overwhelming focus with modeling uncertainty during the last twenty years is a tacit admission of the undesirable current situation with regard to data. Consequently, this should give way to moving away from "modeling" towards precision. As such, this means more purist empirical approaches should be pursued at the expense of abstractions. 


\section{APPENDIX A-PROBABILITY DISTRIBUTIONS}

PAGE

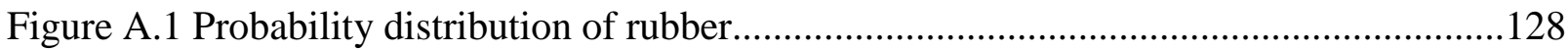

Figure A.2 Probability distribution of paint.................................................................128

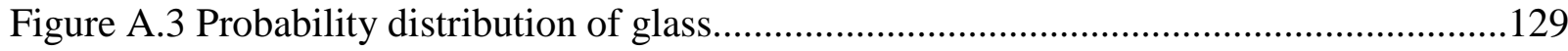

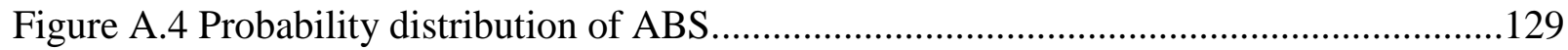

Figure A.5 Probability distribution of EPDM................................................................ 130

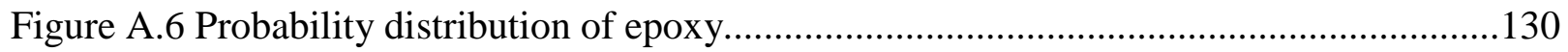

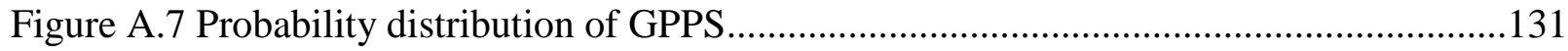

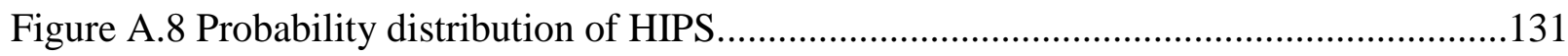

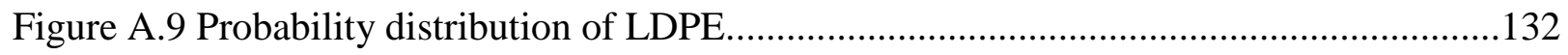

Figure A.10 Probability distribution of LLDPE.............................................................132

Figure A.11 Probability distribution of Nylon6..........................................................133

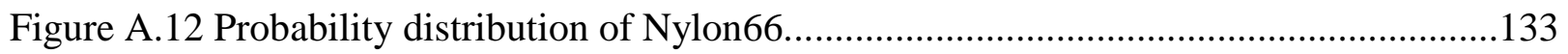

Figure A.13 Probability distribution of PC ................................................................134

Figure A.14 Probability distribution of PUR(F) .......................................................134

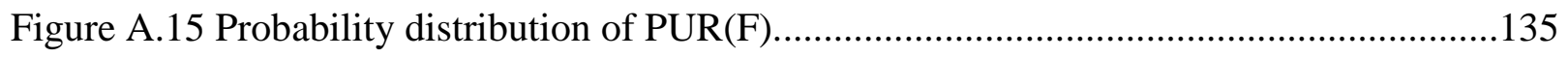

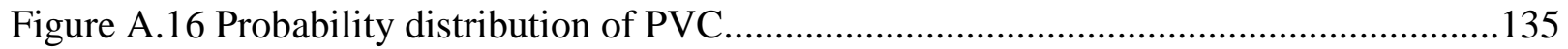

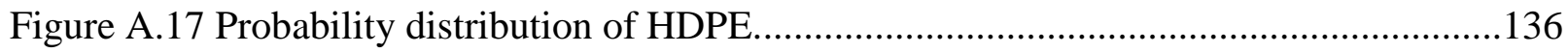

Figure A.18 Probability distribution of PP.............................................................. 136

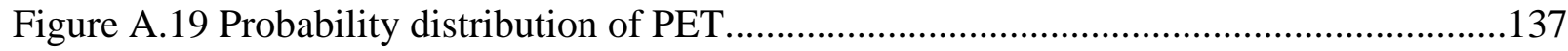

Figure A.20 Probability distribution of CFRP ...........................................................137

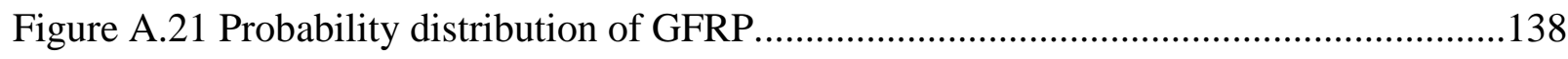

Figure A.22 Probability distribution of cast iron...........................................................138

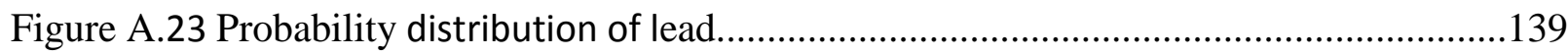

Figure A.24 Probability distribution of steel................................................................139

Figure A.25 Probability distribution of recycled steel.................................................140

Figure A.26 Probability distribution of stainless steel...................................................140 
Figure A.27 Probability distribution of aluminum...................................................... 141

Figure A.28 Probability distribution of recycled aluminum........................................... 141

Figure A.29 Probability distribution of magnesium......................................................142

Figure A.30 Probability distribution of platinum...........................................................142 


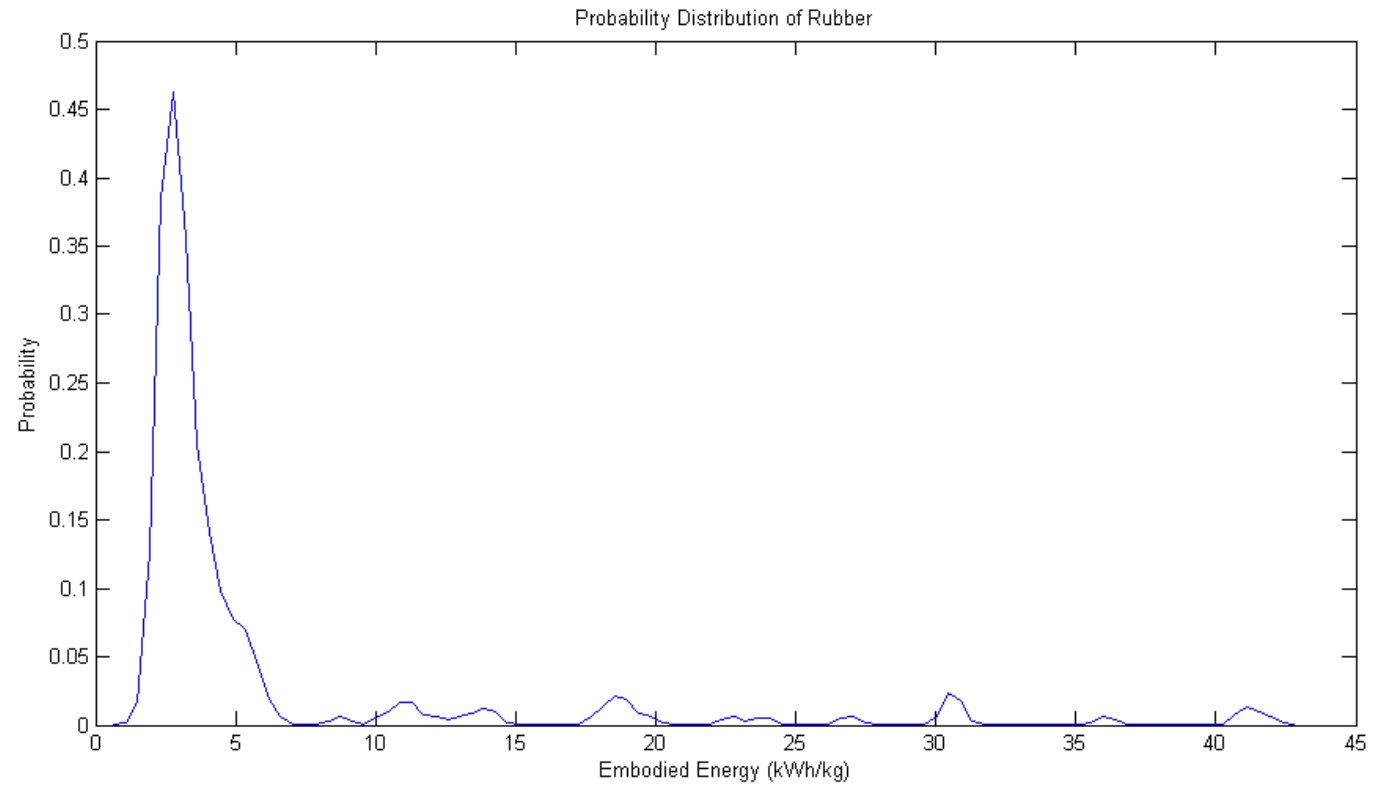

Figure A.1 Probability distribution of rubber.

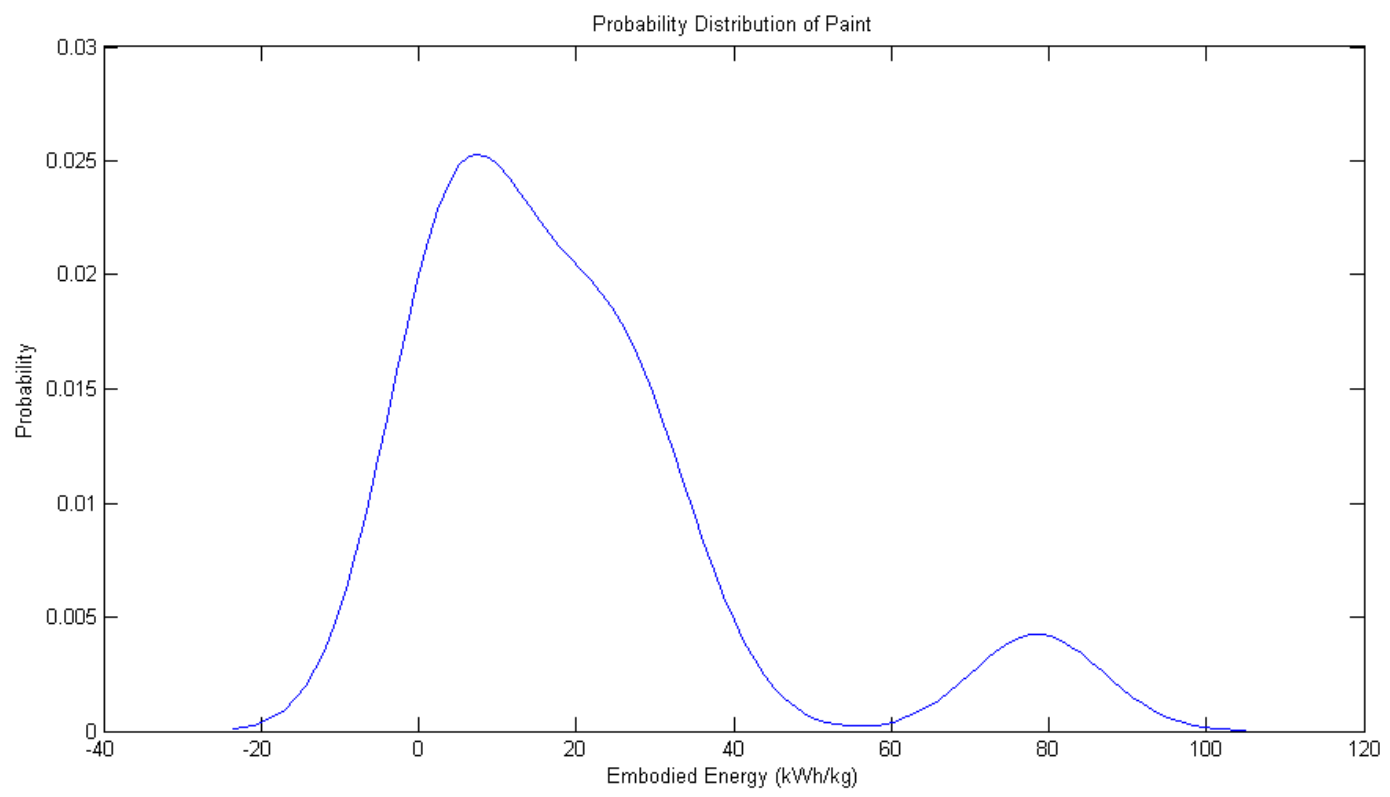

Figure A.2 Probability distribution of paint. 


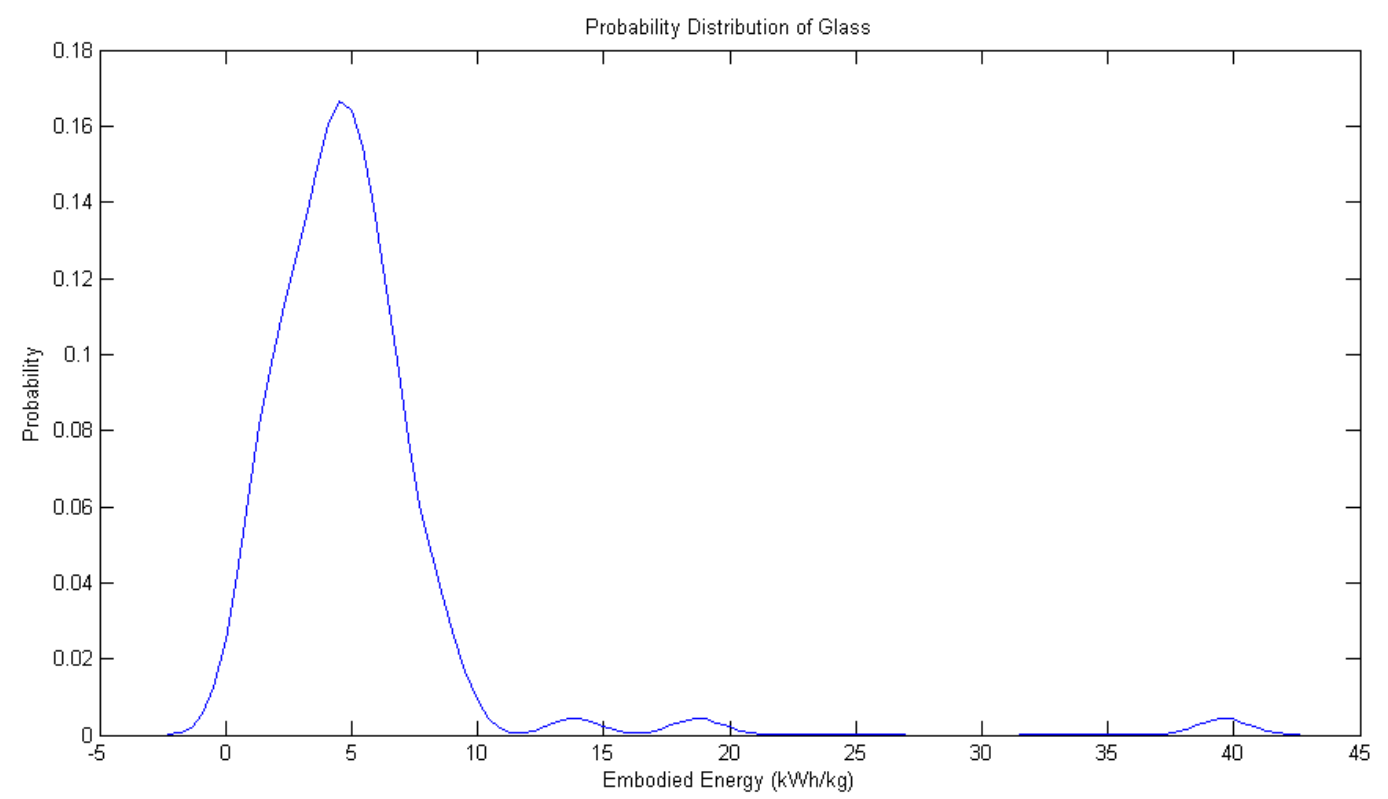

Figure A.3 Probability distribution of glass.

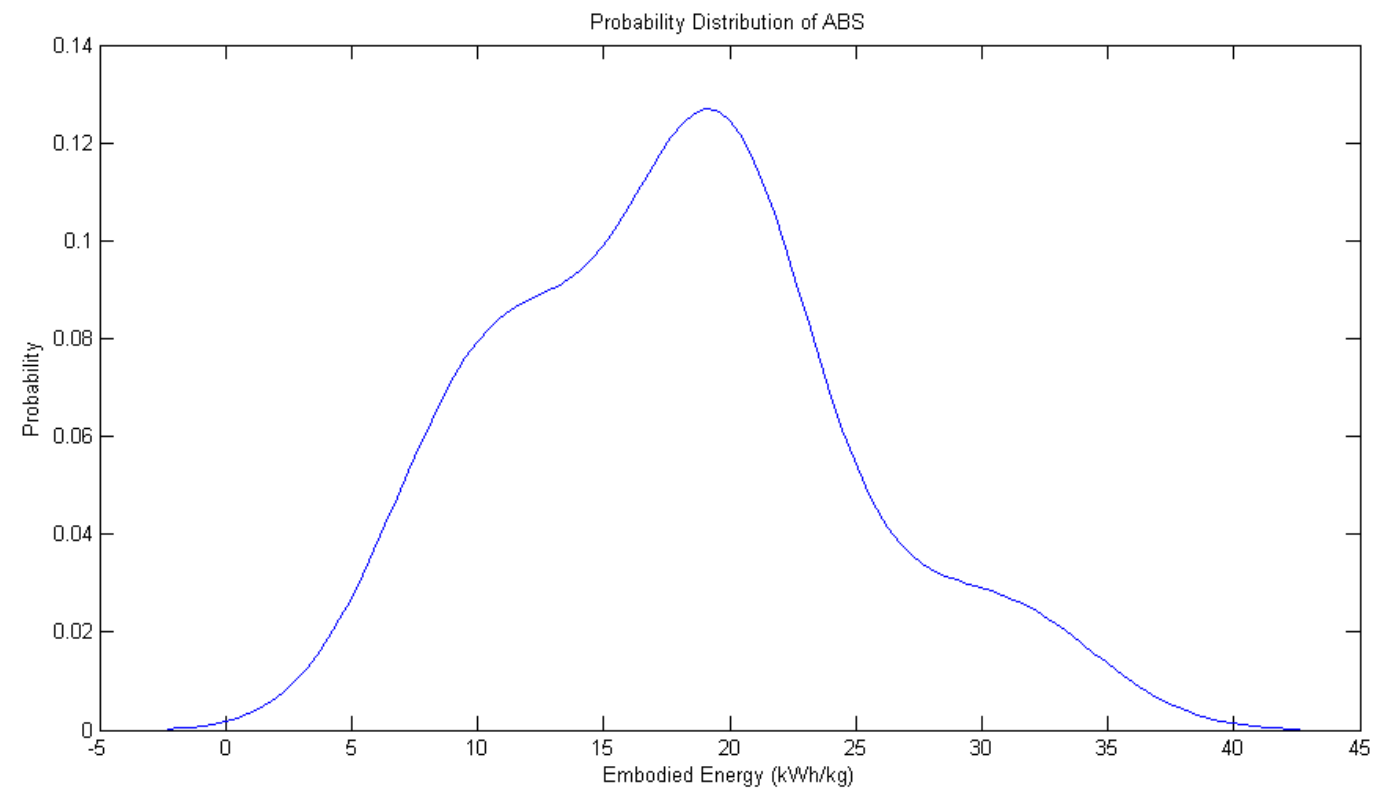

Figure A.4 Probability distribution of ABS. 


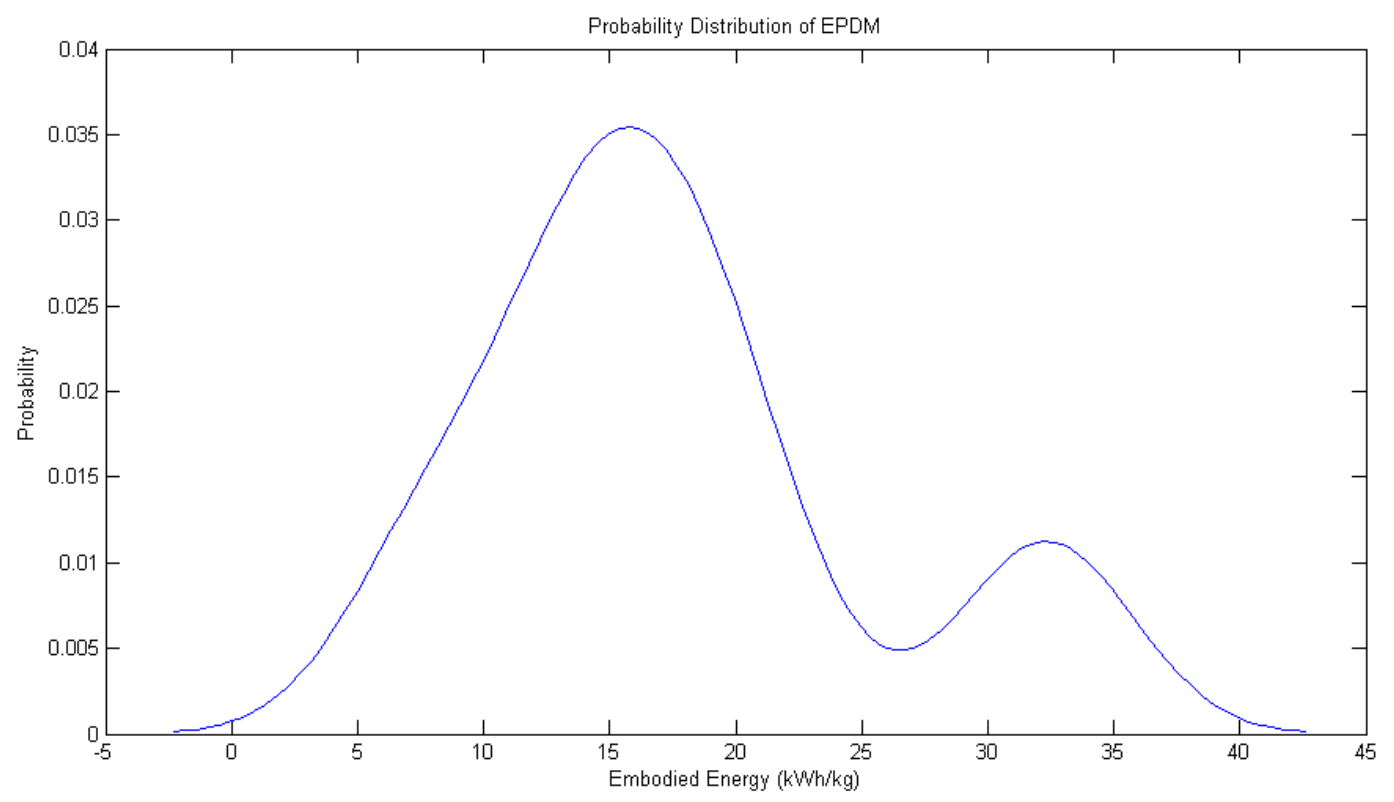

Figure A.5 Probability distribution of EPDM.

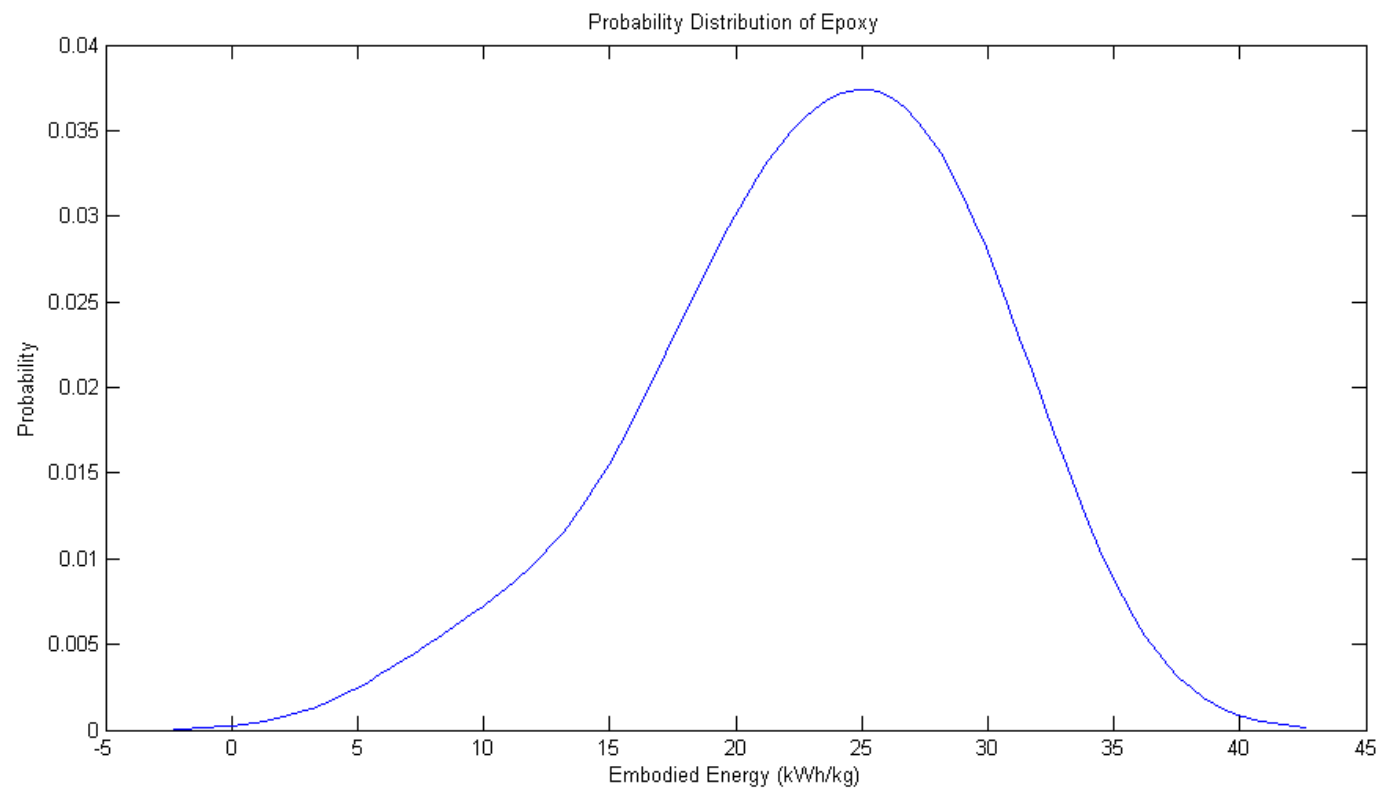

Figure A.6 Probability distribution of epoxy. 


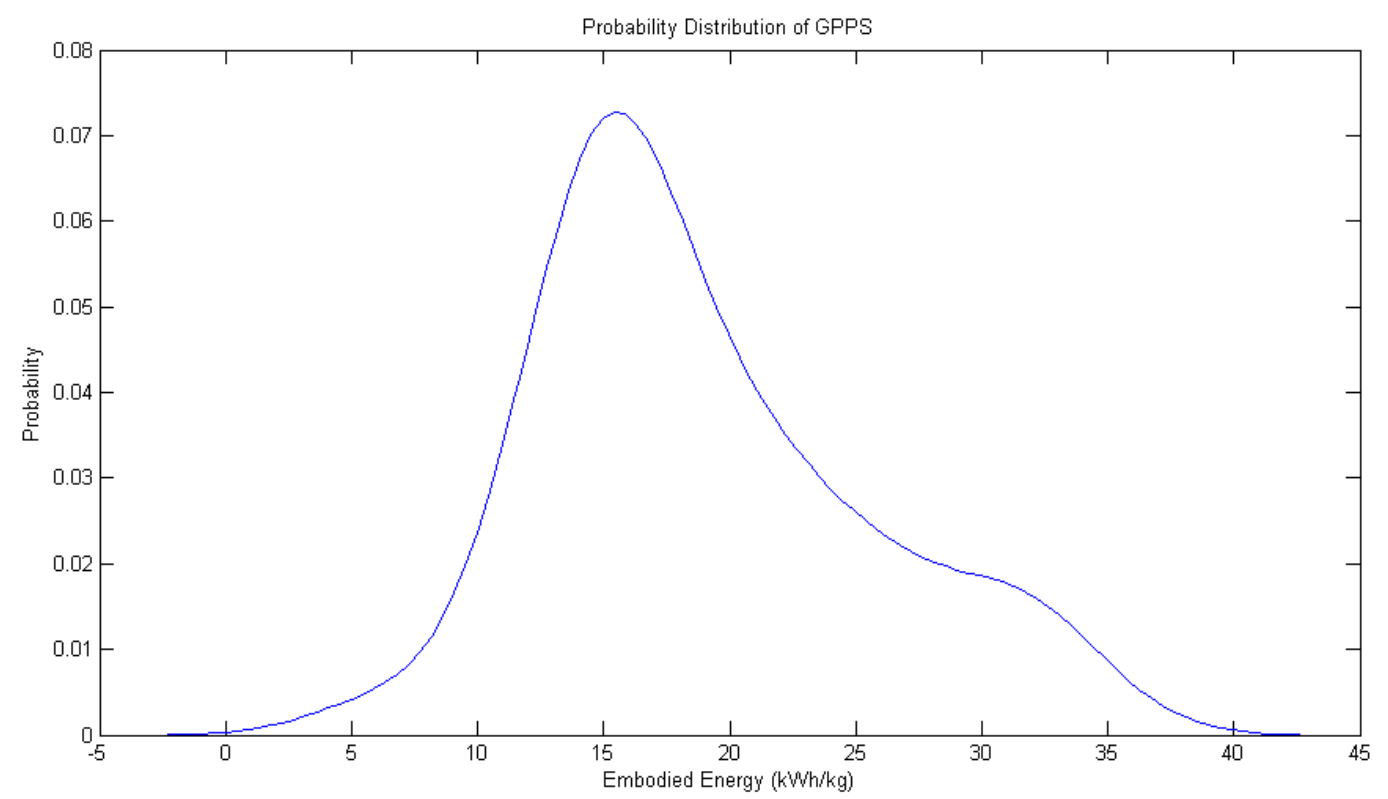

Figure A.7 Probability distribution of GPPS.

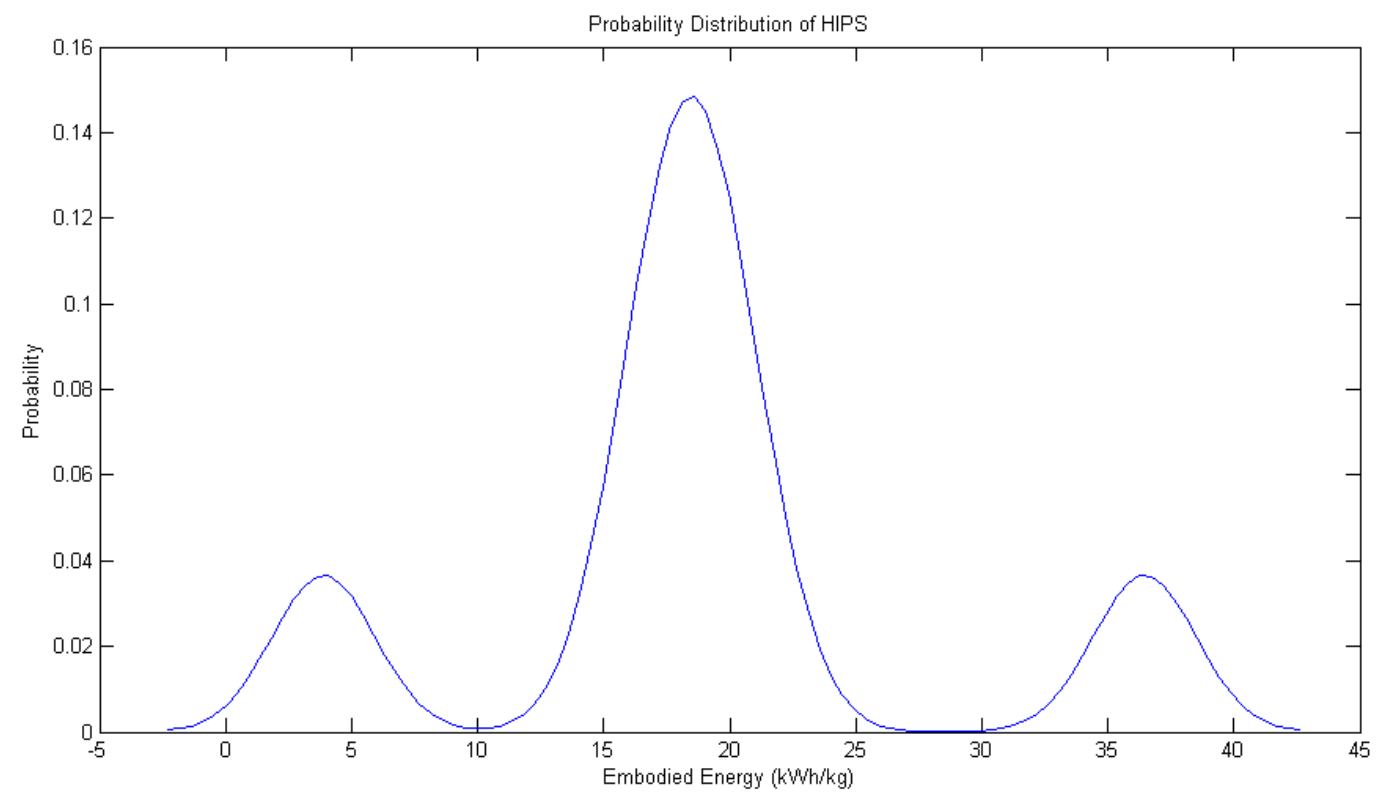

Figure A.8 Probability distribution of HIPS. 


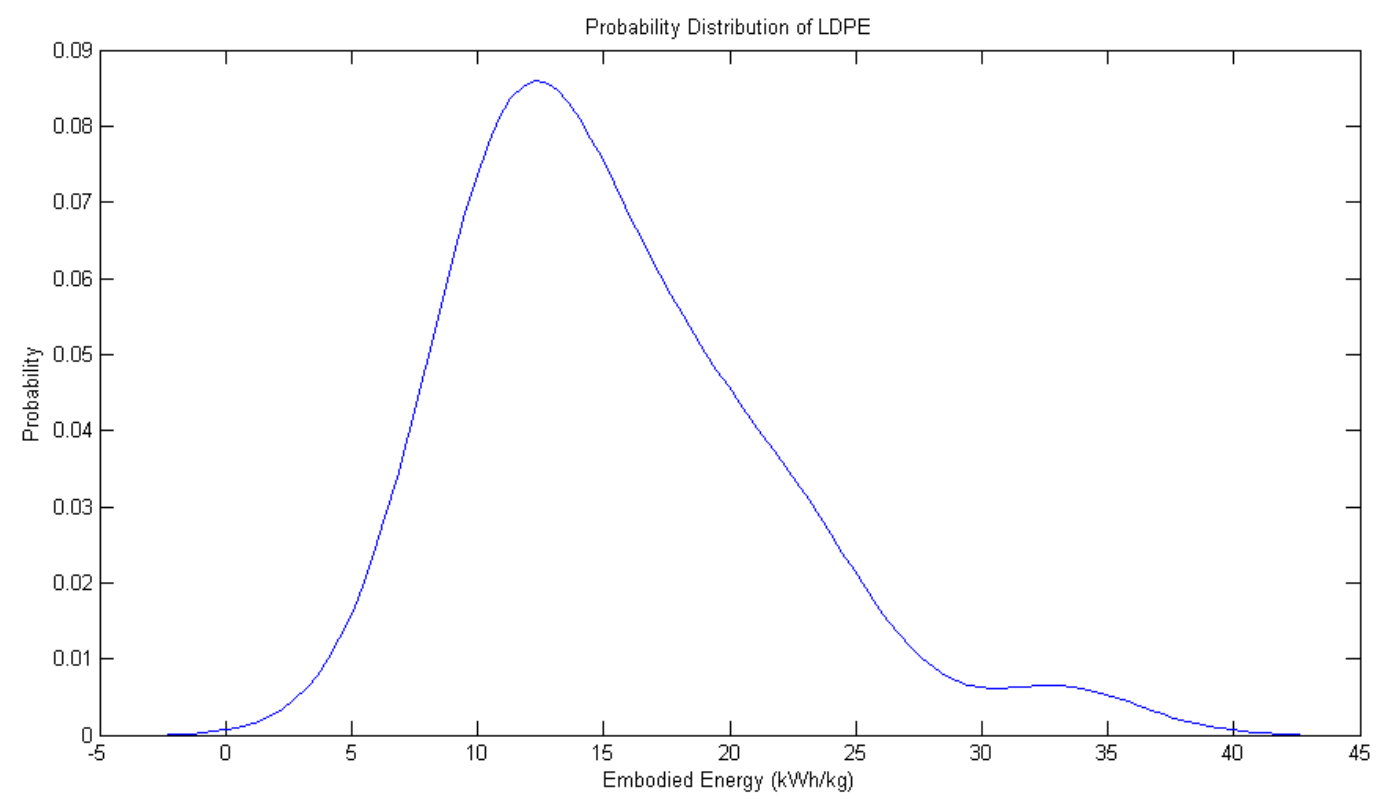

Figure A.9 Probability distribution of LDPE.

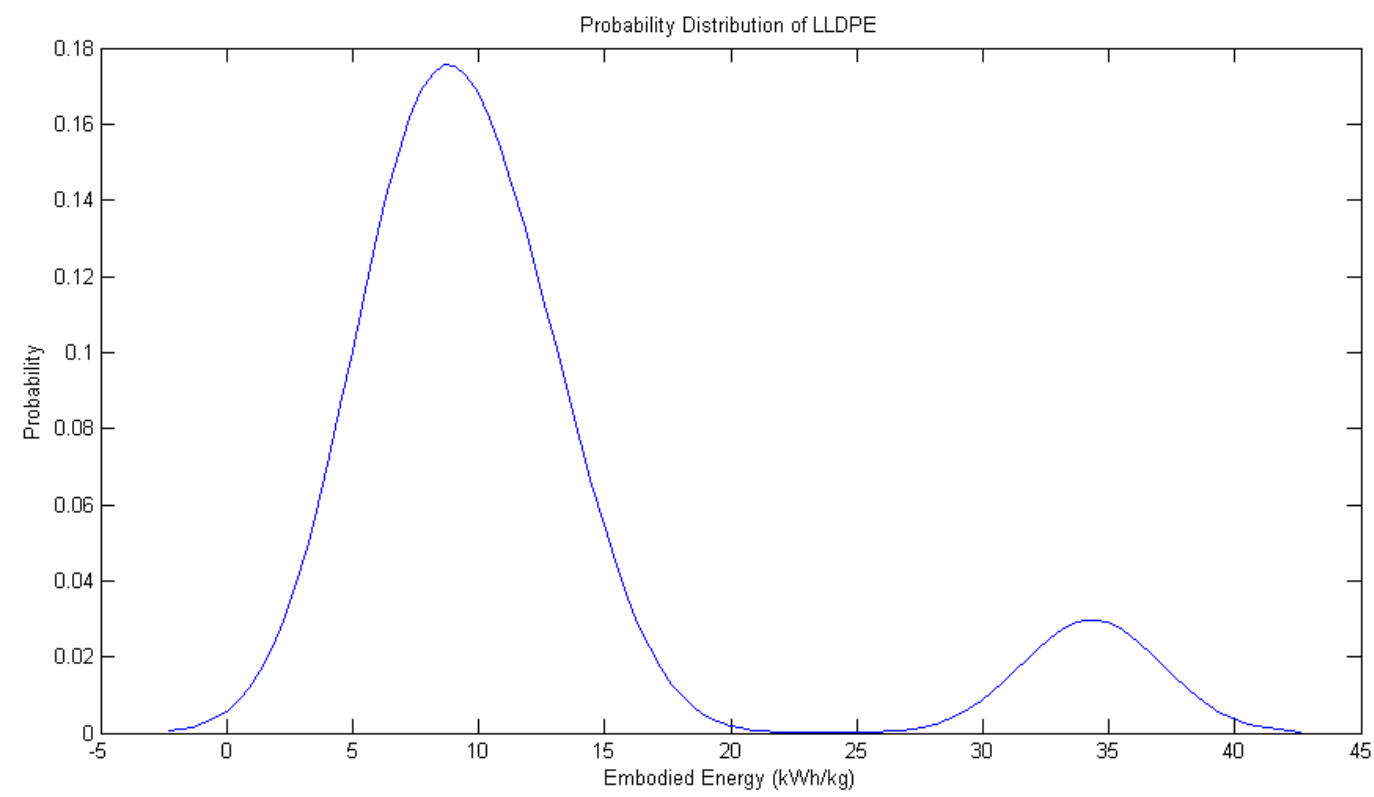

Figure A.10 Probability distribution of LLDPE. 


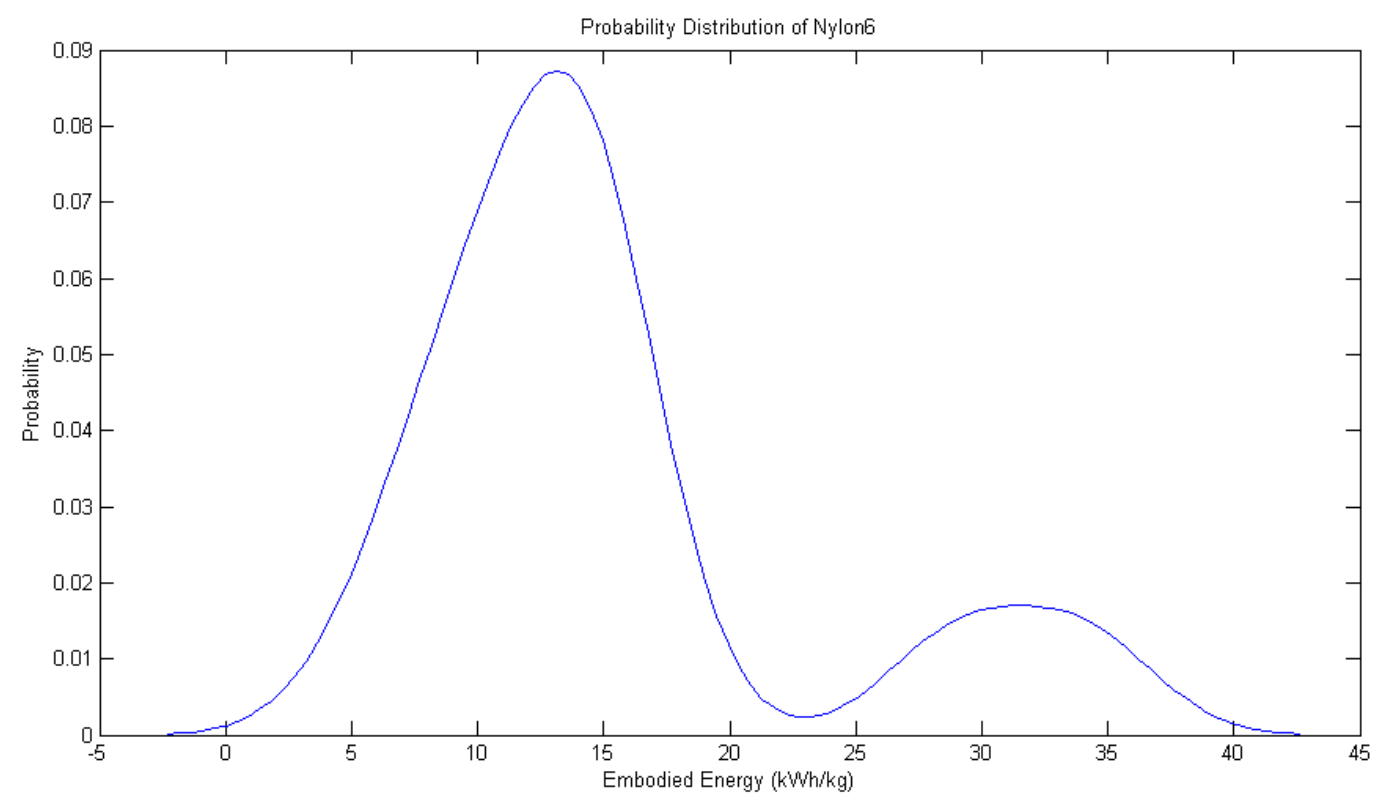

Figure A.11 Probability distribution of Nylon6.

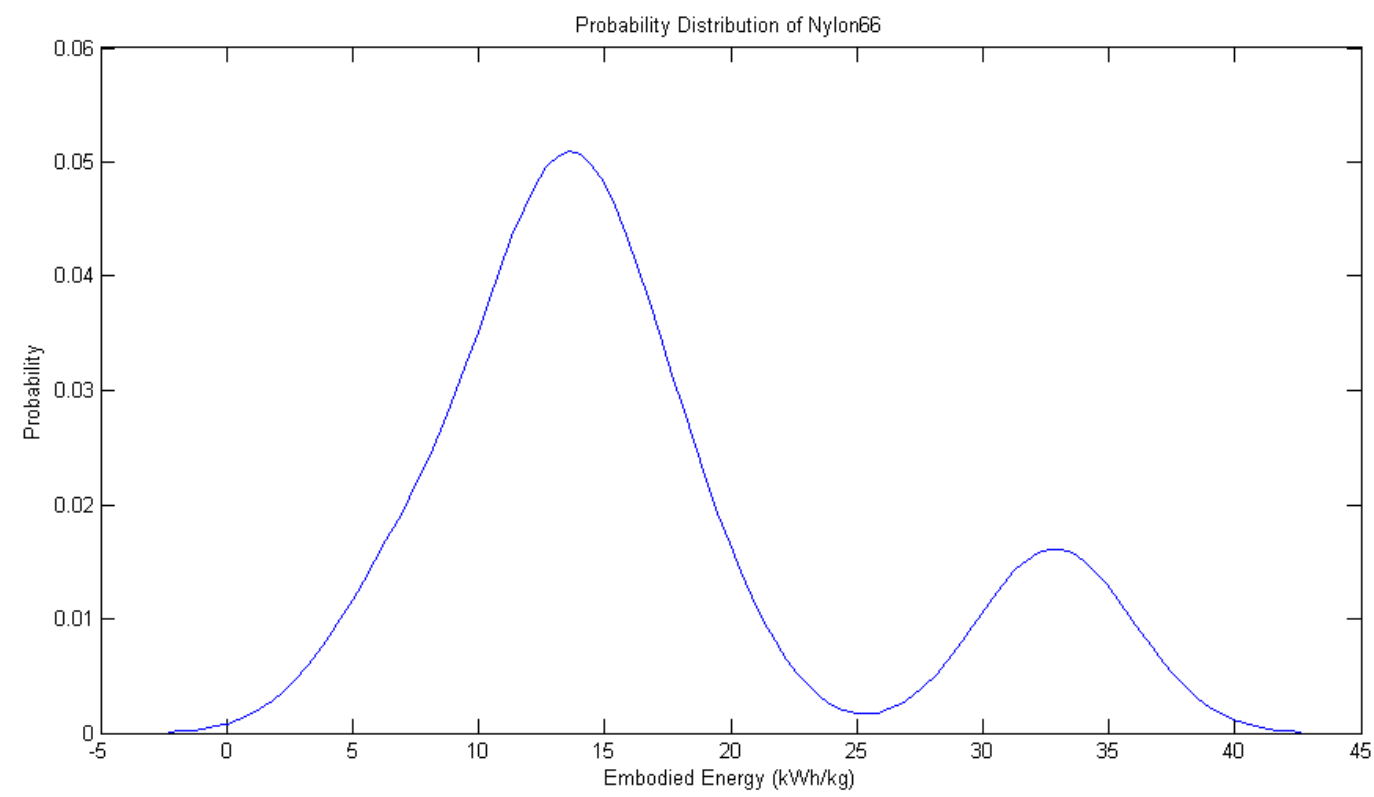

Figure A.12 Probability distribution of Nylon66. 


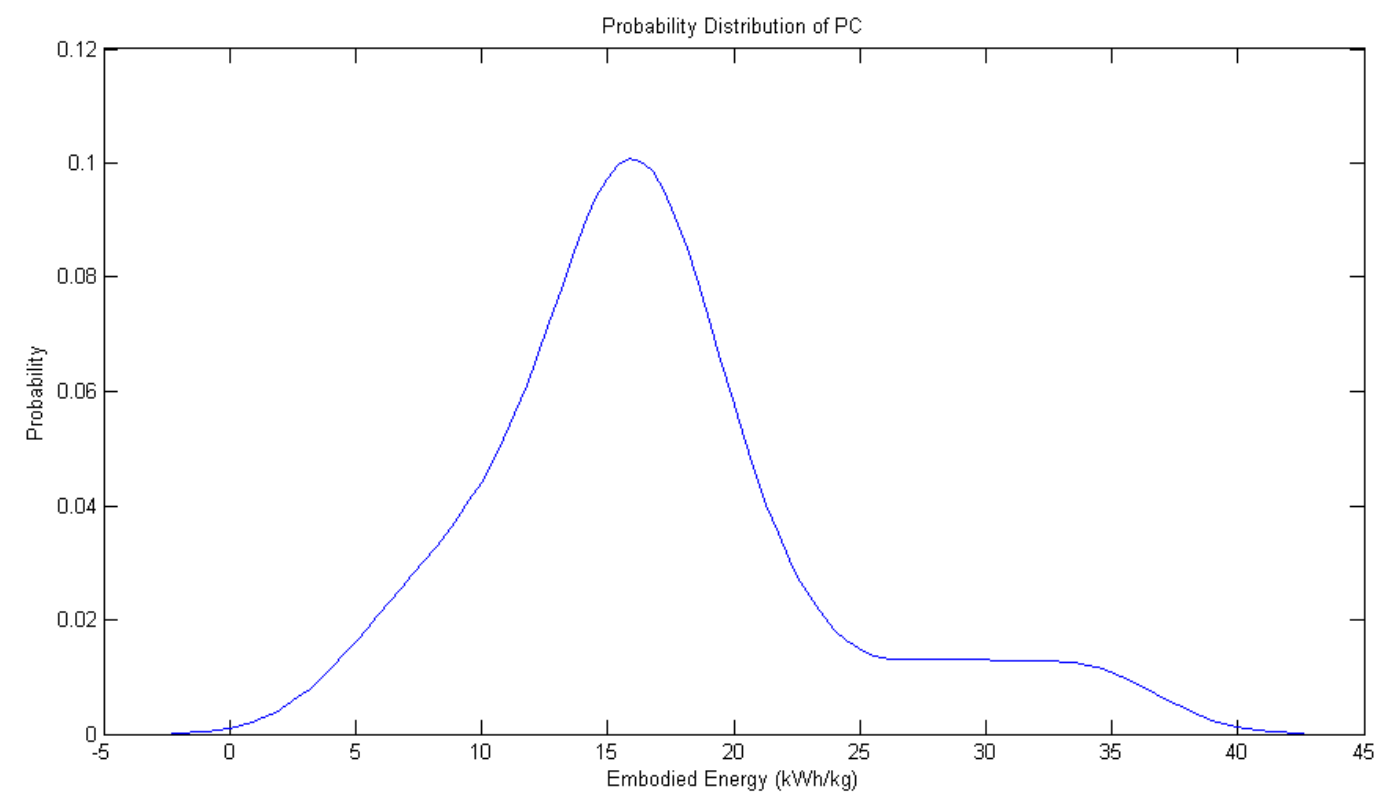

Figure A.13 Probability distribution of PC.

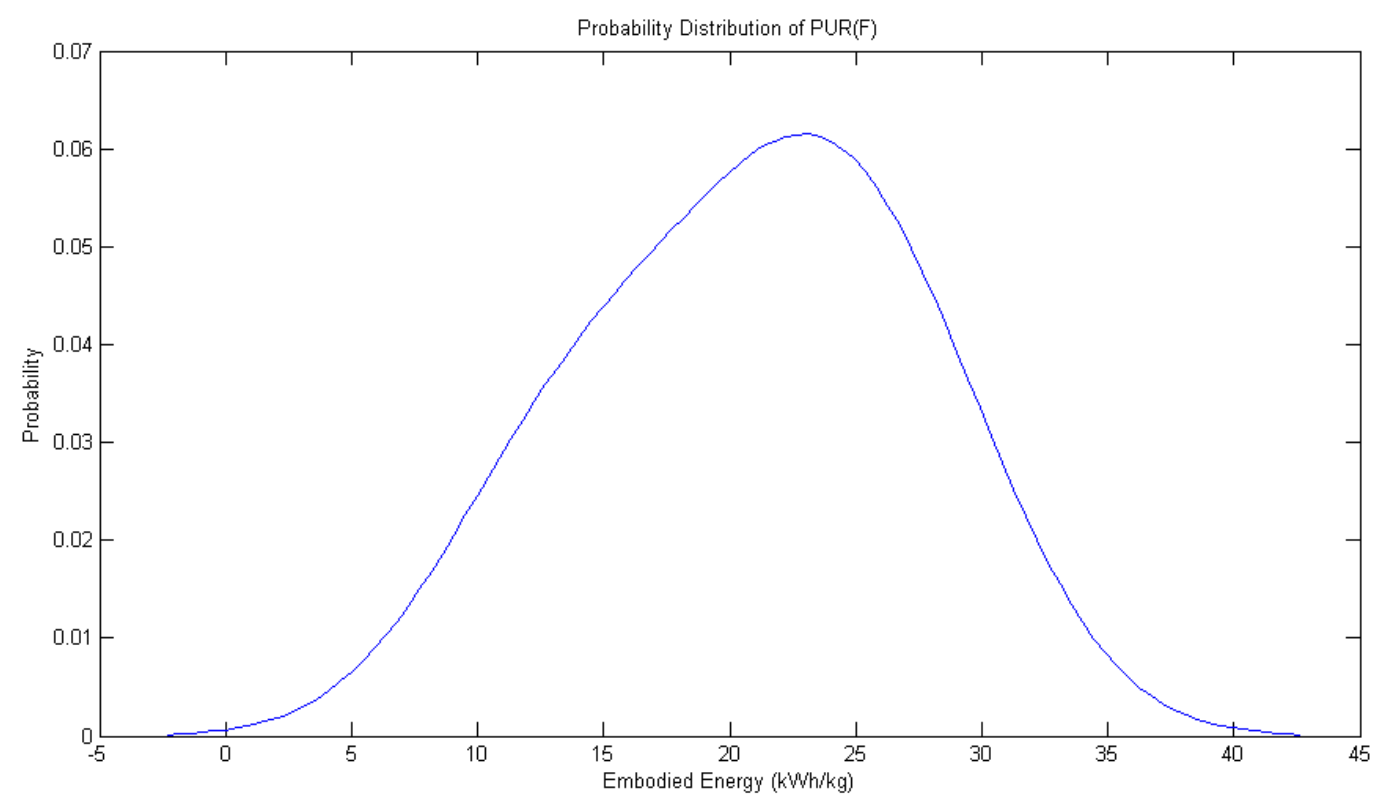

Figure A.14 Probability distribution of PUR(F). 


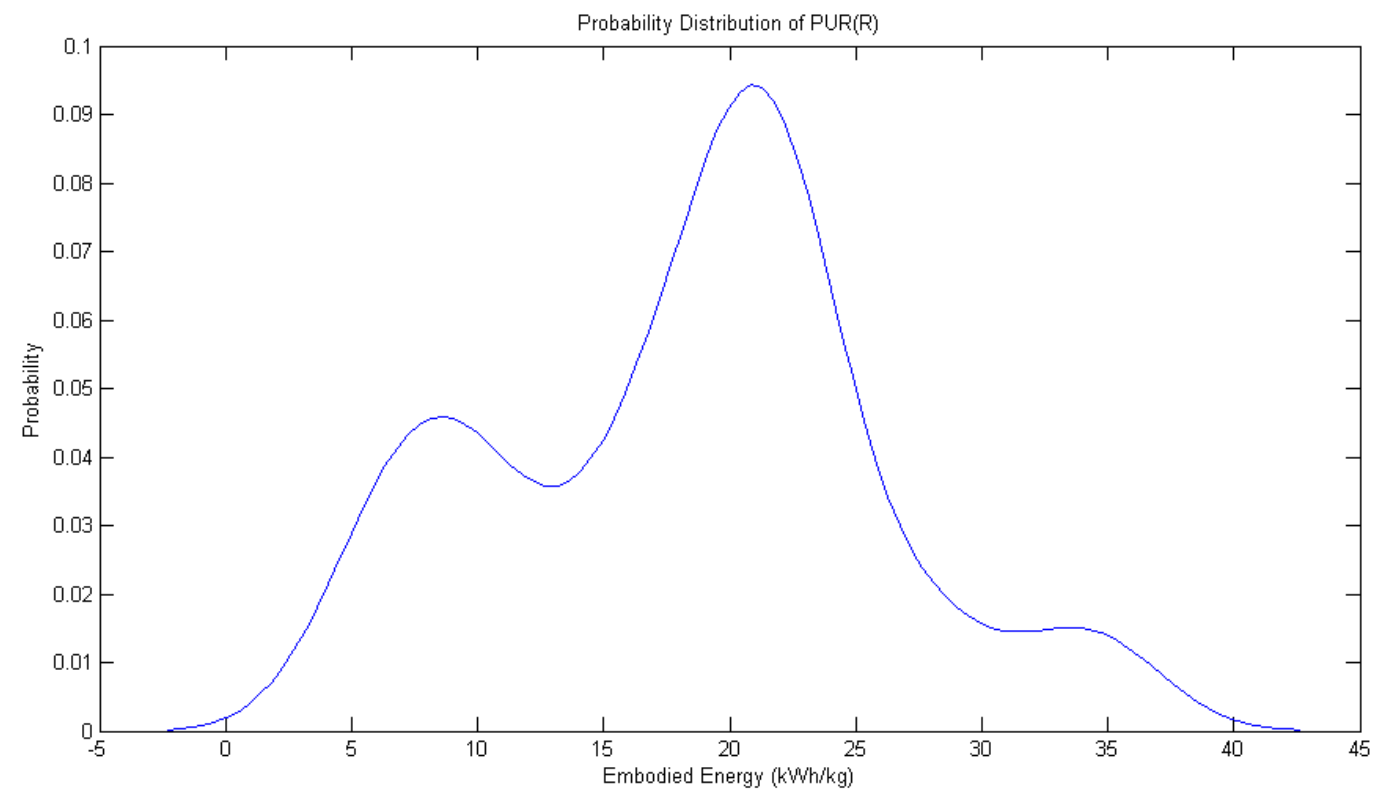

Figure A.15 Probability distribution of PUR(F).

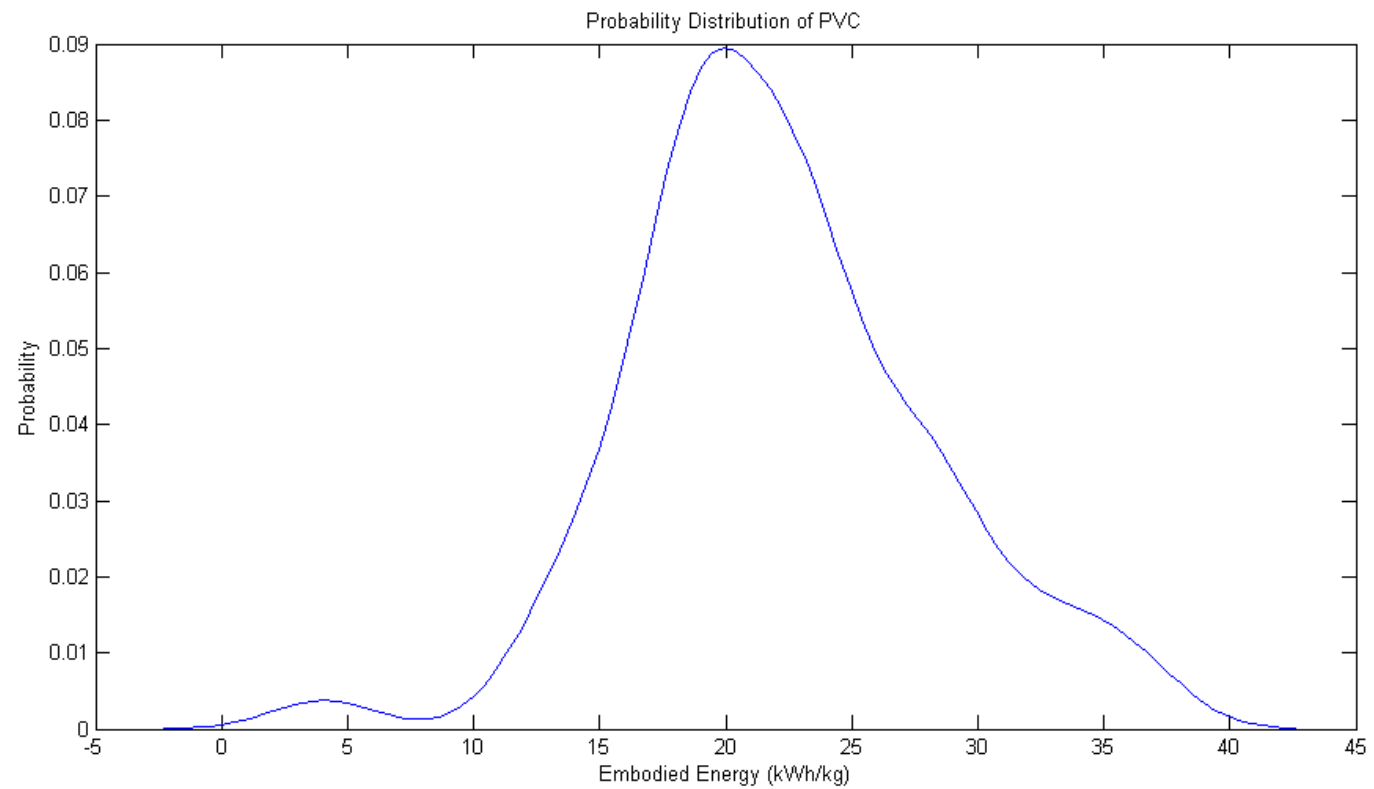

Figure A.16 Probability distribution of PVC. 


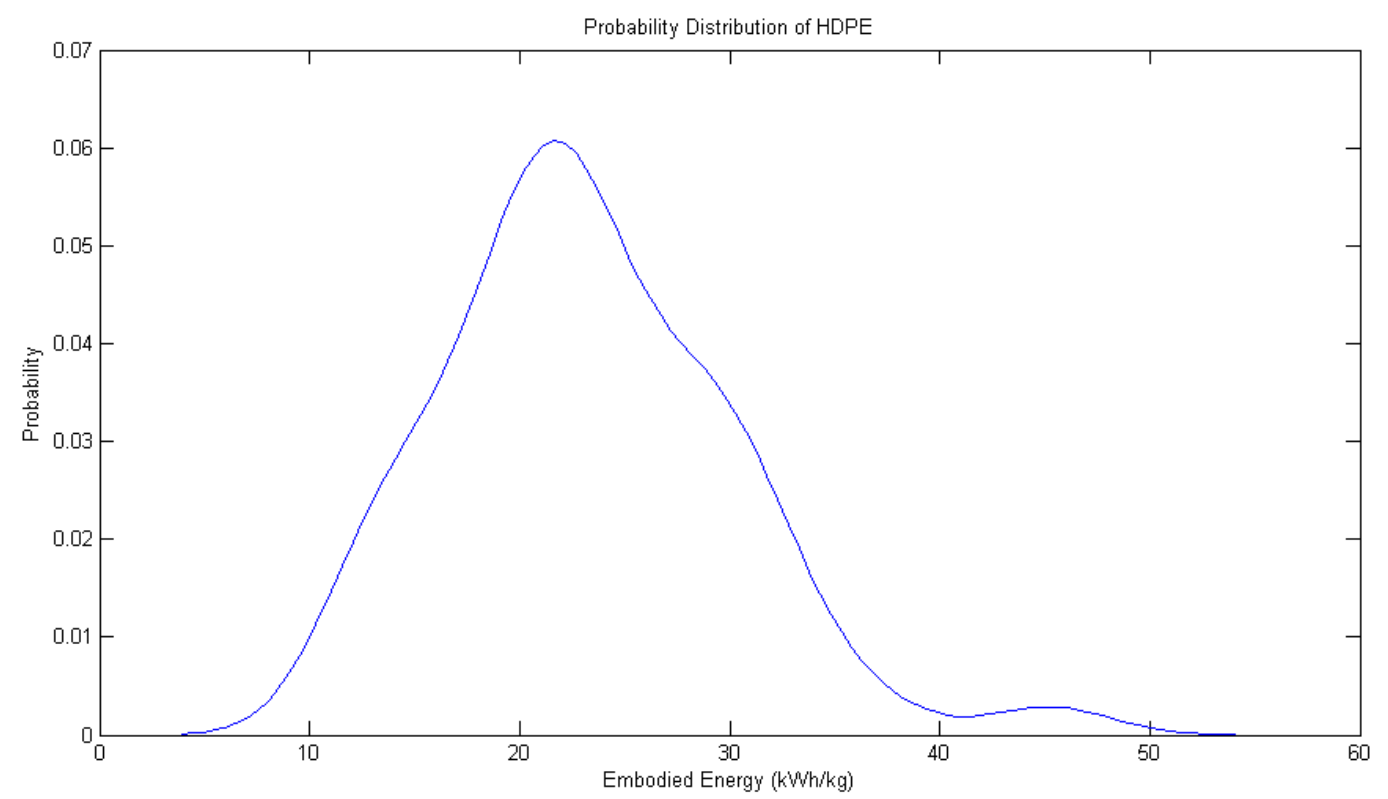

Figure A.17 Probability distribution of HDPE.

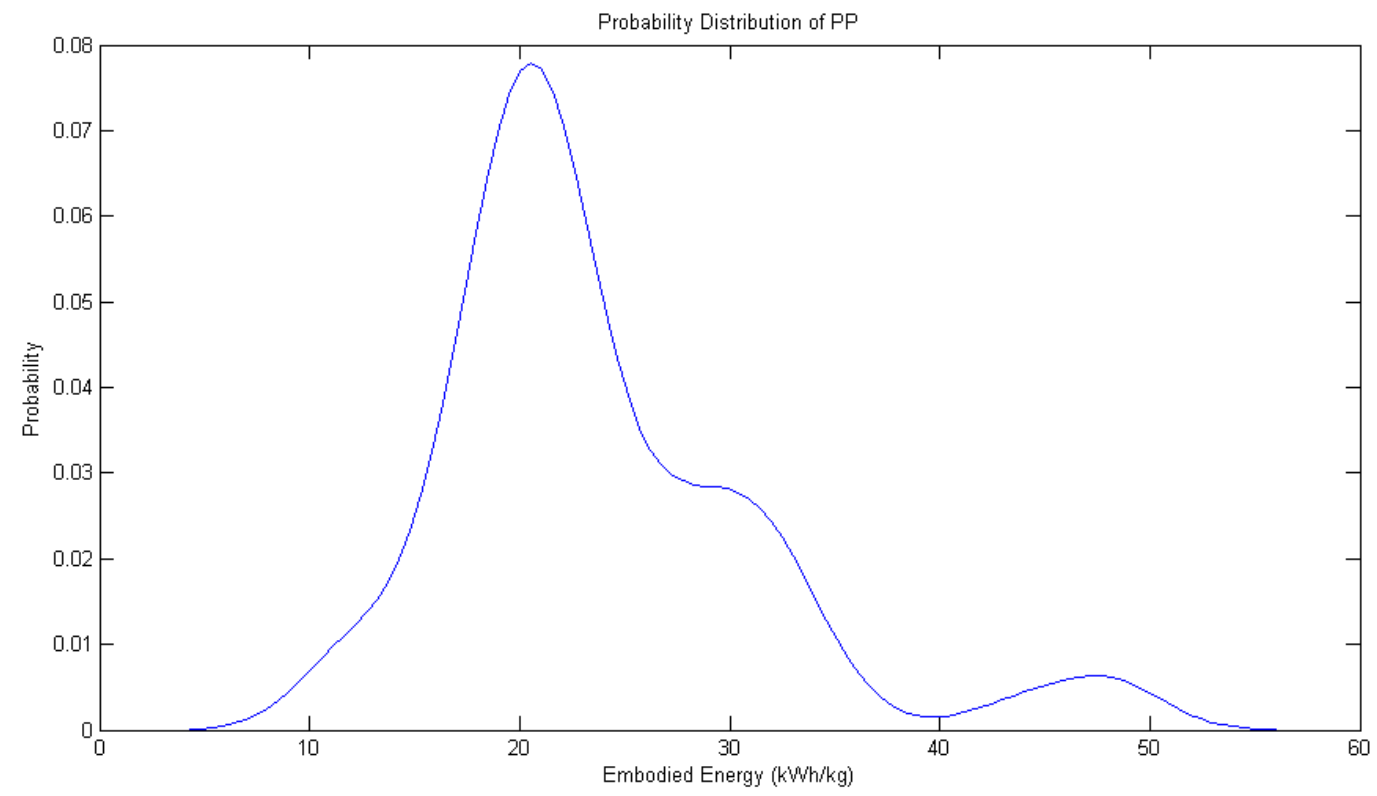

Figure A.18 Probability distribution of PP. 


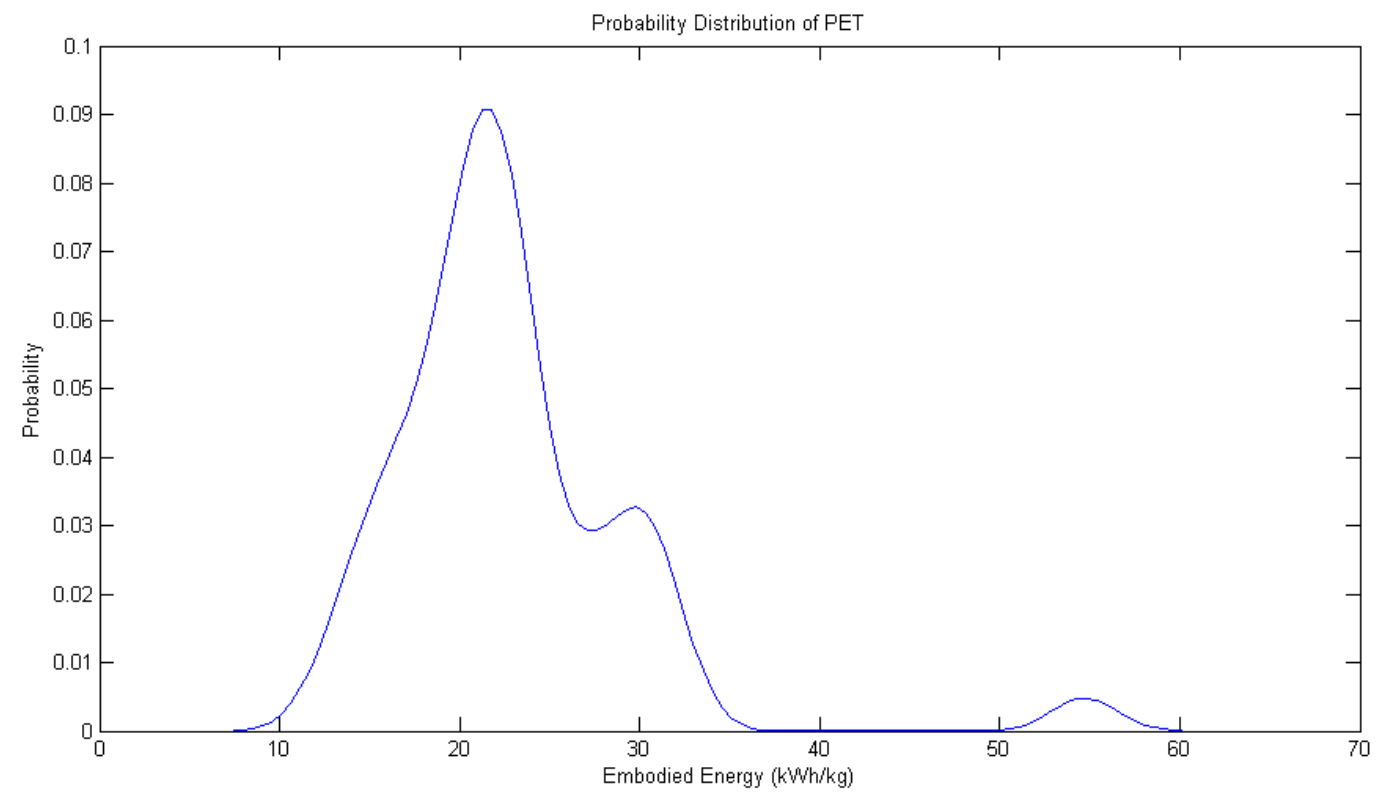

Figure A.19 Probability distribution of PET.

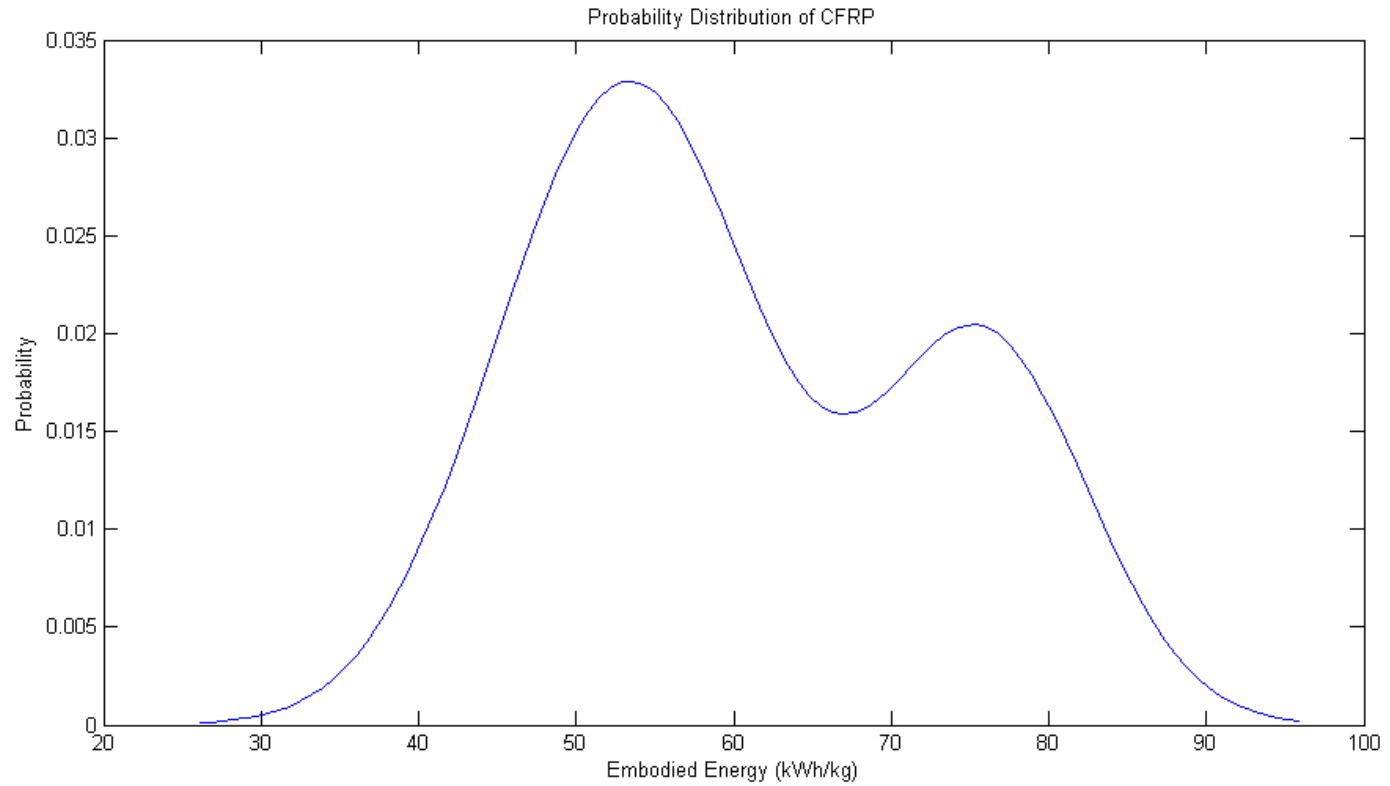

Figure A.20 Probability distribution of CFRP. 


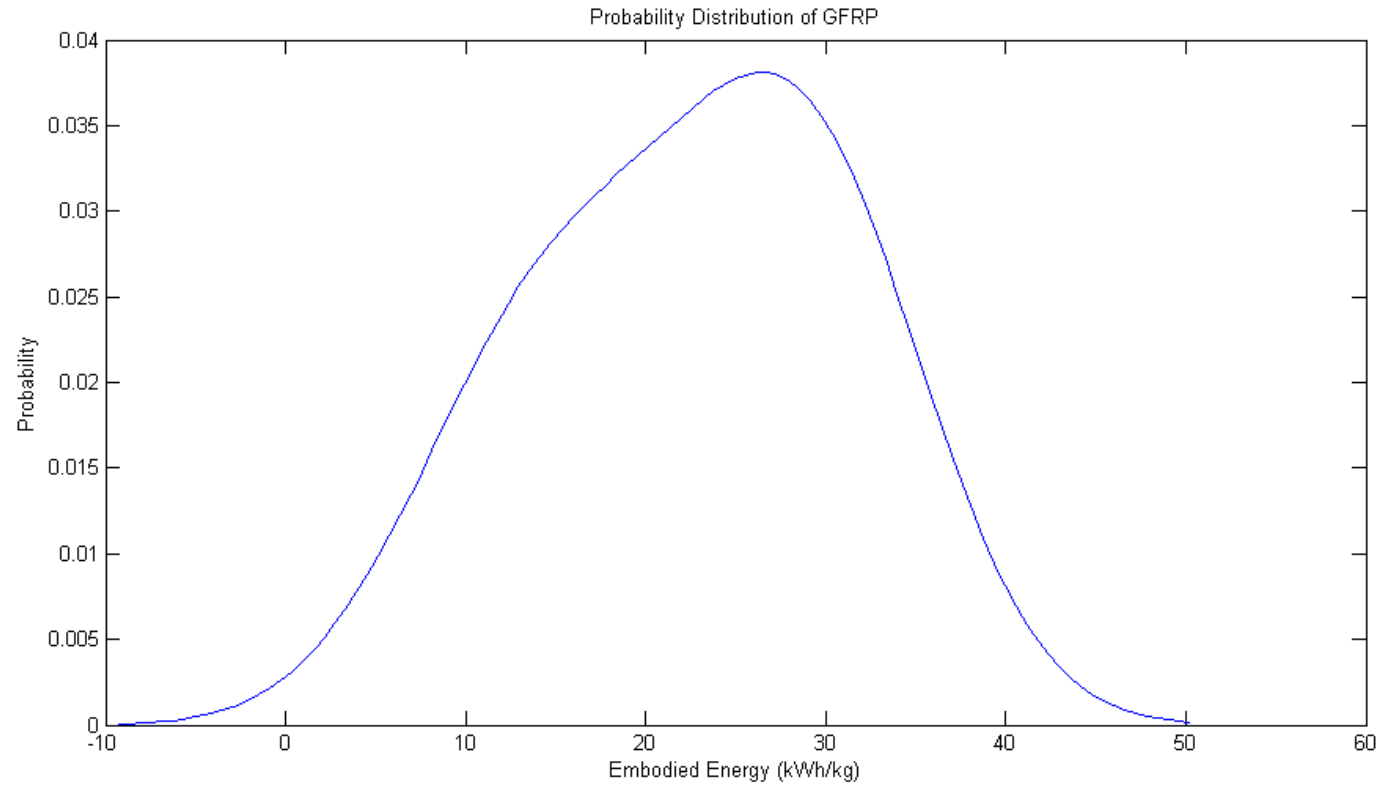

Figure A.21 Probability distribution of GFRP.

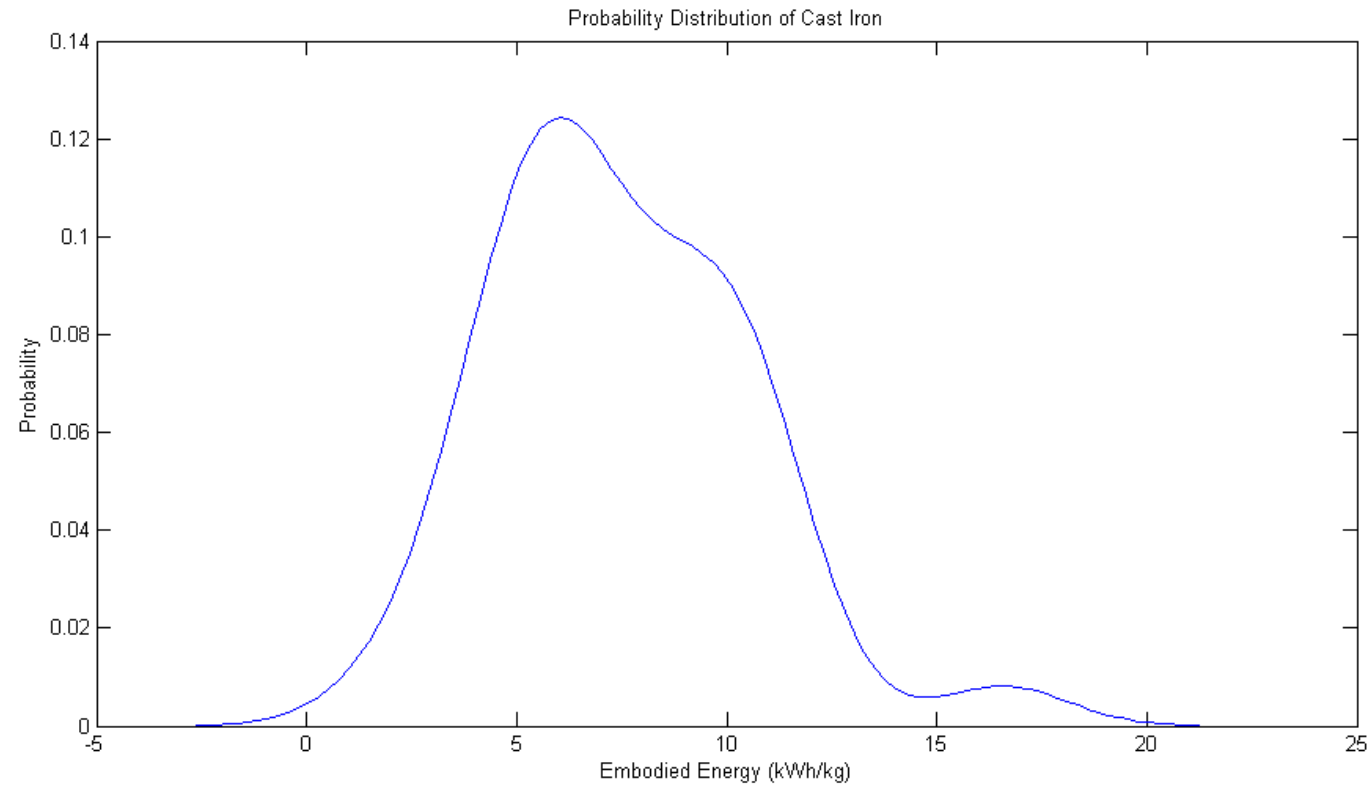

Figure A.22 Probability distribution of cast iron. 


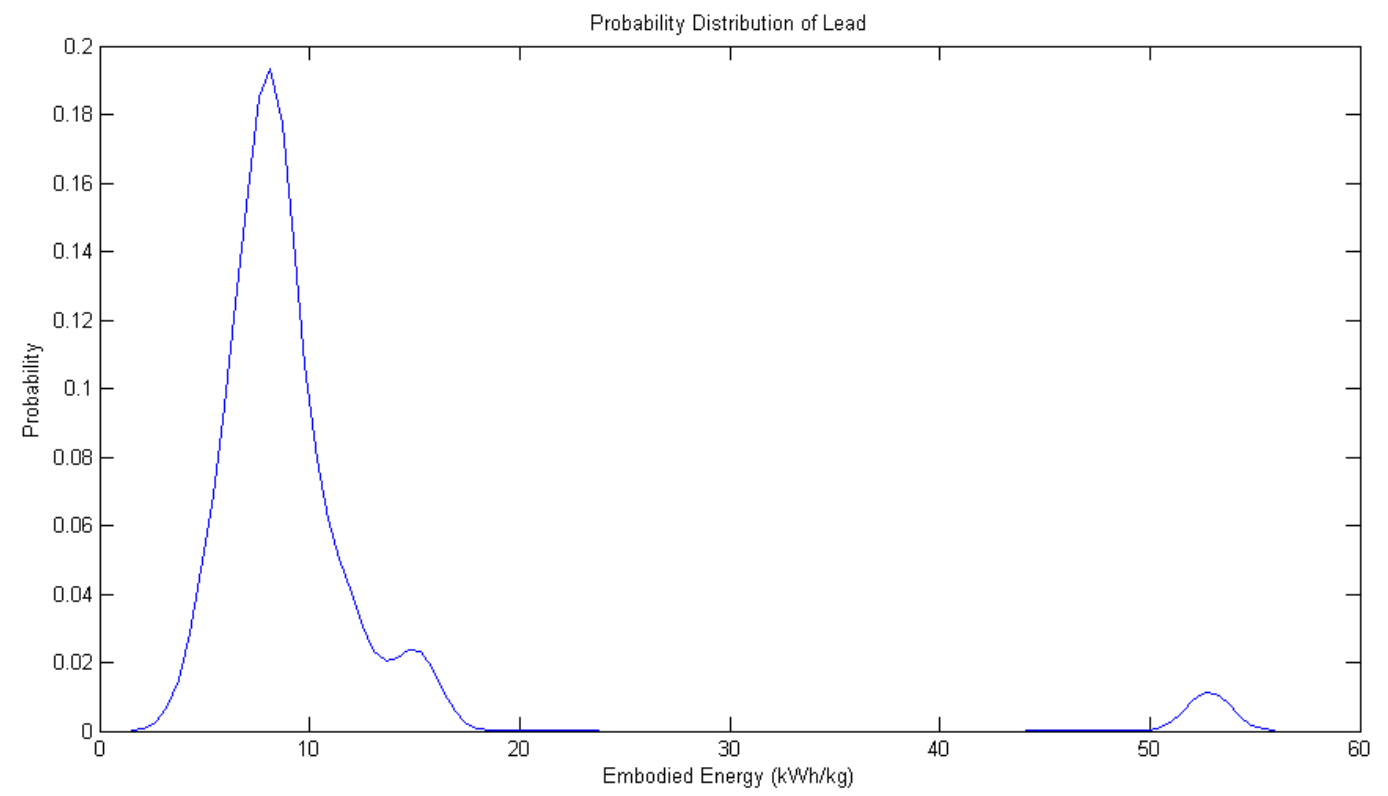

Figure A.23 Probability distribution of lead.

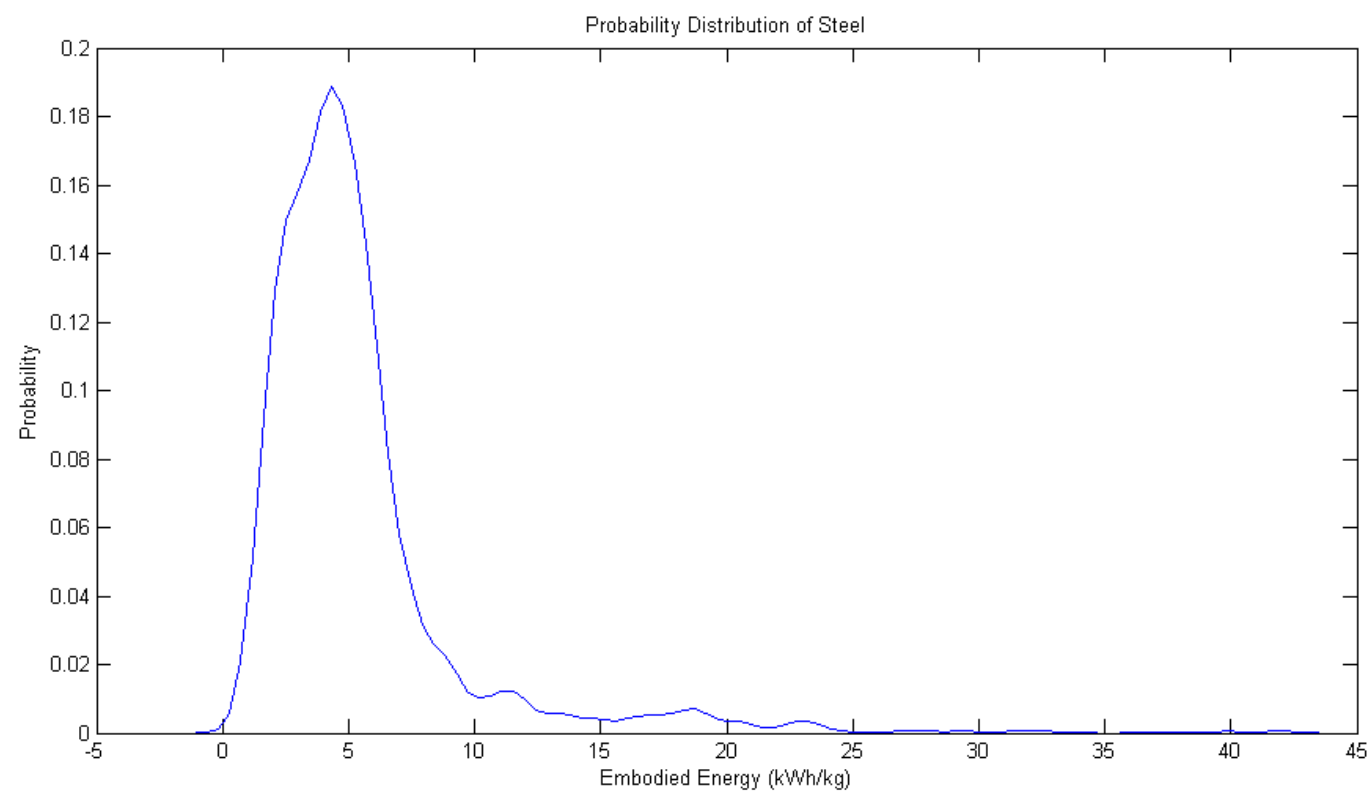

Figure A.24 Probability distribution of steel. 


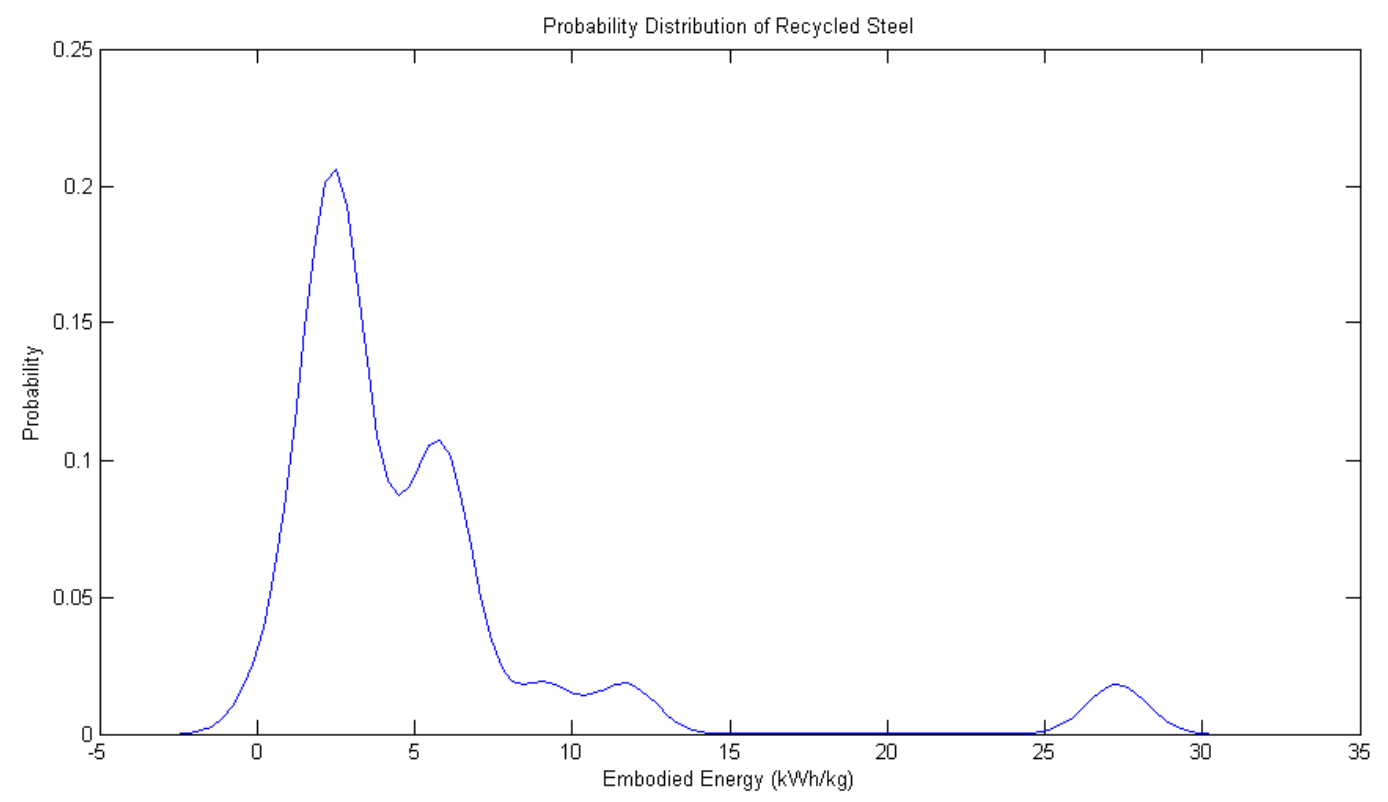

Figure A.25 Probability distribution of recycled steel.

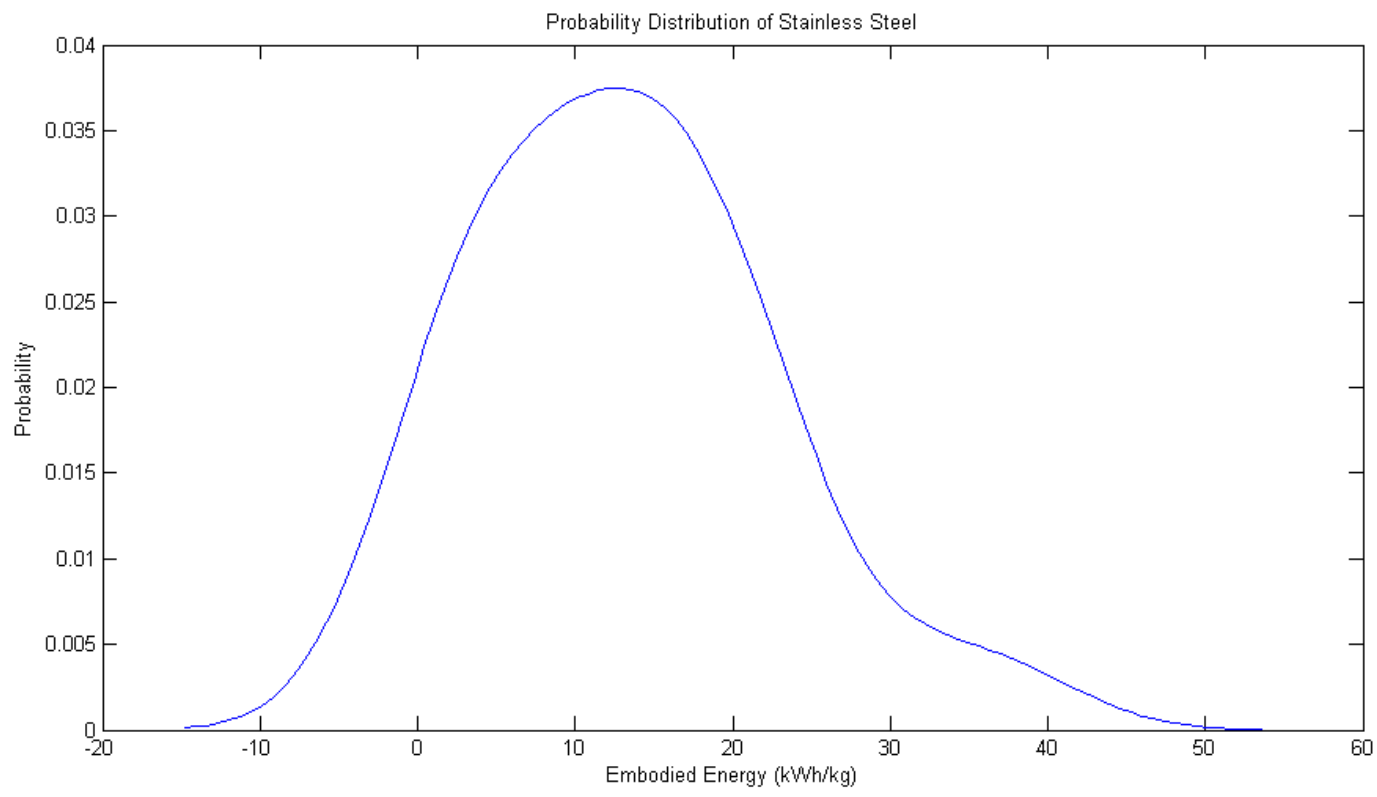

Figure A.26 Probability distribution of stainless steel. 


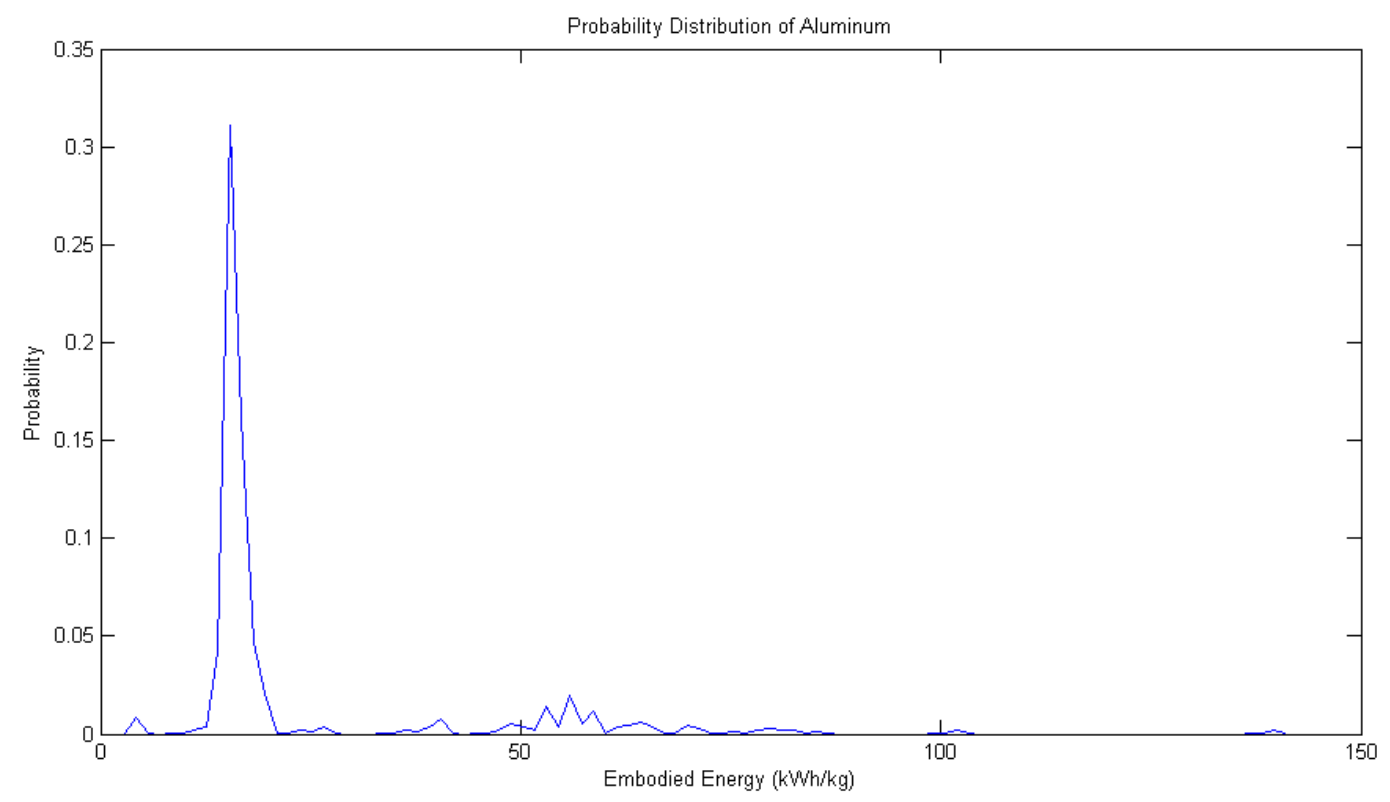

Figure A.27 Probability distribution of aluminum

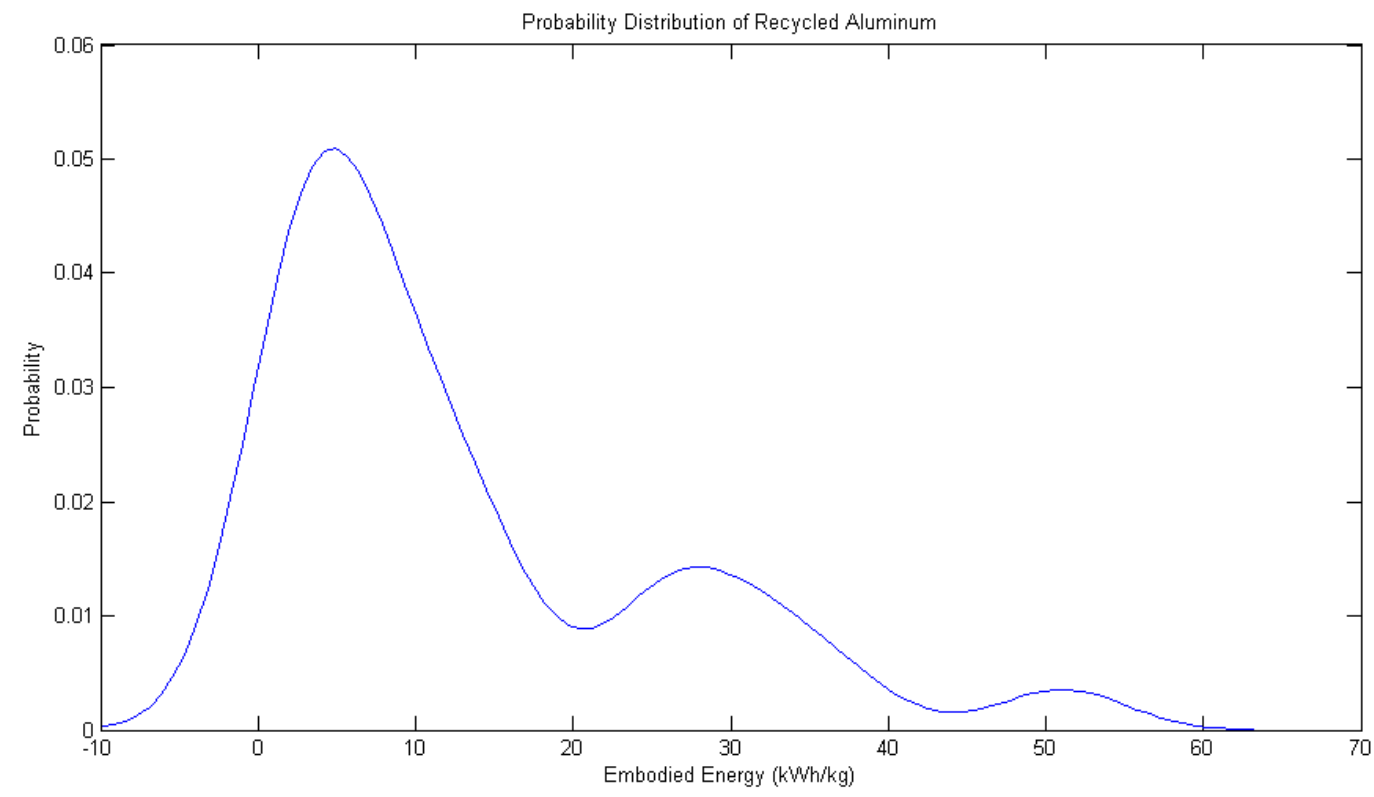

Figure A.28 Probability distribution of recycled aluminum. 


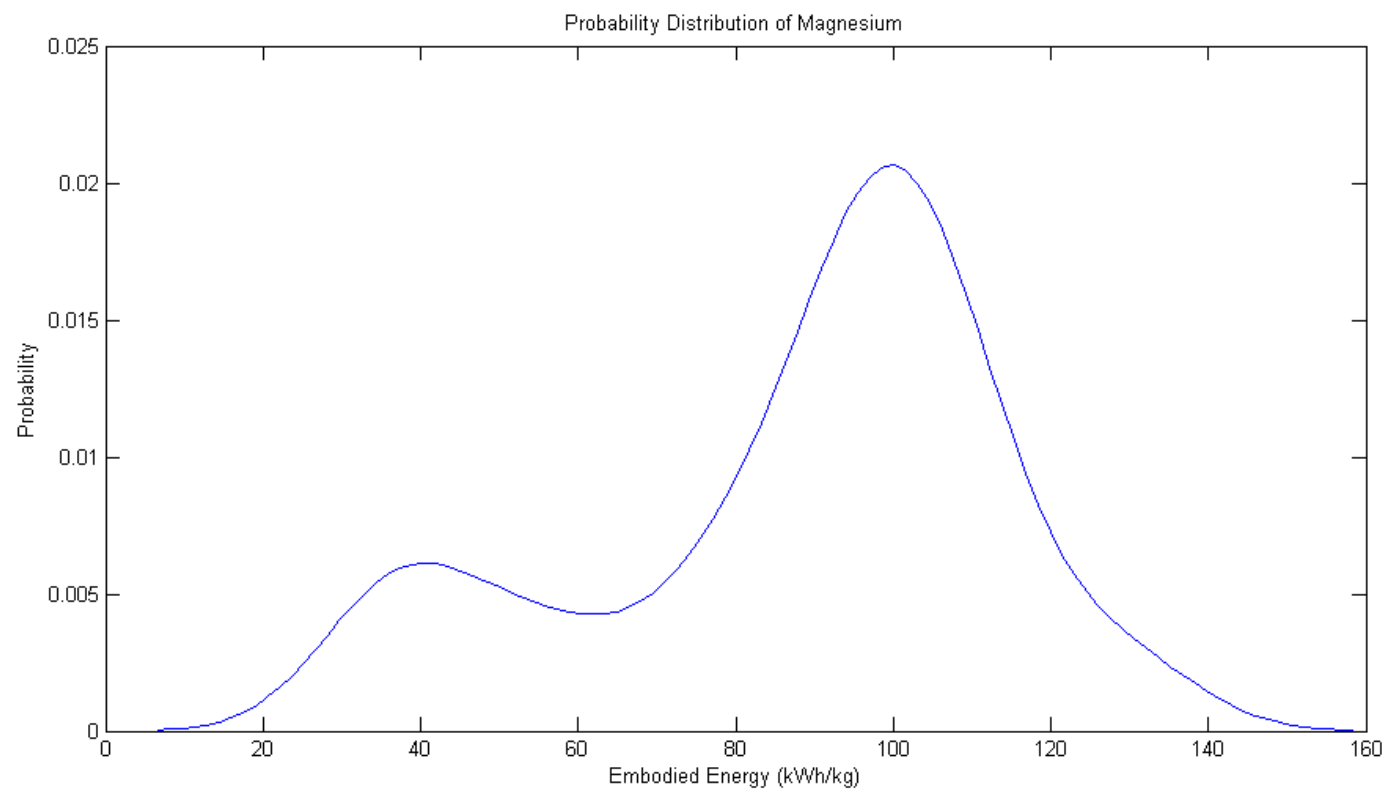

Figure A.29 Probability distribution of magnesium.

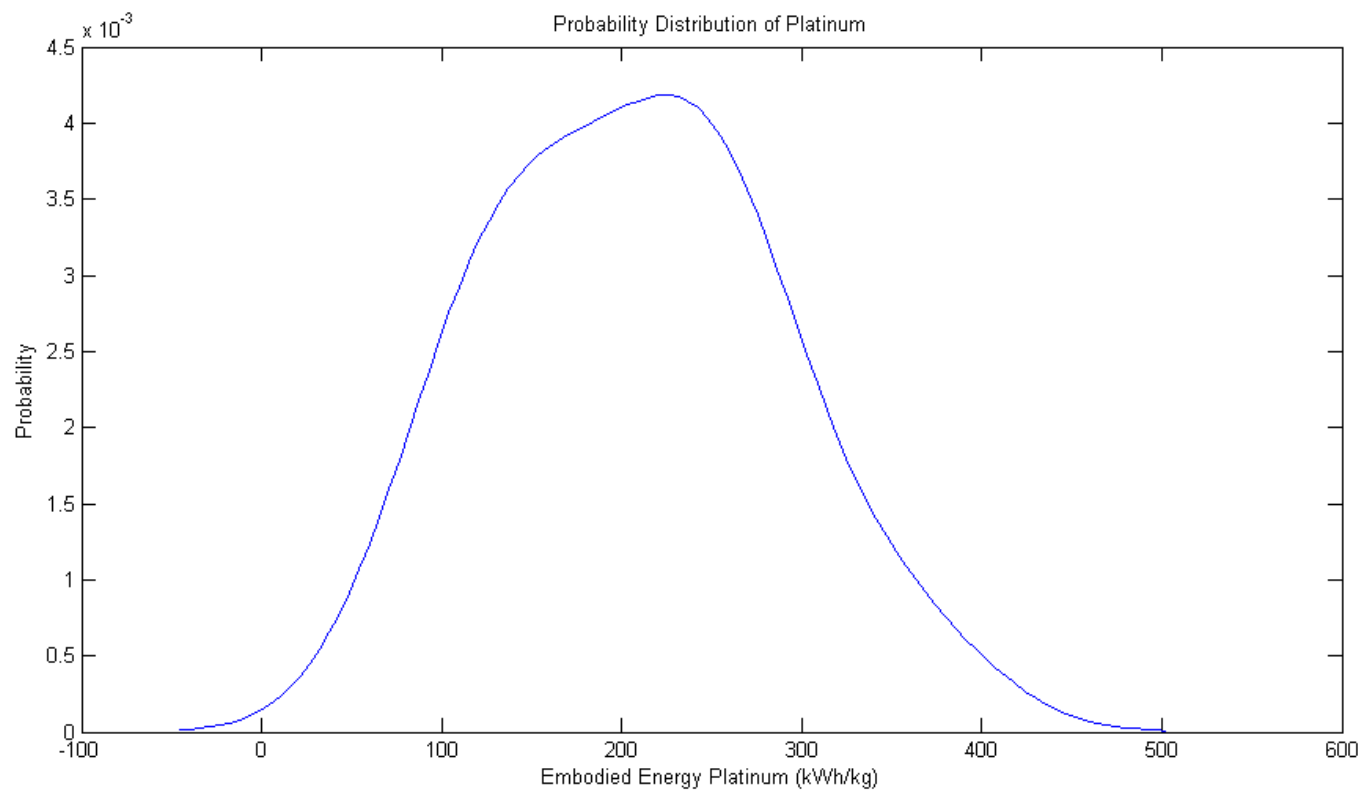

Figure A.30 Probability distribution of platinum. 


\section{APPENDIX B-DATA REFERENCES}

Abeysundra, U.G.Y., Babel, S., Gheewala, S., and Sharp, A. (2007a). Environmental, economic and social analysis of materials for doors and windows in Sri Lanka. Building and Environment, 42(5), 2141-2149.

Abeysundra, U.G.Y., Babel, S., and Gheewala, S. (2007b). A decision making matrix with life cycle perspective of materials for roofs in Sri Lanka. Materials and Design, 28(9), 2478-2487.

AkzoNobel. (2012). AkzoNobel report 2011. Amsterdam, the Netherlands: AkzoNobel.

Alcorn, J.A., and Baird, G. (1996). Embodied energy analysis of New Zealand building materials-methods and results. In Treloar, G., Fay, R., and Tucker, S. (Eds.), Proceedings of the embodied energy: the current state of play seminar, November 28-29, 1996, Geelong, Australia (pp.61-71). Geelong, Australia: Deakin University.

Alcorn, A. (2001). Embodied energy and $\mathrm{CO}_{2}$ coefficients for $\mathrm{NZ}$ building materials. Wellington, New Zealand: Centre for Building Performance Research.

Alcorn, A. (2003). Embodied energy and $\mathrm{CO}_{2}$ coefficients for $\mathrm{NZ}$ building materials. Wellington, New Zealand: Centre for Building Performance Research.

Aluminum Association. (2011). Aluminum: the element of sustainability. Arlington, VA: The Aluminum Association.

Andrady, A.L. (2003a). An environmental primer. In Andrady, A.L. (Ed.), Plastics and the environment (pp. 3-61). Hoboken, NJ: John Wiley and Sons.

Andrady, A.L. (2003b). Common plastics materials. In Andrady, A.L. (Ed.), Plastics and the environment (pp. $77-$ 121). Hoboken, NJ: John Wiley and Sons.

Aquafil Group. (2011). Environmental product declaration for Econyl@ nylon textile filament yarn. Trento, Italy: Aquafil Group.

Ashby, M.F. (1999). Materials selection in mechanical design, $2^{\text {nd }}$ edition. Oxford, UK: Butterworth-Heinemann.

Ashby, M.F. (2005). Materials selection in mechanical design, $3^{\text {rd }}$ edition. Oxford, UK: Butterworth-Heinemann.

Ashby, M.F. (2011). Materials selection in mechanical design, $4^{\text {th }}$ edition. Oxford, UK: Butterworth-Heinemann.

Ayres, R.U., Ayres, L.W., and Råde, I. (2002). The life cycle of copper, its co-products and by-products. London, UK: International Institute for Environment and Development.

Badino, V., Baldo, G.L., and Legarth, J. (1997). LCA approach to the automotive glass recycling. Journal of Environmental Sciences, 9(2), 208-214.

Baird, G., Alcorn, A., and Haslam, P. (1997). The energy embodied in building materials — updated New Zealand coefficients and their significance. IPENZ Transactions, 24(1), 46-54.

Baird, G., and Chan, S.A. (1983). Energy cost of houses and light construction buildings. Auckland, New Zealand: New Zealand Energy Research and Development Committee. 
Barkas, J. (2009, April). Patterns of energy consumption and greenhouse gas emission across primary base metals production. Energy Issues and Climate Change Policies Impacting on the Non-Ferrous Metals Industries. Symposium conducted at the Joint ILZSG_-ICSG_-INSG Energy and Climate Change Policy Seminar, Lisbon, Portugal.

Battelle Columbus Laboratories. (1975). Energy use patterns in metallurgical and nonmetallic mineral processing (phase 4-energy data and flowsheets, high-priority commodities). Washington, DC: Bureau of Mines, U.S. Department of the Interior.

Berge, B. (2001). The ecology of building materials. Oxford, UK: Architecture Press.

Berge, B. (2009). The ecology of building materials, second edition. Oxford, UK: Architecture Press.

Berry, R.S., Long, T.V., and Makino, H. (1977). An international comparison of polymers and their alternatives. In Thomas, J.A.G. (Ed.), Energy analysis (pp. 38-49). Surrey, UK: IPC Science and Technology Press.

Björklund, T., Jönsson, А̊, and Tillman, A-M. (1996). SPINE LCI dataset: ore-based steel production. Gothenburg, Sweden: Chalmers University.

Blanchard, S., and Reppe, P. (1998). Life cycle analysis of a residential home in Michigan. Ann Arbor, MI: Center for Sustainable Systems, University of Michigan.

Boustead, I., and Hancock, G.F. (1979). Handbook of industrial energy analysis. Chichester, UK: Ellis Horwood.

Boustead, I., and Hancock, G.F. (1981). Energy and packaging. Chichester, UK: Ellis Horwood.

Boustead, I. (1993a). SPINE LCI dataset: extraction to polyethylene all grades APME. Brussels, Belgium: Association of Plastic Manufacturers in Europe.

Boustead, I. (1993b). SPINE LCI dataset: extraction to polyethylene. Brussels, Belgium: Association of Plastic Manufacturers in Europe.

Boustead, I. (1993). SPINE LCI dataset: general purpose polystyrene (GPPS). Brussels, Belgium: Association of Plastic Manufacturers in Europe.

Boustead, I. (1993c). SPINE LCI dataset: polypropylene. Brussels, Belgium: Association of Plastic Manufacturers in Europe.

Boustead, I. (1994). SPINE LCI dataset: PVC. Brussels, Belgium: Association of Plastic Manufacturers in Europe.

Boustead, I. (1995). SPINE LCI database: PET. Brussels, Belgium: Association of Plastic Manufacturers in Europe.

Boustead, I. (1996). SPINE LCI dataset: flexible PUR foam. Brussels, Belgium: Association of Plastic Manufacturers in Europe.

Boustead, I. (1996). SPINE LCI dataset: rigid PUR foam. Brussels, Belgium: Association of Plastic Manufacturers in Europe.

Boustead, I. (1997). SPINE LCI dataset: extraction to polycarbonate APME. Brussels, Belgium: Association of Plastic Manufacturers in Europe.

Boustead, I. (1997). SPINE LCI dataset: general purpose polystyrene (GPPS). Brussels, Belgium: Association of Plastic Manufacturers in Europe. 
Boustead, I. (1998). SPINE LCI dataset: copper ore concentrate preparation and delivery. Brussels, Belgium: The International Copper Association.

Boustead, I. (1998). SPINE LCI dataset: production of blister copper. Brussels, Belgium: The International Copper Association

Boustead, I. (1998). SPINE LCI dataset: production of copper anodes. Brussels, Belgium: The International Copper Association

Boustead, I. (1998). SPINE LCI dataset: production of matte copper. Brussels, Belgium: The International Copper Association.

Boustead, I. (1998). SPINE LCI dataset: production of primary copper. Brussels, Belgium: The International Copper Association.

Boustead, I. (1999). SPINE LCI dataset: extraction to ABS APME. Brussels, Belgium: Association of Plastic Manufacturers in Europe.

Boustead, I. (1999). SPINE LCI dataset: extraction to polyethylene HD APME. Brussels, Belgium: Association of Plastic Manufacturers in Europe.

Boustead, I. (1999). SPINE LCI dataset: production of nylon 66 (APME). Brussels, Belgium: Association of Plastic Manufacturers in Europe.

Boustead, I. (1999). SPINE LCI dataset: production of polyethylene resin (HDPE), (APME). Brussels, Belgium: Association of Plastic Manufacturers in Europe.

Boustead, I. (1999). SPINE LCI dataset: production of polyethylene terephthalate (APME). Brussels, Belgium: Association of Plastic Manufacturers in Europe.

Boustead, I. (1999). SPINE LCI dataset: production of polypropylene (APME). Brussels, Belgium: Association of Plastic Manufacturers in Europe.

Boustead, I. (1999). SPINE LCI dataset: production of polystyrene (APME). Brussels, Belgium: Association of Plastic Manufacturers in Europe.

Boustead, I. (1999). SPINE LCI dataset: production of polyvinyl chloride, emulsion polymerised (APME). Brussels, Belgium: Association of Plastic Manufacturers in Europe.

Boustead, I. (1999). SPINE LCI dataset: production of polyvinyl chloride, suspension polymerised (APME). Brussels, Belgium: Association of Plastic Manufacturers in Europe.

Boustead, I. (1999). SPINE LCI dataset: production of PVC calendered sheet (APME). Brussels, Belgium: Association of Plastic Manufacturers in Europe.

Boustead, I. (1999). SPINE LCI dataset: production of PVC injection moulding (APME). Brussels, Belgium: Association of Plastic Manufacturers in Europe.

Boustead, I. (1999). SPINE LCI dataset: production of PVC pipe (APME). Brussels, Belgium: Association of Plastic Manufacturers in Europe.

Boustead, I. (1999). SPINE LCI dataset: production of PVC unplasticised film (APME). Brussels, Belgium: Association of Plastic Manufacturers in Europe.

Boustead, I. (2003). Polymers and energy. In Andrady, A.L. (Ed.), Plastics and the environment (pp. 123-135). Hoboken, NJ: John Wiley and Sons. 
Boustead, I. (2005). Eco-profiles of the European plastics industry. Acrylonitrile-butadiene-styrene copolymer $(A B S)$. Brussels, Belgium: Plastics Europe.

Boustead, I. (2005). Eco-profiles of the European plastics industry. Glass filled Polyamide 66. Brussels, Belgium: Plastics Europe.

Boustead, I. (2005). Eco-profiles of the European plastics industry. High density polyethylene (HDPE). Brussels, Belgium: Plastics Europe.

Boustead, I. (2005). Eco-profiles of the European plastics industry. Liquid epoxy resins. Brussels, Belgium: Plastics Europe.

Boustead, I. (2005). Eco-profiles of the European plastics industry. Polyamide 6 (Nylon 6). Brussels, Belgium: Plastics Europe.

Boustead, I. (2005). Eco-profiles of the European plastics industry. Polyamide 66 (Nylon 66). Brussels, Belgium: Plastics Europe.

Boustead, I. (2005). Eco-profiles of the European plastics industry. Polyethylene terephthalate (PET) (amorphous grade). Brussels, Belgium: Plastics Europe.

Boustead, I. (2005). Eco-profiles of the European plastics industry. Polyethylene terephthalate (PET) (bottle grade). Brussels, Belgium: Plastics Europe.

Boustead, I. (2005). Eco-profiles of the European plastics industry. Polypropylene (PP). Brussels, Belgium: Plastics Europe.

Boustead, I. (2005). Eco-profiles of the European plastics industry. Polyurethane flexible foam. Brussels, Belgium: Plastics Europe.

Boustead, I. (2005). Eco-profiles of the European plastics industry. Polyurethane rigid foam. Brussels, Belgium: Plastics Europe.

Boustead, I. (2005). Eco-profiles of the European plastics industry. Polyvinylchloride (PVC). Brussels, Belgium: Plastics Europe.

Boyd, S.B. (2011). Bio-based versus conventional plastics for electronics housings: LCA literature review. Tempe, AZ: The Sustainability Consortium.

Brandrup, J. (2003). Polymers, polymer recycling, and sustainability. In Andrady, A.L. (Ed.), Plastics and the environment (pp. 523-562). Hoboken, NJ: John Wiley and Sons.

Bridgestone Europe. (2012). Environmental commitment and performance, 2012. Brussels, Belgium: Bridgestone Europe.

Brohammer, G. (1994). SPINE LCI dataset: production of latex rubber. Götenborg, Sweden: Chalmers University of Technology. Retrieved March 15, 2013 from http://cpmdatabase.cpm.chalmers.se/Scripts/sheet.asp?ActId=SCACPM0001998-01-19832

Bruch, H., Gohlke, D., Kruger, C., Reuter, M., Roepenack, I.V., Rombach, E., Rombach, G., and Winkler, P. (1995). LCI of copper production and processing, special printing (s198). Duesseldorf, Germany: German Copper Institute (DKI). 
Bruch, K.H., Gohlke, D., Kögler, C., Kruger, J., Reuter, M., von Röpenack, I., Rombach, E., Rombach, G., and Winkler, P. (1995). SPINE LCI dataset: copper alloy casting of block metal from scrap. Gothenburg, Sweden: Chalmers University of Technology.

Bruch, K.H., Gohlke, D., Kögler, C., Kruger, J., Reuter, M., von Röpenack, I., Rombach, E., Rombach, G., and Winkler, P. (1995). SPINE LCI dataset: copper casting and drawing to 0.06mm wire. Gothenburg, Sweden: Chalmers University of Technology.

Bruch, K.H., Gohlke, D., Kögler, C., Kruger, J., Reuter, M., von Röpenack, I., Rombach, E., Rombach, G., and Winkler, P. (1995). SPINE LCI dataset: copper casting and drawing to 0.6mm wire. Gothenburg, Sweden: Chalmers University of Technology.

Bruch, K.H., Gohlke, D., Kögler, C., Kruger, J., Reuter, M., von Röpenack, I., Rombach, E., Rombach, G., and Winkler, P. (1995). SPINE LCI dataset: copper casting and drawing to 8mm wire. Gothenburg, Sweden: Chalmers University of Technology.

Bruch, K.H., Gohlke, D., Kögler, C., Kruger, J., Reuter, M., von Röpenack, I., Rombach, E., Rombach, G., and Winkler, P. (1995). SPINE LCI dataset: copper casting, drawing and lacquering to 0.6mm wire. Gothenburg, Sweden: Chalmers University of Technology.

Bruch, K.H., Gohlke, D., Kögler, C., Kruger, J., Reuter, M., von Röpenack, I., Rombach, E., Rombach, G., and Winkler, P. (1995). SPINE LCI dataset: copper casting, drawing and polymer coating to 0.6mm wire. Gothenburg, Sweden: Chalmers University of Technology.

Bruch, K.H., Gohlke, D., Kögler, C., Kruger, J., Reuter, M., von Röpenack, I., Rombach, E., Rombach, G., and Winkler, P. (1995). SPINE LCI dataset: copper continuous casting. Gothenburg, Sweden: Chalmers University of Technology.

Bruch, K.H., Gohlke, D., Kögler, C., Kruger, J., Reuter, M., von Röpenack, I., Rombach, E., Rombach, G., and Winkler, P. (1995). SPINE LCI dataset: copper extrusion and drawing to profiles. Gothenburg, Sweden: Chalmers University of Technology.

Bruch, K.H., Gohlke, D., Kögler, C., Kruger, J., Reuter, M., von Röpenack, I., Rombach, E., Rombach, G., and Winkler, P. (1995). SPINE LCI dataset: copper extrusion and drawing to tubes. Gothenburg, Sweden: Chalmers University of Technology.

Bruch, K.H., Gohlke, D., Kögler, C., Kruger, J., Reuter, M., von Röpenack, I., Rombach, E., Rombach, G., and Winkler, P. (1995). SPINE LCI dataset: copper rolling to strips. Gothenburg, Sweden: Chalmers University of Technology.

Bruch, K.H., Gohlke, D., Kögler, C., Kruger, J., Reuter, M., von Röpenack, I., Rombach, E., Rombach, G., and Winkler, P. (1995). SPINE LCI dataset: copper skew rolling, pilgering and drawing to tubes. Gothenburg, Sweden: Chalmers University of Technology.

Bruch, K.H., Gohlke, D., Kögler, C., Kruger, J., Reuter, M., von Röpenack, I., Rombach, E., Rombach, G., and Winkler, P. (1995). SPINE LCI dataset: high purity copper production from primary raw materials. Gothenburg, Sweden: Chalmers University of Technology.

Bruch, K.H., Gohlke, D., Kögler, C., Kruger, J., Reuter, M., von Röpenack, I., Rombach, E., Rombach, G., and Winkler, P. (1995). SPINE LCI dataset: high purity copper production from secondary raw materials. Gothenburg, Sweden: Chalmers University of Technology.

Buchanan, A.H., and Honey, B.G. (1994). Energy and carbon dioxide implications of building construction. Energy and Buildings, 20(3), 205-217. 
Building Research Establishment. (1994). Thermal insulation, avoiding risks. Report $C 1 / S f B(A 3 j)(M 2)$. London, UK: HMSO.

Burnham, A. (2012). Updated vehicle specifications in the GREET vehicle-cycle model. Lemont, IL: Center for Transportation Research, Argonne National Laboratory.

Burnham, A., Wang, M., and Wu, Y. (2006). Development and applications of GREET 2.7—the transportation vehicle-cycle model. Argonne, IL: Argonne National Laboratory.

Centre for Building Performance Research. (2007) Table of embodied energy coefficients. Wellington, New Zealand: Victoria University of Wellington, New Zealand. Retrieved September 12, 2012 from http://www.victoria.ac.nz/cbpr/documents/pdfs/ee-coefficients.pdf

Chapman, P.F. (1977). The energy costs of materials. In Thomas, J.A.G. (Ed.), Energy analysis (pp. 27-37). Surrey, UK: IPC Science and Technology Press.

Chapman, P.F., and Roberts, F. (1983). Metal resources and energy. London, UK: Butterworths.

Chen, Y., Farinelli, U., and Johansson, T.B. (2004). Technological leapfrogging—a strategic pathway to modernisation of the Chinese iron and steel industry? Energy for Sustainable Development, 8(2), 30-38.

Cherubini, F., Raugei, M., and Ulgiati, S. (2008). LCA of magnesium production: technological overview and worldwide estimation of environmental burdens. Resources, Conservation and Recycling, 52(8-9), 1093 1100 .

Citherlet, S. (2001). Towards the holistic assessment of building performance based on an integrated simulation approach. (Doctoral dissertation). Swiss Federal Institute of Technology (EPFL), Lausanne, Switzerland.

Collings, D. (2006). An environmental comparison of bridge forms. Proceedings of the ICE—Bridge Engineering, 159(4), 163-168.

Continental AG. (2013). Environmental goals and key figures. Hannover, Germany: Continental AG. Retrieved April 8, 2013 from http://www.conti-online.com/generator/www/com/en/continental/csr/themes/ ecology/environmental_protection/environmental_goals_key_figures/environmental_goals_key_figures_en .html

Cook, G.D. (1994). Sustainability: viewed both holistically and as a construction application. In Kibert, C.J. (Ed.), Sustainable construction. Proceedings of the first international conference of CIB TG 6, November 6-9, 1994, Tampa, FL (pp. 163-170). Gainesville, FL: University of Florida.

Corbière-Nicollier, T., Laban, B.G., Lundquist, L., Leterrier, Y., Månson, J.-A.E., and Jolliet, O. (2001). Life cycle assessment of biofibres replacing glass fibres as reinforcement in plastics. Resources, Conservation and Recycling, 33(4), 267-287.

Cummings-Sexton, J. (1981). Automobile materials competition: energy implications of fiber-reinforced plastics. Washington, DC: U.S. Department of Energy.

Curlee, T.R., and Das, S. (1998). Viability of recycling plastics by tertiary processes. Solid Waste Technologies, 2(3), 50-57.

Daniel, R.A. (2003). Environmental considerations to structural material selection for a bridge. Paper presented at the European Bridge Engineering Conference, Lightweight Bridge Decks, Rotterdam, the Netherlands, 27 28 March, 2003.

Das, S. (2000). The life-cycle impacts of aluminum body-in-white automotive material. JOM, 52(8), 41-44. 
de Beer, J., Worrell, E., and Blok, K. (1998). Future technologies for energy-efficient iron and steel making. Annual Review of Energy and the Environment, 23(1), 123-205.

DeBenedetti, B., Angelini, E., Grassini, S., Marino, M., Fracassi, F., and Palumbo, F. (2005). Environmental impact and corrosion behaviour assessment of magnesium castings. Materials Science Forum, 488-489, 787-800.

Demkin, J.A. (Ed.) (1996). Environmental resource guide. New York, NY: John Wiley and Sons.

Deng, Y., van Acker, K., Dewulf, W., and Duflou, J.R. (2011). Environmental assessment of printed circuit boards from biobased materials. In Hesselbach, J., and Herrmann, C. (Eds.), Glocalized Solutions for Sustainability in Manufacturing: Proceedings of the $18^{\text {th }}$ CIRP International Conference of Life Cycle Engineering, Technische Universität Braunschweig, Braunschweig, Germany, 2-4 May, 2011 (pp. 605610). Berlin: Springer.

Development Alternatives. (1997). The construction sector in India and climate change. Development Alternatives Newsletter, 7(1). Retrieved March 1, 2013 from http://devalt.org/newsletter/main.htm

Dhingra, R., Overly, J.G., and Davis, G.A. (1999). Life-cycle environmental evaluation of aluminum and composite intensive vehicles. Washington, DC: U.S. Department of Energy.

Dias, W.P.S., and Pooliyadda, S.P. (2004). Quality based energy contents and carbon coefficients for building materials: a systems approach. Energy, 29(4), 561-580.

Eaton, K.J., Gorgolewski, M., Amato, A., and Birtles, T.B. (1999). Using life cycle assessment as a tool for quantifying green buildings. In Conference Proceedings Green Building Challenge '98 (volume 2), October 26-28, 1998, Vancouver, Canada (pp. 237-244). Ottawa, ON: Natural Resources Canada.

Eckelman, M.J., Reck, B.K., and Graedel, T.E. (2012). Exploring the global journey of nickel with Markov Chain models. Journal of Industrial Ecology, 16(3), 334-342.

European Aluminium Association. (2002). SPINE LCI dataset: primary aluminium production. Gothenburg, Sweden: Chalmers University of Technology.

European Aluminium Association. (2002). SPINE LCI dataset: production of extruded aluminium profiles. Gothenburg, Sweden: Chalmers University of Technology.

European Aluminium Association. (2002). SPINE LCI dataset: production of rolled aluminium sheet. Gothenburg, Sweden: Chalmers University of Technology.

European Copper Institute. (2012). The environmental profile of copper products. Brussels, Belgium: European Copper Institute.

Evans, J.W. (2007). The evolution of technology for light metals over the last 50 years: Al, Mg, and Li. JOM, 59(2), 30-38.

Fitch, P.E., and Copper, J.S. (2004). Life cycle energy analysis as a method for material selection. Transactions of the ASME, 126(5), 798-804.

Franklin Associates. (2000). Plastics' energy and greenhouse gas savings using housewrap applied to the exterior of single family residential housing in the U.S. and Canada: a case study. Prairie Village, KS: Franklin Associates.

Franklin Associates. (2001). A life cycle inventory of selected commercial roofing products. Merrickville, ON: Athena Sustainable Materials Institute. 
Franklin Associates. (2006). Life cycle inventory of five products produced from polylactide (PLA) and petroleumbased resins. Prairie Village, KS: Franklin Associates.

Franklin Associates. (2007). LCI summary for PLA and PET 12-ounce water bottles. Prairie Village, KS: Franklin Associates.

Franklin Associates. (2010). Life cycle inventory of 100\% postconsumer HDPE and PET recycled resin from postconsumer containers and packaging. Prairie Village, KS: Franklin Associates.

Franklin Associates. (2011a). Revised final appendices. Cradle-to-gate life cycle inventory of nine plastic resins and four polyurethane precursors. Prairie Village, KS: Franklin Associates.

Franklin Associates. (2011b). Life cycle inventory of plastic fabrication processes: injection molding and thermoforming. Prairie Village, KS: Franklin Associates.

Fthenakis, V.M., Kim, H.C., and Wang, W. (2007). Life cycle inventory analysis in the production of metals used in photovoltaics. Upton, NY: Brookhaven National Laboratory.

Fussler, C.R., and Krummenacher, B. (1991). Ecobalances: a key to better environmental material choices in automobile design. Materials and Design, 12(3), 123-128.

Gaines, L.L. (1981). Energy and materials use in the production and recycling of consumer-goods packaging. Argonne, IL: Argonne National Laboratory, U.S. Department of Energy.

Gielen, D.J. (1998). The future of the European aluminium industry: a MARKAL energy and material flow analysis. In Methodological Aspects of Resource-Oriented Analysis of Material Flows, April 23-24, 1998, Bergisch Gladbach, Germany (pp. 1-14). Bonn, Germany: German Research Association (DFG).

Glass Fibre Europe. 2012). Life cycle assessment of CFGF-continuous filament glass fibre products. Brussels, Belgium: Glass Fibre Europe.

González, M.J., and Navarro, J.G. (2006). Assessment of the decrease of $\mathrm{CO}_{2}$ emissions in the construction field through the selection of materials: practical case study of three houses of low environmental impact. Building and Environment, 41(7), 902-909.

Goodyear. (2011). 2010 corporate responsibility report. Akron, OH: Goodyear.

Goodyear. (2012). 2011 corporate responsibility report. Akron, OH: Goodyear.

Grjotheim, K., Kvande, H., and Zhuxian, Q. (1995). Key improvements to Hall-Héroult since the end of World War II. JOM 47(11), 32-35.

Häkkinen, T., and Mäkelä, K. (1996). Environmental adaption of concrete: environmental impact of concrete and asphalt pavements. Espoo, Finland: Technical Research Centre of Finland.

Hammond, G., and Jones, C. (2008). Inventory of carbon and energy (ICE), version 1.6a. Bath, UK: University of Bath.

Hancock, G.F. (1984). Energy requirements for manufacture of some non-ferrous metals. Metals Technology, 11(7), 290-299.

Hanko, G., Antrekowitsch, H., and Ebner, P. (2002). Recycling automotive magnesium scrap. JOM, 54(2), 51-54.

Hankook Tire. (2011). Hankook Tire CSR report 2010. Seoul, South Korea: Hankook Tire.

Hankook Tire. (2012). Hankook Tire CSR report 2011/12. Seoul, South Korea: Hankook Tire. 
Hansen, K., Krough, H., and Dinesen, J. (1994). Environmental assessment of building projects based upon a life cycle approach. In Kibert, C.J. (Ed.), Sustainable construction. Proceedings of the first international conference of CIB TG 6, November 6-9, Tampa, FL (pp. 203-212). Gainesville, FL: University of Florida.

Hasanbeigi, A., Price, L., Aden, N., Chunxia, Z., Xiuping, L., and Fangqin, S. (2011). A comparison of iron and steel production energy use and energy intensity in China and the U.S. Berkeley, CA: Berkeley National Laboratory, U.S. Department of Energy.

Haupin, W. (1987). Aluminum. In Meyers, R.A. (Ed.), Encyclopaedia of physical science and technology, Volume 1 (pp. 466-491). Orlando, FL: Academic Press.

Haupin, W. (1992). Aluminum. In Meyers, R.A. (Ed.), Encyclopaedia of physical science and technology, Second edition, Volume 1 (pp. 565-590). San Diego, CA: Academic Press.

Hellström, J. (2006). Life cycle assessment of industrial floors. A comparative study of HTC Superfloor ${ }^{\mathrm{TM}}$ and an epoxy floor. Unpublished master's thesis. Linköping University, Linköping, Sweden.

Holtzhausen, H.J. (2007). Embodied energy and its impact on architectural decisions. In Kungolas, A., Brebbia, C.A., and Beriatos, E. (Eds.), Sustainable Development and Planning III, Volume I (pp. 377-385). Southampton, UK: WIT Press.

IEA. (1991a). Energy balances of OECD countries, 1960-1979. Paris: International Energy Agency.

IEA. (1991b). Energy balances of OECD countries, 1980-1989. Paris: International Energy Agency.

IEA. (1992). Energy balances of OECD countries, 1989-1990. Paris: International Energy Agency.

IEA. (1993). Energy balances of OECD countries, 1990-1991. Paris: International Energy Agency.

IEA. (1994). Energy balances of OECD countries, 1991-1992. Paris: International Energy Agency.

IEA. (1995). Energy balances of OECD countries, 1992-1993. Paris: International Energy Agency.

IEA. (1996). Energy balances of OECD countries, 1993-1994. Paris: International Energy Agency.

IEA. (1997). Energy balances of OECD countries, 1994-1995. Paris: International Energy Agency.

IEA. (1998a). Energy balances of OECD countries, 1995-1996. Paris: International Energy Agency.

IEA. (1998b). Energy statistics and balances of non-OECD countries, 1995-1996. Paris: International Energy Agency.

IEA. (1999a). Energy balances of non-OECD countries, 1996-1997. Paris: International Energy Agency.

IEA. (1999b). Energy balances of OECD countries, 1996-1997. Paris: International Energy Agency.

IEA. (2000a). Energy balances of non-OECD countries, 1997-1998. Paris: International Energy Agency.

IEA. (2000b). Energy balances of OECD countries, 1997-1998. Paris: International Energy Agency.

IEA. (2001a). Energy balances of non-OECD countries, 1998-1999. Paris: International Energy Agency.

IEA. (2001b). Energy balances of non-OECD countries, 1998-1999. Paris: International Energy Agency. 
IEA. (2002a). Energy balances of non-OECD countries, 1999-2000. Paris: International Energy Agency.

IEA. (2002b). Energy balances of OECD countries, 1999-2000. Paris: International Energy Agency.

IEA. (2003a). Energy balances of non-OECD countries, 2000-2001. Paris: International Energy Agency.

IEA. (2003b). Energy balances of OECD countries, 2000-2001. Paris: International Energy Agency.

IEA. (2004a). Energy balances of non-OECD countries, 2001-2002. Paris: International Energy Agency.

IEA. (2004b). Energy balances of OECD countries, 2001-2002. Paris: International Energy Agency.

IEA. (2005a). Energy balances of non-OECD countries, 2002-2003. Paris: International Energy Agency.

IEA. (2005b). Energy balances of OECD countries, 2002-2003. Paris: International Energy Agency.

IEA. (2006a). Energy balances of non-OECD countries, 2003-2004. Paris: International Energy Agency.

IEA. (2006b). Energy balances of OECD countries, 2003-2004. Paris: International Energy Agency.

IEA. (2007a). Energy balances of non-OECD countries, 2004-2005. Paris: International Energy Agency.

IEA. (2007b). Energy balances of OECD countries, 2004-2005. Paris: International Energy Agency.

IEA. (2008a). Energy balances of non-OECD countries, 2008 edition. Paris: International Energy Agency.

IEA. (2008b). Energy balances of OECD countries, 2008 edition. Paris: International Energy Agency.

IEA. (2009a). Energy balances of non-OECD countries, 2009 edition. Paris: International Energy Agency.

IEA. (2009b). Energy balances of OECD countries, 2009 edition. Paris: International Energy Agency.

IEA. (2010a). Energy balances of non-OECD countries, 2010 edition. Paris: International Energy Agency.

IEA. (2010b). Energy balances of OECD countries, 2010 edition. Paris: International Energy Agency.

IEA. (2011a). Energy balances of non-OECD countries, 2011 edition. Paris: International Energy Agency.

IEA. (2011b). Energy balances of non-OECD countries, 2011 edition. Paris: International Energy Agency.

IEA. (2012a). Energy balances of non-OECD countries, 2012 edition. Paris: International Energy Agency.

IEA. (2012b). Energy balances of OECD countries, 2012 edition. Paris: International Energy Agency.

Institut Bauen und Umwelt e.V. (2010). Environmental product declaration. Structural steel: sections and plates. bauforumstahl. Königswinter, Germany: Institut Bauen und Umwelt e.V.

Institut Bauen und Umwelt e.V. (2010). Environmental product declaration. TECUß-copper strips and copper alloys. KME Germany GmbH. Königswinter, Germany: Institut Bauen und Umwelt e.V.

Institut Bauen und Umwelt e.V. (2011). Environmental product declaration. Rolled lead coils and rolled lead sheets. Röhr + Stolberg GMBH. Königswinter, Germany: Institut Bauen und Umwelt e.V.

Institut Bauen und Umwelt e.V. (2011). Environmental product declaration. Structural section steel. CELSA Barcelona. Königswinter, Germany: Institut Bauen und Umwelt e.V. 
Institut Bauen und Umwelt e.V. (2012). Environmental product declaration. Light gauge steel profiles. Akkon Steel structure Systems Co. Königswinter, Germany: Institut Bauen und Umwelt e.V.

Institut Bauen und Umwelt e.V. (2012). Environmental product declaration. TECU® copper alloys sheets and strips (TECUß Bronze, TECUß Gold). KME Germany GmbH. Königswinter, Germany: Institut Bauen und Umwelt e.V.

Institut Bauen und Umwelt e.V. (2012). Environmental product declaration. TECU®-Eco copper sheets and strips (TECU® Classic Eco, TECU® Oxid Eco, TECU® Patina Eco). KME Germany GmbH. Königswinter, Germany: Institut Bauen und Umwelt e.V.

International Aluminium Institute. (2007). Life cycle assessment of aluminium: inventory data for the primary aluminium industry. Year 2005 update. London, UK: International Aluminium Institute.

International Aluminium Institute. (2012). Primary aluminium smelting energy intensity. London, UK: International Aluminium Institute. Retrieved December 25, 2012, from http://www.world-aluminium.org/statistics/ primary-aluminium-smelting-energy-intensity/\#data

International Iron and Steel Institute. (1978). A handbook of world steel statistics. Brussels, Belgium: International Iron and Steel Institute.

International Iron and Steel Institute. (1980). Steel statistical yearbook 1980. Brussels, Belgium: International Iron and Steel Institute.

International Iron and Steel Institute. (1981). Steel statistical yearbook 1981. Brussels, Belgium: International Iron and Steel Institute.

International Iron and Steel Institute. (1982). Steel statistical yearbook 1982. Brussels, Belgium: International Iron and Steel Institute.

International Iron and Steel Institute. (1983). Steel statistical yearbook 1983. Brussels, Belgium: International Iron and Steel Institute.

International Iron and Steel Institute. (1984). Steel statistical yearbook 1984. Brussels, Belgium: International Iron and Steel Institute.

International Iron and Steel Institute. (1985). Steel statistical yearbook 1985. Brussels, Belgium: International Iron and Steel Institute.

International Iron and Steel Institute. (1986). Steel statistical yearbook 1986. Brussels, Belgium: International Iron and Steel Institute.

International Iron and Steel Institute. (1987). Steel statistical yearbook 1987. Brussels, Belgium: International Iron and Steel Institute.

International Iron and Steel Institute. (1988). Steel statistical yearbook 1988. Brussels, Belgium: International Iron and Steel Institute.

International Iron and Steel Institute. (1989). Steel statistical yearbook 1989. Brussels, Belgium: International Iron and Steel Institute.

International Iron and Steel Institute. (1990). Steel statistical yearbook 1990. Brussels, Belgium: International Iron and Steel Institute. 
International Iron and Steel Institute. (1991). Steel statistical yearbook 1991. Brussels, Belgium: International Iron and Steel Institute.

International Iron and Steel Institute. (1992). Steel statistical yearbook 1992. Brussels, Belgium: International Iron and Steel Institute.

International Iron and Steel Institute. (1993). Steel statistical yearbook 1993. Brussels, Belgium: International Iron and Steel Institute.

International Iron and Steel Institute. (1994). Steel statistical yearbook 1994. Brussels, Belgium: International Iron and Steel Institute.

International Iron and Steel Institute. (1995). Steel statistical yearbook 1995. Brussels, Belgium: International Iron and Steel Institute.

International Iron and Steel Institute. (1996). Steel statistical yearbook 1996. Brussels, Belgium: International Iron and Steel Institute.

International Iron and Steel Institute. (1997). Steel statistical yearbook 1997. Brussels, Belgium: International Iron and Steel Institute.

International Iron and Steel Institute. (1998). Steel statistical yearbook 1998. Brussels, Belgium: International Iron and Steel Institute.

International Iron and Steel Institute. (1999). Steel statistical yearbook 1999. Brussels, Belgium: International Iron and Steel Institute.

International Iron and Steel Institute. (2000). Steel statistical yearbook 2000. Brussels, Belgium: International Iron and Steel Institute.

International Iron and Steel Institute. (2001). Steel statistical yearbook 2001. Brussels, Belgium: International Iron and Steel Institute.

International Iron and Steel Institute. (2002). Steel statistical yearbook 2002. Brussels, Belgium: International Iron and Steel Institute.

International Iron and Steel Institute. (2003). Steel statistical yearbook 2003. Brussels, Belgium: International Iron and Steel Institute.

International Iron and Steel Institute. (2004a). The measure of our sustainability. Report of the world steel industry 2004. Brussels, Belgium: International Iron and Steel Institute.

International Iron and Steel Institute. (2004b). Steel statistical yearbook 2004. Brussels, Belgium: International Iron and Steel Institute.

International Iron and Steel Institute. (2005). Steel statistical yearbook 2003. Brussels, Belgium: International Iron and Steel Institute.

International Iron and Steel Institute. (2006). Steel statistical yearbook 2006. Brussels, Belgium: International Iron and Steel Institute.

International Iron and Steel Institute. (2007). Steel statistical yearbook 2007. Brussels, Belgium: International Iron and Steel Institute.

International Stainless Steel Institute. (2010). World stainless steel LCI. Brussels, Belgium: International Stainless Steel Institute. 
Karakoussis, V., Leach, M., van der Vorst, R., Hart, D., Lane, J., Pearson, P., and Kilner, J. (2000). Environmental emissions of SOFC and SPFC system manufacture and disposal. London, UK: Imperial College of Science, Technology and Medicine.

Kellogg, H.H. (1989). Energy for metal production in the $21^{\text {st }}$ century. In Koch, M., and Taylor, J. (Eds.), Production and technology in the metallurgical industries (pp. 145-155). Warrendale, PA: The Minerals, Metals and Materials Society.

Kemmoku, Y., Ishikawa, K., Nakagawa, S., Kawamoto, T., and Sakakibara, T. (2002). Life cycle $\mathrm{CO}_{2}$ emissions of a photovoltaic/wind/diesel generating system. Electrical Engineering in Japan, 138(2), 14-23.

Keoleian, G., Miller, S., Kleine, R.D., Fang, A., and Mosley, J. (2012). Life cycle material data update for GREET model. Ann Arbor, MI: University of Michigan.

Khan, F.I., Hawboldt, K., and Iqbal, M.T. (2005). Life cycle analysis of wind-fuel cell integrated system. Renewable Energy, 30(2), 157-177.

Kiani, M.R. (2005). Evaluate the whole life environmental impact of glass in a fully glazed commercial building. In The 2005 World Sustainable Building Conference, Tokyo, Japan, September 27-29, 2005 (pp. 1163-1170). Tokyo, Japan: Institute of International Harmonization for Building and Housing (IIBH).

Kindler, H., and Nikles, A. (1979). Energieaufwand zur Herstellung von Werkstoffen—Berechnungsgrundsätze und Energieäquivalenzwerte von Kunststoffen. Kunststoffe, 70, 802-807.

Knight, L., Huff, M., Stockhausen, J.I., and Ross, R.J. (2005). Comparing energy use and environmental emissions of reinforced wood doors and steel doors. Forest Products Journal, 55(6), 48-52.

Krömer, S., Kreipe, E., Reichenbach, D., and Stark, R. (1999). Life cycle assessment of a car tire. Hannover, Germany: Continental AG.

La Rosa, A., Cozzo, G., Latteri, A., Mancini, G., Recca, A., and Cicala, G. (2013). A comparative life cycle assessment of a composite component for automotive. Chemical Engineering Transactions, 32, 1723-1728.

Lange, W. (2003). Polymers in automobile applications. In Andrady, A.L. (Ed.), Plastics and the environment (pp. 727-746). Hoboken, NJ: John Wiley and Sons.

Langley, K.F. (1986). Energy efficiency in the UK iron and steel industry. Applied Energy, 23(2), 73-107.

Lawson, B. (1996). Building materials energy and the environment. Towards ecologically sustainable development. Barton, Australia: The Royal Australian Institute of Architects.

Li, N. (2004, December). Automotive magnesium applications and life cycle environmental assessment. $3^{\text {rd }}$ International Conference on SF6 and the Environment. Lecture conducted from Scottsdale, AZ.

Li, S., Li, N., Li, J., and Gao, Y. (2012). Vehicle cycle energy and carbon dioxide analysis of passenger car in China. AASRI Procedia, 2, 25-30.

Malcolm Pirnie. (2007). Greenhouse gas inventory and product life cycle analysis_phase II. Tucker, GA: Lehigh Technologies.

Mayyas, A.T., Qattawi, A., Mayyas, A.R., and Omar, M. (2012). Life cycle assessment-based selection for a sustainable lightweight body-in-white design. Energy, 39(1), 412-425. 
Michelin. (2010). 2009 annual and sustainable development report. Clermont-Ferrand, France: Michelin.

Michelin. (2011). 2010 annual and sustainable development report. Clermont-Ferrand, France: Michelin.

Michelin. (2012). 2011 annual and sustainable development report. Clermont-Ferrand, France: Michelin.

Michelin. (2013). 2012 corporate governance and results. Clermont-Ferrand, France: Michelin.

Najdenov, I., Raić, K.T., and Kokeza, G. (2012). Aspects of energy reduction by autogenous copper production in the copper smelting plant Bor. Energy, 43(1), 376-384.

Nickel Institute. (2000). Life cycle assessment of nickel products. Toronto, ON: Nickel Institute.

Nicolaos, D., and Antonios, Z. (1993). Saving energy in alumina-aluminum plants. In Pilavachi, P.A. (Ed.), Energy efficiency in process technology (pp. 73-88). London, UK: Elsevier Applied Science.

Nirula, S.C. (1991). Acrylonitrile (Report 17b, Supplement B): a private report by the Process Economics Program. Menlo Park, CA: SRI International.

Nokian Tyres. (2004). Environmental report 2003. Nokia, Finland: Nokian Tyres.

Nokian Tyres. (2006). EMAS report 2005. Nokia, Finland: Nokian Tyres.

Nokian Tyres. (2008). EMAS report 2007. Nokia, Finland: Nokian Tyres.

Nokian Tyres. (2009). EMAS report 2008. Nokia, Finland: Nokian Tyres.

Nokian Tyres. (2010). Environmental report 2009. Nokia, Finland: Nokian Tyres.

Nokian Tyres. (2011). EMAS report 2010. Nokia, Finland: Nokian Tyres.

Nokian Tyres. (2012). EMAS report 2011. Nokia, Finland: Nokian Tyres.

Norgate, T.E., and Rankin, W.J. (2000). Life cycle assessment of copper and nickel production. In Proceedings, MINPREX 2000, International Conference on Minerals Processing and Extractive metallurgy, Melbourne, Australia, September 11-13, 2000 (pp. 133-138). Carlton, Australia: Australasian Institute of Mining and Metallurgy.

Norgate, T. E., and Rankin, W. J. (2002). An environmental assessment of lead and zinc production processes. In Proceedings, Green Processing 2002, International Conference on the Sustainable Processing of Minerals, Cairns, Australia, May 29-31, 2002 (pp. 177-184). Carlton, Australia: Australasian Institute of Mining and Metallurgy.

Norgate, T. E., and Rankin, W. J. (2002). The role of metals in sustainable development. In Proceedings, Green Processing 2002, International Conference on the Sustainable Processing of Minerals, Cairns, Australia, May 29-31, 2002 (pp. 49-55). Carlton, Australia: Australasian Institute of Mining and Metallurgy.

Norgate, T.E., Jahanshahi, S., and Rankin, W.J. (2004). Alternative routes to stainless steel-a life cycle approach. In Tenth International Ferroalloys Congress, Cape Town, South Africa, February 1-4, 2004 (pp. 693-704). Randburg, South Africa: International Ferro-Alloys Congress (INFACON).

Norgate, T.E., Jahanshahi, S., and Rankin, W.J. (2007). Assessing the environmental impact of metal production processes. Journal of Cleaner Production, 15(8-9), 838-848. 
Nyboer, J., and Jaccard, I. (2010). A review of energy consumption and related data: Canadian iron and steel and ferro-alloy manufacturing industries 1990 to 2008. Burnaby, BC: Canadian Industrial Energy End-use Analysis Centre, Simon Fraser University.

Nyboer, J., and Kniewasser, M. (2012a). Development of energy intensity indicators for Canadian industry, 1990 to 2010. Burnaby, BC: Canadian Industrial Energy End-use Analysis Centre, Simon Fraser University.

Nyboer, J., and Kniewasser, M. (2012b). Energy use and related data: Canadian aluminium industries 1990 to 2010. Burnaby, BC: Canadian Industrial Energy End-use Analysis Centre, Simon Fraser University.

Nyboer, J., and Moulé, D. (2012). Energy use and related data: Canadian foundry industry 1990 to 2010. Burnaby, BC: Canadian Industrial Energy End-use Analysis Centre, Simon Fraser University.

Nyboer, J., and Rudd, S. (2011). A review of energy consumption and related data: Canadian mining and smelting and refining industries 1990 to 2009. Burnaby, BC: Canadian Industrial Energy End-use Analysis Centre, Simon Fraser University.

Olsson, A. (2002). SPINE LCI database: production of EPDM. Gothenburg, Sweden: Swedish Environmental Research Institute.

Ostermayer, A., and Giegrich, J. (2006a). Eco-profiles of the European Plastics Industry. Polyvinylchloride (PVC) (emulsion polymerisation). Brussels, Belgium: The European Council of Vinyl Manufacturers and Plastics Europe.

Ostermayer, A., and Giegrich, J. (2006b). Eco-profiles of the European Plastics Industry. Polyvinylchloride (PVC) (suspension polymerisation). Brussels, Belgium: The European Council of Vinyl Manufacturers and Plastics Europe.

Papassava, S., Kia, s., Claya, J., and Gunther, R. (2000). Life cycle analysis of automotive powder primers. In Proceedings of the $27^{\text {th }}$ international waterborne higher solids and powder coatings symposium, New Orleans, LA, March 1-3, 2000 (pp. 383-393). Hattiesburg, MS, University of Southern Mississippi.

Papasavva, S., Kia, S., Claya, J., and Gunther, R. (2001). Characterization of automotive paints: an environmental impact analysis. Progress in Organic Coatings, 43(1), 193-206.

Patel, M. (1999). KEA für produkte der organischen chemie. Karlsruhe, Germany: Fraunhofer-Institut für Systemtechnik und Innovationsforschung (FhG-ISI).

Patel, M., and Mutha, N. (2004). Plastics production and energy. In Cleveland, C.J. (Ed.), Encyclopedia of energy, volume 5 (pp. 81-91). San Diego, CA: Academic Press.

PE Americas. (2010). Environmental overview: complete life cycle assessment of North American container glass. Alexandria, VA: The Glass Packaging Institute (GPI).

Pilz, H., Schweighofer, J., and Kletzer, E. (2005). The contribution of plastic products to resource efficiency. Vienna, Austria: GUA Gessellschaft für umfassende Analysen.

Pirelli. (2000). Environmental report 2000. Sustainability abstract. Milan, Italy: Pirelli.

Pirelli. (2001). Environmental report 2001. Sustainability abstract. Milan, Italy: Pirelli.

Pirelli. (2002). Environmental report 2002. Sustainability abstract. Milan, Italy: Pirelli.

Pirelli. (2003). Environmental report 2003. Sustainability abstract. Milan, Italy: Pirelli.

Pirelli. (2004). Environmental report 2004. Sustainability abstract. Milan, Italy: Pirelli. 
Pirelli. (2005). Annual report 2005. Sustainability abstract. Milan, Italy: Pirelli.

Pirelli. (2006). Annual report 2006. Sustainability abstract. Milan, Italy: Pirelli.

Pirelli. (2007). Sustainability report. Milan, Italy: Pirelli.

Pirelli. (2008). Sustainability report. Milan, Italy: Pirelli.

Pirelli. (2009). Sustainability report at December 31, 2009. Milan, Italy: Pirelli.

Pirelli. (2010). Sustainability report 2010. Milan, Italy: Pirelli.

Pirelli. (2011). Sustainability report 2011. Milan, Italy: Pirelli.

Plastics Europe. (2005). Process data set: polyethylene terephthalate (PET) granulate; production mix, at plant; amorphous. Brussels, Belgium: Plastics Europe.

Plastics Europe. (2005). Process data set: polyethylene terephthalate (PET) granulate; production mix, at plant; bottle grade. Brussels, Belgium: Plastics Europe.

Plastics Europe. (2011). Eco-profiles and environmental product declarations of the European Plastics Manufacturers. Polycarbonate (PC). Brussels, Belgium: Plastics Europe.

Plastics Europe. (2012). Eco-profiles and environmental product declarations of the European Plastics Manufacturers. General-purpose polystyrene (GPPS) and high-impact polystyrene (HIPS). Brussels, Belgium: Plastics Europe.

Potting, J., and Blok, K. (1995). Life-cycle assessment of four types of floor covering. Journal of Cleaner Production, 3(4), 201-213.

Price, L., Worrell, E., Martin, N., Lehman, B., and Sinton, J. (2000). China's industrial sector in an international context. Berkeley, CA: Berkeley National Laboratory, U.S. Department of Energy.

Ramakrishnan, S., and Kotun, P. (2004). Global warming impact of the magnesium produced in China using the Pidgeon process. Resources, Conservation and Recycling, 42(1), 49-64.

Rautaruukki Corporation. (2011). Environmental product declaration and safety information sheet. Colour coated steel plates, sheets and coils. Helsinki, Finland: Rautaruukki Corporation.

Rautaruukki Corporation. (2011). Environmental product declaration and safety information sheet. Hot-rolled steel plates, sheets and coils. Helsinki, Finland: Rautaruukki Corporation.

Reddy, B.V.V., and Jagadish, K.S. (35). Embodied energy of common and alternative building materials and technologies. Energy and Buildings, 35(2), 129-137.

RTI International. (2003). Life-cycle inventory data sets for material production of aluminum, glass, paper, plastic, and steel in north America. Research Triangle Park, NC: RTI International.

Rydh, C.J., and Sun, M. (2005). Life cycle inventory data for materials grouped according to environmental and material properties, Journal of Cleaner Production, 13(13-14), 1258-1268.

Saito, M., and Shukuya, M. (1996). Energy and material use in the production of insulating glass windows. Solar Energy, 58(4-6), 247-252. 
Sandberg, H., Lagneborg, R., Lindblad, B., Axelsson, H., and Bentell, L. (2001). $\mathrm{CO}_{2}$ emissions of the Swedish steel industry. Scandinavian Journal of Metallurgy, 30(6), 420-425.

Scheuer, C., Keoleian, G.A., and Reppe, P. (2003). Life cycle energy and environmental performance of a new university building: modeling challenges and design implications. Energy and Buildings, 35(10), 10491064.

Schexnayder, S.M., Das, S., Dhingra, R., Overly, J.G., Tonn, B.E., Peretz, J.H., Waidley, G., and Davis, G.A. (2001). Environmental evaluation of new generation vehicles and vehicle components. Washington, DC: U.S. Department of Energy.

Schleisner, L. (2000). Life cycle assessment of a wind farm and related externalities. Renewable Energy, 20(3), 279288.

Schuckert, M. (1996). Life cycle analysis: getting the total picture on vehicle engineering alternatives. Automotive Engineering, 104(3), 49-52.

Schüller, M., Estrada, A., and Bringezu, S. (2008). Mapping environmental performance of international material production flows: a comparative case study for the copper industry of Chile and Germany. Minerals and Energy, 23(1), 29-45.

Schwarz, H.-G., Erlangen, B., Krüger, B., and Kuchshinrichs, J.W. (2000). Studies on the global material flow of primary aluminium. Aluminium, 76(1/2), 6-64.

Schwarz, H.-G. (2004). Aluminium production and energy. In Cleveland, C.J. (Ed.), Encyclopedia of energy, volume 1 (pp. 81-95). San Diego, CA: Academic Press.

Selke, S.E.M. (2003). Plastics in packaging. In Andrady, A.L. (Ed.), Plastics and the environment (pp. 139-183). Hoboken, NJ: John Wiley and Sons.

Seppälä, J., Koskela, S., Melanen, M., and Palperi, M. (2002). The Finnish metals industry and the environment. Resources, Conservation and Recycling, 35(1), 61-76.

Sinton, C.W. (2004). Glass and energy. In Cleveland, C.J. (Ed.), Encyclopedia of energy, volume 3 (pp. 1-10). San Diego, CA: Academic Press.

Spriensma, R., Alvarado, C., and Goedkoop, M. (2001). Life cycle assessment of an average European car tyre. Amersfoort, the Netherlands: PRé Consultants.

Stammer, R.E., and Stodolsky, F. (1995). Assessment of the energy impacts of improving highway-infrastructure materials. Argonne, IL: Argonne National Laboratory.

Stobart, P.D. (1986). Centenary of the Hall and Héroult processes 1886-1986. London, UK: International Primary Aluminium Institute.

Stodolsky, F., Vyas, A., Cuenca, R., and Gaines, L. (1995). Life-cycle energy savings potential from aluminumintensive vehicles. In Proceedings, SAE Total Life Cycle Conference and Exposition, Vienna, Austria, October 16-19, 1995. Warrendale, PA: SAE International.

Stripple, H. (2001). Life cycle assessment of road: a pilot study for inventory analysis. Gothenburg, Sweden: Swedish Environmental Research Institute.

Stubbles, J. (2000). Energy use in the U.S. steel industry: an historical perspective and future opportunities. Washington, DC: U.S. Department of Energy. 
Sullivan, J., and Hu, J. (1995). Life cycle energy analysis for automobiles. SAE Technical Paper 951829. Warrendale, PA: Society of Automotive Engineers.

Sun, W., Cai., J., and Ye, Z. (2013). Advances in energy conservation of the China steel industry. The Scientific World Journal, 2013, 1-8. doi:10.1155/2013/247035

Suzuki, T., and Takahashin, J. (2005). Prediction of energy intensity of carbon fiber reinforced plastics for massproduced passenger cars. In Proceedings of the Ninth Japan International SAMPE Symposium, Tokyo, Japan, 29 November-2 December, 2005 (pp. 14-19). Kamakura, Japan: Society for the Advancement of Material and Process Engineering (SAMPE) Japan.

Suzuki, T., Odai, T., Hukui, R., and Takahashi, J. (2009). LCA of passenger vehicles lightened by recyclable carbon fiber reinforced plastics. Paper presented at the International Conference on Life Cycle Assessment, San Jose, Costa Rica, April 25-28.

Tanaka, K. (2008). Assessment of energy efficiency performance measures in industry and their application for policy. Energy Policy, 36(8), 2887-2902.

Teck Cominco. (2005). The value of sustainability. Sustainability report 2005. Vancouver, BC: Teck Cominco.

Teck Cominco. (2006a). Highland Valley copper. Operation and site performance 2006. Vancouver, BC: Teck Cominco.

Teck Cominco. (2006b). Train Operations. Operations and site performance 2006. Vancouver, BC: Teck Cominco.

Teck Cominco. (2007). 2007 sustainability summary. Highland Valley copper operations. Vancouver, BC: Teck Cominco.

Teck Cominco. (2008). 2008 sustainability summary. Carmen de Andacollo. Andacollo, Chile: Teck Cominco.

Teck Cominco. (2008). 2008 sustainability summary. Duck Pond operations. Millertown, NL: Teck Cominco.

Teck Cominco. (2008). 2008 sustainability summary. Quebrada Blanca. Iquique, Chile: Teck Cominco.

Tempelman, E. (2011). Multi-parametric study of the effect of materials substitution on life cycle energy use and waste generation of passenger car structures. Transportation Research Part D, 16(7), 479-485.

Thiriez, A., and Gutowski, T. (2006). An environmental analysis of injection molding. In Proceedings of the 2006 IEEE international symposium on electronics and the environment, Scottsdale, AZ, May 8-11, 2006 (pp. 195-200). Los Alamitos, CA: IEEE Computer Society.

Thomas, J.A.G. (Ed.) (1977). Energy analysis. Surrey, UK: IPC Science and Technology Press.

Thormark, C. (2001). Conservation of energy and natural resources by recycling building waste. Resources, Conservation and Recycling, 33(2), 113-130.

Tikana, L., Sievers, H., and Klassert, A. (2005). Life cycle assessment of copper products. Düsseldorf, Germany: Deutsches Kupferinstitut—Life Cycle Centre.

Tillman, A-M. (1991). SPINE LCI dataset: glassworks. Götenborg, Sweden: Chalmers University of Technology. Retrieved March 15, 2013 from http://cpmdatabase.cpm.chalmers.se/Scripts/sheet.asp?ActId=cpmcth321$6 / 10 / 98-839$

US Environmental Protection Agency. (2003). Background document for life-cycle greenhouse gas emission factors for carpet and personal computers. Atlanta, GA: US Environmental Protection Agency. 
US Environmental Protection Agency. (2013). Waste reduction model (WARM). Atlanta, GA: US Environmental Protection Agency.

Usbeck, V.C., Pflieger, J., and Sun, T. (2011). Life cycle assessment of float glass. Leinfelden-Echterdingen, Germany: PE International.

Voorspools, K.R., Brouwers, E.A., and D'haeseleer, W.D. (2000). Energy content and indirect greenhouse gas emissions embedded in 'emission-free' power plants: results for the Low Countries. Applied Energy, 67(3), 307-330.

Wang, M., Burnham, A., and Wu, Y. (2012). GREET 2 Model. Argonne, IL: Argonne National Laboratory.

Wang, Y., Zhang, Y., Polk, M.B., Kumar, S., and Muzzy, J.D. (2003). Recycling of carpet and textile fibers. In Andrady, A.L. (Ed.), Plastics and the environment (pp. 77-121). Hoboken, NJ: John Wiley and Sons.

Williams, E.D., Weber, C.L., and Hawkins, T.R. (2009). Hybrid framework for managing uncertainty in life cycle inventories. Journal of Industrial Ecology, 13(6), 928-944.

Woolley, T., Kimmins, S., Harrison, P., and Harrison, R. (2001). Green building handbook. London, UK: Spon Press (Taylor and Francis).

World Steel Association. (2008). Steel statistical yearbook 2008. Brussels, Belgium: World Steel Association.

World Steel Association. (2009). Steel statistical yearbook 2009. Brussels, Belgium: World Steel Association.

World Steel Association. (2010). Steel statistical yearbook 2010. Brussels, Belgium: World Steel Association.

World Steel Association. (2011a). LCI data for steel products. Brussels, Belgium: World Steel Association.

World Steel Association. (2011b). Life cycle assessment methodology report. Brussels, Belgium: World Steel Association.

World Steel Association. (2011c). Steel statistical yearbook 2011. Brussels, Belgium: World Steel Association.

Worrell, E., de Beer, J., and Blok, K. (1993). Energy conservation in the iron and steel industry. In Pilavachi, P.A. (Ed.), Energy efficiency in process technology (pp. 91-100). London, UK: Elsevier Applied Science.

Worrell, E., van Heijningen, R.J.J., de Castro, J.F.M., Hazewinkel, J.H.O., de Beer, J.G., Faaij, A.P.C., and Vringer, K. (1994a). New gross energy-requirement figures for materials production. Energy, 19(6), 627-640.

Worrell, E., Cuelenaere, R.F.A., Blok, K., and Turkenburg, W.C. (1994b). Energy consumption by industrial processes in the European Union. Energy, 19(11), 1113-1129.

Worrell, E., de Beer, J., Faaij, A.P.C., and Blok, K. (1994c). Potential energy savings in the production route for plastics. Energy Conservation and Management, 35(12), 1073-1085.

Worrell, E. (1995). Advanced technologies and energy efficiency in the iron and steel industry in China. Energy for Sustainable Development, 11(4), 27-40.

Worrell, E., Price, L., Martin, N., Farla, J., and Schaeffer, R. (1997). Energy intensity in the iron and steel industry: a comparison of physical and economic indicators. Energy Policy, 25(7-9), 727-744.

Worrell, E., Price, L., and Martin, N. (2001). Energy efficiency and carbon dioxide emissions reduction opportunities in the US iron and steel sector. Energy, 26(5), 513-536. 
Worrell, E., Neelis, M., Price, L., Galitsky, C., and Nan, Z. (2007). World best practice energy intensity values for selected industrial sectors. Berkeley, CA: Lawrence Berkeley National Laboratory.

Worrell, E., Price, L., Neelis, M., Galitsky, C., and Nan, Z. (2008). World best practice energy intensity values for selected industrial sectors. Berkeley, CA: Lawrence Berkeley National Laboratory.

Wötzel, K., Wirth, R., and Flake, M. (1999). Life cycle studies on hemp fibre reinforced components and ABS for automotive parts. Die Angewandte Makromolekulare Chemie, 272(4763), 121-127.

Xstrata Copper Canada. (2008). Sustainability report 2008. Toronto, ON: Xstrata Copper Canada.

Xstrata Copper Canada. (2009). Sustainability report 2009. Toronto, ON: Xstrata Copper Canada.

Xstrata Copper Canada. (2010). Sustainability report 2010. Toronto, ON: Xstrata Copper Canada.

Xstrata Copper Canada. (2011). Sustainability report 2011. Toronto, ON: Xstrata Copper Canada.

Xstrata Copper Canada. (2012). Sustainability report 2012. Toronto, ON: Xstrata Copper Canada.

Xstrata Copper Chile. (2011). Altonorte metallurgical facility. 2011 sustainability report. Antofagasta, Chile: Xstrata Copper Chile.

Xstrata Copper Chile. (2011). Xstrata Lomas Bayas. 2011 sustainability report. Santiago, Chile: Xstrata Copper.

Yokohama Tire Philippines. (2012). 2011 sustainability report. Clark Freeport Zone, Philippines: Yokohama Tire Philippines.

Zapata, P., and Gambatese, J.A. (2005). Energy consumption of asphalt and reinforced concrete pavement materials and construction. Journal of Infrastructure Systems, 11(1), 9-20. 
APPENDIX C-COMPLETE LIST OF COUNTRIES IN FIGURES 3.12 AND 3.13

\begin{tabular}{|c|c|c|}
\hline Temporality & Spaciality & \\
\hline Australia & North & Canada \\
\hline Austria & America & Mexico \\
\hline Belgium & & United States \\
\hline Brazil & & \\
\hline Canada & Oceania & Australia \\
\hline Chile & & New Zealand \\
\hline China & & \\
\hline Czech Republic & \begin{tabular}{|l} 
Europe \\
\end{tabular} & Austria \\
\hline Denmark & & Belgium \\
\hline Estonia & & Czech Republic \\
\hline Finland & & Denmark \\
\hline France & & Estonia \\
\hline Germany & & Finland \\
\hline Greece & & France \\
\hline Hungary & & Germany \\
\hline India & & Greece \\
\hline Ireland & & Hungary \\
\hline Israel & & Ireland \\
\hline Italy & & Italy \\
\hline Japan & & Latvia \\
\hline Latvia & & Lithuania \\
\hline Lithuania & & Luxembourg \\
\hline Luxembourg & & Netherlands \\
\hline Mexico & & Norway \\
\hline Netherlands & & Poland \\
\hline New Zealand & & Portugal \\
\hline Norway & & Slovak Republic \\
\hline Philippines & & Slovenia \\
\hline Poland & & Spain \\
\hline Portugal & & Sweden \\
\hline Serbia & & Switzerland \\
\hline Slovak Republic & & Turkey \\
\hline Slovenia & & Ukraine \\
\hline South Africa & & United Kingdom \\
\hline South Korea & & \\
\hline Spain & & \\
\hline Sri Lanka & & \\
\hline Sweden & & \\
\hline Switzerland & & \\
\hline Turkey & & \\
\hline Ukraine & & \\
\hline United Kingdom & & \\
\hline United States & & \\
\hline
\end{tabular}




\section{REFERENCES}

Ayer, N.W., Tyedmers, P.H., Pelletier, N.L., Sonesson, U., and Scholz, A. (2007). Co-product allocation in life cycle assessments of seafood production systems: review of problems and strategies. International Journal of Life Cycle Assessment, 12(7), 480-487.

Baker, J.W., and Lepech, M.D. (2009). Treatment of uncertainties in life cycle assessment. In Proceedings of the 10th International Conference on Structural Safety and Reliability, Osaka, Japan, September 13-17, 2009. New York, NY: International Association for Structural Safety and Reliability International (IASSAR).

Bárzaga-Castellanos, L., Neufert, R., Kayser, G., and Markert, B. (1999). Life cycle assessment of the selective catalytic reduction process for power plants. International Journal of Life Cycle Assessment, 4(6), 329-338.

Berry, R.S., and Fels, M.F. (1972). The production and consumption of automobiles. An energy analysis of the manufacture, discard and reuse of the automobile and its component materials. Chicago, IL: University of Chicago.

Bevington, P.R., and Robinson, D.K. (1992). Data reduction and error analysis for the physical sciences. Second edition. New York, NY: McGraw-Hill.

Beloin-Saint-Pierre, D., Heijungs, R., and Blanc, I. (2014). The ESPC (enhanced structural path analysis) method: a solution to an implementation challenge for dynamic life cycle assessment studies. International Journal of Life Cycle Assessment, 19(4), 861-871.

Björkland, A.E. (2002). Survey of approaches to improve reliability in LCA. International Journal of Life Cycle Assessment, 7(2), 64-72.

Bose, S., Kuila, T., Nguyen, T.X.H., Kim, N.H., Lau, K-t., and Lee, J.H. (2011). Polymer membranes for high temperature proton exchange membrane fuel cell: recent advances and challenges. Progress in Polymer Science, 36(6), 813-843.

Boustead, I. (2005a). Eco-profiles of the European plastics industry. Polyamide 6 (Nylon 6). Plastics Europe, Brussels.

Boustead, I. (2005b). Eco-profiles of the European plastics industry. Polyamide 66 (Nylon 66). Plastics Europe, Brussels.

Capello, C., Hellweg, S., and Hungerbühler, K. (2008). Environmental assessment of waste-solvent treatment options: Part II: General rules of thumb and specific recommendations. Journal of Industrial Ecology, 12(1), 111-127.

Cellura, M., Long, S., and Mistretta, M. (2011).Sensitivity analysis to quantify uncertainty in life cycle assessment: the case study of an Italian tile. Renewable and Sustainable Energy Review, 15(9), 4697-4705.

Chanaron, J-J. (2007). Life cycle assessment practices: benchmarking selected European automobile manufacturers. International Journal of Product Lifecycle Management, 2(3), 290-311.

Chandan, A., Hattenberger, M., El-kharouf, A., Du, S., Dhir, A., Self, V., Pollet, B.G., Ingram, A., Bujalski, W. (2013). High temperature (HT) polymer electrolyte membrane fuel cells (PEMFC) - a review. Journal of Power Sources, 231, 264-278.

Chen, X., and Corson, M.S. (2014). Influence of emission-factor uncertainty and farm-characteristic variability in LCA estimates of environmental impacts of French dairy farms. Journal of Cleaner Production, 81, 150157. 
Chester, M., and Horvath, A. (2008). Environmental life-cycle assessment of passenger transportation: a detailed methodology for energy, greenhouse gas, and criteria pollutant inventories of automobiles, buses, light rail, heavy rail and air. Berkeley, CA: University of California, Berkeley.

Chomkhamsri, K., Wolf, M-A., and Pant, R. (2011). International reference life cycle data system (ILCD) handbook: review schemes for life cycle assessment. In Finkbiener, M. (ed.), Towards life cycle sustainability management (pp. 107-117). Dordrecht, the Netherlands: Springer.

Ciambrone, D.F. (1997). Environmental life cycle analysis. Boca Raton, FL: CRC Press.

Collado-Ruiz, D., and Ostad-Ahmad-Ghorabi, H. (2010). Comparing LCA results out of competing products: developing reference ranges from a product family approach. Journal of Cleaner Production, 18(4), 355364.

Collet, P., Hélias, A., Lardon, L, and Steyer, J-P. (2011). In Finbeiner, M., (ed.), Towards life cycle sustainability management, pp. 119-130. Dordrecht, the Netherlands Springer Science + Business Media.

Cooper, J.S., and Fava, J.A. (2006). Life-cycle assessment practitioner survey: summary of results. Journal of IndustrialEcology, 10(4), 12-14.

Cooper, J., Fava, J., and Baer, S. (2008). Life cycle assessments of buildings in North America: demand and supply. Journal of Industrial Ecology, 12(1), 7-9.

Curran, M.A. (2007). Studying the effect on system preference by varying coproduct allocation in creating life-cycle inventory. Environmental Science \& Technology, 41(20), 7145-7151.

Dawkins, R. (1986). The blind watchmaker. New York, NY: W.W. Norton.

De Smet, B., and Stalmans, M. (1996). LCI data and data quality. International Journal of Life Cycle Assessment, l(2), 96-104.

Dhingra, R., Overly, J.G., and Davis, G.A. (1999). Life-cycle environmental evaluation of aluminum and composite intensive vehicles. Knoxville, TN: Center for Clean Products and Clean Technologies, University of Tennessee.

Dixit, M.K., Fernández-Solís, J.L., Lavy, S., and Culp, C.H. (2010). Identification of parameters for embodied energy measurement: a literature review. Energy and Buildings 42(8), 1238-1247.

Dresen, B., and Jandewerth, M. (2012). Integration of spatial analyses into LCA—calculating GHG emissions with geoinformation systems. International Journal of Life Cycle Assessment, 17(9), 1094-1103.

Dreyer, L.C., Hauschild, M.Z., and Schierbeck, J. (2006). A framework for social life cycle impact assessment. International Journal of Life Cycle Assessment, 11(2), 89-97.

Eckelman, M.J., Zimmerman, J.B., and Anastas, P.T. (2008). Toward green nana: E-factor analysis of several nanomaterial syntheses. Journal of Industrial Ecology, 12(3), 316-328.

Ekvall, T., and Finnveden, G. (2001). Allocation in ISO 14041 — a critical review. Journal of Cleaner Production, 9(3), 197-208.

Ekvall, T., Assefa, G., Björkland, A., Eriksson, O., Finnveden, G. (2007). What life-cycle assessment does and does not do in assessments of Waste Management. Waste Management, 27(8), 989-996.

Elcock, D. (2007). Life-cycle thinking for the oil and gas exploration and production industry. Oak Ridge, TN: U.S. Department of Energy. 
Escobar, N., Ribal, J., Clemente, G., and Sanjuán, N. (2014). Consequential LCA of two alternative systems for biodiesel consumption in Spain, considering uncertainty. Journal of Cleaner Production, 79, 61-73.

Fava, J., Baer, S., and Cooper, J. (2009). Increased demands for life cycle assessments in North America. Journal of Industrial Ecology, 13(4), 491-494.

Fet, A. M. (1998). ISO 14000 as a Strategic Tool for Shipping and Shipbuilding. Journal of Ship Production, 14(3), $155-163$.

Fiksel, J. (2010, May). Evaluating supply chain sustainability. Chemical Engineering Progress, 106(5), 28-36.

Finkbeiner, M., Inaba, A., Tan, R.B.H., Christiansen, K., and Llüppel, H-J. (2006). The new international standards for life cycle assessment: ISO 14040 and ISO 14044. International Journal of Life Cycle Assessment, $11(2), 80-85$.

Finkbeiner, M. (2009). Carbon footprinting-opportunities and threats. International Journal of Life Cycle Assessment, 14(2), 91-94.

Finkel, A. M. (1990). Confronting uncertainty in risk management-A guide for decision-makers. Washington, DC: Resources for the Future.

Field, F., Kirchain, R., and Clark, J. (2001). Life-cycle assessment and temporal distributions of emissions. Developing a fleet-based analysis. Journal of Industrial Ecology, 4(2), 71-91

Fleischer, G., Dose, J., and Hildenbrand, J. (2004). Use of generic data in LCA-studies. In Proceedings of the International Workshop on Quality of Life Cycle Inventory (LCI) Data, October 20-21, 2003, Karlsruhe, Germany (pp. 1-4). Karlsruhe, Germany: Forschungszentrum Karlsruhe.

Funtowicz, S.O., and Ravetz, J.R. (1990). Uncertainty and quality in science for policy. Dordrecht, the Netherlands: Kluwer Academic Publishers.

Gasafi, E., Meyer, L., and Schebek, L. (2003). Using life-cycle assessment in process design: supercritical waster gasification of organic feedstocks. Journal of Industrial Ecology, 7(3-4), 75-91.

Geyer, R., Stoms, D.M., Lindner, J.P., Davis, F.W., and Wittstock, B. (2010). Coupling GIS and LCA for biodiversity assessments of land use: part 1: inventory modeling. International Journal of Life Cycle Assessment, 15(7), 692-703.

Goedkoop, M., Hofstetter, P., Müller-Wenk, R., and Spriemsma, R. (1998). The Eco-Indicator 98 explained. International Journal of Life Cycle Assessment, 3(6), 352-360.

Goedkoop, M., Oele, M., Leijting, J., Ponsioen, T, and Meijer, E. (2013). Introduction to LCA with SimaPro. Amersfoort, the Netherlands: Pré Consultants bv.

Graedel, T.E. (1998). Streamlined life-cycle assessment. Upper Saddle River, NJ: Prentice Hall.

Grjotheim, K., Kvande, H., and Zhuxian, Q. (1995). Key improvements to Hall-Héroult since the end of World War II. JOM, 47(11), 32-35.

Hammond, G., and Jones, C. (2008). Inventory of carbon and energy (ICE), version 1.6a. Bath, UK: University of Bath.

Hawkins, T.R., Singh, B., Majeau-Bettez, and Strømman, A.H. (2013). Comparative environmental life cycle assessment of conventional and electric vehicles. Journal of Industrial Ecology, 17(1), 53-64. 
Heijungs, R. (1994). A generic method for the identification of options for cleaner products. Ecological Economics, $10(1), 69-81$.

Heijungs, R. (1996). Identification of key issues for further investigation in improving the reliability of life cycle assessments. Journal of Cleaner Production, 4(3-4), 159-166.

Heijungs, R., and Frischknecht, R. (2005). Representing statistical distributions for uncertain parameters in LCA. International Journal of Life Cycle Assessment, 10(4), 248-254.

Heijungs, R., and Huijbregts, M.A.J. (2004). A review of approaches to treat uncertainty in LCA. In Proceedings of the 2nd Biennial Meeting of iEMSs, Complexity and integrated resources management, 14-17 June 2004, Osnabrück, Germany (pp. 332-339). Manno, Switzerland: International Environmental Modelling and Software Society (iEMS)

Heijungs, R., Huppes, G., and Guinée. (2010). Life cycle assessment and sustainability analysis of products, materials and technologies. Towards a scientific framework for sustainable life cycle analysis. Polymer Degradation and Stability, 95(3), 422-428.

Heiskanen, E. (2000). Institutionalization of life-cycle thinking in the everyday discourse of market actors. Journal of Industrial Ecology, 4(4), 31-45.

Held, M., and Klöpffer, W. (2000). Life cycle assessment without time? Time matters in life cycle assessment. GAIA, 9(2), 101-108.

Helmes, R.J.K., Huijbregts, M.A.J., Henderson, A.D., and Jolliet, O. (2012). Spatially explicit fate factors of phosphorous emissions to freshwater at the global scale. International Journal of Life Cycle Assessment, 17(5), 646-654.

Hendrickson, C.T., Horvath, A., Joshi, S., Klausner, M., Lave, L.B., and McMichael, F.C. (1997). Comparing two life cycle assessment approaches: a process model vs. economic input-output-based assessment. In Proceedings of the 1997 IEEE International Symposium on Electronics and the Environment, San Francisco, CA, 5-7 May, 1997 (pp. 176-181). Washington, DC: Institute of Electrical and Electronics Engineers (IEEE).

Hendrickson, C.T., Lave, L.B., and Matthews, H.S. (2006). Environmental life cycle assessment of goods and services. An input-output approach. Washington, DC: Resources for the Future.

Hertwich, E.G., and Pease, W.S. (1998). ISO 14042 Restricts use and development of impact assessment. International Journal of Life Cycle Assessment, 3(4), 180-181.

Hertwich, E.G., McKone, T.E., and Pease, W.S. (2000). A systematic uncertainty analysis of an evaluative fate and exposure model. Risk Analysis, 20(4), 439-454.

Hofstetter, P. (1998). Perspectives in life cycle impact assessment. A structured approach to combine models of the technosphere, ecosphere and valuesphere. Dordrecht, the Netherlands, Kluwer Academic Publishers.

Hofmeister, S., and Kümmerer, K. (2009). Substance, substance-flow management, and time, part II: temporal impact assessment (TIA) for substance-flow management. Journal of Environmental Management, 90(3), 1377-1384.

Hongo, K., and Maezono, R. (2011). Quantum Monte Carlo simulations with RANLUX random number generator. Progress in Nuclear Science and Technology, 2, 51-55.

Hongo, K, Maezono, R., and Miura, K. (2010). Random number generators tested on quantum Monte Carlo simulations. Journal of Computational Chemistry, 31(11), 2186-2194. 
Huijbregts, M.A.J. (1998a). Application of uncertainty and variability in LCA. Part I: a general framework for the analysis of uncertainty and variability in life cycle assessment. International Journal of Life Cycle Assessment, 3(5), 273-280.

Huijbregts, M.A.J. (1998b). Application of uncertainty and variability in LCA. Part II: dealing with parameter uncertainty and uncertainty due to choices in life cycle assessment. International Journal of Life Cycle Assessment, 3(6), 343-351.

Huijbregts, M.A.J., Norris, G., Bretz, R., Ciroth, A., Maurice, B., von Bahr, B., Weidema, B., and de Beaufort, A.S.H. (2001). Framework for modelling data uncertainty in life cycle inventories. International Journal of Life Cycle Assessment, 6(3), 127-132.

Huijbregts, M.A.J., Gilijamse, W., Ragas, A.M.J., and Reijnders, L. (2003). Evaluating uncertainty in environmental life-cycle assessment. A case study comparing two insulation options for a Dutch onefamily dwelling. Environmental Science and Technology, 37(11), 2600-2608.

Huijbregts, M.A.J., Rombouts, L.J.A., Hellweg, S., Frischnecht, R., Hendriks, A.J., Meent, D. van de., Ragas, A.M.J., Reijnders, L., and Struijs, J. (2006). Is cumulative fossil energy demand a useful indicator for the environmental performance of products? Environmental Science \& Technology, 40(3), 641-648.

International Aluminum Institute. (2011). Historical IAI Statistics. London, UK: IAI. Accessed August 13, 2011.

International Organization for Standardization (ISO). (1997). ISO 14040: environmental management-life cycle assessment—principles and framework. Geneva, Switzerland: International Organization for Standardization.

International Organization for Standardization (ISO). (2006a). CAN/CSA-ISO14040:06 (ISO14040:2006). Environmental management—life cycle assessment—principles and framework. Mississauga, ON: Canadian Standards Association (CSA).

International Organization for Standardization (ISO). (2006b). ISO 14040: environmental management-life cycle assessment—requirements and guidelines. Geneva, Switzerland: International Organization for Standardization.

Jensen, A.A., Hoffman, L., Møller, B.T., Schmidt, A., Christiansen, K., Elkington, J., and van Dijk, F. (1997). Life Cycle Assessment (LCA): A guide to approaches, experiences and information sources. Copenhagen: European Environment Agency.

Kennedy, D.J., Montgomery, D.C., and Quay, B.H. (1996). Stochastic environmental life cycle assessment modeling. A probabilistic approach to incorporating variable input data quality. International Journal of Life Cycle Assessment, 1(4), 199-207.

Kennedy, D.J., Montgomery, D.C., Rollier, D.A., and Keats, J.B. (1997). Assessing input data uncertainty in life cycle assessment inventory models. International Journal of Life Cycle Assessment, 2(4), 229-239.

Keoleian, G.A., Kar, K., Manion, M.M., and Bulkley, J.W. (1997). Industrial ecology of the automobile: a life cycle perspective. Warrendale, PA: Society of Automotive Engineers (SAE).

Khasreen, M.M., Banfill, P.F.G., and Menzies, G.F. (2009). Life-cycle assessment and the environment impact of buildings: a review. Sustainability, 1(3), 647-701.

Kim, S., and Overcash, M. (2000). Allocation procedure in multi-output process: an illustration of ISO 14041. International Journal of Life Cycle Assessment, 5(4), 221-228.

Klöpffer, W. (1997). Peer (Expert) review in LCA according to SETAC and ISO 14040: theory and practice. International Journal of Life Cycle Assessment, 2(4), 183-184. 
Klöpffer, W. (2009). Experiences with the critical review process of aluminium LCI data. International Journal of Life Cycle Assessment, 14(Suppl. 1), S45-S51.

Krewitt, W., Trunkenmüller, A., Bachmann, T.M., and Heck, T. (2001). Country-specific damage factors for air pollutants: a step towards site dependent life cycle impact assessment. International Journal of Life Cycle Assessment, 6(4), 199-210.

Klüppel, H-J. (1998). ISO 14041: environmental management—life cycle assessment—goal and scope definitioninventory analysis. International Journal of Life Cycle Assessment, 3(6), 301.

Lecouls, H. (1999). ISO 14043: environmental management-life cycle assessment-life cycle interpretation. International Journal of Life Cycle Assessment, 4(5), 245.

Lee, S.G., and Xu, X. (2005). Design for the environment: life cycle assessment and sustainable package issues. International Journal of Environmental Technology and Management, 5(1), 14-41.

Leroy, Y., and Froelich, D. (2010). Qualitative and quantitative approaches dealing with uncertainty in life cycle assessment (LCA) of complex systems: towards a selective integration of uncertainty according to LCA objectives. International Journal of Design Engineering, 3(2), 151-171.

Levasseur, A., Lesage, P., margin, M., Deschenes, L., and Samson, R. (2010). Considering time in LCA: dynamic LCA and its application to global warming impact assessments. Environmental Science and Technology, 44(8), 3169-3174.

Levasseur, A., Lesage, P., Margni, M., and Samson, R. (2012). Biogenic carbon and temporary storage addressed with dynamic life cycle assessment. Journal of Industrial Ecology, 17(1), 117-128.

Li, S., Li, N., Li, J., and Goa, Y. (2012). Vehicle cycle energy and carbon dioxide analysis of passenger car in China. AASRI Procedia 2, 25-30.

Lo, S-C., Ma, H-W., and Lo, S-L. (2005). Quantifying and reducing uncertainty in life cycle assessment using the Bayesian Monte Carlo method. Science of the Total Environment, 340(1-3), 23-33.

Markowski, C.A., and Markowski, E.P. (1990). Conditions for the effectiveness of a preliminary test of variance. The American Statistician, 44(4), 322-326.

Marsmann, M. (1997). ISO 14040 - the first project. International Journal of Life Cycle Assessment, 2(3), 122-123.

Marsmann, M. (2000). The ISO 14040 family. International Journal of Life Cycle Assessment, 5(6), 317-318.

MathWorks .(2015). Kurtosis. Accessed 15/1/2015 from www.mathworks.com/help/stats/kurtosis.html

Matsumoto, M., and Nishimura, T. (1998). Mersenne twister: a 623-dimensionally equidistributed uniform psedorandom number generator. ACM Transactions on Modeling and Computer Simulation, 8(1), 3-30.

Maurice, B., Frischnecht, R., Coelho-Schwirtz, V., and Hungerbühler, K. (2000). Uncertainty analysis in life cycle inventory. Application to the production of electricity with French coal power plants. Journal of Cleaner Production, 8(2), 95-108.

McLean, H.L., and Lave, L.B. (1998). A life-cycle model of an automobile. Environmental Science \& Technology, 32(13), 322-330.

Memary, R., Giurco, D., Mudd, G., and Mason, L. (2012). Life cycle assessment: a time-series analysis of copper. Journal of Cleaner Production, 33, 97-108. 
Miller, S.A., and Theis, T.L. (2006). Comparison of life-cycle inventory databases. Journal of Industrial Ecology, 10(1-2), 133-147.

Mining, Minerals, and Sustainable Development Project. (2002). Breaking new ground: mining, minerals, and sustainable development : the report of the MMSD project, Volume 1. London, UK: International Institute for Environment and Development (IIED).

Moberg, Å., Borggren, C., Finnveden, G., and Tyskeng, S. (2010). Envrionmental impacts of electronic invoicing. Progress in Industrial Ecology—An International Journal, 7(2), 93-113.

Mongelli, I., Suh, S., and Huppes, G. (2005). A structure comparison of two approaches to LCA inventory data, based on the MIET and ETH databases. International Journal of Life Cycle Assessment, 10(5), 317-324.

Morgan, M.G., and Henrion, M. (1990). Uncertainty. A guide to dealing with uncertainty in quantitative risk and policy analysis. Cambridge, UK: Cambridge UniversityPress.

National Institute of Science and Technology. (2012). Engineering statistics handbook. National Institute of Standards and Technology, U.S. Department of Commerce: Gaithersburg, MD.

Newell, J.P., and Vos, R.O. (2001). "Papering” over space and place: product carbon footprint modeling in the global paper industry. Association of American Geographers, 101(4), 730-741.

Nigge, K-M. (2001). Generic spatial classes for human health impacts, part 1: methodology. International Journal of Life Cycle Assessment, 6(5), 257-264.

Norris, G.A. (2001). Integrating life cycle cost analysis and LCA. International Journal of Life Cycle Assessment, $6(2), 118-120$.

Ny, H., MacDonald, J.P., Broman, G., Yamamoto, R., and Robèrt, K-H. (2006). Sustainability constraints as system boundaries: an approach to making life-cycle management strategic. Journal of Industrial Ecology, 10(1-2), 61-77.

Ogilvie, J.F. (1984). A Monte-Carlo approach to error propagation. Computers and Chemistry, 8(3), 205-207.

Owens, J.W. (1997). Life-cycle assessment in relation to risk assessment: an evolving perspective. Risk Analysis, 17(3), 359-365.

Parker, R.P.M., Harrison, G.P., and Chick, J.P. (2007). Energy and carbon audit of an offshore wave energy converter. Proceedings of the Institution of Mechanical Engineers Part C: Journal of Power and Energy, 221(8), 1119-1130.

Peereboom, E.C., Kleijn, R., Lemkowitz, S., and Lundie, S. (1999). Influence of inventory data sets on life-cycle assessment results: a case study on PVC. Journal of Industrial Ecology, 2(3), 109-130.

Peighambardoust, S.J., Rowshanzamir, S., Amajadi, M. (2010). Review of the proton exchange membranes for fuel cell applications. International Journal of Hydrogen Energy, 35(17), 9349-9384.

Pelletier, N.L., Ayer, N.W., Tyedmers, P.H., Kruse, S.A., Flysjo, S.A., Robillard, G., Ziegler, F., Scholz, A.J., Sonesson, U. (2007). Impact categories for life cycle assessment research of seafood production systems: review and prospectus. International Journal of Life Cycle Assessment, 12(6), 414-421.

Pfister, S., Koehler, A., and Hellweg, S. (2009). Assessment the environmental impacts of freshwater consumption in LCA. Environmental Science and Technology, 43(11), 4098-4104. 
Phungrassami, H. (2008). A review of time consideration in life cycle assessment. Global Journal of Environmental Research, 2(2), 62-65.

Pinsonnault, A., Lesage, P., Levasseur, A., and Samson. (2014). Temporal differentiation of background systems in LCA: relevance of adding temporal information in LCI databases. International Journal of Life Cycle Assessment, 19(11), 1843-1853.

Pohl, C., Roš, M., Waldeck, B., and Dinkel, F. (1996). In Schaltegger, S. (ed.), Life cycle assessment (LCA)—quo vadis? (pp. 51-68). Basel, Switzerland: Birkhäuser Verlag.

Potting, J., and Hauschild, M.Z. (2006). Spatial differentiation in life cycle assessment: a decade of method development to increase the environmental realism of LCIA. International Journal of Life Cycle Assessment, 11(1 Supplement), 11-13.

Raynolds, M., Checkel, M.D., and Fraser,R.A. (1999). Application of Monte Carlo analysis to life cycle assessment. SAE Technical Paper 1999-10-011.

Reap, J., Bras, B., Newcomb, P.J., and Carmichael, C. (2003). Improving life cycle assessment by including spatial, dynamic and place-based modeling. In Proceedings of DETC '03 ASME 2003 Design Engineering Technical Conferences and Computers and Information in Engineering Conference, Chicago, Il, 2-6 September 2-6, 2003 (pp. 1-7). New York, NY: American Society of Mechanical Engineers (ASME).

Reap, J., Roman, F., Duncan, S., and Bras, B. (2008a). A survey of unresolved problems in life cycle assessment. Part 1: goal and scope inventory analysis. International Journal of Life Cycle Assessment, 13(4), 290-300.

Reap, J., Roman, F., Duncan, S., and Bras, B. (2008b). A survey of unresolved problems in life cycle assessment. Part 2: impact assessment and interpretation. International Journal of Life Cycle Assessment, 13(5), 374-388.

Reed, G.F., Lynn, F., and Meade, B.R. (2002). Use of coefficient of variation in assessing variability of quantitative assays. Clinical and Diagnostic Laboratory Immunology, 9(6), 1235-1239.

Reich, M.C. (2005). Economic assessment of municipal waste management systems - case studies using a combination of life cycle assessment (LCA) and life cycle costing (LCC). Journal of Cleaner Production, 13(3), 253-263.

Renó, M.L.G., Lora, E.E.S., Palacio, J.C.E., Venturini, O.J., Buchgeister, J., and Almazan, O. (2011). A LCA (life cycle assessment) of the methanol production from sugarcane bagasse. Energy, 36(6), 3716-3726.

Roy, P-O., Deschênes, L., and Margni, M. (2013). Uncertainty and spatial variability in characterization factors for aquatic acidification at the global scale. International Journal of Life Cycle Assessment, 19(4), 882-890.

Ryding, S-O. (1999). ISO 14042: environmental management—life cycle assessment—life cycle impact assessment. International Journal of Life Cycle Assessment, 4(6), 307.

Saad, R., Margni, M., Koellner, T., Wittstock, B., and Deschênes., L. (2011). Assessment of land use impacts on soil ecological functions: development of spatially differentiated characterization factors within a Canadian context. Int J Life Cycle Assess, 16(3), 198-211.

Sablayrolles, C., Gabrielle, B., and Montrejaud-Vignoles, M. (2010). Life cycle assessment of biosolids land application and evaluation of the factors impacting human toxicity through plant uptake. Journal of Industrial Ecology, 14(2), 231-241. 
Sakai, S-I, Yoshida, H., Hiratsuka, J., Vandecasteele, C., Kohlmeyer, R., Rotter, V.S., ... Yano, J. (2014). An international comparative study of end-of-life vehicle (ELV) recycling systems. Journal of Material Cycles and Waste Management, 16(1), 1-20.

Salmone, R., Mondello, F., Lanuzza, F., and Micali, G. (2005). Environmental assessment: an eco-balance of a recycling plant for spent lead-acid batteries. Environmental Management, 35(2), 206-219.

Säynäjoki, A., Heinonen, J., and Junnila, S. (2012). A scenario analysis of the life cycle greenhouse gas emissions of a new residential area. Environmental Research Letters, 7(3), 1-10.

Santero, N.J., Masanet, E., and Horvath, A. (2011a). Life-cycle assessment of pavements. Part I: critical review. Resources, Conservation and Recycling, 55(9-10), 801-809.

Santero, N.J., Masanet, E., and Horvath, A. (2011b). Life-cycle assessment of pavements. Part II: filling the research gaps. Resources, Conservation and Recycling, 55(9-10), 810-818.

Schaltegger, S. (1996). Eco-efficiency of LCA. The necessity of a site-specific approach. In Schaltegger, S. (ed.), Life cycle assessment (LCA)—quo vadis? (pp. 133-149). Basel, Switzerland: Birkhäuser Verlag.

Schau, E.M., and Fet, A.M. (2008). LCA studies of food products as background for environmental product declarations. International Journal of Life Cycle Assessment, 13(3), 255-264.

Schexnayder, S.M., Das, S., Dhingra, R., Overly, J.G., Tonn, B.E., Peretz, J.H., Waidley, G., and Davis, G.A. (2001). Environmental evaluation of new generation vehicles and vehicle components. Washington, DC: U.S. Department of Energy.

Schmitz, S., and Paulini, I. (1999). Valuation as an element of life cycle assessments: German Federal Environmetnal Agency method for impact indicator standardization, impact category grouping (ranking), and interpretation in accordance with ISO 14042 and 14043. Dessau, Germany: Federal Environmental Agency.

Schuckert, M. (1996). Life cycle analysis: getting the total picture on vehicle engineering alternatives. Automotive Engineering, 104(3), 49-52.

Schweimer, G. W., and M. Levin. (2000). Life cycle inventory for the Golf A4. Wolfsburg, Germany: Volkswagen AG.

Scientific Applications International Corporation. (2006). Life cycle assessment: principles and practice. Cincinnati, OH: U.S. Environmental Protection Agency.

Sheate, W.R. (2010). Tools, techniques and approaches for sustainability: collected writings in environmental assessment policy and management. Singapore: World Scientific.

Silverman, B.W. (1998). Density estimation for statistics and data analysis. Boca Raton, FL: CRC Press.

Sørensen, J.B. (2002). The use and misuse of the coefficient of variation in organizational demography research. Sociological Methods and Research, 30(4), 475-491.

Stasinopoulos, P., Compston, P., Newell, B., and Jones, H.M. (2012). A system dynamics approach in LCA to account for temporal effects - a consequential energy LCI of car body-in-whites. International Journal of Life Cycle Assessment, 17(2), 199-207.

Staudinger, J., and Keoleian, G.A. (2001). Management of end of life vehicles (ELVs) in the US. Ann Arbor, MI: University of Michigan. 
Steen, B. (1997). On uncertainty and sensitivity of LCA-based priority setting. Journal of Cleaner Production, 5(4), 255-262.

Steinberger, J.K., Friot, D., Jolliet, O., and Erkman, S. (2009). A spatially explicit life cycle inventory of the global textile chain. International Journal of Life Cycle Assessment, 14(5), 433-455.

Stodolsky, F., Vyas, A., Cuenca, R., and Gaines, L. (1995). Life-cycle energy savings from aluminum intensive vehicles. Paper presented at SAE 1995 Total Cycle Conference and Exposition, Vienna, Austria, October 16-19, 1995.

Suh,S., Leighton, M., Tomar, S., and Chen, C. (2013). Interoperability between ecoinvent ver. 3 and US LCI database: a case study. International Journal of Life Cycle Assessment, online 11 June 2013.

Sullivan, J., and Hu, J. (1995). Life cycle energy analysis for automobiles. SAE Technical Paper 951829. Warrendale, PA: Society of Automotive Engineers (SAE).

Sullivan, J.L., Burnham, A., and Wang, M.Q. (2012). Model for the part manufacturing and vehicle assembly component of the vehicle life cycle inventory. Journal of Industrial Ecology, 17(1), 143-153.

Svanes, E., Vold, M., and Hanssen, O.J. (2011). Effect of different allocation methods on LCA results of products from wild-caught fish and on the use of such results. International Journal of Life Cycle Assessment, 16(6), 512-521.

Takano, A., Winter, S., Hughes, M., and Linkosalmi, L. (2014). Comparison of life cycle assessment databases: a case study on building assessment. Building and Environment, 79, 20-30.

Tan, R.R., Culaba, A.B., and Purvis, M.R.I. (2002). Application of possibility theory in the life-cycle inventory assessment of biofuels. International Journal of Energy Research, 26(8), 737-745.

Tsoutsos, T., Kouloumpis, V., Zafiris, T., and Foteinis, S. (2010). Life cycle assessment for biodiesel production under Greek climate conditions. Journal of Cleaner Production, 18(4), 328-335.

Udo de Haes, H.A. (2006). How to approach land use in LCIA or, how to avoid the Cinderella effect? Comments on 'Key Elements in a Framework for Land Use Impact Assessment Within LCA'. International Journal of Life Cycle Assessment, 11(4), 219-221.

Udo de Haes, H.A., and Jolliet, O. (1999). How does ISO/DIS 14042 on life cycle impact assessment accommodate current best available practice? International Journal of Life Cycle Assessment, 4(2), 75-80.

Udo de Haes, H.A., Finnveden, G., and Goedkoop, M. (2002). Life-cycle impact assessment: striving towards best practice. Pensacola, FL: SETAC Press.

Udo de Haes, H.A., Heijungs, R., Suh, S., and Huppes, G. (2004). Three strategies to overcome the limitations of life-cycle assessment. Journal of Industrial Ecology, 8(3), 19-32.

United Nations Environmental Programme (UNEP). (2005). Life cycle approaches: the road from analysis to practice. Paris, France: UNEP.

Upham, P., Dendler, L., and Bleda, M. (2011). Carbon labelling of grocery products: public perceptions and potential emissions reductions. Journal of Cleaner Production, 19(4), 348-355.

U.S. Department of Energy. (2012a). Buildings energy data book. Notes on embodied energy. http://buildingsdatabook.eren.doe.gov/TableView.aspx?table=Notes

U.S. Department of Energy. (2012b). Energy intensity indicators: efficiency vs. intensity. www1.eere.energy.gov/analysis/eii_efficiency_intensity.html 
U.S. Department of Energy. (2013). Multi-year research, development and demonstration plan. Planned program activities for 2011-2010. U.S. Department of Energy, Fuel Cell Technologies Office, Washington.

U.S. Environmental Protection Agency. (1989). Exposure factors handbook. Washington, DC: U.S. Environmental Protection Agency.

U.S. Environmental Protection Agency. (1997). Guiding principles for Monte Carlo analysis. Washington, DC: U.S. Environmental Protection Agency.

Usva, K., Saarinen, M., Katajajuuri, J-M., and Kurppa, S. (2009). Supply chain integrated LCA approach to assess environmental impacts of food production in Finland. Agriculture and Food Science, 18(3-4), 460-476.

Van den Berg, N.W., Huppes, G., Lindeijer, E.W., Van der Ven, B.L., and Wrisberg, M.N. (1999). Quality assessment for LCA. Lieden, the Netherlands: Lieden University.

Wang, M. (2012). GREET1 Model [Computer software]. Argonne, IL: Argonne National Laboratory.

Wang, E., and Shen, Z. (2013). A hybrid Data Quality Indicator and statistical method for improving uncertainty analysis in LCA of complex system - application to the whole-building embodied energy analysis. Journal of Cleaner Production, 43, 166-173.

Weaver, W. (1948). Science and complexity. American Scientist, 36(4), 536-544.

Weidema, B.P. (1998). Multi-user test of the data quality matrix for product life cycle inventory data. International Journal of Life Cycle Assessment, 3(5), 259-265.

Weidema, B.P., and Wesnaes, M.S. (1996). Data quality management in life cycle inventories — an example of using data quality indicators. Journal of Cleaner Production, 4(3-4), 167-174.

Weidema, B.P., Thrane, M., Christensen, P., Schmidt, J., and Løkke, S. (2008). Carbon footprint. A catalyst for life cycle assessment. Journal of Industrial Ecology, 12(1), 3-6.

Weiss, D.J. (2005). Analysis of variance and functional measurement: a practical guide. Oxford, UK: Oxford University Press.

Whitehead, N.P., Scherer, W.T., Louis, G.L., and Smith, M.C. (2010). Improving lifecycle assessment of biofuel systems. In Green Technologies Conference, 2010 IEEE, April 15-16, 2010, Grapevine, TX (pp. 1-9). Washington, DC: Institute of Electrical and Electronics Engineers (IEEE).

Worrell, E., de Beer, J., Blok, K. (1993). Energy conservation in the iron and steel industry. In Pilavachi, P.A. (Ed.), Energy efficiency in process technology (pp. 91-100). Elsevier Applied Science, London.

Yuan, C., Simon, R., Mady, N., and Dornfeld, D. (2009). Embedded temporal difference in life cycle assessment: case study on VW Golf A4 car. In Proceedings of IEEE International Symposium on Systems and Technology, May 18-20, 2009, Phoenix, AZ (pp. 1-6). Washington, DC: Institute of Electrical and Electronics Engineers (IEEE).

Zamel, N., and Li, X. (2006). Life cycle analysis of vehicles powered by a fuel cell and by internal combustion engine in Canada. Journal of Power Sources, 155(2), 297-310. 